University of Louisville

ThinkIR: The University of Louisville's Institutional Repository

\title{
Characterization of Filifactor alocis and its immune evasion strategies employed against human neutrophils.
}

Cortney Linn Armstrong

University of Louisville

Follow this and additional works at: https://ir.library.louisville.edu/etd

Part of the Immunology of Infectious Disease Commons

\section{Recommended Citation}

Armstrong, Cortney Linn, "Characterization of Filifactor alocis and its immune evasion strategies employed against human neutrophils." (2017). Electronic Theses and Dissertations. Paper 2769. https://doi.org/10.18297/etd/2769

This Doctoral Dissertation is brought to you for free and open access by ThinkIR: The University of Louisville's Institutional Repository. It has been accepted for inclusion in Electronic Theses and Dissertations by an authorized administrator of ThinkIR: The University of Louisville's Institutional Repository. This title appears here courtesy of the author, who has retained all other copyrights. For more information, please contact thinkir@louisville.edu. 


\title{
CHARACTERIZATION OF FILIFACTOR ALOCIS AND ITS IMMUNE EVASION STRATEGIES EMPLOYED AGAINST HUMAN NEUTROPHILS
}

\author{
By: \\ Cortney Linn Armstrong \\ B.S., Florida State University, 2012 \\ M.S., University of Louisville, 2014

\begin{abstract}
A Dissertation
Submitted to the Faculty of the

School of Medicine of the University of Louisville

in Partial Fulfillment of the Requirements for the Degree of
\end{abstract}

Doctor of Philosophy in Microbiology and Immunology

Department of Microbiology and Immunology

University of Louisville School of Medicine

Louisville, KY

August 2017 
Copyright 2017 by Cortney Linn Armstrong

All rights reserved 



\title{
CHARACTERIZATION OF FILIFACTOR ALOCIS AND ITS IMMUNE EVASION STRATEGIES EMPLOYED AGAINST HUMAN NEUTROPHILS
}

\author{
By: \\ Cortney Linn Armstrong \\ B.S., Florida State University, 2012 \\ M.S., University of Louisville, 2014 \\ A Dissertation Approved on
}

August 4, 2017

by the following Dissertation Committee:

Dissertation Director

Silvia M. Uriarte

Pascale Alard (Co-Mentor)

Richard J. Lamont

Richard D. Miller

James E. Graham 


\title{
DEDICATION
}

\author{
This dissertation is dedicated to \\ my parents Tim and Lisa Armstrong \\ and to my fiancé Jared Mushill
}

who have provided me with unconditional love and support

throughout my journey to obtaining my Ph.D. 


\section{ACKNOWLEDGEMENTS}

First, I would like to thank my mentor, Dr. Silvia Uriarte. From day one in my rotation, she has made me feel welcome and like an important part of the lab. I want to thank her for taking the chance on me, as her first Ph.D. student. She has been a wonderful mentor and friend along my graduate school career, always taking time to answer any questions, no matter how big or small. I have developed as a person and as a scientist, as she always pushed me to work to my fullest potential. I sincerely thank her for all she has done for me and wish her and her lab all the best in their future endeavors.

Next, I would like to thank my dissertation committee, Dr. Richard Lamont, Dr. Pascale Alard, Dr. Richard Miller and Dr. Jim Graham. You all have been very supportive of me and always taken time to make sure our meetings were productive and helpful. I appreciate your enthusiasm in my projects and all your guidance and help along the way. I would especially like to thank Dr. Lamont for his role as my co-mentor. Thank you for providing a second lab family and a place to grow my bacteria. You have always had your door open to me and treated me as one of your own lab members and I greatly appreciate your time and support.

To the Uriarte lab, thank you for being my second family! To Junyi Le, my first mentor in the lab when I joined, thank you for your patience in teaching me how to work with neutrophils and F. alocis. I would also like to thank Terri Manning for her expertise in neutrophil isolation, our lab work would not be possible without her. I would like to thank Shifu Tian, for his

friendship and mentorship in lab life as well as outside the lab. I have enjoyed our many chats 
laugh, lunch times, tea breaks, Chinese lessons and dance breaks. Thanks to Dr. Aruna Vashishta for being my lab mom. You have been so supportive of me and have been willing to answer any question, science-related or otherwise. Thanks for always staying late with me, so we can both arrive safely at our cars. A special thanks to my wonderful friend and lab mate, Dr. Shuvasree SenGupta, who has always been there for me as we worked towards the final finish line! Thank you for your talks through scientific puzzles as well as your friendship along the way. I look up to you as a big sister and a great scientist. I would like to thank Jacob Edmisson for his dedication to our lab work and his help on my project during his undergraduate studies. Thank you for bringing your great ideas and also for your friendship, I miss having you in the lab. I would like to thank Chris Klaes, as he has been a great asset to our lab and willing to help take on the project once I leave the lab. Thanks for your help in troubleshooting and also for your friendship. To Dr. Qian (Tiana) Wang, the Filifactor alocis queen, I would like to thank you for your patience and time in training me on how to culture and maintain the bacteria, for always answering any question I had and for your friendship. Thank you to the past and present lab members, Adam, Lisandra, Tutonda, Emeri, Irina, Hazel, Juili, and Thomas, that I have worked with during my time in the lab, it takes all of us working together to be successful and I appreciate all you have done for me as my lab family.

Lastly, I would like to thank my family and friends. Without their constant love and support, none of this would have been possible. I cannot emphasize enough how much I appreciate them all! To my parents, Tim and Lisa, who have always been there for me and have pushed me to follow my dreams, even if it meant moving to Louisville. Thank you for providing encouragement and for always believing in me. To my brother, Chad, thank you for supporting me and always being interested in my work, asking questions to try to understand what it is that I do each day. For our weekly chats, I appreciate them, you are a wonderful guy. To my fiancée, Jared, who deserves more than a few sentences in a dissertation acknowledgement. Thank you for 
being the person who day in and day out, through the good days and the bad, always believed in me, encouraged me and never cared about how many late nights I spent in the lab or at my computer. For listening to me vent, for cooking me dinner and cleaning the apartment, for your patience with me for all my computer needs. I love you and appreciate you so very much.

The Beatles say it best, "I get by with a little help from my friends." To my best friends in Florida, Christine Murphy and Melanie Singer. Thank you both for your constant support, your visits, your comic relief and for always telling me I can do it! To my first friend in Louisville, Dr. Justin Hutcherson, thank you for your convincing me to come to Louisville, for your friendship and for always being there for me when I have a question about anything M \& I related. To Dr. Michael Connor, who has helped me so much along my graduate school career. Thank you for your help with live cell imaging, with ImageJ analysis, and for helping to format my dissertation. Also, thank you for being a great friend to Jared and I. To Dr. Dan Miller, thank you for expertise on all things oral microbiology and for your friendship. Thank you for introducing me to your wife, Katie Miller, who is a great friend. Thanks to both of you for being great friends and for providing a place to vent, a puppy friend for Luna, the beer and the laughs! 


\title{
ABSTRACT \\ CHARACTERIZATION OF FILIFACTOR ALOCIS AND ITS IMMUNE EVASION STRATEGIES EMPLOYED AGAINST HUMAN NEUTROPHILS
}

\author{
Cortney L. Armstrong \\ August 4, 2017
}

Periodontal disease is among the most common of inflammatory conditions and is caused by bacterial and host derived factors. The presence of bacteria drives the recruitment of neutrophils, professional phagocytes, to migrate to specific oral sites where they produce potent antimicrobials to kill their target. However, this inflammation and production of antimicrobials must be strictly regulated to minimize collateral host tissue damage. Human neutrophils recognized the oral pathogen Filifactor alocis through Toll-like receptor (TLR) 2 and upon binding, activated both p38 MAPK and ERK signaling pathways, known to be involved in neutrophil cell migration and degranulation. F. alocis also stimulated secretory vesicle and specific granule exocytosis and enhanced chemokinetic and chemotactic migration to interleukin (IL)-8, a key chemoattractant found in the oral cavity (Chapter 2).

Once these challenged neutrophils have arrived at their targeted site, they will employ oxidative-mediated killing mechanisms, operating intracellularly in the bacterial-containing phagosome, and extracellularly, in the extracellular space. Neutrophils effectively perform phagocytosis to internalize $F$. alocis into their phagosomal compartment, however minimal intracellular respiratory burst response is produced. In addition, $F$. alocis-challenged neutrophils produced minimal superoxide release, however the bacterial challenge primed neutrophils for an enhanced respiratory burst response. F. alocis survived neutrophil oxygen-dependent intracellular and extracellular killing mechanisms up to $4 \mathrm{~h}$ post-infection (Chapter 3). Activated neutrophils can also undergo neutrophil extracellular trap (NET) formation as a means to trap and potentially 
kill targets. NETs have been described to be formed in the oral cavity in response to oral biofilms. F. alocis failed to induce NETs from neutrophils, which may indicate this bacterium is unique to the oral cavity, as other oral bacteria Streptococcus gordonii and Peptoanaerobacter stomatis induce NETs. However, F. alocis can manipulate neutrophils and reduce their NET formation capacities to known pharmacological (PMA) and bacterial (S. gordonii) inducers (Chapter 4).

Overall, our results are the first to show how $F$. alocis effectively evades human neutrophil killing mechanisms and manipulates some of their functional responses. These results provide information about the pathogenic potential of $F$. alocis which would help delineate the role of this emerging pathogen in the development of periodontal disease. 


\section{TABLE OF CONTENTS}

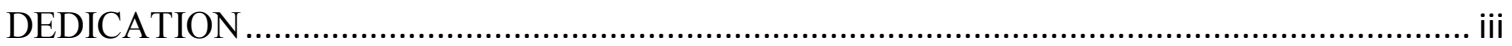

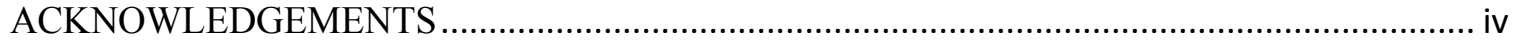

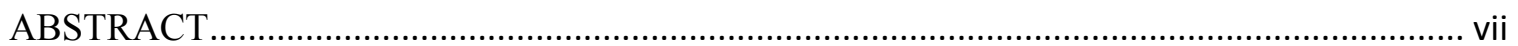

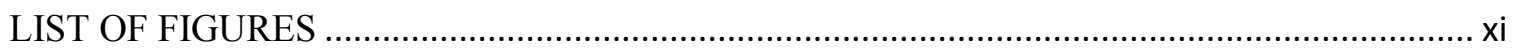

LIST OF TABLES........................................................................................................iii

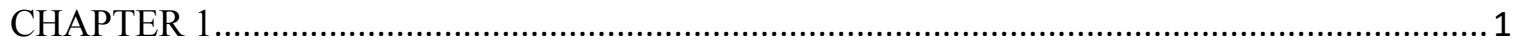

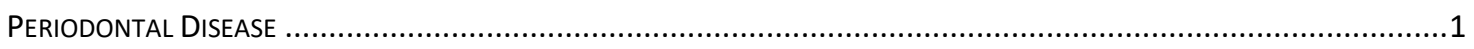

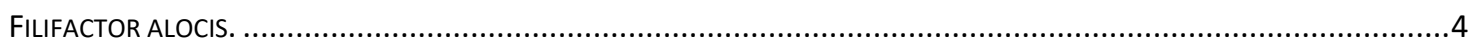

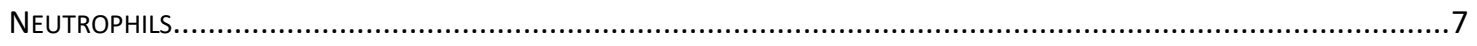

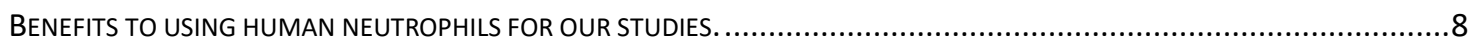

NEUTROPHIL MIGRATION- FROM THE BLOOD STREAM TO THE SITE OF INJURY, INFECTION, OR INFLAMMATION..................9

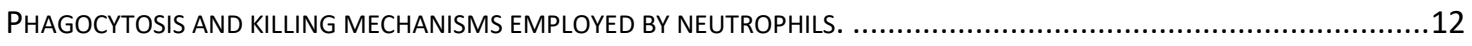

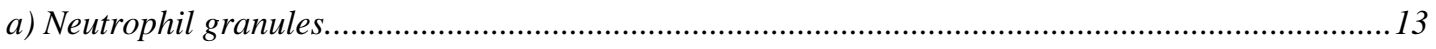

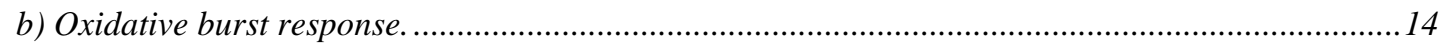

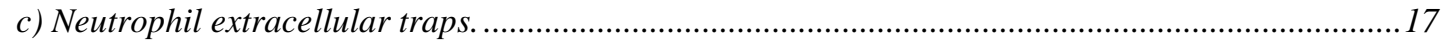

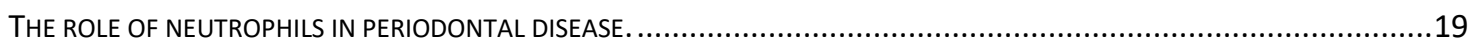

NEUTROPHIL SPECIFIC DISORDERS PLAY A ROLE IN PERIODONTAL DISEASE DEVELOPMENT ........................................21

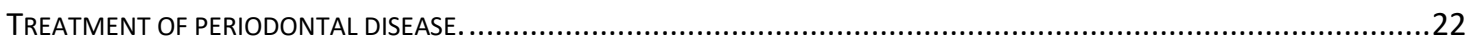

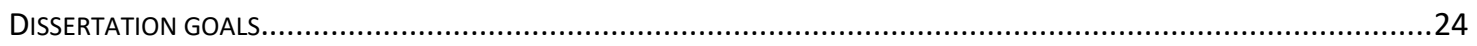

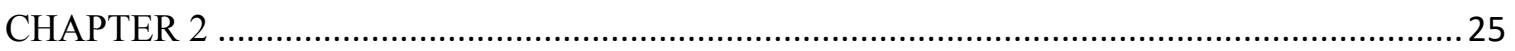

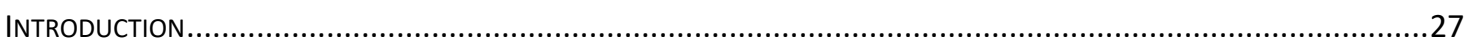

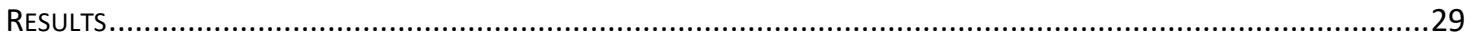

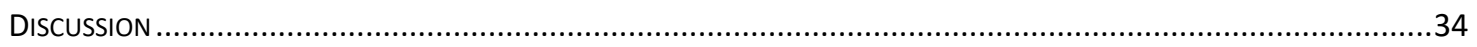

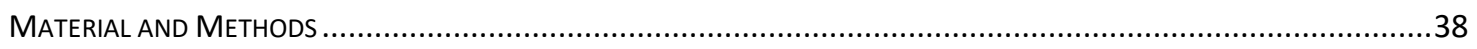

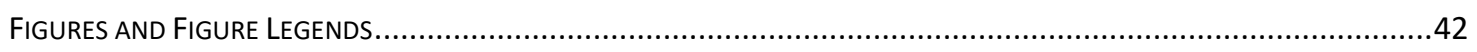

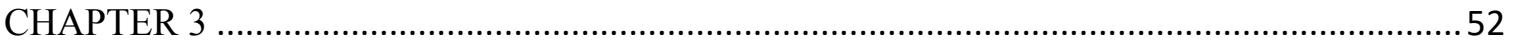

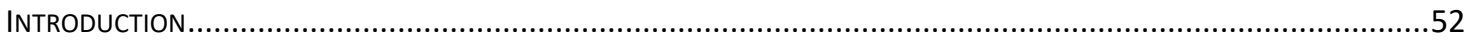

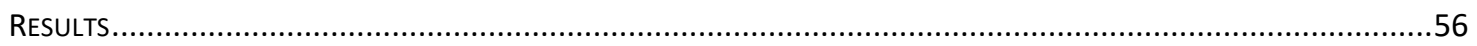

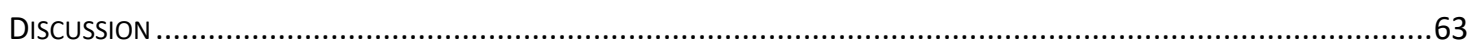

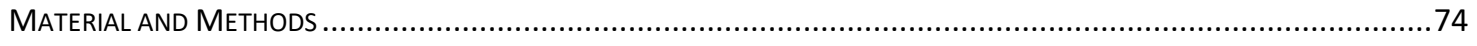

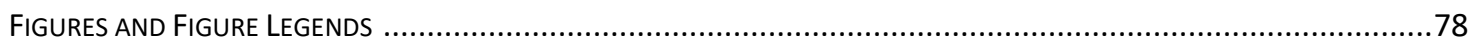




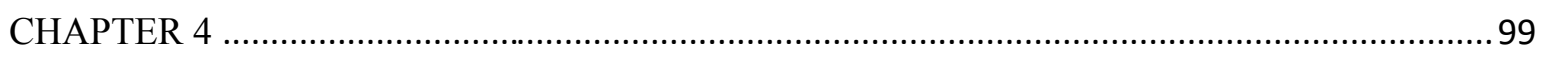

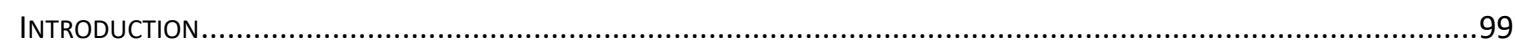

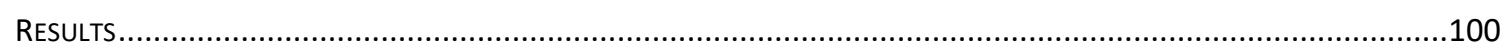

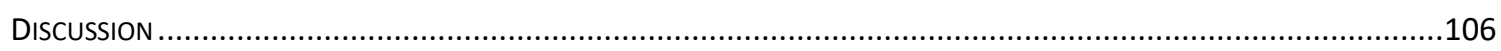

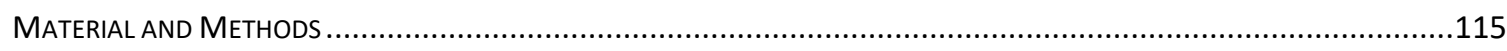

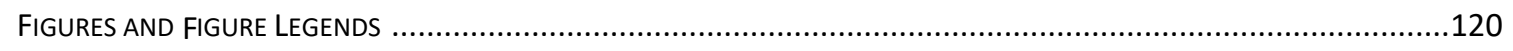

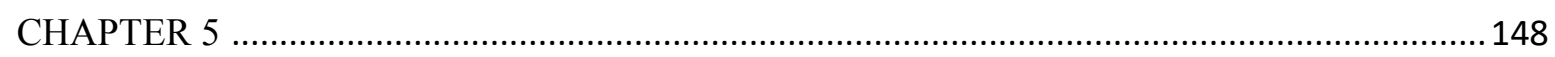

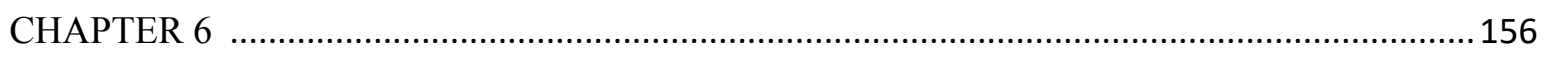

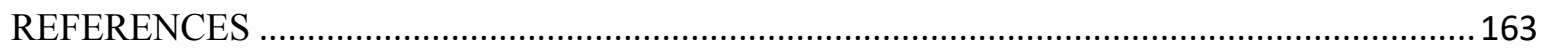

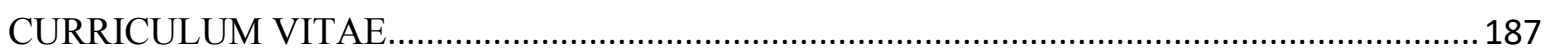




\section{LIST OF FIGURES}

Figure 1-1. Neutrophil granules and their hierarchal exocytosis........................................................14

Figure 1-2. NADPH oxidase activation and assembly leading to the respiratory burst response .................15

Figure 2-1. Effect of $F$. alocis stimulation on neutrophil chemotaxis. ................................................42

Figure 2-2. F. alocis-induced ERK1/2 and p38 MAPK activation in human neutrophils..........................43

Figure 2-3. TLR2 activation is involved in F. alocis-induced phosphorylation of both ERK1/2 and p38

MAPK.

Figure 2-4. F. alocis stimulation of secretory vesicle and specific granule exocytosis is p38 MAPK

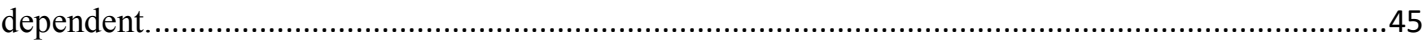

Figure 2-5. F. alocis challenge does not induce azurophil granule exocytosis. ........................................46

Figure 2-6. F. alocis interaction with TLR2 triggered secretory vesicle exocytosis. ................................47

Figure 2-7. Blocking neutrophil granule exocytosis inhibits $F$. alocis-induced random and directed migration

Figure 2-8. Schematic representation of $F$. alocis-induced neutrophil granule exocytosis, random and directed migration.

Figure 3-1. F. alocis remains viable intracellularly and extracellularly up to 4 h post-neutrophil challenge.

Figure 3-2. Neutrophils challenged with $F$. alocis produce minimal extracellular ROS production, but can prime neutrophils to secondary stimuli.

Figure 3-3. Neutrophils challenged with heat-killed $F$. alocis and F. alocis spent supernatant produce minimal extracellular ROS production, but can prime neutrophils to secondary stimuli.

Figure 3-4. F. alocis challenge of human neutrophils may signal through TLR $2 / 6$ .84

Figure 3-5. No difference in recruitment of gp91 ${ }^{\text {phox }}$ to bacteria-containing phagosomes between live and heat-killed $F$. alocis.

Figure 3-6. No difference in recruitment of p22 phox to bacteria-containing phagosomes between live and heat-killed $F$. alocis.

Figure 3-7. No difference in recruitment of $\mathrm{p} 47^{\text {phox }}$ to bacteria-containing phagosomes between live and heat-killed $F$. alocis at 15-30 mins.

Figure 3-8. No difference in recruitment of p67 phox to bacteria-containing phagosomes between live and heat-killed $F$. alocis.

Figure 3-9. Impaired recruitment of $\mathrm{p} 40^{\text {phox }}$ to viable $F$. alocis-containing phagosomes at 15 and 60 mins.95 Figure 3-10. Rac activation by neutrophils challenged with $F$. alocis is impaired in viable bacteria at 30 mins.

Figure 4-1. Opsonized $F$. alocis challenge fails to induce NET formation by human neutrophils.............121

Figure 4-2. Non-opsonized $F$. alocis challenge fails to induce NET formation by human neutrophils. .....123

Figure 4-3. Heat-killed $F$. alocis challenge fails to induce NET formation by human neutrophils............126

Figure 4-4. F. alocis fails to induce the release of extracellular neutrophil elastase. ..............................129

Figure 4-5. TNF- $\alpha$ pre-treatment fails to stimulate NET formation in response to challenge with $F$. alocis.

Figure 4-6. S. gordonii and P. stomatis induce significant NET formation in an MOI-dependent manner.134 Figure 4-7. Coinfection of $F$. alocis with $S$. gordonii or $P$. stomatis does not reduce or exacerbate NET

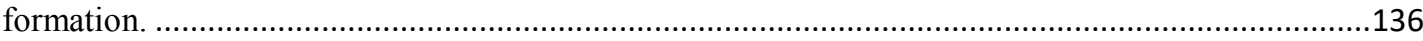

Figure 4-8. F. alocis fails to degrade NETs formed by $S$. gordonii and $P$. stomatis..............................138 Figure 4-9. F. alocis pre-treatment can cause significant decrease in NET formation induced by PMA....141 
Figure 4-10. F. alocis pre-treatment significantly decreases NET formation induced by S. gordonii but not

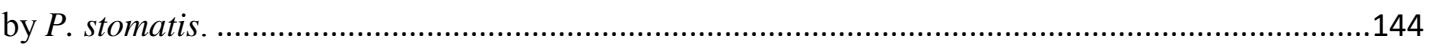

Figure 4-11. S. gordonii and P. stomatis induce NET formation in an MOI- and ROS-dependent manner.

Figure 6-1. The effects of $F$. alocis challenge on human neutrophil effector mechanisms. .147 162 


\section{LIST OF TABLES}

Table 1. NADPH oxidase component assembly and activation impairment upon challenge ........................69 


\section{CHAPTER 1 \\ INTRODUCTION}

\section{Periodontal Disease}

Periodontitis is defined as an infection-induced chronic inflammatory disease impacting the tissues supporting the teeth $(1,2)$. Periodontal tissues are easily accessible to both bacteria

and immune host cells, as it is comprised of a porous membrane of gingival epithelia cells with no protective mucous layer, as observed in the intestinal epithelium $(1,3,4)$. The induction of infectious diseases requires both microbial and immunological factors, which are the shift in microbiota content and the destructive inflammatory response, respectively (5). The increase in the number of host inflammatory cells and mediators leads to resorption of the alveolar bone, a key marker of periodontitis $(1,6-8)$.

Periodontal disease affects over 49 million people in the United States alone $(2,9-11)$ and it has been observed that $\sim 50 \%$ of the population 30 years and older has experienced some form of the disease $(10,12)$. Disease prevalence is increased with age and more common in males than in females, which may be due to poorer oral hygiene practices, higher usage of tobacco products, and less frequent visits to an oral health care provider $(12,13)$. According to the Centers for Medicare and Medicaid Services and the Bureau of Economic Analysis, as of 2014, the U.S. has spent $\sim 113$ million dollars on dental care expenditures. From an ethnic standpoint, it was determined that the disease has the highest prevalence in Hispanics, due to the association with the lowest levels of education and a higher population of smokers (12). The oral cavity represents a unique environment, as it is a major gateway to the rest of the human body (14). It is constantly utilized for passage of air and food, which will travel through 
the body systemically to its target site, however, microorganisms present will also use this transport to spread along the contiguous epithelial surfaces (14). Therefore, clear evidence is now available linking periodontitis to a number of systemic infections and diseases, such as cardiovascular disease, stroke, preterm birth, diabetes and pneumonia $(2,14)$.

The prevention and reduction of periodontal diseases in the U.S. population is part of a national health initiative, Healthy People 2020, and is considered a strategic objective for the CDC (12, 15, 16); U.S. Department of Health and Human Services. Healthy People 2020: Oral Health Available at: http://www.healthypeople.gov/2020/topicsobjectives2020/overview.aspx?topicid=32). The oral cavity is accessible for study, as the dental plaque containing the bacterial species and the gingival crevicular fluid containing the host-derived cytokines and chemokines can be sampled non-invasively $(1,17-23)$. Sampling of the gingival crevicular fluid can be accomplished by inserting a piece of filer paper, a paper point into the crevice and dental plaque is obtained through scraping of the tooth surface with a hand-held dental tool, a curette $(1,6,24-29)$.

The biofilm structure formed by the community of bacteria in the oral cavity provide a safe haven for residing in this environment, where there is resistance to antibiotics as well as host evasion of important immune cell responders like neutrophils and macrophages, due to the sticky and impenetrable nature of the biofilm $(5,30-32)$. While most human microbiome systems are in a homeostatic state, diseases are caused when there is a shift in the microbial population leading to dysbiosis. The oral microbiome is more often found in a state of dysbiosis, as is evident by the disease it causes in a majority of people in their lifetime, as evidence supports half the U.S. population age 30 or older suffers from some form of periodontal disease $(12,33-35)$. Culture-dependent studies performed to characterize the oral microbiome implicated three bacterial species, Porphyromonas gingivalis, Tannerella forsythia, and Treponema denticola, were associated with disease development $(1,36,37)$ However with 
culture-independent sequencing studies, it has been determined there are many more potential pathogens associated with disease. To maintain homeostasis and a healthy gingival tissue, a delicate balance between the host and the indigenous oral microbial community is required (5, 38). Certain diseases caused by the shift in members comprising the oral microbiota, where there is an increase in the pathogenic bacteria and a decrease in the beneficial commensals, lead to the current 'microbial shift' hypothesis, which explains the concept of a dysbiotic, diseasefavoring state (5). These states of dysbiosis are found to be associated with diseases other than periodontal disease, including: inflammatory bowel disease, bacterial vaginosis, gastroesophageal reflux disease, and otitis media, the common underlying mechanism being inflammation $(5,39-41)$. Furthermore, this shift also influences the host's response, as now a potent and unresolving inflammatory environment is created, which leads to tissue destruction and eventually alveolar bone loss $(5,42)$. The key to the maintenance of a healthy periodontium is the expression of vital host defense mediators (1).

It is estimated that there are over 700 bacterial species present in the oral cavity, with more than half of them remaining uncultivated $(5,43)$. It is a common misconception that those bacterial species that are easily cultivated and often present in high quantities in the oral cavity are solely responsible for the development of periodontal disease and those species are often the focus of further studies $(1,18,44-49)$. However, it is now becoming apparent that pathogenicity and virulence properties are most important when determining which bacterial species are relevant in disease development, as those difficult to culture or in low quantities may represent an important species in disease progression that was previously excluded from studies. It was previously thought that gram positive bacteria were associated with health, while gram negative bacteria were associated with disease, however that concept is being reevaluated with the assessment of newly identified organisms, like Filifactor alocis $(F$. alocis) $(5,50)$. 


\section{Filifactor alocis.}

Species like $F$. alocis are recently identified due to the large expansion in knowledge of culture-independent approaches, like 16S ribosomal RNA (rRNA) gene-based molecular cloning techniques, which allows for the identification of species from their DNA directly (14, 44). Due to these new identification methods being developed, previously overlooked bacterial species are coming to the forefront in study and are observed to be an important player in disease development $(14,44,51,52)$. Utilizing these culture-independent techniques promises further expansion of the diversity of the oral cavity and a deeper understanding of the nature of disease development by biofilm communities $(14,53)$. F. alocis is one of those overlooked and underappreciated species, as it was previously excluded from studies due to its slow growth and difficulty in detection by conventional culture-based methodologies (44).

F. alocis is a gram-positive, asaccharolytic, fastidious, rod that grows under obligate anaerobic conditions $(2,54)$. This bacterium was first isolated from clinical patients afflicted with gingivitis and periodontitis in 1985 and given the name Fusobacterium alocis, before being reclassified in 1999 to Filifactor $(2,55)$. This bacterium is now determined to be related to Eubacterium, as opposed to a fusiform-like species $(54,56)$. F. alocis has been associated with several oral conditions like peri-implantitis, and endodontic infection, however, it is now known to be linked to the development of periodontal disease. It is found to be significant to the pathogenic biofilm produced in a disease setting, can establish synergistic relationships with other oral pathogens, and possesses potential virulence properties like oxidative stress resistance, that allow it to effectively colonize, survive and out-compete other periodontal pathogens $(2,57)$. Studies performed by Dahlen et al have determined F. alocis a species relevant in routine diagnostics for periodontal disease and this bacterium is also found to be associated with other oral pathogens such as P. gingivalis, Streptococcus gordonii (S. gordonii), Fusobacterium nucleatum (F. nucleatum), and Aggregatibacter actinomycetemcomitans (A. 
actinomycetemcomitans) $(54,58)$. In patients with periodontal disease, $F$. alocis is often present and it has been proposed that this bacterium should serve as a diagnostic indicator of the disease $(2,50,52,54,59)$. Moreover, $F$. alocis is absent in healthy patients (2).

F. alocis is known to survive and persist in the periodontal pocket through the use of its virulent properties: resistance to oxidase stress, stimulated growth in conditions of oxidative stress, induction of proinflammatory cytokines, and pro-apoptotic local infection $(2,44,60)$. This bacterium has been studied in gingival epithelial cells, where it triggers apoptosis in a caspase-3-dependent manner, through suppression of MAPK/ERK kinases 1 and 2 (MEK1/2) activation $(2,44)$. Additionally, in mouse model studies by Wang et al F. alocis produces a local pro-apoptotic infection affecting the gingival epithelial cells, that can be cleared rapidly due to the massive influx of neutrophils $(2,61)$. As an asaccharolytic organism, F. alocis thrives on the amino acids for nutrition, survival and virulence, which it obtains through degradation of proteins of other bacteria and host tissues, mainly relying on arginine $(2,62)$. This bacterium produces proteases and neutrophil-activating protein A which are found to be upregulated when internalized, as observed in studies with gingival epithelial cells $(63,64)$. The $F$. alocis genome also includes another important mechanism that uses arginine, citrulline synthesis $(2,62)$. The process of protein citrullination is known to be an important post-translational modification linked to systemic complications, like the development of rheumatoid arthritis, as this process is a crucial epigenetic regulatory mechanism that can cause an ongoing state of inflammation $(2$, 65 ). It has been reported that $F$. alocis has a highly efficient mechanism of protein-sorting/transport system, which is due to its large number of membrane proteins $(2,64)$. This system could be what is responsible for facilitating the efflux of reactive oxygen species (2). This bacterium is able to provide for its own nutritional needs, as the genome contains many important proteases $(2,64)$. Arginine is an essential amino acid for the growth and survival of F. alocis and to its benefit, there are large amounts of arginine present in the periodontal pocket 
$(2,62)$. Additionally, F. alocis is abundant in arginine metabolism proteins which allow it to optimally utilize that arginine (2).

As cytokine homeostasis is necessary for keeping a healthy periodontium, the disruption of this homeostasis is implicated in tissue destruction and further, periodontal disease $(44,66)$. F. alocis induces the release of pro-inflammatory cytokines like interleukin 1 beta (IL-1 $\beta$ ), interleukin 6 (IL-6) and tumor necrosis factor alpha (TNF- $\alpha$ ) in studies performed on gingival epithelial cells (44). These identified cytokines are important as they are known to stimulate osteoclasts, increase the resorption of alveolar bone and contribute to tissue degradation through inflammatory mediators like matrix metalloproteinases $(44,66-69)$.

Additionally, it has been observed that $F$. alocis can partake in the formation of a biofilm community in both endodontic and periodontic infections $(2,70)$. It was determined through in vitro studies by Wang et al that $F$. alocis can participate in the development of a biofilm community with a variety of bacteria that have varying degrees of pathogenic potential including: Streptococcus gordonii, Fusobacterium nucleatum, P. gingivalis and Aggregatibacter actinomycetemcomitans $(2,61)$. Additionally, in studies performed on gingival epithelial cells by Moffatt et al, it was determined that $F$. alocis can form biofilms in vivo (44, 70). The interaction of F. alocis with other oral pathogens indicates there could be a mutually beneficial relationship between the bacteria, allowing each to survive and evade host detection mechanisms (2). For instance, the interactions between $F$. alocis and $P$. gingivalis results in the upregulation of factors responsible for making both bacteria more virulent $(2,60,71)$. In biofilm studies, it was determined that $F$. alocis forms a mutually beneficial relationship when in a two-species community with $F$. nucleatum, which is not observed for other oral pathogens S. gordonii, A. actinomycetemcomitans and $P$. gingivalis (63). Interactions between $F$. alocis and $S$. gordonii revealed that $S$. gordonii strongly inhibits colonization of $F$. alocis (63). This demonstrates that $F$. alocis may not be effective in colonizing regions of dental plaque that are 
streptococcal rich (63). F. alocis and A. actinomycetemcomitans showed that their interactions are strain-dependent, as the strain ATCC 35896 showed mutual accumulation of both species while strain D-62D did not stimulate accumulation of either bacterium (63). F. alocis and $P$. gingivalis will physically interact initially and then $P$. gingivalis demonstrates a highly inhibitory phenotype towards F. alocis (63).

Neutrophils.

Neutrophils were first described by Paul Ehrlich in the late $19^{\text {th }}$ century as a subpopulation of leukocytes characterized by their "polymorphous nucleus", which generally has 3-4 lobes (72). Their tendency to retain neutral dyes, lead to the name neutrophil or polymorphonuclear cell (PMN) (72). Due to their uniquely lobulated nucleus, they are easily identifiable by Wright staining (73). Initially, it was believed that these cells were present in large numbers at sites of infection due to their ability to serve as cellular shuttles in delivering bacteria to tissue sites (72).

Neutrophils are in the granulocyte family and produced in the bone marrow where they first represent a population of pluripotent hematopoietic cells, which differentiate into myeloblasts, cells that are committed to future development into a granulocyte $(72,74)$. Their production is extensive as $\sim 1-2 \times 10^{11}$ cells per day in a normal adult can be generated in the steady state $(73,74)$. Granulocyte colony stimulation factor (G-CSF) is an essential component in regulating the production of neutrophils in order to meet their demands in the context of an infection, however when the neutrophils have reached their site of interest, the production of this factor is reduced (74).

Neutrophils are among the shortest-lived cells in circulation in the human body, which is beneficial to the host for mounting an initial potent antimicrobial response, however, due to the toxic environment they create in response to their target, they should be effectively undergo 
apoptosis and clearance by macrophages. In vitro, neutrophils do not undergo proliferation and are estimated to have a half-life of 10-12 hrs (75). However, in vivo their life span is extended due to signals that delay their programmed cell death such as adhesion, transmigration, hypoxia, microbial components and cytokines $(75,76)$. Additionally, neutrophils will survive up to 1-5 days at the tissue site of infection, where they can persist due to presence of immunoregulatory cytokines and bacterial-produced factors. This prolonged lifespan allows for neutrophils to more effectively respond to injury or inflammation, and further promotes interaction with other cell types such as macrophages, mesenchymal stem cells, dendritic cells, natural killer cells, B and T lymphocytes (75).

In humans, neutrophils are the most abundant of the white blood cells in circulation (72). They are first responder cells in the innate immune response and are critical for host defense, as demonstrated in patient studies where life-threatening conditions develop when neutrophils are impaired either in number or function $(74,75,77,78)$. Once neutrophils are depleted at the site of injury or inflammation and neutropenia is sensed, granulopoiesis is signaled in order to replenish the neutrophil population and ensure the host will further function in combating microbial barrage (73). Interestingly, it has been shown that microbiota can help to regulate initiation of granulopoiesis, as studies found that germ-free mice are severely neutropenic $(73,79)$.

Human neutrophils constitutively express all Toll-like receptors (TLRs), except TLR3 $(72,73)$. TLRs are among the most recognized family of molecules known for their ability to be presented on a variety of immune cell types and recognize numerous stimuli (72). Upon encountering pro-inflammatory cytokines or bacterial stimuli, there is an enhanced expression of TLR4 or TLR2, respectively, on the surface of human neutrophils (80-83).

\section{Benefits to using human neutrophils for our studies.}

Neutrophils are terminally differentiated, they cannot undergo further growth in tissue culture conditions or be genetically modified $(72,73)$. Additionally, immortalized neutrophil-like 
cell lines, such as HL-60s, are rarely capable of encompassing the vast functions of neutrophils that are found in primary cells (72). Although most often used for in vivo neutrophil studies, mouse neutrophils also possess limited capabilities in function and also only about $30 \%$ are found in circulation compared to about $70 \%$ in humans (72). Mouse neutrophils do not fully mimic human neutrophils and are different in many capacities: function, morphology, and physiology (73). Additionally, one of the major issues impeding neutrophil research is the lack of genetic tools (84-90). It has been demonstrated that in vivo mouse neutrophils can be depleted via antibodies, however this depletion is only transient, as low neutrophil counts will initiate the production of new neutrophils to be developed (73). Neutrophils can be specifically depleted from mice using Ly6G monoclonal antibody (91). Additionally, using a S100a8(MRP8)cre:ROSA-YFP knock-out mouse results in a neutrophil specific depletion (92). However, it is necessary to interpret cell line and mouse neutrophil studies carefully, as the extrapolation to human physiology and pathophysiology may not be directly translational $(84,93-96)$.

Neutrophil migration- from the blood stream to the site of injury, infection, or inflammation.

Neutrophils remain in circulation until different signals from cytokines, chemokines, and bacteria stimulate them to initiate their migration towards the site of inflammation. An extensive process referred to as 'Rolling- Adhesion - Transmigration' is responsible for neutrophil migration out of circulation to sites of infection or inflammation. The 'Rolling' step refers to the initial attachment of neutrophils to the endothelial cells and occurs when cells are stimulated with cytokines produced during an infection such as TNF- $\alpha$, IL-1 $\beta$, and interleukin 7 (IL-7) (74, $97-$ 99). This chemokine production stimulates expression of selectins (P- and E- selectin), integrins such as intracellular adhesion molecules (ICAMs) and vascular cell adhesion molecules (VCAMs) on the luminal surface of the endothelial cells which can bind to the selectin and integrin ligands located on the neutrophils $(74,98,100)$. Firm adhesion is the second step in the process and ensures the cessation of rolling. This requires an activated endothelium to bind to 
neutrophils through adhesion molecules (LFA-1). Once the endothelium is activated, it in turn secretes cytokines such as interleukin 8 (IL-8) and macrophage-inflammatory protein 2 (MIP-2), which are known to activate neutrophils (74). With the induction of firm adhesion, neutrophils will next undergo polarization upon the leading edge lamellipodium, allowing for receptors for phagocytosis and chemokine signaling to be expressed (74). The final step is transendothelial migration whereby neutrophils can penetrate the endothelium by either transcellular (penetration of a single endothelial cell) or paracellular migration (penetration between endothelial cells) (74). The transcellular route is mediated by Mac- 1 and is only responsible for the migration of $\sim 20 \%$ of neutrophils $(74,101)$. Once neutrophils have successfully migrated through the endothelium, they utilize proteases, collagens, laminins (elastase), matrix metalloproteases (MMP8, MMP9) in order to penetrate the basal membrane $(74,102)$.

Neutrophils operating in the tissues are now more activated than when their journey began in the blood, making the transition from circulating cells to tissue phagocytes (103). Now active, neutrophils induce their transcriptional program, which results in production of chemokines like IL-8 and growth-regulated oncogene alpha (Gro- $\alpha$ ), which are known to signal recruitment of other inflammatory cells, like macrophages and $\mathrm{T}$ cells, to the site of injury or inflammation $(74,104-106)$. While neutrophil activation is essential for a defensive response to a variety of microorganisms, it is also essential that their activation be tightly regulated to prevent host tissue damage. Neutrophils employ a very efficient intracellular negative feedback loop to help in regulation of their activity, mostly through factors like immunoreceptor tyrosine-based inhibitory motifs (ITIMs), signal inhibitory receptor on leukocytes-1 (SIRL-1), carcinoembryonic antigen related cell adhesion molecule 1 (CEACAM1), cluster of differentiation 300a (CD300a) and suppressor of cytokine signaling 3 (SOCS-3) (74, 107-111). Neutrophils operate in two waves of activation and recruitment following transendothelial migration. First, neutrophils found at the tissue site of injury or inflammation begin to respond and this is followed by a second wave of neutrophils that can be recruited from a distance as far as $200 \mu \mathrm{m}$ from the site (103). 
Neutrophil-neutrophil signaling makes it possible for distant cells to be recruited through chemoattractant signaling cues, further strengthening the capacity of the neutrophils to effectively respond at the tissue site (103).

When presented with a chemotactic stimulus, neutrophils migrate very quickly (up to 12 $\mu \mathrm{m} / \mathrm{min}$ ), which allows them to reach their target site within $3 \mathrm{~h}$ of the initial onset of injury or inflammation $(112,113)$. When neutrophils encounter a chemoattractant, they polarize and polymerized actin accumulates at the leading edge (114). Neutrophils have the ability to rapidly orient themselves and move through anterior extension and posterior contraction and retraction, upon exposure to a chemoattractant (115).

In comparison, other white blood cells like monocytes take at least $12 \mathrm{~h}$ to be fully recruited $(112,116)$. Directional movement of leukocytes is necessary for a functional response to a variety of inflammatory signals (117). Leukocytes participate in directional movement toward sites of injury and inflammation by deciphering a chemoattractant gradient, a process referred to as chemotaxis $(114,117-120)$.

As neutrophils express receptors for chemotactic factors, they are able to effectively detect gradient intensity and move toward the source with directionality $(114,117,121)$. Cells with chemotactic functions are able to sense slight changes in the concentration of a chemoattractant, sensing changes $1 \%$ over the length of a single cell $(8-12 \mu \mathrm{m})(119,122)$. Once the neutrophils encounter a high concentration of chemoattractants, they further initiate the process of phagocytosis and killing (118).

Since neutrophils are exposed to many chemoattractants that are released at various locations including the vascular endothelium, interstitial cells and the site of infection, it is necessary for them to assimilate and prioritize their response $(120,123,124)$. They can respond to signals from both intermediary chemoattractants (IL-8, platelet activated factor, chemotactic 
cytokines, leukotriene B $\left.4\left(\mathrm{LTB}_{4}\right)\right)$ and end target cellular chemoattractants (formyl-methionylleucyl phenylalanine (fMLF), complement fragment C5a, C3a, plasminogen activator), which guide them to a generalized region and then further to a more specific site, where they will encounter their target $(118-120,123)$. Ultimately, neutrophils will dissociate from the intermediary chemoattractants and migrate toward the end-target chemoattractants of bacterial origin $(119,125)$. Although the chemoattractants vary significantly in their structure, the receptors for these molecules are all members of the seven-transmembrane helix receptor family which operate through to heterotrimeric G proteins, which further activate downstream pathways responsible for cytoskeletal arrangements and chemotactic functions $(119,120,123)$.

Phagocytosis and killing mechanisms employed by neutrophils.

Neutrophils serve as highly efficient phagocytes through the receptor-mediated process of phagocytosis, where a particulate is internalized into a phagosomal compartment (72). Phagocytosis can occur through the recognition of pattern-recognition receptors (PAMPs) or mediated through opsonins (72). Initially, upon internalization of the particle, the phagosomal compartment is benign; it is not until phagosome maturation that the phagosome exhibits lethality (72). This maturation process is initiated when neutrophil granules fuse with the phagosome and release their antimicrobial contents (72). Simultaneously, the nicotinamide adenine dinucleotide phosphate (NADPH) oxidase complex assembles at the phagosomal membrane, leading to the production of reactive oxygen species (ROS) (72). Using these two antimicrobial mechanisms, neutrophils create an inhospitable environment for most pathogens, which results in bacterial cell death (72). However, some pathogens have evolved mechanisms that allow for interference with the engulfment process or ensure their survival inside neutrophils through modulation of phagosomal maturation, and creation of a more hospitable phagosomal environment $(72,126-130)$. 
Neutrophils are efficient and effective responders to sites of injury or inflammation, where they target microbes for elimination (72). In the most basic sense, they are in charge of killing the bad without causing too much damage to the host (72). It is believed that the degranulation and ROS production may function in concert and potentiate one another, leading to a highly efficient killing process employed by neutrophils (73). Currently, it is still unclear whether neutrophils are triggered differently by various stimuli to employ a certain antimicrobial killing mechanism (73).

\section{a) Neutrophil granules.}

One of the most important features of neutrophils is their granules, a hallmark of granulocytes (neutrophils, eosinophils, basophils) (74). Granules are membrane vesicles that are composed of a wide variety of proteins, peptides and antimicrobial components and function either in vesicle delivery to the cell membrane for exocytosis or fusion with the bacterialcontaining phagosome, along with the assembly of the NADPH oxidase complex, for killing internalized microbes. Neutrophils possess three main types of antimicrobials: 1) cationic peptides and proteins which can bind to microbial membranes, 2) enzymes, and 3) proteins involved in deprivation of essential nutrients for microorganisms $(72,131)$. They are stored in a specialized organelles that allows for the neutrophil to safely transport potent antimicrobial components through the blood stream to a site of infection or injury (72).

In humans, there are four granule subtypes that are released in a hierarchal fashion, based on their density and content (Fig 1-1) $(72,74)$. The primary or azurophilic granules and the secondary or specific granules are crucial for their involvement in providing the neutrophil with a potent antimicrobial arsenal. The azurophilic granules, the largest of the granules and formed first during neutrophil maturation are designated the most potent based on their abundance of molecular weaponry, most notably proteins such as myeloperoxidase (MPO), and neutrophil elastase (NE) (72). The next to be formed during the maturation process are the secondary or 
specific granules, which contain a variety of antimicrobial compounds, the most important being lactoferrin and lysozyme (72). Additionally, the specific granules contain cytochrome b558, which is a heterodimer composed of the cytosolic NADPH oxidase components gp $91^{\text {phox }}$ and p22 $2^{\text {phox }}$ Following specific granules are the tertiary or gelatinase granules, which function mainly for storage of metalloproteases, such as gelatinase and leukolysin (72). Lastly, the secretory granules are formed through endocytic processes in the late states of neutrophil maturation and their contents consist of plasma-derived proteins like albumin and membrane-bound molecules (72).

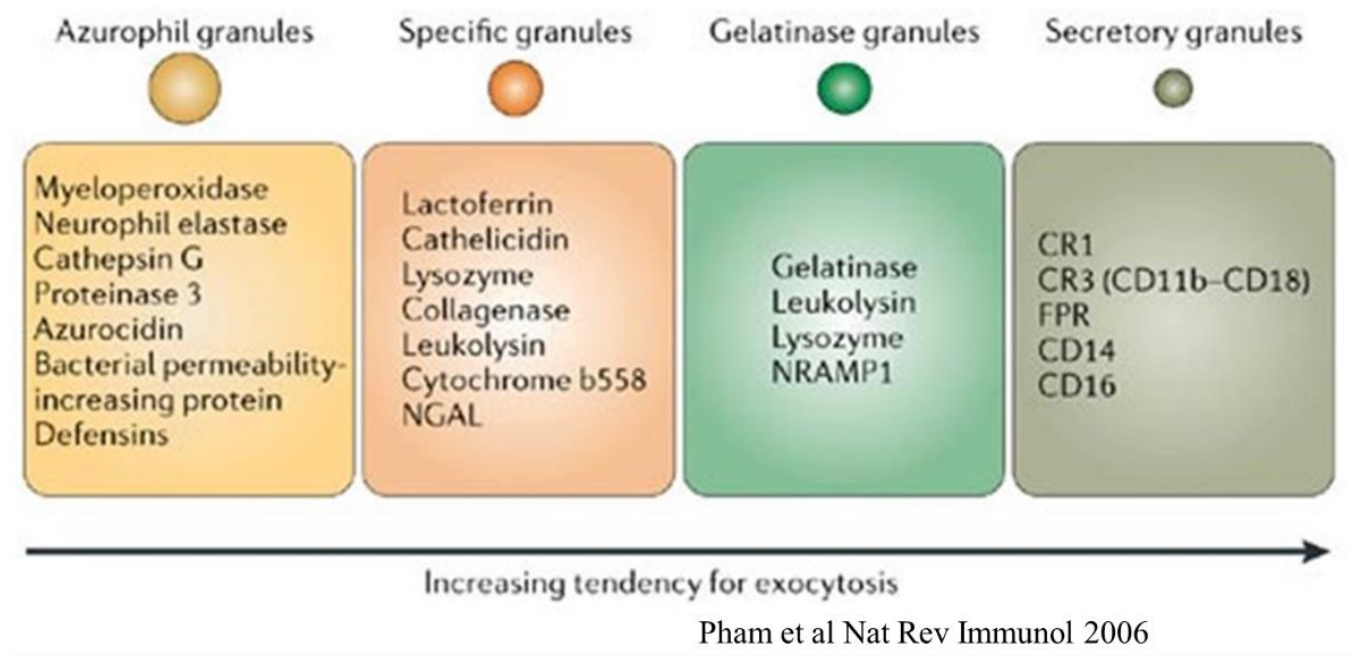

\section{Figure 1-1. Neutrophil granules and their hierarchal exocytosis.}

As neutrophils undergo activation, the granules are mobilized to either fuse with the plasma membrane or the bacteria-containing phagosome, which allows for efficient elimination of both intracellular and extracellular pathogens $(72,112)$, as granules can be released internally in the phagosome, or externally at the surface of the plasma membrane (75).

\section{b) Oxidative burst response.}

Once neutrophils are activated through recognition and binding of bacteria or bacterialderived factors to their surface, they undergo a process called respiratory burst, which is 
characterized by the production of ROS (72). If the stimulus is particulate, it will be internalized to the phagosome and the intracellular respiratory burst response will occur inside the phagosomal compartment. However, if there is soluble stimuli present or the neutrophil cannot effectively internalize the stimuli, an extracellular respiratory burst response will be initiated on the cell membrane. Respiratory burst is initiated following the assembly of the NADPH oxidase complex, which consists of two membrane components for specific granules, gp9 $91^{\text {phox }}$ and $\mathrm{p} 22^{\text {phox }}$, and three cytosolic components $\mathrm{p} 47^{\text {phox }}$ and $\mathrm{p} 67^{\text {phox }}$ and $\mathrm{p} 40^{\text {phox }}$ along with GTPase Rac2 (132). In resting neutrophils, the components will be found either in the cytoplasm or the membrane, however upon stimulation or activation, $\mathrm{p} 47^{\text {phox }}$ is phosphorylated and moves to the phagosome or plasma membrane $(132,133)$. Following the translocation of $\mathrm{p} 47^{\text {phox }}$, translocation of both $\mathrm{p} 67^{\text {phox }}$ and $\mathrm{p} 40^{\text {phox }}$ is now facilitated and associates with membrane bound $\mathrm{p} 22^{\text {phox }}$ which interacts with GTP-bound Rac2 (Fig. 1-2)(132-137).

RESTING

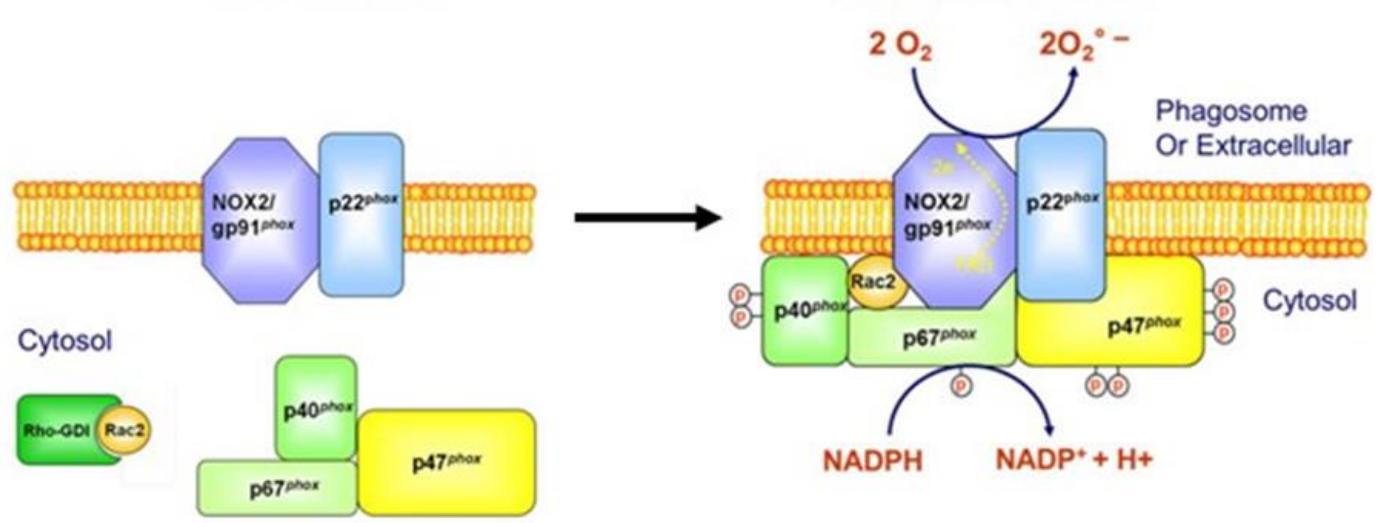

Adapted from El-Benna et al Immunol Rev 2016

Figure 1-2. NADPH oxidase activation and assembly leading to the respiratory burst response.

Upon assembly of the NADPH complex at either the plasma membrane or the phagosomal compartment, the reactive oxygen cascade begins with the reduction of molecular oxygen to superoxide (72). Next, superoxide will rapidly dismutate, forming hydrogen peroxide 
(72). In the phagosomal compartment, MPO is known to react with hydrogen peroxide to form a variety of reactive species, most notably hypochlorous acid, which is highly reactive and potent leading to a strong antimicrobial product (72).

Without the assembly of the NADPH oxidase complex, no detectable ROS is able to be produced (132). Therefore, it has been reported that intracellular pathogens have evolved to incorporate strategies allowing them to inhibit assembly and/or activation of the NADPH oxidase complex, further allowing them to escape killing by ROS production of neutrophils $(132,138$ 140). It has been determined that ROS is important for antimicrobial activity in neutrophils, through the studies of patients with chronic granulomatous disease (CGD) (72). Neutrophils from CGD patients are fully functional when provided exogenous hydrogen peroxide, as normal antimicrobial activity is observed in this context, indicating the specific defect in ROS production (73). Patients suffering from CGD are unable to mount an efficient respiratory burst response by neutrophils, as they have a defect in one of the NADPH oxidase components, leading to inefficient assembly and activation, critical per-cursor steps to reactive oxygen species (ROS) production $(73,141)$. The severity of CGD depends on the genetic mutation present in the patient, but in worse cases, the activity of the NADPH oxidase complex can be as low as $0.1 \%$ of a healthy individual $(73,141)$. About two-thirds of CGD patients are effected by recessive mutations in the X-linked CYBB gene which encodes for gp9 ${ }^{\text {phox }}(142)$. The remaining patients have autosomal defects in genes for $\mathrm{p} 22^{\text {phox }}(\mathrm{CYBA}), \mathrm{p} 47^{\text {phox }}(\mathrm{NCF} 1)$ or p67 $7^{\text {phox }}(\mathrm{NCF} 2)(142)$. These gene defects result in the absence of the encoded protein responsible for superoxide production (142).

The NADPH oxidase complex can assemble at the cell membrane if the neutrophil encounters a soluble (ex: fMLF) stimulus or at the phagosomal membrane when encountering a particulate stimulus (ex: bacteria or yeast) and further will generate superoxide release into the extracellular environment or phagosomal compartment, respectively $(143,144)$. NADPH oxidase 
activity takes place in three stages: 1) initiation by assembly and activation, 2) maintenance of activation, 3) deactivation of the response through component disassembly (145). NADPH oxidase activity and the induction ROS response depends on the assembly and activation of the complex, however it is also important, most importantly to the host, to deactivate and disassemble $(135,145)$. Neutrophils that cannot effectively perform phagocytosis will undergo extracellular ROS production, releasing their antimicrobial reactive oxygen species in the extracellular space instead of the phagosome $(146,147)$.

\section{c) Neutrophil extracellular traps.}

Neutrophil extracellular traps (NETs), first described by Brinkmann and Zychlinsky in 2004, are a newly appreciated mechanism of killing, which occurs when PMNs release their decondensed chromatin extracellularly $(72,148)$. NETs operate through two major mechanisms to perform their antimicrobial functions, trapping bacteria and preventing their spread both locally and systemically and killing through the use of embedded antimicrobial peptides (149, 150). Their fibrous structure is known to contain histones, antimicrobial granular and cytoplasmic proteins, which can trap and kill microbes (72). NETs have been proposed to function in both trapping and killing various microbes as well as activating and recruiting other immune cells, via exposure to a concentrated form of antimicrobials that decorate their structures (73). Although trapping and killing would be the most effective strategy, merely trapping bacteria could serve beneficial in reducing spread of infection (103). Previous studies have shown that NETs are significant in limiting bacterial dissemination $(149,151)$.

NETosis, the name designated for this process, is thought to be an alternative mechanism to death by apoptosis, which ensures resolution of inflammation, or by pyroptosis, secondary necrosis or necroptosis, which promote proinflammatory cytokine release and activation of macrophages $(103,152,153)$. More recently, studies have shown that neutrophils can undergo 'vital NETosis', whereby NETs are produced while neutrophils remain intact, alive, can still 
undergo active phagocytosis and can migrate $(103,154,155)$. While it was originally believed that NET formation is dependent on the NADPH oxidase activity, an ROS-dependent process, new research has led to the idea that NET formation can also occur via an ROS-independent mechanism $(84,148,156,157)$.

A variety of stimuli have been associated with NET release: nitric oxide, cytokines, microbes and microbial products, antibodies, statins, platelets and antimicrobial peptides (148, 149, 158-164). It has been determined by many studies that NETs can be induced and/or kill a large range of pathogens including bacteria (both Gram-positive and Gram-negative), fungi, protozoan parasites and viruses (165-167). Also, NETs can be formed in the presence of physiological inducers, like lipopolysaccharide (LPS) non-physiological agents like phorbol 12myristate 13-acetate (PMA) (168). The composition of NETs includes nuclear chromatin, nuclear citrullinated histones and also granular antimicrobial proteins, such as NE and MPO, which provide an extracellular matrix for trapping and killing pathogens (169-171).

Excessive NET formation is associated with disease development $(149,172)$. Their release must be highly regulated, as this process has been linked to harmful effects on the host, including tissue damage and even the development of autoimmunity $(72,159,161,173-175)$. Although the antimicrobial granule proteins play a vital role in bacterial killing, these proteins may also cause unnecessary damage to host tissue and potentially chronic inflammation, if not cleared from the infection site $(149,176)$. A link has been established between NET formation and autoimmune disease, like with systemic lupus erythematosus (SLE), which is caused by the formation of autoantibodies targeting chromatin and neutrophil components, and results in symptoms of fatigue, joint pain, rash and fever (72). The high levels of proinflammatory cytokines found in patients with autoimmune disease are proposed to sensitize neutrophils to form NETs (72). Additionally, the presence of circulating autoantibodies may induce a switch from PMN apoptosis to NETosis (72). The contents released during NET formation may serve as 
a source of autoantigens, which has been implicated to lead to complications in the development of autoimmune diseases like rheumatoid arthritis, SLE and vasculitis $(73,103)$.

The role of neutrophils in periodontal disease.

The innate immune response, driven by neutrophils, is of critical importance to the maintenance of periodontal health in the host (1). It has been estimated that $\sim 30,000$ neutrophils circulate through the periodontal tissue per minute $(1,177,178)$. Neutrophils are found in large numbers in the gingival crevice as well as in the epithelial tissue, designating them as the major effector cell of the periodontium (179-183). It has been proposed that neutrophils form a 'wall' of protection for their host, which is formed along the gingival epithelium and the pathogen-rich biofilm community $(179,181,182)$.

Neutrophils will be present in health, producing a low-level inflammatory state, stimulated through the presence of resident oral microbiota, where they serve as patrolling agents ready to respond if needed, until they will undergo eventual cell apoptosis and clearance by macrophages (184-186). In the inflamed periodontal tissue, chemotactic factors such as IL8 , as well as bacterial derived products, fMLF, will be abundant and guide neutrophils from the blood vessels through the gingival tissue towards the periodontal pocket $(179,187,188)$.

In the context of periodontal disease, neutrophils are impaired in their ability to perform chemotaxis, which impacts their migration to the site of bacterial presence in the oral cavity, allowing for bacterial persistence and survival in the host $(184,189)$. Additionally, these neutrophils are maintained in a 'primed' activation state both in the oral cavity and in the blood stream, which could have further implications in the association of oral disease with the development of other chronic inflammatory diseases like atherosclerosis, diabetes, and rheumatoid arthritis (184). Priming agents (e.g. fMLF, TNF- $\alpha$ ) of bacterial and host origin serve to pre-activate or 'prime' neutrophils to form a more robust response upon encounter with a 
secondary stimulus (190). In the context of disease, primed neutrophils can be critical to ensuring a robust effective killing response, however an excessive priming can lead to unnecessary collateral host tissue damage.

There is a dynamic cross-talk between periodontal pathogens and neutrophils in the development and progression of periodontal disease $(179,182,191-193)$. Neutrophils that are impaired in function, show increased susceptibility to bacterial infection and development of disease (179). However, if on the other hand, neutrophils are in a hyperactivated state, this could lead to chronic and unresolved inflammation and bacterial persistence and survival in the oral cavity, which would greatly damage host tissues while providing a constant nutrient-rich environment for the bacteria $(179,182)$.

Due to the importance of neutrophils in the innate immune response to a variety of stimuli, it is anticipated that in response to these cells, pathogens have developed means to interfere with and prevent chemotaxis. In studies performed by Darveau, it was observed that in gingival epithelial cells, $P$. gingivalis does not induce IL-8 production and it is known to antagonize IL-8 production which leads to suppression of neutrophil migration and further allows for bacterial overgrowth to occur $(44,194)$. Additionally, once homeostasis is disrupted, there is a reduction in the secretion of IL-8, which further impairs neutrophil recruitment to the site of infection and allows for growth and persistence of bacterial species (1). P. gingivalis also contains serine phosphatase, SerB, which operates to prevent granulocyte recruitment to periodontal tissues $(179,195)$.

It has been suggested that NETs play an extensive role in both periodontal health and disease (149). In the context of periodontal disease, exacerbation of disease may occur if there is an excessive NET production $(149,196)$. On the other hand, ineffective NET production could be a contributing factor in periodontal disease development (149). NETs have previously been 
observed to be present in both purulent exudates from the gingiva as well as in gingival crevicular fluid from patients suffering from chronic periodontitis $(149,197)$. Using immunofluorescent imaging, NETs have been visualized in gingival connective tissues at inflammatory sites of infection, however, in healthy patients, less NET formation is detected (149).

Neutrophil specific disorders play a role in periodontal disease development.

The presence of fully functional neutrophils is crucial for ensuring periodontal health, as patients suffering from the neutrophil disorders listed below are plagued by the development of periodontitis (179). Overall, genetic diseases responsible for impaired neutrophil functions are found in less than 1 in 200,000 individuals, which can underscore the importance and relevance of these cells function in antimicrobial defense (73). However, it is important to point out that impairment of neutrophil functions leads to impairment of other immune cell types and greatly enhances the chance for the patient to succumb to a variety of bacterial and fungal infections (73). Additionally, numerous syndromes causing defects of neutrophil number or function show patients invariably develop periodontal disease $(1,198)$.

Chronic neutropenia, meaning abnormally low levels of circulating neutrophils, is linked with the development of persistent and chronic bacterial infections, and most importantly for our studies, the development of aggressive periodontitis $(198,199)$. In neutropenic patients, the most common pathogen found is $S$. aureus, which is easily eliminated from healthy individuals (73, 200, 201). Additionally, fungi like Candida and Aspergillus species are known to be problematic when one or all of the neutrophil's defense mechanism are deficient $(73,202)$.

Patients that suffer from leukocyte adhesion deficiency type 1 and type 2 (LAD1, LAD2) have congenital deficiencies in neutrophil number or transit and will consistently develop periodontal disease $(1,203-206)$. LAD1 is fatal without bone marrow transplant (198).

Chediak-Higashi syndrome effects the lysosomal regulator gene (LYST) and results in increased susceptibility to infection as well as large inclusion bodies seen in the bone marrow 
$(198,207)$. Additionally, in patients with Chediak-Higashi syndrome, an aggressive and unresponsive form of periodontitis develops $(198,208,209)$. Mutations in the cathepsin $\mathrm{C}$ gene (CTSC) cause Papillon-Lefèvre syndrome, which impairs activation and recruitment of leukocytes, as well as their degradation of microbes inside the phagosome (198). PapillonLefèvre syndrome can cause pre-pubertal periodontitis and if enzymatic defects associated with this condition are left untreated, severe periodontitis affecting both dentitions will develop (198).

\section{Treatment of periodontal disease.}

There are many challenges associated with treating periodontal disease: salivary flow as well as large differences among individuals $(5,210)$. The oral microbiota shifts with changes in diet, hygiene and age of individuals and therefore, it is difficult to identify a 'typical' healthy vs. diseased individual, as there are many flavors for either context $(5,43,211-213)$.

The overall goal of periodontal therapy is to reduce and eliminate pathogenic bacteria while maintaining the community of commensal bacteria $(57,214,215)$. The current standard treatment for periodontitis includes a combination of scaling and root planning with an antibiotic regimen (5). Although this method may prove effective against known pathogenic bacteria, it may not be effective against unusual and yet-to-be-cultivated species, leading to their persistence and growth in the oral cavity and a state of disease $(57,216-219)$.

However, antibiotic therapy is usually unsuccessful due to the fact that biofilms are resistant to them $(5,30)$. Another important point of consideration is that a large number of oral bacteria have not yet been identified and antibiotic treatment may only be successful in treating some of the bacteria residing in the oral cavity $(5,43)$. Further, using antibiotic treatment not only impacts the bacteria, but also the host, making the host more susceptible to other infections (5). Antiseptics as treatment provides its own set of drawbacks, as there tends to be only a slight improvement and the issue of unnecessary host inflammation still remains $(5,220)$. This 
method suggests that using anti-inflammatory drugs, bone sparing agents, and pro-resolving agents may provide a new angle into treating oral diseases $(5,221,222)$. Another proposed method of treatment is photodynamic therapy, where long-wavelength visible light (red light) is used to activate photosensitizing agents that produce ROS response by the host, which proved to be more effective than treatment with antibiotics $(5,223)$.

Perhaps the most promising of the treatment options being considered are those that involve targeting the host (5). It is possible that host modulation therapy treatment provides a promising outcome for patients afflicted with periodontal disease, as the resolution of host inflammation will provide a more homeostatic environment in the oral cavity (5). As the shift in microbiota is vital for maintaining a state of health over disease in the oral cavity, the use of probiotic treatment remains a promising avenue that needs further research $(5,224)$. It has been suggested that development of active or passive immunizations, known to target gingipains, proteases, and peptide-binding sites, may prove beneficial in the treatment of disease, as there were promising pre-clinical studies performed on $P$. gingivalis $(1,225-227)$. A passive local monoclonal antibody treatment targeting $P$. gingivalis proteins showed prevention of recolonization in periodontal lesions up to 9 months after initial treatment $(1,225)$. Perhaps a promising therapeutic for these studies are the attenuation of inflammation generated by neutrophils in response to bacterial presence in the oral cavity. Studies performed with $P$. gingivalis showed that direct targeting of receptors chemerin receptor 23 (CHEMR23) and leukotriene B4 receptor 1 (BLT1), known to play a role neutrophil infiltration and cytokine production, resulted in resolution of inflammation and prevention of disease development (1).

Neutrophils are critical for providing the first response to invading microbes, in order to kill their target and allow for maintenance of host health; however, due to their production of toxic products, their resolution through apoptosis and macrophage clearance is just as important to ensuring host health rather than collateral host damage. Anti-neutrophil therapies have been 
proposed as treatment methods for periodontal disease, however, it is necessary for their antibacterial killing mechanisms to not be impaired, as this could exacerbate infection of the periodontium, among other sites (179). An ideal therapeutic candidate would be able to manipulate neutrophil surface receptors and impact signaling, without comprising killing mechanisms $(179,228)$. Current classes of inhibitors under investigation include: leukotriene B4 (LTB4) antagonists, chemokine receptor 2 (CXCR2) antagonists, long-acting b2-agonists and histone deacetylase (HDAC) activators $(179,229)$.

\section{Dissertation goals.}

The goals of this dissertation are to perform the first studies looking at the interaction of human neutrophils and periodontal pathogen, Filifactor alocis. In Chapter 2, we challenge neutrophils with $F$. alocis and analyze its effect on signaling pathway activation, degranulation and cell migration. In Chapter 3, we characterize the neutrophil oxidative-based killing mechanism employed against $F$. alocis and show how the bacterium evades this mechanism in

order to promote its own survival. In Chapter 4, we show that $F$. alocis fails to induce NETs, but is capable of manipulating neutrophils, impacting their ability to form NETs by known pharmacological and bacterial inducers. 


\section{CHAPTER 2}

\section{FILIFACTOR ALOCIS PROMOTES NEUTROPHIL DEGRANULATION AND CHEMOACTIC ACTIVITY}

Filifactor alocis is a recently recognized periodontal pathogen; however, little is known regarding its interactions with the immune system. As the first-responder phagocytic cells, neutrophils are recruited in large numbers to the periodontal pocket, where they play a crucial role in the innate defense of the periodontium. Thus, in order to colonize, successful periodontal pathogens must devise means to interfere with neutrophil chemotaxis and activation. In this study, we assessed major neutrophil functions, including degranulation and cell migration associated with the p38 mitogen-activated protein kinase (MAPK) signaling pathway upon challenge with $F$. alocis. Under conditions lacking a chemotactic gradient, $F$. alocis-challenged neutrophils had increased migration compared to uninfected cells, indicating that $F$. alocis increases chemokinesis in human neutrophils. In addition, neutrophil chemotaxis induced by interleukin-8 was significantly enhanced when cells were challenged with $F$. alocis compared to noninfected cells. Similar to live bacteria, heat-killed $F$. alocis induced both random and directed migration of human neutrophils. The interaction of F. alocis with Toll-like receptor 2 induced granule exocytosis along with a transient ERK1/2 and sustained p38 MAPK activation.

Moreover, F. alocis-induced secretory vesicle and specific granule exocytosis were p38 MAPK dependent. Blocking neutrophil degranulation with TAT-SNAP23 fusion protein significantly

\footnotetext{
${ }^{1}$ Armstrong CL, Miralda I, Neff AC, Tian S, Vashishta A, Perez L, Le J, Lamont RJ, Uriarte SM. 2016. Filifactor alocis promotes neutrophil degranulation and chemotactic activity. Infect Immun 84:3423-3433. doi:10.1128/IAI.00496-16
} 
reduced the chemotactic and random migration induced by $F$. alocis. Therefore, we propose that induction of random migration by $F$. alocis will prolong neutrophil traffic time in the gingival tissue, and subsequent degranulation will contribute to tissue damage. 
$\underline{\text { Introduction }}$

Periodontitis is a multifactorial chronic inflammatory disease, induced by a dysbiotic polymicrobial community of bacteria $(147,230)$. It is the sixth most common infectious disease worldwide, and over half the U.S. population will experience some form of periodontal disease (231). Additionally, accumulating epidemiological and mechanistic studies establish a causal association of periodontal disease and periodontal pathogens with serious systemic conditions, including, pneumonia, cardiovascular disease, preterm low birthweight delivery and some forms of cancer $(147,232,233)$.

Recent human oral microbiome studies reveal the presence of large numbers and diversity of fastidious and 'yet-to-be cultivated' taxons, many of which show a strong correlation with disease severity $(59,234)$. Nonetheless, the contribution to disease by these newly appreciated organisms has been overshadowed by the more readily cultivable species, and appreciation of their pathogenicity is just beginning to emerge $(235,236)$. Filifactor alocis is a slow-growing gram-positive anaerobe which in culture-independent studies is consistently found at increased frequency and in elevated numbers at periodontal disease sites compared to healthy sites $(2,44,54,57,58,60,61,63,64,234,237,238)$. In vivo, $F$. alocis is found in subgingival biofilms (70), and the organism positively correlates with other periodontal pathogens such as $P$. gingivalis forming a co-occurrence group that is enriched across different oral habitats (239). In vitro, the organism also participates in synergistic community formation with other common periodontal bacteria (63). Notably, F. alocis is relatively resistant to oxidative stress (60), can produce trypsin-like proteases (60), and can invade and induce the secretion of proinflammatory cytokines from gingival epithelial cells (44), properties which could contribute to pathogenicity in the periodontal pocket.

Neutrophils are the core phagocytic defenders of the periodontal pocket and are recruited in large numbers after adhesion to, and transmigration through, blood vessel walls (179). As a 
major component of the innate host response, neutrophils contribute to the maintenance of periodontal health by protecting the tissue against bacterial infection (198). Indeed, defects in neutrophil recruitment of function such as neutropenia, leukocyte adhesion deficiency (LAD), and Chediak-Higashi syndrome, strongly predispose to periodontitis (240). Directional movement, or chemotaxis, of neutrophils toward sites of injury and inflammation occurs by sensing and deciphering a chemoattractant gradient $(114,117-120)$. Neutrophils are able to respond to signals from both intermediary chemoattractants (such as interleukin (IL)-8), which are encountered upon travel to sites of infection and inflammation, and from end target cellular chemoattractants (such as formylated bacterial peptides like fMLF), operating at the site of infection $(118,120,123)$. A high concentration of chemoattractants is an indication to neutrophils that the cells had arrived at their final destination, and the process of phagocytosis and oxygen-independent and -dependent killing begins (118).

In the inflamed periodontal tissue, chemotactic factor such as IL-8, as well as bacterial derived products, AMLF, will be abundant and guide neutrophils from the blood vessels through the gingival tissue towards the periodontal pocket. During the cell migration process, neutrophil granule exocytosis will take place and contribute to gingival tissue damage. Given the importance of neutrophils in innate defense of the periodontium, successful periodontal pathogens, both individually and in the context of dysbiotic communities, have devised means to interfere with neutrophil chemotaxis and/or bacteria killing abilities $(179,241)$.

In this study, we show for the first time that $F$. alocis interaction with human neutrophils results in enhanced random migration and chemotaxis towards IL-8. In addition, F. alocis through TLR2 activation induced extracellular signal-regulated kinase (ERK) and p38 MAPK phosphorylation which preceded the enhanced random migration and stimulated neutrophil granule exocytosis. Moreover, F. alocis-mediated neutrophil migration was dependent on the bacteriainduced degranulation. 


\section{$\underline{\text { Results }}$}

F. alocis challenge of human neutrophils resulted in stimulated cell migration with and without directionality.

Neutrophil migration towards sites of infection is a key early event in the process of protection against pathogenic microorganisms and in periodontal disease, neutrophils are recruited in high numbers into the gingival tissues and crevicular fluid to control and combat the bacterial infection (242). However, neutrophils from chronic periodontitis patients show dysfunctional chemotactic function which increase the cell transit time in the gingival connective tissue promoting collateral tissue damage (243). Using a mouse chamber model, we recently showed that F. alocis infection resulted in a rapid neutrophil infiltration to the site of infection (61). To determine whether F. alocis interaction with human neutrophils can impact neutrophil migration, chemotaxis assays were performed using the transwell system. Assessed by light microscopic analysis and cell migration quantification, unstimulated cells showed minimal cell migration in the absence of a chemotactic source, while as expected, there was a significant neutrophil migration towards the potent chemotactic formylated peptide, fMLF, as expected (Fig. 2-1A). Interestingly, F. alocis-challenged neutrophils showed significant migration towards buffer alone compared to unstimulated cells (Fig. 2-1A), which indicates that the bacterial challenge increased random cell migration in the absence of a chemoattractant source. However, migration towards AMLF was similar in all the conditions regardless of bacteria challenge. Thus, we sought to determine if the random migration induced by $\mathrm{F}$. alocis would have no impact on directed migration independent of chemotactic source.

Since IL-8 is an important chemotactic factor involved in neutrophil recruitment to the site of periodontal infection (179), we examined the impact of $F$. alocis-challenge on IL-8-dependent neutrophil chemotaxis. Unlike what was observed with fMLF, the $F$. alocis-challenged cells showed a significant increase in chemotaxis towards IL-8 compared to unstimulated cells (Fig. 21B). Neutrophils can also be a source of IL-8 as they have pre-formed IL-8, which is stored in 
rapid mobilized vesicles (244), and can also synthesize the chemokine upon stimulation (245). To determine if the enhanced migration observed after $F$. alocis stimulation could be related to the release of the IL-8 from the stored pools, neutrophil supernatants were collected after 60 min of bacterial challenge and added to the lower chamber of the transwell system. As shown in Fig 21C, supernatant collected after 60 min of $F$. alocis challenge did not induce neutrophil migration, whereas both fMLF and IL-8 induced significant neutrophil chemotaxis. In addition, we were unable to detect IL-8 levels in the supernatant collected after 60 min of $F$. alocis challenge (data not shown), arguing against the possibility of an autocrine IL-8 effect induced by $F$. alocis responsible for the increased chemotaxis.

We next examined if bacterial viability is critical for inducing neutrophil motility by challenging cells with viable or heat-killed $F$. alocis. Both viable and heat-killed $F$. alocis challenged neutrophils displayed significantly enhanced random migration in the absence of a chemotactic source (Fig. 2-1D), no difference in directed cell migration toward fMLF compared to unchallenged control cells (Fig. 2-1E) and enhanced chemotaxis towards IL-8 (Fig 2-1F). Collectively these results indicate that $F$. alocis heat-stable cell wall components can stimulate random migration and can enhance the migration of neutrophils with directionality towards intermediary chemoattractants such as IL-8.

F. alocis interaction with human neutrophils induced granule exocytosis through TLR2 with ERK and $\mathrm{p} 38 \mathrm{MAPK}$ activation.

Components of gram-positive bacteria are usually recognized by Toll-like receptor (TLR) 2 which is expressed by neutrophils (246). Moreover, TLR2 agonists, such as $\mathrm{P}_{3} \mathrm{CSK}_{4}$, can induce neutrophil random migration by triggering extracellular signal-regulated kinase (ERK) and p38 mitogen-activated protein kinase (MAPK) signal transduction pathways (247). To examine the ability of $F$. alocis to activate the MAPK signaling pathways, phosphorylation of ERK1/2 and p38 MAPK was measured by immunoblot analysis. Figures 2-2 A and B show that $F$. alocis infection 
increased the levels of ERK1/2 and p38 MAPK phosphorylation in neutrophils with maximum activation reached at $15 \mathrm{~min}$ for ERK $1 / 2$ and $30 \mathrm{~min}$ for $\mathrm{p} 38$. By $60 \mathrm{~min}$ after $F$. alocis challenge, a decrease in both ERK1/2 and p38 MAPK phosphorylation was observed. To further characterize the dependence of $F$. alocis- induced MAPK signaling on TLR2 recognition, the bacteriumreceptor interaction was blocked by a TLR2 mAB. Figure 2-3 lane 3 shows that blocking TLR2 significantly inhibited $F$. alocis-induced phosphorylation of ERK1/2. A similar inhibitory trend, although not reaching statistical significance, was observed for p38 MAPK phosphorylation. These results indicate that $F$. alocis- induced ERK1/2 signaling pathway is TLR2 dependent.

In addition to its role in MAPK signaling pathways, TLR activation can also induce other neutrophil functions such as exocytosis of neutrophil granule, which has also been linked to chemotaxis $(246,248,249)$. The exocytosis of secretory vesicles and specific granules increases not only the number but also the diversity of the receptor repertoire on neutrophil plasma membrane and facilitates cell firm adhesion and extravasation from the blood to the tissue (250). Hence, to examine whether $F$. alocis-enhanced random migration in the absence of a chemotactic source could be linked to granule mobilization, the increases on the cell plasma membrane of secretory vesicles and specific granule markers were determined by flow cytometry. Stimulation of neutrophils for 30 min with $F$. alocis resulted in a significant secretory vesicle release similar to the exocytosis induced by fMLF (Fig. 2-4A). In addition, specific granule exocytosis, as measured by expression of $\mathrm{CD} 66 \mathrm{~b}$ on the plasma membrane, was significantly increased by $F$. alocis stimulation (Fig. 2-4B). Activation of p38 MAPK has been associated with cell migration and with TNF and LPS-induced neutrophil granule exocytosis (249). In order to test the involvement of p38 MAPK signaling in F. alocis-stimulated granule exocytosis, neutrophils were pretreated with the p38 inhibitor SB-203580 before bacterial challenge. Figures 2-4C and D shows that blocking p38 MAPK resulted in a significant decrease of secretory vesicles and specific granule exocytosis. To confirm that $F$. alocis- induced upregulation of CD35 and CD66b at the plasma membrane was 
accompanied by the release of granule content, the extracellular release of albumin and lactoferrin, respectively, was determined by ELISA. Figure 2-4E and $\mathrm{F}$ show that $F$. alocis induced significant release of both albumin and lactoferrin in a p38 MAPK-dependent manner. These results demonstrate that $F$. alocis challenge triggered secretory vesicles and specific granule exocytosis, and that the process was p38 MAPK dependent.

Chemotactic factors can induce release of azurophil granule components such as $\beta$ glucuronidase (251), and the contribution of lysosome exocytosis and fusion with the plasma membrane, through the regulation of Rab27a, to the promotion of cell migration has been established (252). Hence, exocytosis of azurophil granules upon F. alocis challenge was measured by both increase of plasma membrane expression of the granule marker CD63 by flow cytometry and the release of the granule component myeloperoxidase (MPO). Figure 2-5A and B show that F. alocis challenge did not induce azurophil granule exocytosis. Moreover, increasing the amount of bacteria per neutrophil from an MOI of 10 to 50 did not result in azurophil granule mobilization, measured both by upregulation of the granule marker (Fig. 2-5A) and the release of MPO (Fig. 2$5 B)$.

Our data thus far show that $F$. alocis interaction with human neutrophils results in TLR2mediated phosphorylation of ERK1/2 and p38 MAPK, although the latter may not be solely dependent on TLR2. Moreover, F. alocis induced the exocytosis of secretory vesicles and specific granules through activation of the p38 MAPK signaling pathway. Hence, we wanted to determine if upstream of the p38 MAPK signaling pathway, $F$. alocis interaction with TLR receptors could be the initial trigger that induces granule release. Figure 6A shows that blocking TLR2 with $\mathrm{mAB}$ resulted in a significant inhibition of $F$. alocis-induced secretory vesicle exocytosis, as measured by expression of CD35 on the plasma membrane; whereas TLR4 mAB had a minimal inhibitory effect. Similarly, F. alocis-induced specific granule exocytosis was inhibited to the same extent in anti-TLR2 treated cells (data not shown). To provide additional 
evidence for a lack of TLR4 activation by F. alocis, a pharmacologic inhibitor CLI-095, which blocks the intracellular domain of TLR4 (253), was used with lipoprotein-free LPS as a control. Figure 6B shows that, as expected, blocking TLR4 signaling resulted in a significant inhibition of LPS-induced secretory vesicle exocytosis. In contrast, and concordant with the anti-TLR4 data, chemical blocking TLR4 resulted in a minimal inhibition of $F$. alocis-induced secretory vesicle exocytosis. These results show that $F$. alocis stimulation of both secretory vesicles and specific granule exocytosis is TLR2 and p38 MAPK dependent.

Blocking neutrophil degranulation inhibits $F$. alocis-induced neutrophil cell migration.

Both p38 MAPK activation and neutrophil granule exocytosis play a key role in early stage neutrophil responses, such as diapedesis and chemotaxis $(249,252)$. Therefore, we sought to assess whether neutrophil granule exocytosis played a role in $F$. alocis-induced neutrophil migration in the presence or in the absence of a chemotactic source. Neutrophil degranulation can be blocked, without affecting neutrophil phagocytic ability and activation of p38 MAPK, by using the TATSNAP-23 fusion protein (146). First, we wanted to confirm that the TAT-SNAP-23 pre-treatment would block F. alocis- induced granule exocytosis. Figure 2-7 A and B shows that the TAT-SNAP23 fusion protein, as previously characterized (146), significantly blocked fMLF-stimulated secretory vesicles and specific granule exocytosis (Fig. 2-7A and B) and had no nonspecific effect when using a stimulation procedure, in this case with zymosan, that did not induce granule exocytosis. Upon verification that TAT-SNAP-23 was significantly blocking $F$. alocis-induced granule exocytosis, we tested the effect on cell migration. Figure $2-7 \mathrm{C}$ shows that treatment of unstimulated cells with the TAT-SNAP-23 fusion protein reduced the number of neutrophils crossing the membrane towards fMLF, which further emphasizes the role of granule exocytosis in neutrophil chemotaxis. On the contrary, when zymosan was used to stimulate neutrophils, the particulate stimuli induced chemotaxis toward IL-8, but pretreatment with the TAT-SNAP-23 fusion protein had no effect on zymosan-induced chemotaxis (Fig. 2-7D). However, as shown in Fig 2-7E, blocking neutrophil degranulation with TAT-SNAP-23 resulted in a significant inhibition 
of $F$. alocis- induced random migration in the absence of a chemotactic source. In addition, blocking granule release significantly reduced the ability of $F$. alocis-challenged neutrophils to migrate towards both chemotactic sources, fMLF and IL-8 (Fig 2-7E and F). These results demonstrate that when granule exocytosis is involved in cell migration, pre-treatment with the TAT-SNAP-23 fusion protein prevents exocytosis-mediated cell migration. Collectively, these data suggest that the enhanced migration of $F$. alocis-challenged neutrophils observed in the presence or in the absence of a chemotactic source is due in part to the p38 MAPK-dependent granule exocytosis induced by the bacteria challenge.

\section{Discussion}

Given the importance of neutrophils in innate defense of the periodontium, successful periodontal pathogens, both individually and in the context of dysbiotic communities, have devised means to interfere with neutrophil chemotaxis and killing $(179,241)$. Furthermore, congenital diseases, such as Leukocyte Adhesion Deficiency, that impair neutrophil chemotaxis result in severe periodontitis at early stage in life $(254,255)$. In the mouse subcutaneous chamber model of infection, the newly appreciated periodontal pathogen $F$. alocis elicits a local inflammatory response with extensive neutrophil recruitment, and spread to remote tissues, inducing lung edema with neutrophil recruitment, and causing acute kidney injury (61). However, very little is known about the pathogenic nature of $F$. alocis and its interaction with the innate immune system. In the present study, we showed that $F$. alocis interaction with human neutrophils, through TLR2 recognition, resulted in enhanced random and directed migration, and degranulation via activation of the p38 MAPK signaling pathway. Degranulation and sustained p38 MAPK activation induced by $F$. alocis were major contributors of the enhanced cell migration. Based on our data, a schematic model for neutrophil degranulation and enhanced migration in response to $F$. alocis challenge is proposed in Fig. 8. 
In the periodontal pocket, there is a continual influx of neutrophils that decipher and migrate through a chemotactic gradient. Our results showed that $F$. alocis interaction with human neutrophils resulted in a significant increase in random migration. Pre-exposure to F. alocis did not affect the ability of the cells to migrate with directionality towards fMLF; which is consistent with previous observations of human neutrophils challenged with either TLR 2/1 or TLR4 agonists such as $N$-palmitoyl-S-[2, 3-bis (palmitoyloxy)-(2RS)-propyl]-(R)-cysteinyl-seryl-(lysyl)(3)-lysine $\left(\mathrm{P}_{3} \mathrm{CSK}_{4}\right)$ or LPS respectively (247). In an inflamed tissue different neutrophil chemotactic products, such as IL-8, formylated peptides, and complement products such as C5a, are produced by different sources; however, neutrophils efficiently transit towards the end target by migrating in sequence from one chemotactic source to another (256). Fan and Malik showed that neutrophils activated by the TLR4 agonist, LPS, display enhance migration towards IL-8 by modulating CXCR2 expression and preventing receptor desensitization (257). We found here that $F$. alocis significantly enhanced neutrophil chemotaxis towards IL-8 through TLR2 activation. Hence, both TLR2 and TLR4 activation of neutrophils can result in enhanced migration towards IL-8. Another important signaling mechanism linked to neutrophil chemotaxis towards IL-8 or fMLF is differential activation of the two integrin molecules MAC-1 or LFA-1 (124). Activation of LFA1 or MAC-1 enhances neutrophil chemotaxis towards IL-8 or fMLF, respectively (124). Therefore, it is possible that $F$. alocis-enhanced chemotaxis towards IL-8 is due to LFA-1 activation. In addition, $F$. alocis-induced secretory vesicle exocytosis would increase the number and variety of receptors on the cell plasma membrane, making neutrophils more prone to mounting an enhanced response upon subsequent stimulation. Moreover, the F. alocis-induced release of specific granule content, which, among other proteins, includes members of the matrix metalloprotease family, such as collagenase and gelatinase, will contribute to tissue damage.

The MAP kinase family signaling components ERK and p38 MAPK play important roles in the regulation of fMLF-induced neutrophil migration $(119,122,258,259)$, whereas IL-8 
stimulation results in activation of the PI3K signaling pathway (260). Stimulation of neutrophils with TLR2 or TLR4 agonists signals through ERK and p38 MAPK to control random migration and chemotactic activity (247). Hence, the role of the different kinases in human neutrophil migration is dependent on the agonist. Our study showed that $F$. alocis triggered activation of both ERK and p38 MAPK but with temporal differences, with ERK activation, which occurred through TLR2, peaking at 15 min, whereas p38 MAPK showed a different phosphorylation pattern, increasing with time and peaking at $30 \mathrm{~min}$. Neutrophils migrate with directionality to sites of infection by following increasing concentrations of a chemoattractant, but when the chemoattractant concentrations are elevated, it is an indication that the cells reached their final destination, so a stop signal is triggered to prevent more migratory movement (261). The balance between ERK and p38 MAPK activation fine tunes neutrophil chemotaxis, as ERK regulates the stop signal and p38 MAPK promotes constant migration by suppression of the stop mechanism (261). Thus, the phosphorylation pattern of p38 MAPK, along with the transient phosphorylation of ERK induced by $F$. alocis, would lead to enhanced random migration and chemotaxis toward IL-8 as the result of the constant suppression of the stop signal by p38 MAPK, allowing sustained migration. In addition, fMLF-induced chemotaxis is regulated by ERK and p38 MAPK, and similarly, F. alocis challenge results in activation of both MAP kinases, which suggests that the oral pathogen induces signaling pathways similar to those of fMLF to stimulate neutrophil chemotaxis.

Upon stimulation, neutrophils will mobilize their granules which will either fuse with the cytoplasmic membrane or the phagosomal membrane, ultimately resulting in functional responses including exocytosis, extravasation, phagocytosis and elimination of various microorganisms (262264). Several neutrophil responses, including exocytosis, chemotaxis, respiratory burst activity and chemokine synthesis, are mediated through the p38 MAPK pathway (249). Activation of p38 MAPK signaling has been associated also with permitting neutrophils to sense and interpret the 
chemotactic sources, by controlling the surface expression of adhesion molecules like CD11b and CD66b, and chemoattractant receptors for fMLF and IL-8 (9). In this study, F. alocis induced secretory vesicle and specific granule exocytosis, which was mediated through TLR2 activation and dependent on p38 MAPK. Secretory vesicles are organelles that are easy to mobilize and are involved in augmenting the number of receptors and adhesion molecules like CD11b/CD18, which participate in the adhesion and transmigration process. F. alocis also induced a significant increase in the plasma membrane expression of CD66b, a specific granule marker used to evaluate granule exocytosis, and also involved in adhesion to fibronectin and E-selectin (265). When granule exocytosis was blocked by the use of TAT-SNAP-23 fusion protein (146), both random and directed migration induced by $F$. alocis challenge were impeded. It is plausible that $F$. alocisinduced secretory vesicle and specific granule exocytosis contributes to the enhanced chemotaxis towards IL- 8 by increasing the availability of CXCR2 receptors, as well as neutrophils adhesion capabilities by increase plasma membrane expression of CD66b. Moreover, we can speculate that in the context of periodontitis, besides the role of granule exocytosis on cell migration, the release of granule content to the extracellular space induced by $F$. alocis will contribute to tissue damage and disease progression.

Successful periodontal bacteria employ a variety of strategies to compromise neutrophil function. The major outer sheath protein (Msp) of Treponema denticola alters the balance of intracellular phosphoinositide, causing impairment of neutrophil directional migration towards fMLF and inhibition of downstream events leading to chemotactic responses (266). Msp does not form a pore in neutrophils but remains associated to the plasma membrane and triggers "outsidein" signaling that results in inhibition of PI3-kinase activity and an increase in the activity of the phosphatase PTEN. The MSP virulence factor favors neutrophil PTEN activity over PI3K, resulting in a decrease on the amount of the phosphoinositide PIP3, which compromises actin dynamics, preventing the cell from having proper directed chemotaxis (266). The keystone 
periodontal pathogen, $P$. gingivalis, generates a local and transient chemokine paralysis by antagonizing the synthesis and release of IL-8 from gingival epithelial cells (267). The transient suppression of neutrophil recruitment to the gingival tissue facilitates the colonization of the tissue by $P$. gingivalis and other oral bacteria (268). Interaction between $F$. alocis and gingival epithelial cells results in release of IL-8 (44), and the current study shows that when $F$. alocis interacts with human neutrophils, there is no significant difference in cell migration towards fMLF compared to unstimulated cells, but significantly enhanced migration towards IL-8. Hence $F$. alocis may function in obstructing the neutrophil from distinguishing between intermediary (IL-8) and endtarget chemoattractants (fMLF). As it is necessary for neutrophils to eventually migrate towards end-target chemoattractants in order to reach sites of infection, $F$. alocis manipulation of neutrophils could lead to defective deciphering abilities between chemoattractant sources, leading to constant migration and cell activation which could contribute to dysregulated and sustained inflammation and to tissue damage.

In conclusion, we showed that $F$. alocis induces a significant random and directed migration of human neutrophils towards IL-8. Activation of TLR2 by F. alocis incites a transient ERK 1/2 activation secretory vesicle and a sustained p38 MAPK activation, which results in exocytosis of secretory vesicles and specific granules. Ultimately, the p38 MAPK-dependent degranulation was responsible for $F$. alocis-enhanced neutrophil migration, which may contribute to dysbiotic host responses and promote tissue damage by activated neutrophils (Fig. 8).

\section{Material and Methods}

Neutrophil isolation.

Neutrophils were isolated from blood of healthy donors using plasma-Percoll gradients as previously described (146), and in accordance with the guidelines approved by the Institutional Review Board of the University of Louisville. Microscopic evaluation of the isolated cells showed 
that $>95 \%$ of the cells were neutrophils. Trypan blue exclusion indicated that $>97 \%$ of cells were viable.

\section{Bacterial strains and growth conditions.}

F. alocis ATCC 38596 was cultured in brain heart infusion (BHI) broth supplemented with L-cysteine $(0.1 \%)$ and arginine $(20 \%)$ for 7 days anaerobically at $37^{\circ} \mathrm{C}$ as previously described [27, 42]. Heat killed $F$. alocis was generated by incubation at $90{ }^{\circ} \mathrm{C}$ for $60 \mathrm{~min}$.

\section{TAT-SNAP-23 fusion protein.}

Fusion proteins were created as previously described (146). E. coli BL21-AI cells (Invitrogen) were transformed to overexpress the recombinant TAT fusion proteins. Purification of TAT-SNAP-23 was performed by sonication and lysis of the bacterial pellet with a denaturing buffer (7 M urea, $0.5 \mathrm{M} \mathrm{NaCl}, 50 \mathrm{mM} \mathrm{NaPO}_{4}$ [pH 8], $20 \mathrm{mM}$ imidazole), followed by protein separation from the supernatant by Ni-NTA beads (Invitrogen). Protein eluted from the beads was dialyzed against $10 \%$ glycerol, $0.01 \%$ Triton $\mathrm{X}-100$ in PBS, $\mathrm{pH} 7.4$, and stored at $-80{ }^{\circ} \mathrm{C}$ until use. Neutrophil chemotaxis.

Freshly isolated neutrophils $\left(4 \times 10^{6}\right.$ cells $\left./ \mathrm{mL}\right)$ were unstimulated, or challenged with $F$. alocis (multiplicity of infection (MOI) 10), or pre-treated with TAT-SNAP23 (0.9 $\mu \mathrm{g} / \mathrm{mL}, 10 \mathrm{~min})$ followed by $F$. alocis challenge at $37^{\circ} \mathrm{C}$ for $30 \mathrm{~min}$. After appropriate treatment, $100 \mu \mathrm{l}$ of cell suspension were added to the upper chamber of the transwell inserts contained in 24 well plates (VWR, Corning, NY). Chemotaxis was initiated by adding $600 \mu \mathrm{L}$ of chemoattractants into the lower chamber. The chemoattractants used were fMLF (10 $\mu \mathrm{M}$, Sigma), IL-8 (100 ng/mL, Sigma), along with supernatants collected from unstimulated or F. alocis-challenged neutrophils (MOI 10, 1-4-20 h). After $30 \mathrm{~min}$, the transwell membranes were stained with a HEMA 3 stain set kit following the manufacturer's instructions (Thermo Fisher Scientific,). Chemotaxis was assessed by light microscopic (VWR Compound Trinocular Microscope) examination (magnification x100) 
of the underside of the membrane. The average number of cells from a total of 10 fields was determined and data were normalized by the area of membrane circle and field of view.

\section{ERK1/2 and p38 MAPK Kinase phosphorylation.}

Phosphorylation of ERK1/2 and p38 MAPK was determined as previously described (146, 269). In brief, neutrophils $\left(1 \times 10^{7}\right.$ cells $\left./ \mathrm{mL}\right)$ were unstimulated, stimulated with fMLF $(10 \mu \mathrm{M}, 1$ $\min$ ), or F. alocis (MOI 10, for 5-15-30-60 min), or for some experiments cells were pre-treated with anti-TLR2 antibody (50 $\mathrm{g} / \mathrm{ml}$, Biolegend, clone TL2.1), or isotype control IgG2akappa (50 $\mu \mathrm{g} / \mathrm{ml}$, Biolegend clone MOPC-173) followed by F. alocis (MOI 10, $15 \mathrm{~min}$ ). After the different experimental conditions, cells were centrifuged at $2500 \mathrm{~g}$ for $20 \mathrm{~s}$ and lysed in ice-cold lysis buffer [20 mM Tris- $\mathrm{HCl}$ (pH 7.5), $150 \mathrm{mM} \mathrm{NaCl}, 1 \%$ (vol/vol) Triton X-100, $0.5 \%$ (vol/vol) Nonidet P40, $20 \mathrm{mM} \mathrm{NaF}, 20 \mathrm{mM} \mathrm{NaVO}$, 1 mM EDTA, 1 mM EGTA, 5 mM PMSF, $21 \mu \mathrm{g} / \mathrm{mL}$ aprotinin, and $5 \mu \mathrm{g} / \mathrm{mL}$ leupeptin]. Cell lysates were separated by $4-12 \%$ gradient SDS-PAGE, and immunoblotted with antibodies to phospho ERK1/2, total ERK1/2, phospho-p38 MAPK, or total p38 MAPK (Cell Signaling) all at 1:1000. The appropriate secondary antibodies were used at 1:5000 (Santa Cruz). The ECL system (Amersham Pharmacia Biotech) was used to visualize antigen-antibody reactions. Densitometric values of each band was calculated using ImageJ software.

Neutrophil granule exocytosis.

Neutrophils $\left(4 \times 10^{6}\right.$ cell $\left./ \mathrm{mL}\right)$ were incubated with buffer (basal), or with $F$. alocis at MOI 10 for $30 \mathrm{~min}$. Inhibitors, pre-treated with the cells for $30 \mathrm{~min}$, were SB-203580 (3 $\mu \mathrm{M})$, antiTLR2 antibody (50 $\mu \mathrm{g} / \mathrm{ml}$, Biolegend, clone TL2.1), anti-TLR4 antibody (50 $\mu \mathrm{g} / \mathrm{ml}$, Biolegend, clone HTA125), and isotype control IgG2akappa (50 $\mathrm{g} / \mathrm{ml}$, Biolegend clone MOPC-173). For the role of TLR4 in F. alocis induced exocytosis, neutrophils were unstimulated, or stimulated with ultra-pure Lipopolysaccharide (LPS; $100 \mathrm{ng} / \mathrm{ml}, 60 \mathrm{~min}$ ) (ENZO Life Sciences), or pre-treated with a TLR4 signaling inhibitor CLI-095 (1 $\mu \mathrm{g} / \mathrm{ml}, 15 \mathrm{~min}$ ) (Invivogen) followed by either LPS (100 ng/ml, $60 \mathrm{~min}$ ) or F. alocis (MOI 10, $30 \mathrm{~min}$ ). Exocytosis of secretory vesicles and specific 
granules was determined by measuring the increase in plasma membrane expression of PEconjugated anti-human CD35 (Biolegend, clone E11) and FITC-conjugated anti human CD66b (Biolegend, clone G10F5). Antibody reactivity was measured with a BD FACSCalibur Flow Cytometer (Becton Dickinson).

Statistical analysis.

For all the experimental conditions tested in this study, the statistical analysis used was a one-way ANOVA with the Tukey-Kramer multiple-comparison test (GraphPad Prism software, San

Diego, CA, USA). Differences were considered statistically significant at the level of $\mathrm{P}<0.05$. 


\section{Figures and Figure Legends}
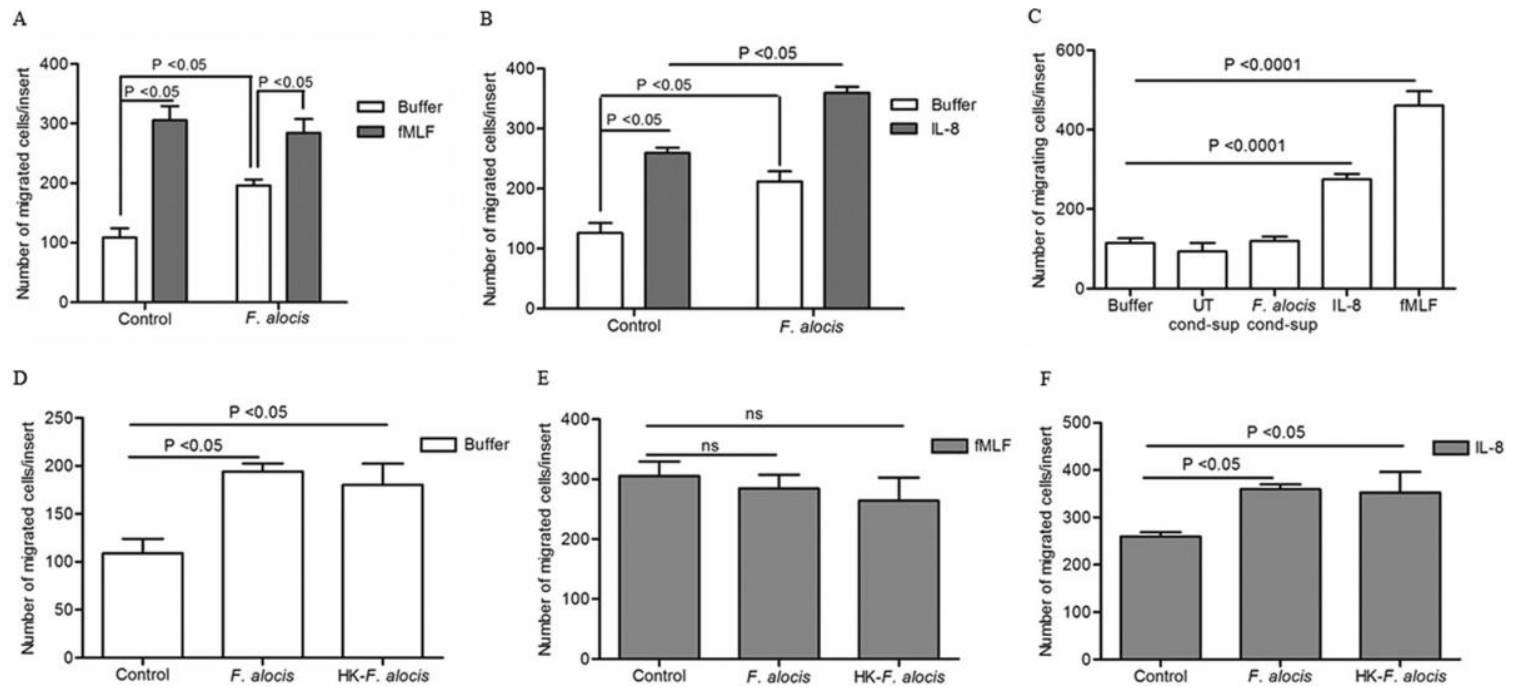

Figure 2-1. Effect of $F$. alocis stimulation on neutrophil chemotaxis.

Neutrophils were left unchallenged (control), challenged with F. alocis (30 min), or challenged with heat-killed F. alocis (HK-F. alocis; 30 min). (A to F) Following the bacterial challenge, cells were placed in the upper chamber of the transwell system, and after $30 \mathrm{~min}$ of incubation, the membrane was stained with a HEMA 3 stain set kit. Chemotaxis was assessed by light microscopic examination (magnification, 100). (A) Buffer or fMLF (100 nM) was placed in the lower well. Data are expressed as mean numbers standard errors of the mean (SEM) of migrated cells/insert from 9 independent experiments. (B) Buffer or IL-8 (100 ng/ml) was placed in the lower well. Data are expressed as mean numbers SEM of migrated cells/insert from 5 independent experiments. (C) Unstimulated cells were placed in the upper chamber of the transwell plate, and buffer, conditioned supernatant collected from unstimulated cells (UT-cond-sup), or conditioned supernatant collected after $60 \mathrm{~min}$ of stimulation with $F$. alocis (F. alocis-cond-sup), IL-8 (100 ng/ml), or fMLF (100 nM) was placed in the lower well. Data are means SEM from 6 independent experiments. (D to F) Buffer (D), fMLF (E), or IL-8 (F) was placed in the lower well. Data are expressed as mean numbers SEM of migrated cells/insert from 5 independent experiments. 

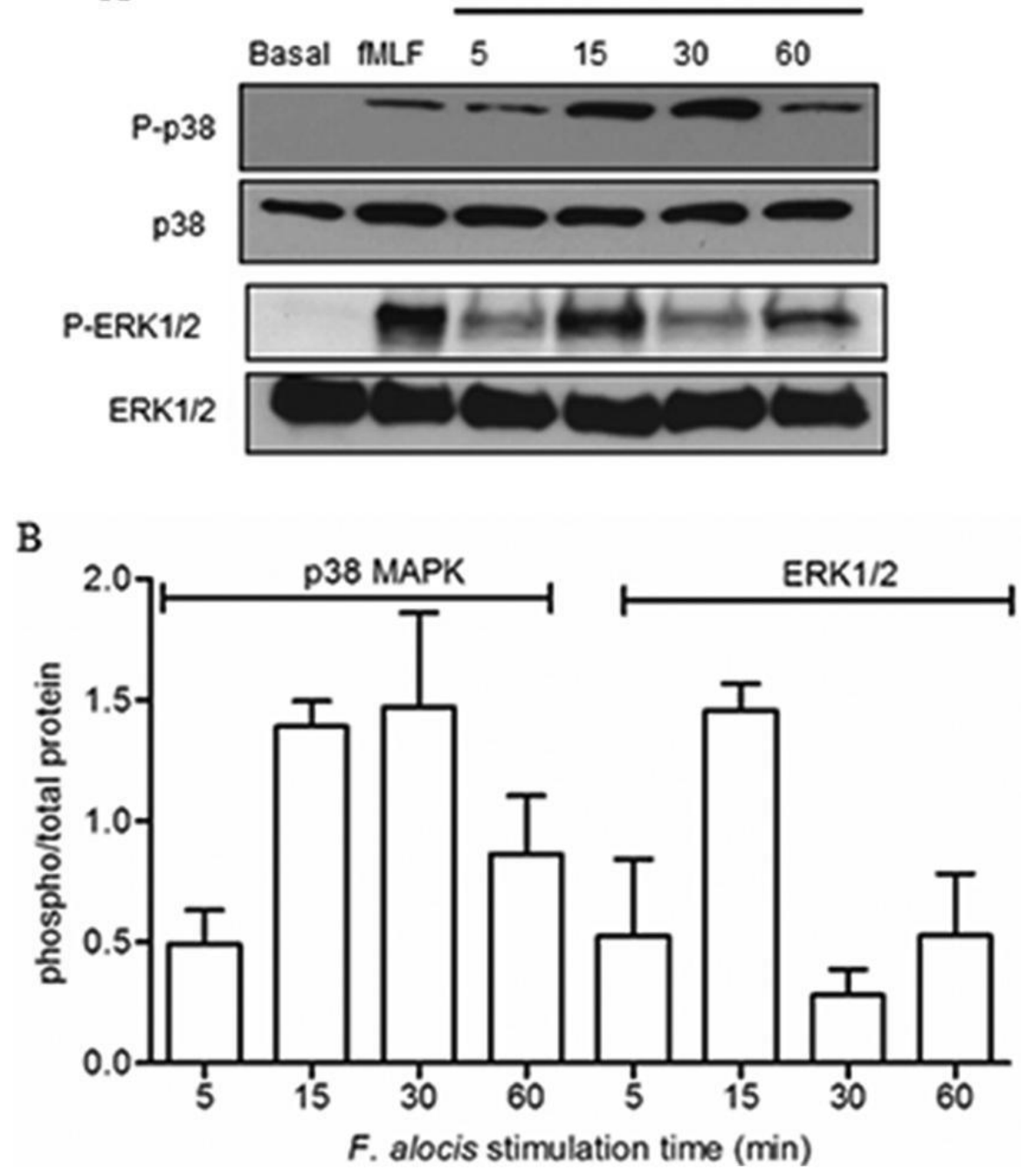

Figure 2-2. F . alocis-induced ERK1/2 and p38 MAPK activation in human neutrophils.

Neutrophils were unchallenged (basal), stimulated with fMLF (300 nM, 1 min), or challenged with F. alocis for the indicated times. Cells were lysed, and proteins were separated by SDS-PAGE and immunoblotted for phospho-p38 (P-p38) or phospho-ERK1/2 (P-ERK1/2). Blots were stripped and reblot-ted for total p38 (p38) or total ERK1/2 (ERK1/2), respectively. (A) Represen-tative immunoblot of 5 independent experiments. (B) Densitometric analysis of the 5 immunoblots for Pp38 or P-ERK1/2 normalized to the total amount of p38 or ERK1/2, respectively. Data are expressed as the mean ratio SEM of phosphorylated to total kinase. 

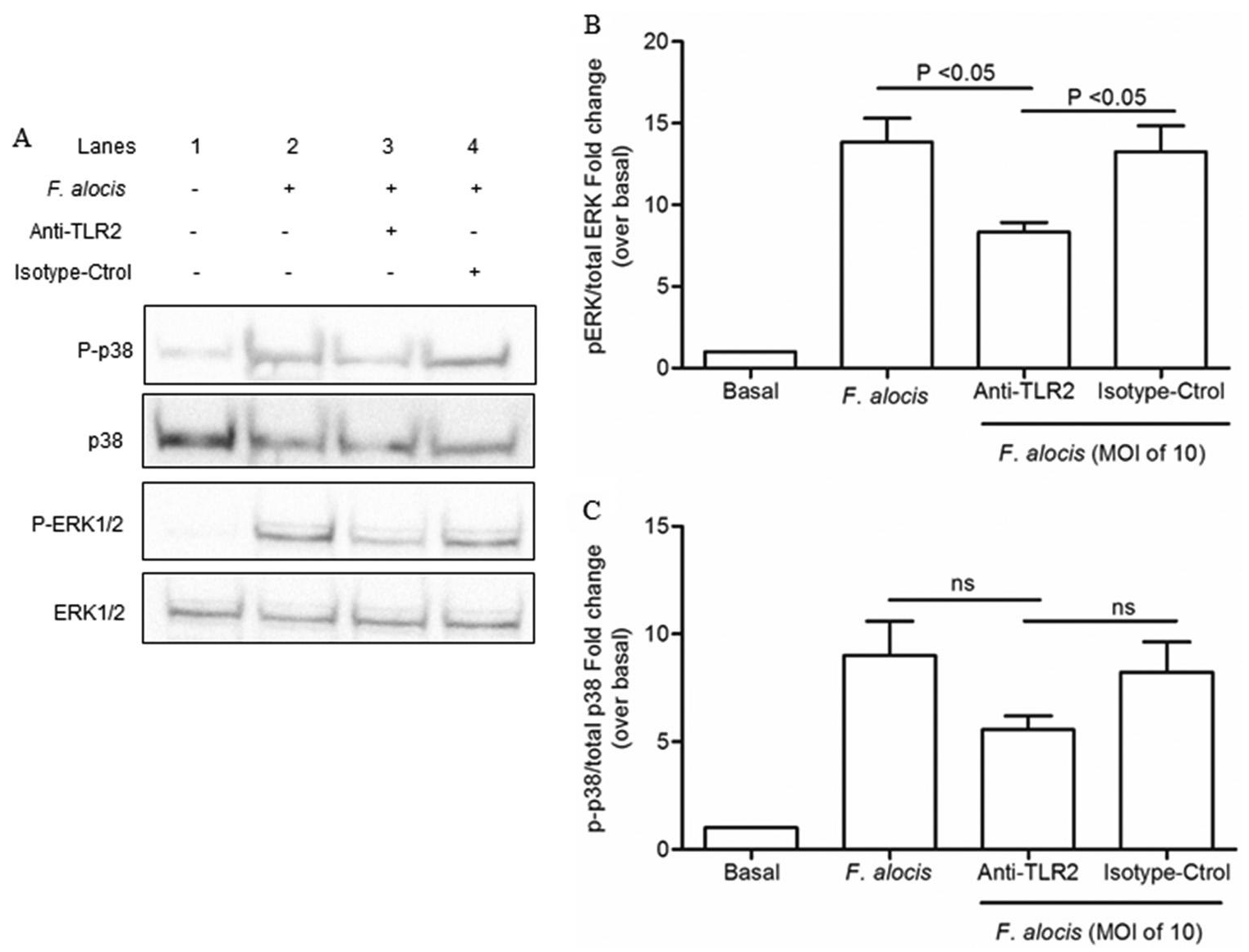

Figure 2-3. TLR2 activation is involved in $F$. alocis-induced phosphorylation of both ERK1/2 and p38 MAPK.

Neutrophils were unchallenged (basal), challenged with $F$. alocis (MOI of 10, $15 \mathrm{~min}$ ), or pretreated with either anti-TLR2 MAb or isotype control (isotype-Ctrol), followed by F. alocis challenge. Cells were lysed and proteins separated by SDS-PAGE and immunoblotted for phosphop38 (P-p38) or phospho-ERK1/2 (P-ERK1/2). Blots were stripped and reblotted for total p38 (p38) or total ERK1/2 (ERK1/2), respectively. (A) Representative immunoblot of 4 independent experiments. (B) Densitometric analysis of the 4 immunoblots for P-ERK1/2/total ERK1/2. (C) Densitometric analysis of the 4 immunoblots for P-p38 MAPK/total p38 MAPK. Data are expressed as mean fold changes SEM over the basal level of the phosphorylated/total kinase ratio. 

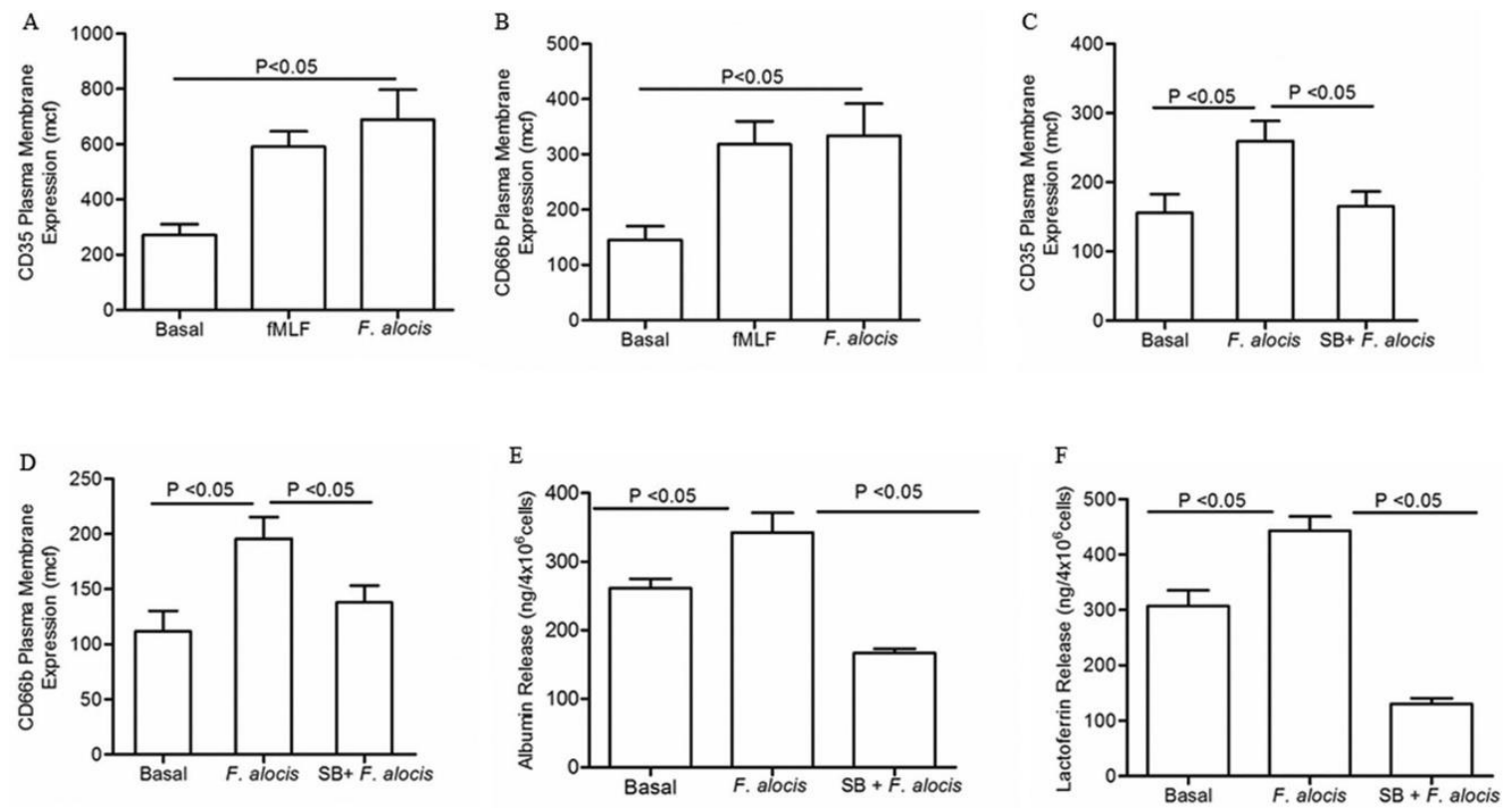

Figure 2-4. F. alocis stimulation of secretory vesicle and specific granule exocytosis is p38 MAPK dependent.

Neutrophils were left unchallenged (basal), challenged with fMLF (300 nM, 5 min), challenged with $F$. alocis (MOI of 10, $30 \mathrm{~min}$ ), or pretreated for $30 \mathrm{~min}$ with SB203580 followed by $F$. alocis challenge (SB F. alocis). (A to D) Secretory vesicle and specific granule exocytosis were determined by the increase in plasma membrane expression of the CD35 or CD66b marker, respectively, by flow cytometry. Data are expressed as the mean channel of fluorescence (mcf) SEM from 5 independent experiments. (E and F) Supernatants from all of the different experimental conditions were collected, and the release of albumin or lactoferrin to determine secretory vesicle or specific granule exocytosis, respectively, was measured by ELISA. Data from albumin or lactoferrin release are expressed as means SEM in ng/4 $10^{6}$ cells from 5 independent experiments for albumin and 6 independent experiments for lactoferrin. 

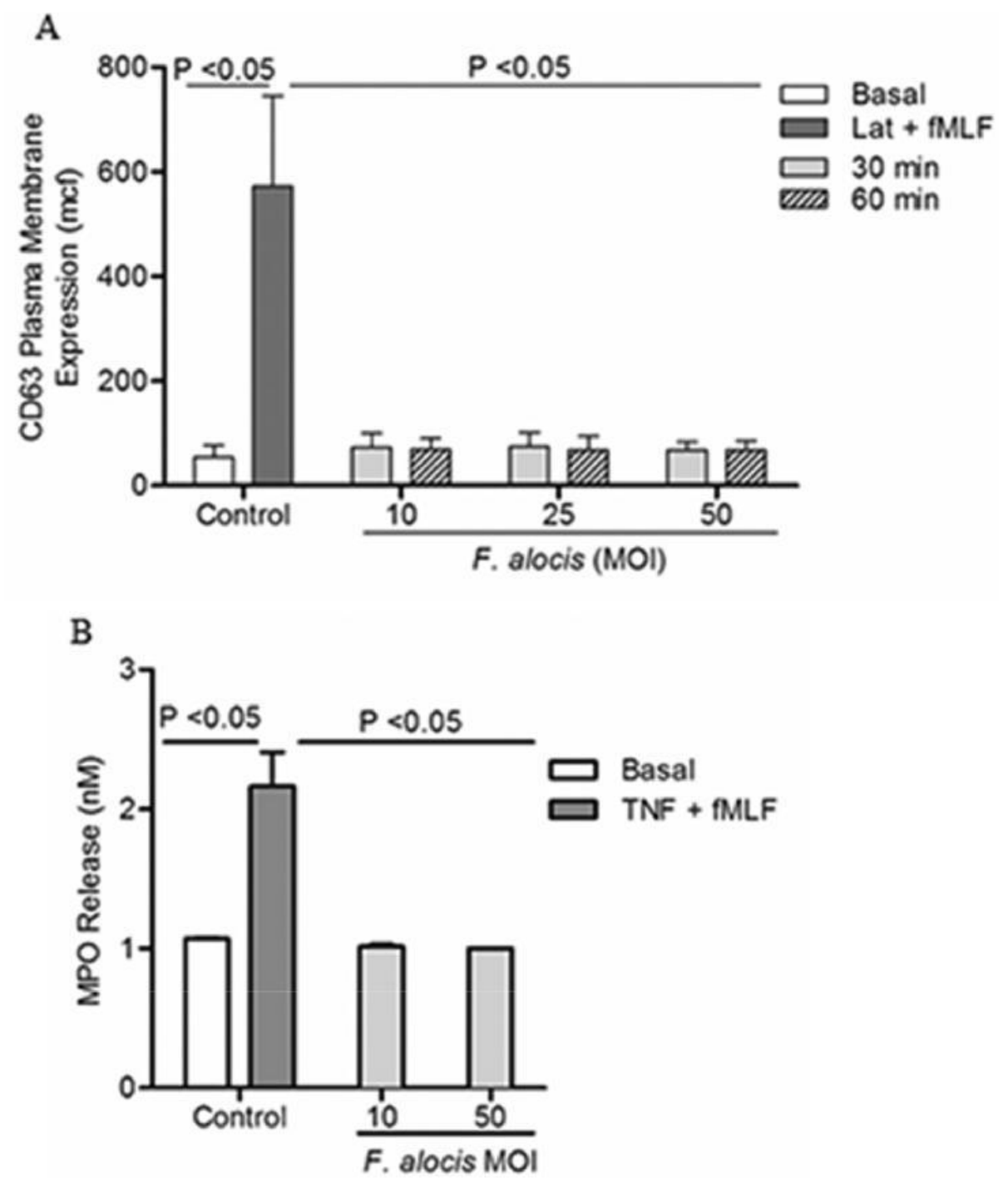

Figure 2-5. F. alocis challenge does not induce azurophil granule exocytosis.

Neutrophils were left unchallenged (basal), were pretreated with latrunculin-A (1 M, $30 \mathrm{~min})$ followed by fMLF stimulation (Lat fMLF, $300 \mathrm{nM}, 5 \mathrm{~min}$ ), challenged with TNF ( $2 \mathrm{ng} / \mathrm{ml}, 10 \mathrm{~min}$ ) followed by fMLF stimulation (TNF fMLF, $300 \mathrm{nM}, 5 \mathrm{~min}$ ), or challenged with $F$. alocis (MOI of 10, 25, and 50 for 30 or $60 \mathrm{~min}$ ). (A) Azurophil granule exocytosis was determined by the increase in plasma mem-brane expression of CD63 by flow cytometry. Data are expressed as mean mcf SEM from 3 independent experiments. (B) Extracellular release of myeloperoxi-dase (MPO), to determine azurophil granule exocytosis, was measured as described in Materials and Methods. Data from MPO release are expressed as means SEM in nM from 3 independent experiments. 

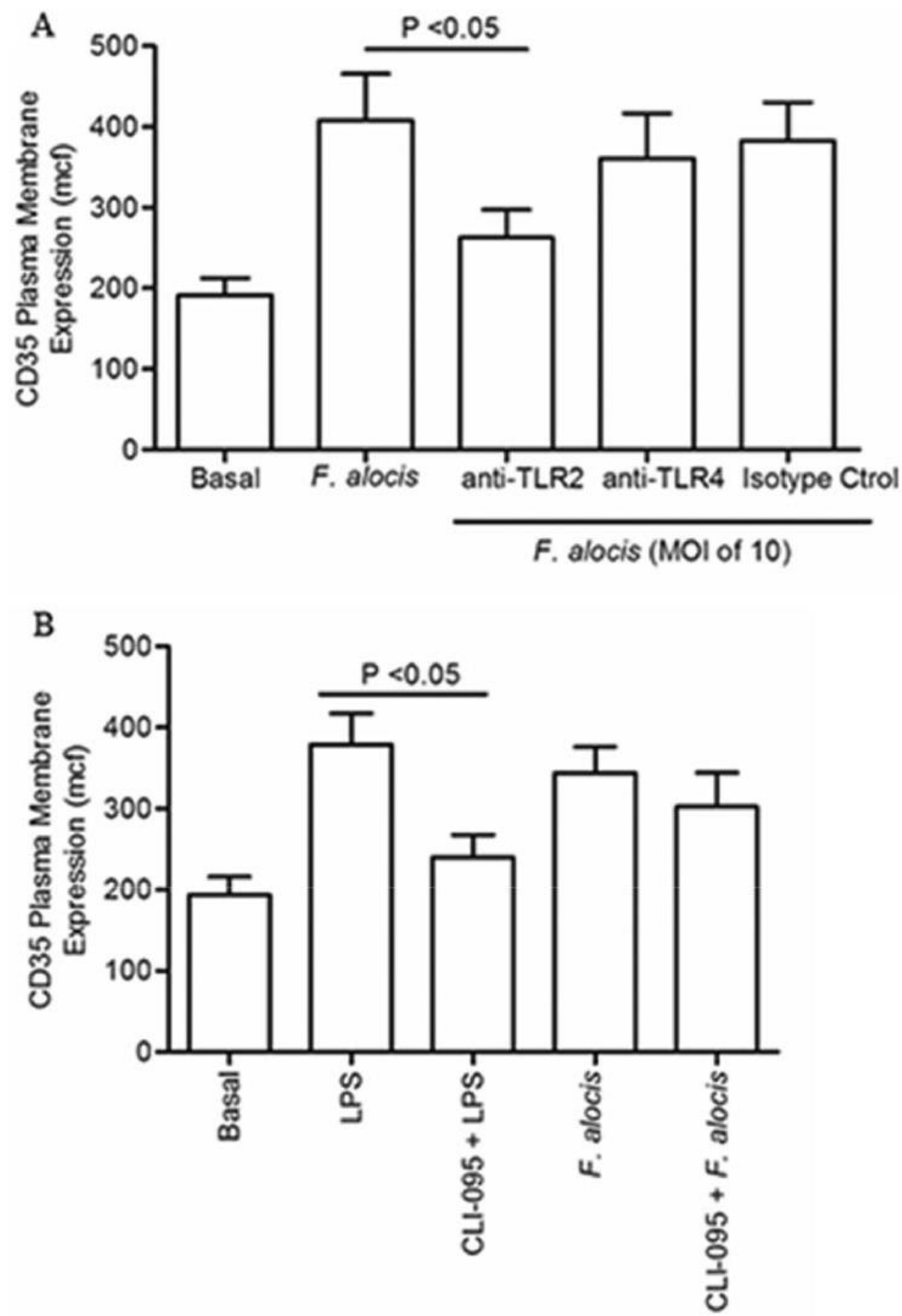

Figure 2-6. F. alocis interaction with TLR2 triggered secretory vesicle exocytosis.

(A) Neutrophils were left unchallenged (basal), challenged with F. alocis (M0I of 10, $30 \mathrm{~min}$ ), or pretreated for 30 min with either anti-TLR2 MAb, anti-TLR4 MAb, or isotype control (isotype ctrol) followed by $F$. alocis challenge. (B) Neutrophils were left unchallenged (basal), challenged with LPS (100 ng/ml, $60 \mathrm{~min}$ ), pretreated for $15 \mathrm{~min}$ with CLI-095 followed by LPS challenge, challenged with $F$. alocis, or pretreated with CLI-095 followed by $F$. alocis challenge. In both panels, secretory vesicle exocytosis was determined by the increase in plasma membrane 
expression of the CD35 marker by flow cytometry. Data are expressed as mean mcf SEM from 5 independent experiments. 

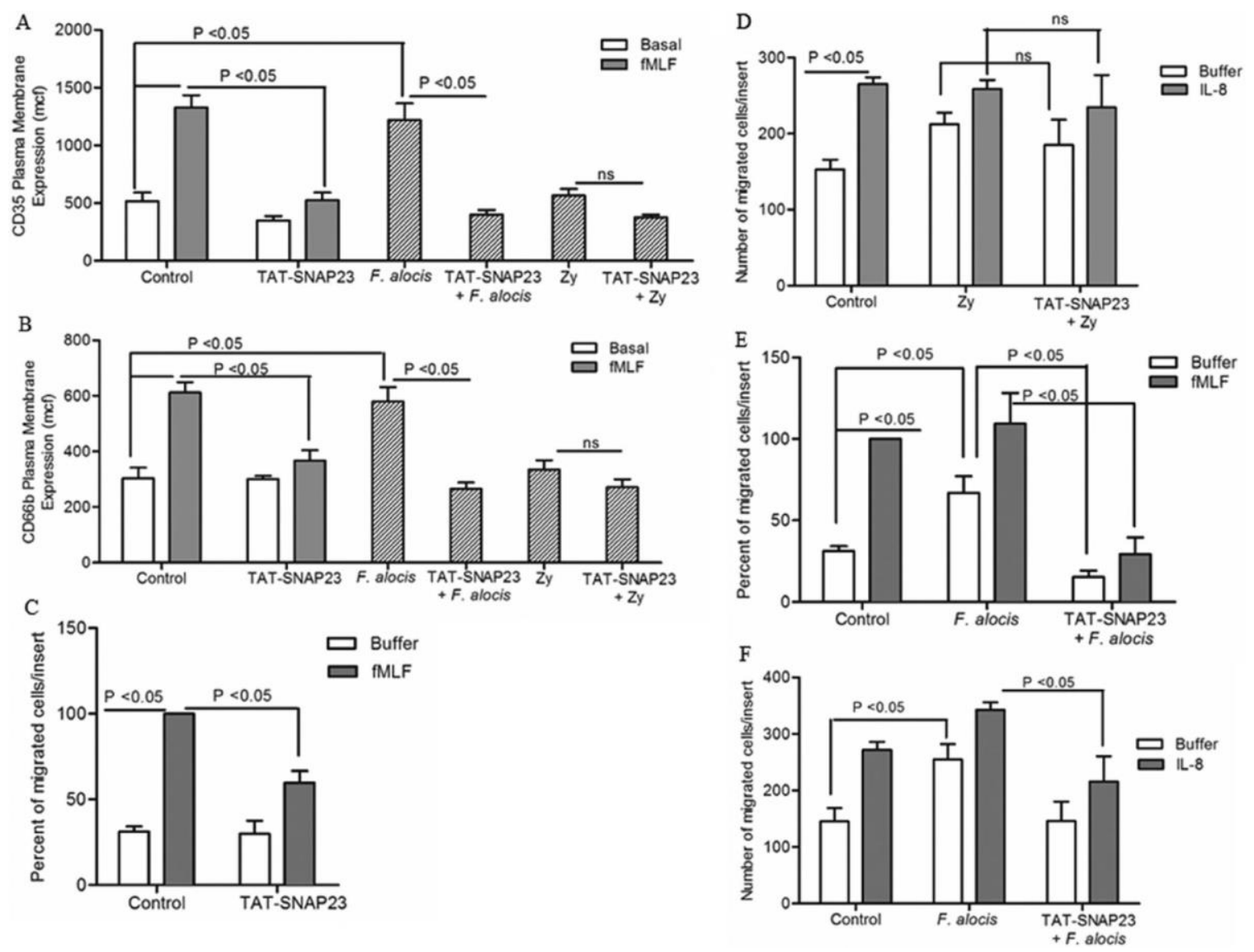

Figure 2-7. Blocking neutrophil granule exocytosis inhibits $F$. alocis-induced random and directed migration.

Neutrophils were left unchallenged (control), stimulated with fMLF (300 nM, 5 min), treated with TAT-SNAP23 (10 min), pretreated with TAT-SNAP23 followed by fMLF stimulation, challenged with $F$. alocis (30 min), challenged with zymosan (Zy; $30 \mathrm{~min}$ ), pretreated with TATSNAP23 (10 $\mathrm{min}$ ) followed by $F$. alocis challenge (TAT-SNAP23 F. alocis), or pretreated with TAT-SNAP23 followed by zymosan challenge (TAT-SNAP23 Zy). (A and B) Secretory vesicle and specific granule exocytosis were determined by the increase in plasma membrane expression of the CD35 or CD66b marker, respectively, by flow cytometry. Data are expressed as mean mcf SEM from 5 independent experiments. ( $\mathrm{C}$ to $\mathrm{F}$ ) Following cell stimulation or bacterial challenge, cells were placed in the upper chamber of the transwell system. After $30 \mathrm{~min}$ of incubation, the membrane was stained with a HEMA 3 stain set kit. Chemotaxis was assessed by light 
microscopic examination (magnification, 100). (C and E) Buffer or fMLF (100 nM) was placed in the lower well. Data are means SEM from 5 independent experiments. (D and F) Buffer or IL-8 $(100 \mathrm{ng} / \mathrm{ml})$ was placed in the lower well. Data are expressed as mean (SEM) number of migrated cells/insert from 5 independent experiments (D) and from 7 independent experiments (F). 


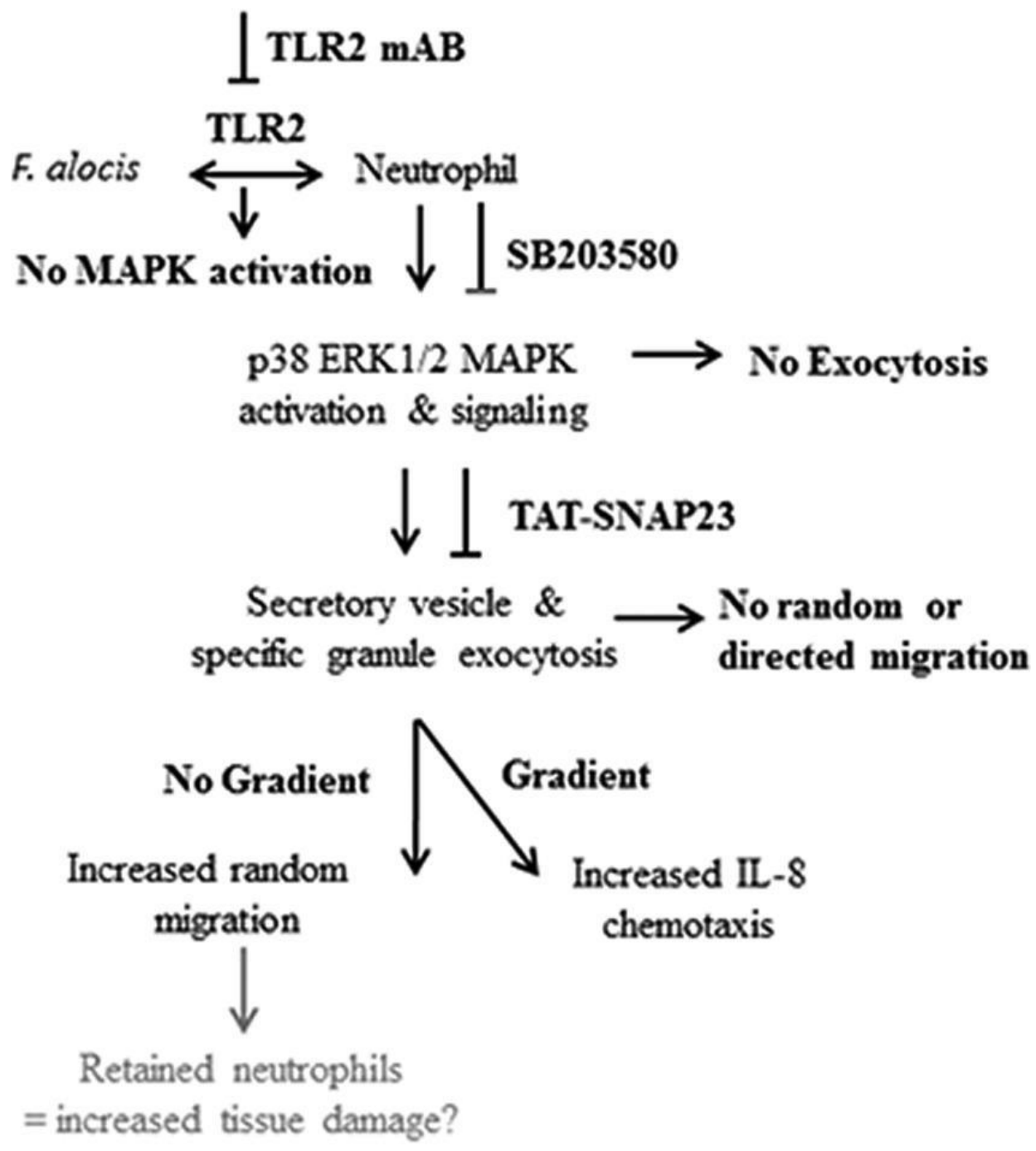

Figure 2-8. Schematic representation of $F$. alocis-induced neutrophil granule exocytosis, random and directed migration.

F. alocis binding to TLR2 on the neutrophil plasma membrane induces phosphorylation of ERK1/2 and p38 MAPK. Activation of p38 MAPK promotes the exocytosis of secretory vesicles and specific granules, which contribute to $F$. alocis-induced random and directed migration. $F$. alocis-induced neutrophil granule exocytosis, enhanced random migration and chemotaxis toward IL-8, could retain these activated professional phagocytes in the gingival tissue and increase tissue damage. 


\section{CHAPTER 3}

\section{F. ALOCIS EVADES NEUTROPHIL OXYGEN-DEPENDEN KILLING MECHANISMS}

\section{$\underline{\text { Introduction }}$}

Neutrophils are the prominent immune cell in circulation and known as the earliest responders of the innate immune system (270). Highlighting the importance of neutrophils is the fact that in patients with decreased numbers of circulating neutrophils, there is an enhanced risk for morbidity and mortality from infection; additionally, patients with a neutrophil genetic disorder often encounter frequent and severe infections following microbial challenge (270-272).

Following receptor-mediated phagocytosis of microbes, neutrophils are activated and utilize both oxygen-dependent and oxygen-independent killing mechanisms to kill their target $(143,273-275)$. The focus of our studies is on the oxygen-dependent respiratory burst response employed by human neutrophils in response to F. alocis challenge. Once the target is effectively internalized into the phagosome, a maturation process occurs where neutrophil granules fuse with the phagosome and release their antimicrobial compounds to cause an inhospitable environment (72). Additionally, granules can fuse with the plasma membrane, which results in the release of antimicrobial components into the extracellular space. Along with granule fusion to the phagosome or to the plasma membrane is the requirement of the activation of the NADPH oxidase complex at these sites, both processes occurring simultaneously (72). The NADPH oxidase complex can only become activated once its cytosolic components (p47phox,$p 67^{\text {phox }}$, $\left.\mathrm{p} 40^{\text {phox }}, \mathrm{Rac}\right)$ translocate from the cytosol and bind membrane components of the complex $\left(\mathrm{gp} 91^{\text {phox }}, \mathrm{p} 22^{\text {phox }}\right)$. The necessity for a functional NADPH oxidase is demonstrated clearly in CGD patients, a genetic disorder, which results in mutations in components of the complex 
and renders an inactive oxidase and leads to severe and recurrent bacterial and fungal infection (276-280).

After efficient NADPH oxidase assembly and activation and granule fusion, neutrophils will mount the oxidative respiratory burst response with generation of reactive oxygen species (ROS), which ultimately are responsible for killing targeted microbes, in the phagosome and in the extracellular space. It is believed that the degranulation and ROS production may function in concert and potentiate one another, leading to a highly efficient killing process employed by neutrophils (73).

The respiratory burst response by neutrophils is named so due to the rapid increase in oxygen consumption by activated neutrophils and results from a step-wise production of superoxide from the NADPH oxidase complex which dismutates and yields $\mathrm{H}_{2} \mathrm{O}_{2}$, and in the presence of MPO and chloride, the potent $\mathrm{HOCl}$ is produced $(143,270,281)$. The timing of superoxide production and whether this occurs at the plasma membrane or the phagosome depends on the type of stimulus encountered $(282,283)$. When neutrophils encounter soluble stimuli, like bacterial-derived peptide N-formyl-L-methionyl-L-leucyl-L-phenylalanine (fMLF), recognized by formyl peptide receptors (FPRs), the oxidase complex will assemble at the plasma membrane, targeting superoxide production in the extracellular space that usually lasts less than 5 $\min .(282,283)$. However, in the context of bacterial induction of the respiratory burst response, the NADPH oxidase complex assembly will be targeted for the phagosome, in order to ensure superoxide will be produced inside the phagosomal compartment containing the bacteria (127). Targeting ROS production to the phagosomal compartment is an effective strategy for neutrophils, as they will attempt to eliminate the bacteria without causing unnecessary host tissue damage, as can occur when superoxide is produced and released in the extracellular space. Is has been determined that the majority of intracellular pathogens are effectively controlled and eliminated through the respiratory burst response, as the response occurs rapidly (30-60 min postinfection) and neutrophils produce large amounts of highly potent ROS, like $\mathrm{HOCl}$ (143). 
Neutrophil priming is a pre-activation stage, where neutrophils are not fully active, but will become activated upon encountering a secondary stimulus (190). Once neutrophils are primed, they will induce an enhanced response to a secondary stimulus (284). Priming agents, administered both in vitro (e.g. TNF- $\alpha$, fMLF) and in vivo (e.g. GM-CSF, LPS, IL-8) turn on activation pathways without starting the activation process and they function to enhance fMLFinduced ROS production (190). In the primed state, the NADPH oxidase begins to undergo a preactivation process which includes protein redistribution, partial phosphorylation of the cytosolic component, $\mathrm{p} 47^{\text {phox }}$, as well as conformational changes; the activated state requires assembly of the complex (190). As observed in studies with LPS treatment of neutrophils, it enhances NADPH oxidase assembly and activation at the plasma membrane and simultaneously there is a pre-assembly of the neutrophil granules to the plasma membrane, which serves to pre-activate neutrophils (285). A regulated priming response induced by host- or bacterial- derived factors presents an effective immune surveillance mechanism that promotes host defense; however, an excessive priming response results in an excessive production of ROS, leading to host tissue damage (190).

It has been documented that periodontal pathogens can induce neutrophil phagocytosis and intracellular and extracellular ROS production. ROS produced by neutrophils in response to bacterial plaque formation in the oral cavity plays a crucial role in antimicrobial killing (286289). However excessive uncontrolled ROS leads to a variety of detrimental host effects, including: damage to DNA and proteins, interference with cell growth and cell cycle progression and induction of apoptosis of the gingival fibroblasts (286, 290-293). Additionally, ROS can impact signaling involved with osteoclastogenesis, which leads to bone destruction and periodontal disease development $(286,294)$. ROS can directly stimulate the degradation of extracellular matrix (ECM) through breakdown of glycosaminoglycan and matrix proteinases (286, 295-297). Clinical studies have shown that markers of oxidative stress found in saliva could be used as biomarkers for periodontal disease $(286,298-301)$. 
Periodontal pathogens like $P$. gingivalis, $T$. denticola, $F$. nucleatum and $A$.

actinomycetemcomitans challenge neutrophils and activate them to produce potent antimicrobial, but tissue-destructive compounds like ROS, MMPs, elastases and pro-inflammatory cytokines $(179,302-305)$. Studies performed by Jayaprakash et al determined that $P$. gingivalis (ATCC3277 strain) was effectively able to induce intracellular ROS production by human neutrophils, which led to only $\sim 35 \%$ survival post neutrophil challenge (306). Peptoanaerobacter stomatis ( $P$. stomatis), a newly appreciated oral pathogen, does not on its own induce much extracellular superoxide response by human neutrophils, however it has the ability to prime human neutrophils for an enhanced respiratory burst response to other stimuli, which may help the oral community (307). Although $P$ stomatis induced a robust intracellular respiratory burst, it did not contribute to the neutrophil's ability to effectively kill this organism (307). However, P. stomatis was susceptible to extracellular killing due to the release of antimicrobial contents from the neutrophil granules (307).

As an evasion strategy from oxygen-dependent antimicrobial responses by neutrophils, numerous pathogens have developed interference mechanisms to avoid phagocytosis, prevent the fusion of neutrophil granules to the phagosome, and target the NADPH oxidase complex assembly and activation. This targeting of the NADPH oxidase of both neutrophils and macrophages is observed by gram-negative, gram-positive, anaerobic and aerobic bacteria (127, $128,132,139,140,142,285,308-324)$.

In this chapter, we demonstrate how $F$. alocis manipulates the human neutrophils in order to evade oxygen-dependent killing mechanisms. These results suggest that $F$. alocis is capable of surviving in the neutrophil phagosome and mounts a minimal intracellular respiratory burst response. Additionally, the bacterium does not produce a significant superoxide release; however, F. alocis challenge can prime human neutrophils to an enhanced respiratory burst response. 


\section{$\underline{\text { Results }}$}

$\underline{F}$. alocis survives both intracellularly and extracellularly up to $4 \mathrm{~h}$ post-neutrophil challenge.

As neutrophils are function to recognize and kill their target, it is important to determine if neutrophils are effective in their ability to kill F. alocis or if the bacteria persist and survive. In order to determine $F$. alocis viability when challenged with neutrophils, we utilized BacLight viability dyes to assess the intracellular and extracellular bacterial viability. We challenged neutrophils with opsonized F. alocis for $0.5-2-4 \mathrm{~h}$ and then stained the bacteria using Syto9 (membrane-permeable fluorescent dye that stains all bacteria) and PI (membrane-impermeable fluorescent dye that detects damaged or dead bacteria), which can distinguish the viability of individual bacteria (Fig. 3-1 A). Our data revealed that after 30 min of $F$. alocis challenge, $\sim 65 \%$ remained viable inside the neutrophil, which increased to $\sim 82 \%$ at $2 \mathrm{~h}$, and then showed a nonsignificant decrease to $\sim 67 \%$ at $4 \mathrm{~h}$ (Fig. 3-1 B). However, when detecting extracellular viability of $F$. alocis, we saw a similar trend as with internal viable bacteria at $30 \mathrm{~min}(\sim 66 \%)$ and $2 \mathrm{~h}$ ( $\sim 71 \%)$, however a more pronounced decrease, although not statistically significant, in viability was observed at $4 \mathrm{~h}(\sim 41 \%)$ (Fig. 3-1 C). F. alocis is able to survive after 4 hours post neutrophil challenge, which indicates neutrophils may be ineffective at clearing this pathogen through both intracellular and extracellular killing mechanisms. However, neutrophils are effective at eliminating pathogens like Shigella, Listeria, and Salmonella through the degradation of bacterial virulence factors, their delivery of potent antimicrobials (e.g. neutrophil elastase) or oxidants to the bacterial-containing phagosome (143).

F. alocis induces minimal intracellular ROS but primes neutrophils.

Having established that $F$. alocis can survive intracellularly, our laboratory set out to determine the intracellular respiratory response employed by human neutrophils in response to $F$. alocis challenge. In order to assess this, our laboratory performed flow cytometry to determine the respiratory burst produced by neutrophils challenged with $F$. alocis, as detected by the oxidation of DCF by the presence of reactive oxygen species. In comparison with known 
intracellular ROS (iROS) stimulator $S$. aureus, both non-opsonized and opsonized viable $F$. alocis induced a minimal level of iROS, significantly less than opsonized S. aureus. However, opsonized heat-killed $F$. alocis induced a robust iROS response, similar to the level induced by opsonized $S$. aureus. Next, we sought to determine if upon $F$. alocis challenge, neutrophils generate ROS extracellularly.

Neutrophils are also capable of performing extracellular killing mechanisms through the targeting of neutrophil granule exocytosis and the assembly of the NADPH oxidase complex at the plasma membrane, resulting in the extracellular release of ROS. In this mechanism, they release ROS from their cell membrane and kill targets that are not internalized into a phagosomal compartment within the neutrophil, but instead reside in the extracellular space. In order to determine if $F$. alocis challenge of neutrophils stimulated extracellular production of superoxide, the amount of superoxide release was measured using a spectrophotometer to detect the oxidation of ferricytochrome C. Neutrophils challenged with $F$. alocis showed minimal superoxide production across a time course (5-15-30-60-90-120-150 mins) compared with fMLF, a moderate superoxide stimulator (Fig. 3-2). However, in our time course studies, we observed that F. alocis pre-treatment primes neutrophils to a secondary stimulus, as we saw a time-dependent increase in fMLF-stimulated superoxide production compared to fMLF alone, a response similar to that observed with a positive control for neutrophil priming, TNF- $\alpha$ plus fMLF (Fig. 3-2). This data also allowed us to conclude that $60 \mathrm{mins}$ is the optimal time point for neutrophil priming induced by $F$. alocis and that at later time points this response plateaus (Fig. 3-2).

Knowing that bacteria viability is an important determinant for $F$. alocis manipulation of intracellular ROS production, we next sought to determine if there was a difference in the production of extracellular superoxide. Similarly, to the studies performed with viable $F$. alocis (Fig. 3-2), we challenged neutrophils with heat-killed F. alocis at 60 mins time point (peak time observed in our time course in Fig. 3-2) and measured superoxide production. We observe that 
similar to live bacterium, there was no induction of superoxide production with heat-killed $F$. alocis alone. Furthermore, a similar priming effect was observed, as the results obtained with viable $F$. alocis (Fig. 3-2, 3-3 A). In order to determine if secreted bacterial factors present in the spent supernatants of $F$. alocis culture were sufficient to stimulate superoxide production, we also challenged neutrophils with the spent supernatants of $F$. alocis and compared to the response elicited by viable $F$. alocis. Our data showed that similarly to viable and heat-killed $F$. alocis, the spent supernatants of $F$. alocis induce minimal superoxide production, however they are effective at priming the neutrophil (Fig.3-3 B). These results help us to determine that $F$. alocis induces minimal extracellular ROS, however the bacteria are able to prime the fMLF-stimulated superoxide release.

F. alocis challenge of human neutrophils resulted in signaling through a TLR 2/6-dependent mechanism.

In order to combat organisms and particulates, neutrophils recognize and initiate binding of their target, undergo phagocytosis to engulf their target and then aim to control and kill using many of their potent antimicrobial killing mechanisms, eliminating threat to the host and maintaining homeostasis. In the context of our studies, we sought to characterize the human neutrophil ability to recognize, signal and employ its oxidative burst response to $F$. alocis challenge.

Neutrophils recognize $F$. alocis through TLR2, which triggers degranulation and cell migration (325). However, TLR2 can form heterodimer with either TLR1 or TLR6, depending on the stimuli. Additionally, due to the data we obtained showing that $F$. alocis challenge primes neutrophils, we chose a priming assay to further help us to characterize the signaling of TLR2. In order to determine whether $F$. alocis stimulates signaling through TLR $2 / 1$ or TLR $2 / 6$, we used commercially available agonists, $\mathrm{PAM}_{3} \mathrm{CSK}_{4}$ (TLR 2/1) and FSL-1 (TLR 2/6) as positive controls. $\mathrm{PAM}_{3} \mathrm{CSK}_{4}$ is a synthetic triacylated lipopeptide that can mimic bacterial lipopeptides through its acylated amino terminus, is known to be a potent activator of pro-inflammatory 
transcription factor, NF- $\kappa \mathrm{B}$, and is recognized by the TLR2/1 heterodimer. FSL-1 is a synthetic lipopeptide (derived from Mycoplasma salivarium) containing a diaglycerol structure, with diacylated cysteine resides and is recognized by the TLR2/6 heterodimer. Previous literature from Whitmore et al. observed that the $\mathrm{PAM}_{3} \mathrm{CSK}_{4}$ (TLR 2/1 agonist) was able to only prime neutrophils from certain donor populations to induce an enhanced respiratory burst response (284). However, neutrophils from all the donors exposed to FSL-1 (TLR 2/6 agonist) showed a primed neutrophil phenotype with an enhanced respiratory burst response (284) Using genotypic analysis, it was revealed that certain human patients have a single nucleotide polymorphism in TLR1 (rs5743618) that allows for an enhanced priming response by neutrophils, as their neutrophils have enhanced TLR1 surface expression (284). Therefore, we tested our donor population to identify high and low primers depending on the responses we obtained when neutrophils were exposed to $\mathrm{PAM}_{3} \mathrm{CSK}_{4}$. Similarly to results obtained by Whitmore et al., we saw that $\mathrm{PAM}_{3} \mathrm{CSK}_{4}$ induced priming in only certain neutrophil donor populations, however FSL1 induced priming in neutrophils from all of our donor pool (Fig. 3-4 A-B).

After exposing neutrophils to $\mathrm{PAM}_{3} \mathrm{CSK}_{4}$ or FSL-1 or challenging them with $F$. alocis for 60 min (based on the peak response we observed with our priming studies in Fig. 3-2), we determined the superoxide production. Our data showed that neutrophils primed with F.alocis and then stimulated with fMLF showed a robust superoxide response to the same extent as the priming positive control, TNF- $\alpha$ + fMLF and the TLR 2/6 agonist FSL-1 + fMLF (Fig.3-4 A-B). However, when neutrophils were primed with the $\mathrm{PAM}_{3} \mathrm{CSK}_{4}$ prior to exposure to fMLF, we observed a variable induction of superoxide response, as some donors showed a high priming response and some showed a low priming response to the TLR 2/1 agonist, $\mathrm{PAM}_{3} \mathrm{CSK}_{4}$ (Fig. 3-4 A-B). This data led us to conclude that $F$. alocis may signal through at TLR 2/6-dependent mechanism, based on the ability to prime neutrophils to the same extent as TLR 2/6 agonist, FSL1. 
Similar percent recruitment of membrane and some of the cytosolic NADPH oxidase components to $F$. alocis and HK-F. alocis phagosomes.

Following effective phagocytosis, neutrophils rely on the rapid assembly and activation of the NADPH oxidase complex, to effectively utilize its oxygen-dependent killing mechanisms. The NADPH cytosolic components (p47 $7^{\text {phox }}, \mathrm{p} 67^{\text {phox }}, \mathrm{p} 40^{\text {phox }}$, GTP-Rac2) upon cell stimulation will be translocated and phosphorylated to dock on the cell phagosome or plasma membrane and bind along with membrane components (cytochrome b558 consisting of gp91 phox, p22 ${ }^{\text {phox }}$ ). The translocation and phosphorylation of these components is a rapid process, occurring within 30 seconds of cell stimulation. Once the complex is fully assembled and phosphorylated, only then will the oxidative burst response will be initiated. After initiating the oxygen-dependent killing responses, the cytosolic components will be de-phosphorylated and disassemble from the phagosomal membrane, ensuring the oxidative burst response is halted as it should be tightly controlled to ensure unnecessary host damage.

Therefore, since viable, but not heat-killed, F. alocis induced minimal intracellular ROS production, we sought to determine if this was due to the ability of $F$. alocis to prevent or delay recruitment of all or some of the NADPH oxidase components. In order to more closely determine the role of NADPH oxidase assembly upon neutrophil challenge with $F$. alocis, we used confocal immunostaining to detect oxidase components and their localization to the bacterial-containing phagosome. Several studies in human and mice neutrophils show that different pathogenic microbes can prevent or delay ROS production when $40 \%$ or less of the oxidase components were recruited to the phagosomal structure $(127,128,132,139,140,142$, $285,308-324)$.

We first assessed the recruitment of the membrane components gp91 ${ }^{\text {phox }}$ (Fig. 3-5A) and p22 $2^{\text {phox }}$ (Fig. 3-6 A) at an early time point using neutrophils challenged for 15 mins with CFSElabeled opsonized viable or CFSE-labeled opsonized heat-killed F. alocis. Our data showed that gp9 $91^{\text {phox }}$ was effectively recruited to the live $(67 \%)$ or heat-killed $(70 \%)$ bacteria-containing 
phagosomes. Also, p22 $2^{\text {phox }}(65 \%, 69 \%)$ was effectively recruited to live $(65 \%)$ or heat-killed (69\%) bacteria-containing phagosomes (Fig. 3-5-6). There was a significant enhancement in gp9 $91^{\text {phox }}$ and $\mathrm{p} 22^{\text {phox }}$ positive phagosomes exposed to the positive control used for this assay, opsonized Zymosan (95\% gp91 ${ }^{\text {phox }}, 90 \%$ p22 $\left.2^{\text {phox }}\right)$, which is known to effectively stimulate efficient oxidase assembly, at early time points $(127,309,317,324)$. Recruitment of $\mathrm{p} 47^{\text {phox }}$ is essential for a functional oxidase, and several pathogenic bacteria prevent its recruitment to compromise the ROS production $(127,309,311,321)(314,316)(308,314)$ As the assembly, activation and disassembly of the NADPH oxidase complex is a dynamic process, we sought to determine the recruitment of the components at 15-30-60 min time points. At later time points, it is expected that the NADPH oxidase complex will deactivate and disassemble. Hence, we assessed recruitment of $\mathrm{p} 47^{\text {phox }}$ to live or heat-killed $F$. alocis phagosomes at different time points of 15-30 and 60 min post challenge (Fig. 3-7A). At the 15-30 mins time point, it was observed that $\mathrm{p} 47^{\text {phox }}$ was effectively recruited to the phagosomal membrane containing viable $(60 \%-15$ mins, 65\%-30 mins) or heat-killed (60\%-15 mins, 65\%-30 mins) (Fig. 3-7 B). F. alocis, to a similar extent. Positive control Zymosan more effectively recruited $\mathrm{p} 47^{\text {phox }}$ to its phagosome compared with viable or heat-killed $F$. alocis at 15 mins (96\%) (Fig. 3-7 B). However, at 30-60 mins, as expected based on previous literature (127), $\mathrm{p} 47^{\text {phox }}$ begins to dissociate from the phagosomal membrane containing Zymosan (31\%-15 mins, 18\%-60 mins) (Fig. 3-7 B). Our data showed that at 60 mins, $\mathrm{p} 47^{\text {phox }}$ starts to dissociate from the phagosome in neutrophils challenged with viable $F$. alocis (42\%), however this trend is not the same as with the heat-killed $F$. alocis (52\%), which retains $\mathrm{p} 47^{\text {phox }}$ to the phagosome (Fig. 3-7 B).

It was observed that $\mathrm{p} 67^{\text {phox }}$ was observed to be effectively recruited to the phagosomal membrane containing viable $(63 \%)$ or heat-killed (64\%) F. alocis, to a similar extent (Fig. 3-8). Positive control Zymosan more effectively recruited $\mathrm{p} 67^{\text {phox }}$ to its phagosome compared with viable or heat-killed F. alocis at 15 mins (94\%) (Fig. 3-8). 
However, when we assessed the recruitment of $\mathrm{p} 40^{\text {phox }}$ to the $F$. alocis-containing phagosome at 15 mins, we saw significant differences in the percent positive phagosomes between viable and heat-killed $F$. alocis, $46 \%$ and 60\%, respectively (Fig. 3-9 A, B). Positive control Zymosan more effectively recruited $\mathrm{p} 40^{\text {phox }}$ to its phagosome compared with viable or heat-killed F. alocis at 15 mins (83\%) (Fig. 3-9 B). This delay in recruitment of $\mathrm{p} 40^{\text {phox }}$ to the viable $F$. alocis-containing phagosome could help explain the impaired induction of the intracellular respiratory burst response, as compared with the heat-killed $F$. alocis-containing phagosome. At the 30-60 mins time point, we saw effective recruitment of $\mathrm{p} 40^{\text {phox }}$ to both the viable (65\%-30 mins, 66\%-30 mins) and heat-killed (65\%-30 mins, 75\%-60 mins) $F$. alocis phagosomes (Fig. 3-9 B). Similar to what was observed with the disassembly of p47 phox , our data showed that $\mathrm{p} 40^{\text {phox }}$ begins to dissociate from the Zymosan containing phagosomes (127) at 30 mins (31\%) and 60 mins (13\%), as expected (Fig. 3-9 B). While the p47 phox is disassembling from the bacterial-containing phagosome at later time points, as expected, the $\mathrm{p} 40^{\mathrm{phox}}$ is retained on the bacterial-containing phagosome.

Impaired Rac activation when neutrophils were challenged with $F$. alocis.

Another important cytosolic component of the NADPH oxidase complex that is activated and recruited to the bacterial-containing phagosome is the small GTP-bound Rac2. As it was determined that GTPase Rac is critical for regulating and activating the NADPH oxidase complex, we next wanted to determine its recruitment capabilities to an F. alocis-containing neutrophil phagosome. We performed a time course (6-15-30-60 mins) study using a G-protein activation assay to detect the GTP-bound form of Rac from neutrophil lysates collected from neutrophils challenged with opsonized viable $F$. alocis and opsonized heat-killed $F$. alocis. As fMLF is a known Rac activator after exposure at early time points, we used this as our positive control for induction. Our data suggests that there is an impaired Rac activation with viable $F$. alocis compared to heat-killed F. alocis at the 30 min time point (Fig. 3-10). The ineffective Rac 
activation could help to explain the impaired induction of the intracellular respiratory burst response seen with the viable $F$. alocis.

\section{Discussion}

Neutrophil oxidative killing mechanisms are employed when these cells become activated by a stimulus at a site of injury or inflammation. It has been implicated that bacterial survival is dependent on manipulating neutrophils ability to perform phagocytosis and induce an ROS response (127). As neutrophils effectively employ their respiratory burst response upon challenge with numerous bacterial and fungal pathogens, these pathogens must utilize their stress response mechanisms to ensure avoidance or evasion of the neutrophil, leading to their persistence and survival (326). It is known that neutrophils are efficiently recruited and found in the oral cavity, therefore the studies into the killing mechanisms at this site are important to determine in the context of $F$. alocis infection and further periodontal disease. We determined that $F$. alocis manipulates neutrophil oxygen-dependent killing mechanisms to promote its survival both intracellularly and extracellularly.

Bacteria are known to employ a variety of mechanisms to counter-act killing mechanisms employed by neutrophils, including: inhibiting the oxidative burst in the phagosome ( $F$. tularensis, H. pylori), inhibiting the V-ATPase activity, blocking neutrophil granule fusion with the phagosomal compartment (Mycobacteria, S. pyogenes, Salmonella), using catalase to disrupt the production of oxidative metabolites, employing proteases to target host factors and also producing pore-forming enzymes to allow for phagosomal escape (F. tularensis, S. pyogenes). Some bacterial pathogens are able to effectively replicate inside neutrophils, these include: $A$. phagocytophilium, C. trachomatis, L. pneumophila $(143,144)$.

After internalization by neutrophils, Staphylococcus aureus upregulates agr virulence genes which allows for it to survive inside the neutrophil, potentially through the use of membrane lytic toxins $(326,327)$. Catalase aids $S$. aureus survival by protecting the bacterium 
once it has been internalized, through the conversion of hydrogen peroxide to oxygen and water $(328,329)$. S. aureus secretes cytolytic toxins to protect itself from killing both inside the phagosome and in the extracellular space (329). Through the use of proteins that can sense oxidation and in turn act as transcriptional regulator of antioxidant enzyme production, S. aureus can facilitate defense mechanisms against neutrophils (328). Periodontal pathogen, $A$. actinomycetemcomitans, damages neutrophils through the production of leukotoxins, allowing them to dampen the immune responses and cause more destruction of periodontal tissue (330). Additionally, through sensing the production of hydrogen peroxide, A. actinomycetemcomitans upregulates genes that allow this bacterium to effectively resist neutrophil killing (331). Bacteria can impair numerous steps involved in the activation and use of oxidative-killing mechanisms by neutrophils, highlighting the importance of elucidating these secreted virulence factors and their functions (332). As little is known regarding the virulence factors $F$. alocis possesses, it is possible that the survival of our bacterium is mediated through virulence genes, proteins or enzymes.

Through the manipulation of granule targeting and fusion to the bacterial phagosome, $N$. gonorrhoeae avoids killing by neutrophils, remaining viable intracellularly $(319,333,334)$. Studies performed in our laboratory reveal that specific and azurophil granule fusion to the membrane of the $F$. alocis-containing phagosome is impaired, which can reduce the antimicrobial capacities of neutrophils targeting the phagosome, further promoting bacterial survival.

Additionally, pathogens can use superoxide dismutase (SOD) or catalase to mediate the effects of ROS production, as they are capable of catabolysis and detoxification $(326,335)$. As observed with $S$. aureus, it is critical for bacterial survival to demonstrate an effective means for reduce or inhibit ROS production. S. aureus scavenges and neutralizes ROS (superoxide and $\mathrm{H}_{2} \mathrm{O}_{2}$ ) through the use of SODs (SodA and Sod M) and catalases (336-338). Additionally, $S$. aureus uses staphyloxanthin to evade neutrophil killing as it serves as an antioxidant against peroxide and singlet oxygen $(329,339)$. Psuedomonas aeruginosa is able to effectively scavenge 
neutrophil ROS (hydrogen peroxide and superoxide) through the kynurenine pathway, which catabolizes tryptophan, in order to evade the innate immune response (340). It is possible that $F$. alocis may utilize superoxide reductase(64) to scavenge and neutralize ROS, as an effective survival strategy.

Another mechanism of avoidance of neutrophil killing mechanisms is observed with microbial siderophores, which function in scavenging iron, neutralizing the neutrophils from effectively utilizing their antimicrobial radical oxygen species production (341). Neutrophils also rely on iron for the production of their oxidative burst response and their pro-inflammatory mediators (like MPO, NADPH oxidase) are part of the heme family proteins $(341,342)$. As iron is a critical nutrient for certain microbes, they employ an iron chelator to steal iron from their host once the host induces a stressful hypoferremic state of low iron $(341,343)$. E. coli encodes for Ent, a siderophore, that is observed to inhibit neutrophil functions, like ROS (341). Bacterial siderophores have far-reaching implications, as they are not merely responsible for the scavenging of iron, but also can be involved in the production of immunoregulatory metabolites (341). Through the use of small molecule thiols, which are highly reactive with the ROS produced by neutrophils, bacteria generate thiols as a defense strategy to combat neutrophil killing capabilities by $\operatorname{ROS}(328,344-346)$. As we see that $F$. alocis has iron binding domains (64), this could aid the bacterium in scavenging iron, which may effectively promote its survival despite neutrophils employing their killing mechanisms.

Due to the rapid assembly of the NADPH oxidase complex, most microbes effectively internalized by neutrophils will be killed within 30-60 min $(143,347)$. Additionally, it has been reported that the NADPH oxidase assembly and activation play a role in inflammatory disease development (like rheumatoid arthritis) $(135,348-350)$. As an evasion and survival strategy from the effects of the potent ROS response by neutrophils, numerous pathogens have developed interference mechanisms targeted at the NADPH oxidase complex assembly and activation (See Table 1). F. alocis appears to behave similarly to the microorganism S. aureus and the yeast 
particle Zymosan, in its ability to effectively induce recruitment of NADPH oxidase components to the phagosome. Additionally, as expected based on the NADPH components being effectively recruited, S. aureus produces a robust intracellular respiratory burst response. However, in our studies, F. alocis manipulates neutrophils, as the recruitment of the complex to the phagosome is unaffected, yet a minimal respiratory burst response is produced.

Previous studies highlight the importance of NADPH oxidase assembly for activation and induction of the respiratory burst response by neutrophils (132). In our studies, there does not seem to be an impaired recruitment of the NADPH oxidase components to the bacterialcontaining phagosome, however challenging neutrophils with $F$. alocis results in a minimal respiratory burst response. Therefore, it may be important to consider if the $F$. alocis is able to manipulate the neutrophils to retain components, that may be dysfunctional, to the phagosomal compartment, as observed in our studies with $\mathrm{p} 40^{\text {phox }}$. Additionally, there could be an impairment in the recycling in of "fresh" subunits, which is required for a sustained oxidase activation. Through the kinetic studies performed by Karimi et al, it was determined that the proteins must all be activated simultaneously, as even a short delay of 10 seconds resulted in decreased activity (351). In our studies, F. alocis could be manipulating the timing of the activation of the components of the NADPH oxidase complex, leading to an ineffective respiratory burst response. Neither virulent nor avirulent strains of $F$. tularensis were effectively killed by neutrophils and $\sim 70 \%$ of internalized bacteria remained viable $2-4-8 \mathrm{hrs}$ after neutrophil challenge (128). The live vaccine strain of $F$. tularensis (LVS) induces a minimal respiratory burst response compared to formalin or periodate- killed LVS as assessed by NBT staining (347). LVS may impact a signaling pathway involved in ROS production, as it showed that it can block NADPH oxidase activity once it is triggered by a known stimulus like PMA or zymosan. Given the results observed by LVS, it is possible that in our studies, $F$. alocis can trigger the translocation of the NADPH oxidase complex, however it can block the enzyme activity. 
The NADPH oxidase complex is a finely-tuned machine and can operate through varied mechanisms of assembly-activation-disassembly-inactivation, depending on the stimuli encountered. Numerous studies point to factors involved to impact component recruitment and phosphorylation, all of which can affect the magnitude of the respiratory burst produced, which can help to explain our observations with $F$. alocis. Interestingly, it has been reported in studies with receptor-mediated stimulation that the phosphorylation of both $\mathrm{p} 47^{\text {phox }}$ and $\mathrm{p} 67^{\text {phox }}$ could be dissociated from the function of translocation or that a certain degree of phosphorylation must be attained before translocation can occur (352). It has also been determined that translocation of phox proteins requires a phosphorylated $\mathrm{p} 47^{\text {phox }}$ at multiple serine residue sites $(143,317)$; which could lead us to interpret that despite effective translocation of our NADPH oxidase components, phosphorylation status has yet to be determined, which could help to explain the low iROS response produced by neutrophils challenged with $F$. alocis. Also, studies have suggested that phosphorylation may not be the only protein modification on the NADPH oxidase components that is necessary for translocation and activation of the NADPH oxidase complex, as calcium release and electron transfer may also play a role (352-354). Additionally, it was reported that the NADPH oxidase complex can assemble properly, however an impairment in the electron transfer resulted in minimal production of $\operatorname{ROS}(276,355)$.

Our results also suggest that viability could be a factor in the assembly, activation, and disassembly of the $\mathrm{p} 40^{\mathrm{phox}}$ component, as heat-killed $F$. alocis retained this component on the phagosome to induce a more robust and sustained downstream ROS response. Additionally, viable $F$. alocis may be required for disassembly and inactivation of the oxidase complex, as by 60 mins the heat-killed $F$. alocis retained $\mathrm{p} 47^{\text {phox }}$ to the phagosomes, where viable $F$. alocis did not. Studies performed by Keith et al with B. cenocepacia demonstrated that viable bacteria could delay assembly and recruitment of the NADPH oxidase complex, however heat-inactivated bacteria could not (320). p40 phox ability to effectively bind to PI3Ps is crucial for necessary highlevel intracellular production of ROS effective in killing (142), which may be the case in the 
context of $F$. alocis challenge, as there is minimal ROS production and impaired $\mathrm{p} 40^{\text {phox }}$

recruitment at 15 mins. At the later time points, $\mathrm{p} 40^{\text {phox }}$ is effectively recruited and retained, but it is unclear whether it is bound to the PI3P. The importance of this binding interaction was demonstrated in mice lacking $\mathrm{p} 40^{\text {phox }}$ or a mutant PX domain, where it was observed that in vitro oxidant-dependent killing was reduced to the level of complete absence of NADPH activity (142).

Kinase signaling, like PKC and PI3P, is involved in phosphorylation of the NADPH oxidase components (356), therefore the possibility exists that $F$. alocis could manipulate these pathways. The GTP-Rac is deemed necessary for the activation of the kinases that ensure phosphorylation of oxidase components (356). Multiple pathways have been implicated to be involved in regulating Rac2 and how its deficiency leads to deficient superoxide production (136, 357-359). Downstream effector neutrophil functions are regulated by Rac2, especially those involved in chemotaxis and Fc receptor signaling (136). Using Rac-2 deficient studies, it was determined that GTPase Rac is critical for regulating the NADPH oxidase and that the GTPbound form Rac is necessary for activation and further catalytic functions of the complex (145, 356). As our studies revealed an impaired activation of GTP-bound Rac induced by neutrophils challenged with viable $F$. alocis, this could help to explain a way that our bacterium manipulates neutrophils to produce a minimal respiratory burst response.

The final battle of host and pathogen results in either success of the host, by elimination of the pathogen or success of the pathogen, by survival and persistence in the host. We saw that F. alocis effectively over time remains viable and resistant to intracellular and extracellular oxygen-dependent killing mechanisms utilized by neutrophils. This indicates further implications for periodontal disease development, as neutrophils may lose the host-pathogen battle, leaving room for the manipulative $F$. alocis to survive and persist while promoting chronic inflammation- 
Table 1. NADPH oxidase component assembly and activation impairment upon challenge

\begin{tabular}{|c|c|c|c|c|c|c|c|c|}
\hline $\begin{array}{l}\text { Microorganis } \\
\text { m or Stimulus }\end{array}$ & $\begin{array}{l}\text { Gram } \\
\text { stain } \\
\text { identifi } \\
\text { cation }\end{array}$ & $\begin{array}{l}\text { Mode } \\
\text { of } \\
\text { Respir } \\
\text { ation }\end{array}$ & $\begin{array}{l}\text { Source of } \\
\text { opsonin }\end{array}$ & $\begin{array}{l}\text { Host } \\
\text { species }\end{array}$ & $\begin{array}{c}\text { \% Positive } \\
\text { Recruitment } \\
\text { (as assessed by } \\
\text { Immunoblotting } \\
\text { (IB) or } \\
\text { Immunofluorescen } \\
\text { ce }(\text { IF }) \text { ) } \\
\end{array}$ & $\begin{array}{l}\text { Phosphorylat } \\
\text { ion (as } \\
\text { assessed by } \\
\text { Immunoblott } \\
\text { ing) }\end{array}$ & $\begin{array}{c}\text { Proposed } \\
\text { factor(s)/mechanism(s) for } \\
\text { impairment }\end{array}$ & $\begin{array}{l}\text { Referenc } \\
\quad \text { es }\end{array}$ \\
\hline $\begin{array}{c}\text { Anaplasma } \\
\text { phagocytophil } \\
\text { um }\end{array}$ & - & $\begin{array}{c}\text { Aerobi } \\
\quad \mathrm{c}\end{array}$ & $\begin{array}{l}\text { Human } \\
\text { serum }\end{array}$ & $\begin{array}{c}\text { Human } \\
\text { neutrophil } \\
\text { s }\end{array}$ & $\begin{array}{c}\text { IF: gp91phox } \\
(\sim 15 \%), \text { p22phox } \\
(\sim 5 \%) \text { at } 30,60,90 \\
\text { mins }\end{array}$ & N/A & $\begin{array}{l}\text { Modification of promoter } \\
\text { activity for gp91phox; } \\
\text { bacterium resides in } \\
\text { protective vacuole once } \\
\text { internalized }\end{array}$ & $\begin{array}{c}(132, \\
140,315)\end{array}$ \\
\hline $\begin{array}{l}\text { Burkholderia } \\
\text { cenocepacia }\end{array}$ & - & $\begin{array}{c}\text { Aerobi } \\
\mathrm{c}\end{array}$ & $\begin{array}{c}\text { Non- } \\
\text { opsonized }\end{array}$ & $\begin{array}{l}\text { Macropha } \\
\text { ge RAW } \\
264.7 \text { and } \\
\text { ANA-1 } \\
\text { cell line }\end{array}$ & $\begin{array}{l}\text { IF: p22phox and } \\
\text { p40phox }(20 \%) \text { up } \\
\text { to } 4 \text { hrs post- } \\
\text { infection } \\
\text { IF: gp91phox and } \\
\text { p47phox in } B \text {. } \\
\text { cenocepacia } \\
\text { ( } \Delta \text { atsR })(\sim 5 \%) \\
\text { compared to } \\
(\sim 60 \%) \text { in } B . \\
\text { cenocepacia } \\
(\Delta \text { atsR } \Delta \text { T } 6 \mathrm{SS})\end{array}$ & $\begin{array}{c}\text { Defective } \\
\text { phosphorylati } \\
\text { on in } \Delta \text { atsR } \\
(\sim 3 \\
\text { normalized } \\
\text { relative units }) \\
\text { compared to } \\
(\sim 20 \\
\text { normalized } \\
\text { relative units }) \\
\text { in } \\
\Delta \text { atsR } \Delta \text { T6SS }\end{array}$ & $\begin{array}{c}\text { Type } 6 \text { Secretion System } \\
\text { (T6SS) }\end{array}$ & $\begin{array}{l}(320, \\
322)\end{array}$ \\
\hline
\end{tabular}




\begin{tabular}{|c|c|c|c|c|c|c|c|c|}
\hline $\begin{array}{c}\text { Coxiella } \\
\text { burnetii } \\
\text { (NMII) }\end{array}$ & - & $\begin{array}{l}\text { Aerobi } \\
\quad \mathrm{c}\end{array}$ & $\begin{array}{l}\text { Human } \\
\text { serum }\end{array}$ & $\begin{array}{c}\text { Human } \\
\text { neutrophil } \\
\mathrm{s}\end{array}$ & $\begin{array}{l}\text { IB: Prevents } \\
\text { recruitment of } \\
\text { p47phox and } \\
\text { p67phox compared } \\
\text { to } S . \text { aureus or } \\
\text { PMA at } 30 \text { mins }\end{array}$ & $\mathrm{N} / \mathrm{A}$ & $\begin{array}{c}\text { Inhibition of cytosolic } \\
\text { oxidase components on the } \\
\text { phagosome (Mechanism } \\
\text { unknown) }\end{array}$ & (311) \\
\hline $\begin{array}{l}\text { Escherichia } \\
\text { coli (F18) }\end{array}$ & - & $\begin{array}{l}\text { Faculta } \\
\text { tively } \\
\text { Anaero } \\
\text { bic }\end{array}$ & $\begin{array}{c}\text { Non- } \\
\text { opsonized }\end{array}$ & $\begin{array}{c}\text { Mouse } \\
\text { peritoneal } \\
\text { macropha } \\
\text { ges from } \\
\text { WT } \\
\text { C57BL/6 } \\
\text { or MyD88 } \\
\text {-/- mice }\end{array}$ & $\begin{array}{c}\text { IF: Prevents } \\
\text { recruitment of } \\
\text { p47phox and } \\
\text { p22phox in MyD88 } \\
\text {-/- mice } \\
\text { macrophages } \\
\text { compared to WT } \\
\text { mice macrophages }\end{array}$ & $\begin{array}{c}\text { Defective } \\
\text { phosphorylati } \\
\text { on of } \\
\text { p47phox in in } \\
\text { MyD88 -/- } \\
\text { compared to } \\
\text { WT at 5,15, } \\
30 \text { mins }\end{array}$ & MyD88 signaling & (321) \\
\hline $\begin{array}{c}\text { Escherichia } \\
\text { coli } \\
\text { (containing } \\
\text { pMAL-C2X } \\
\text { vector } \\
\text { encoding } \\
\text { maltose } \\
\text { binding } \\
\text { protein) }\end{array}$ & - & $\begin{array}{l}\text { Faculta } \\
\text { tively } \\
\text { Anaero } \\
\text { bic }\end{array}$ & $\begin{array}{c}\text { Non- } \\
\text { opsonized }\end{array}$ & $\begin{array}{c}\text { Human } \\
\text { neutrophil } \\
\mathrm{s}\end{array}$ & $\begin{array}{l}\text { IF: gp91phox and } \\
\text { p22phox }(\sim 50- \\
60 \%) \text { at } 30,60,90 \\
\text { mins }\end{array}$ & N/A & N/A & (140) \\
\hline $\begin{array}{l}\text { Escherichia } \\
\text { coli }(D H 5-\alpha)\end{array}$ & - & $\begin{array}{l}\text { Faculta } \\
\text { tively } \\
\text { Anaero } \\
\text { bic }\end{array}$ & $\begin{array}{c}\text { Non- } \\
\text { opsonized }\end{array}$ & $\begin{array}{l}\text { Macropha } \\
\text { ge RAW } \\
264.7 \text { and } \\
\text { ANA-1 } \\
\text { cell line }\end{array}$ & $\begin{array}{l}\text { IF: p22phox and } \\
\text { p40phox }(100 \%)\end{array}$ & N/A & N/A & $\begin{array}{r}(320, \\
322)\end{array}$ \\
\hline
\end{tabular}




\begin{tabular}{|c|c|c|c|c|c|c|c|c|}
\hline $\begin{array}{l}\text { Francisella } \\
\text { novicida }\end{array}$ & - & $\begin{array}{c}\text { Aerobi } \\
\mathrm{c} \text { and } \\
\text { Faculta } \\
\text { tively } \\
\text { Anaero } \\
\text { bic }\end{array}$ & $\begin{array}{l}\text { Autologo } \\
\text { us serum }\end{array}$ & $\begin{array}{l}\text { Human } \\
\text { neutrophil } \\
\text { s and } \\
\text { Monocyte } \\
\text {-derived } \\
\text { macropha } \\
\text { ges }\end{array}$ & $\begin{array}{c}\text { IF: Neutrophils- } \\
\text { p47phox in } \\
\Delta \text { ABCH }(\sim 10 \%) \\
\text { compared to }(\sim 60- \\
85 \%) \text { in } \triangle \text { acpA and } \\
\text { WT strain at } 5,15, \\
30 \text { mins; } \\
\text { MDMs- p47phox in } \\
\Delta \text { acpA and WT } \\
\text { strain }(\sim 10 \%) \\
\text { compared to }(\sim 25, \\
45 \%) \text { in } \triangle \mathrm{ABCH} \\
\text { strain at } 30,60 \\
\text { mins }\end{array}$ & $\begin{array}{l}\text { Neutrophils: } \\
\text { Defective } \\
\text { phosphorylati } \\
\text { on of } \\
\text { p47phox and } \\
\text { p40phox in } \\
\text { WT } \\
\text { compared to } \\
\triangle \mathrm{ABCH} \\
\text { strain at } 15 \\
30 \text { mins }\end{array}$ & Acid phosphatase proteins & (309) \\
\hline $\begin{array}{l}\text { Francisella } \\
\text { tularensis }\end{array}$ & - & $\begin{array}{c}\text { Aerobi } \\
\mathrm{c}\end{array}$ & $\begin{array}{l}\text { Autologo } \\
\text { us serum }\end{array}$ & $\begin{array}{c}\text { Human } \\
\text { neutrophil } \\
\text { s }\end{array}$ & $\begin{array}{l}\text { IF: gp91phox and } \\
\text { p22phox in LVS } \\
(\sim 10-20 \%) \\
\text { IF: Prevents } \\
\text { recruitment of } \\
\text { gp91phox and } \\
\text { p22phox in Schu } \\
\text { S4 and } 1547-57 \text { at } \\
15 \text { mins } \\
\end{array}$ & $\mathrm{N} / \mathrm{A}$ & $\begin{array}{l}\text { Regulatory factor fevR, } \\
\text { sustained accumulation of } \\
\text { dysfunctional oxidase } \\
\text { complexes }\end{array}$ & $\begin{array}{r}(132, \\
139)\end{array}$ \\
\hline $\begin{array}{c}\text { Helicobacter } \\
\text { pylori }\end{array}$ & - & $\begin{array}{l}\text { Microa } \\
\text { erophil } \\
\text { ic }\end{array}$ & $\begin{array}{l}\text { Autologo } \\
\text { us serum }\end{array}$ & $\begin{array}{c}\text { Human } \\
\text { neutrophil } \\
\text { s }\end{array}$ & $\begin{array}{l}\text { IF: p47phox, } \\
\text { p67phox and } \\
\text { gp91phox ( 20- } \\
30 \%)\end{array}$ & $\mathrm{N} / \mathrm{A}$ & $\begin{array}{c}\text { Disruption of oxidase } \\
\text { component targeting } \\
\text { through accumulation of } \\
\text { superoxide anions in } \\
\text { extracellular space, not } \\
\text { phagosomes (Opsonins and } \\
\text { formalin-resistant and heat- } \\
\text { sensitive bacterial surface } \\
\text { factors) }\end{array}$ & $\begin{array}{l}(127, \\
309)\end{array}$ \\
\hline
\end{tabular}




\begin{tabular}{|c|c|c|c|c|c|c|c|c|}
\hline $\begin{array}{c}\text { Klebsiella } \\
\text { pneumoniae }\end{array}$ & - & $\begin{array}{l}\text { Faculta } \\
\text { tively } \\
\text { Anaero } \\
\text { bic }\end{array}$ & $\begin{array}{c}\text { Non- } \\
\text { opsonized }\end{array}$ & $\begin{array}{l}\text { Rat } \\
\text { alveolar } \\
\text { macropha } \\
\text { ges }\end{array}$ & $\begin{array}{l}\text { IB: Prevents } \\
\text { recruitment of } \\
\text { p47phox with } \\
\text { bacteria alone } \\
(100 \%) \text { compared } \\
\text { to } K \text {. pneumoniae } \\
\text { without PGE2 } \\
(25 \%) \text { at } 5 \text { mins }\end{array}$ & $\begin{array}{c}\text { Inhibits } \\
\text { p47phox } \\
\text { phosphorylati } \\
\text { on compared } \\
\text { to } K \text {. } \\
\text { pneumoniae } \\
\text { without } \\
\text { PGE2 at } 5 \\
\text { mins }\end{array}$ & $\begin{array}{c}\text { Prostaglandin receptor } \\
\text { signaling }\end{array}$ & $(323)$ \\
\hline $\begin{array}{l}\text { Leishmania } \\
\text { donovani }\end{array}$ & $\mathrm{N} / \mathrm{A}$ & $\begin{array}{l}\text { Faculta } \\
\text { tively } \\
\text { Anaero } \\
\text { bic }\end{array}$ & $\begin{array}{c}10 \% \\
\text { human } \\
\text { C8- } \\
\text { deficient } \\
\text { serum }\end{array}$ & $\begin{array}{c}\text { Mouse } \\
\text { macropha } \\
\text { ges }\end{array}$ & $\begin{array}{c}\text { IF: Prevents } \\
\text { recruitment in with } \\
\text { LPG-defective } \\
\text { strain }(\sim 40 \%) \\
\text { compared to WT } \\
(\sim 80 \%) \text { at } 15,30, \\
60 \text { mins }\end{array}$ & $\begin{array}{c}\text { Defective } \\
\text { phosphorylati } \\
\text { on of } \\
\text { p47phox at } \\
15,30 \text { mins }\end{array}$ & $\begin{array}{c}\text { Inhibition of } \\
\text { phagolysosome biogenesis } \\
\text { through surface glycolipid } \\
\text { lipophosphoglycan }\end{array}$ & $\begin{array}{c}(128, \\
309,310)\end{array}$ \\
\hline $\begin{array}{c}\text { Listeria } \\
\text { monocytogene } \\
\text { s }\end{array}$ & + & $\begin{array}{c}\text { Aerobi } \\
\mathrm{c}\end{array}$ & $\begin{array}{c}\text { Non- } \\
\text { opsonized }\end{array}$ & $\begin{array}{l}\text { Human } \\
\text { macropha } \\
\text { ges }\end{array}$ & $\begin{array}{c}\text { IF: p47phox, } \\
\text { p67phox and } \\
\text { p22phox }(\sim 30 \%) \\
\text { for cytolysin } \\
\text { mutant compared to } \\
\text { WT }(\sim 70 \%) \text { at } 30 \\
\text { mins }\end{array}$ & $\mathrm{N} / \mathrm{A}$ & $\begin{array}{c}\text { Production of } \\
\text { phospholipases C and the } \\
\text { pore-forming cytolysin } \\
\text { listeriolysin O protein } \\
\text { allows for escape from the } \\
\text { phagosome }\end{array}$ & $\begin{array}{l}(314, \\
316)\end{array}$ \\
\hline $\begin{array}{c}\text { Neisseria } \\
\text { gonorrhoeae }\end{array}$ & - & $\begin{array}{l}\text { Aerobi } \\
\text { c and } \\
\text { Faculta } \\
\text { tively } \\
\text { Anaero } \\
\text { bic }\end{array}$ & $\begin{array}{c}\text { Human } \\
\text { serum }\end{array}$ & $\begin{array}{c}\text { Human } \\
\text { neutrophil } \\
\text { s }\end{array}$ & $\begin{array}{l}\text { IF: p47phox and } \\
\text { p67phox }(\sim 40 \%)\end{array}$ & $\mathrm{N} / \mathrm{A}$ & $\begin{array}{l}\text { Opacity (Opa)-Associated } \\
\text { Protein Expression }\end{array}$ & (132) \\
\hline
\end{tabular}




\begin{tabular}{|c|c|c|c|c|c|c|c|c|}
\hline $\begin{array}{c}\text { Salmonella } \\
\text { typhimurium }\end{array}$ & - & $\begin{array}{c}\text { Aerobi } \\
\mathrm{c}\end{array}$ & $\begin{array}{c}\text { Non- } \\
\text { opsonized }\end{array}$ & $\begin{array}{c}\text { Human } \\
\text { monocyte } \\
\text {-derived } \\
\text { macropha } \\
\text { ges }\end{array}$ & $\begin{array}{l}\text { IF: } \mathrm{p} 47 \mathrm{phox}(20- \\
\text { 40\%) compared to } \\
\text { pathogenicity } \\
\text { island-2 mutants } \\
(\sim 70-80 \%) \text { at } 1,5, \\
20 \text { mins }\end{array}$ & $\mathrm{N} / \mathrm{A}$ & Pathogenicity island-2 & $\begin{array}{r}(308, \\
314)\end{array}$ \\
\hline $\begin{array}{c}\text { Staphylococcu } \\
\text { s aureus }\end{array}$ & + & $\begin{array}{c}\text { Faculta } \\
\text { tively } \\
\text { Anaero } \\
\text { bic }\end{array}$ & $\begin{array}{l}\text { Normal } \\
\text { human } \\
\text { serum }\end{array}$ & $\begin{array}{c}\text { Human } \\
\text { neutrophil } \\
\mathrm{s}\end{array}$ & $\begin{array}{c}\text { IF: } \\
\text { p47phox/p67phox } \\
\text { (60\%) at } 15 \text { mins; } \\
\text { gp91phox/p22phox } \\
(80 \%) \text { at } 15 \text { mins }\end{array}$ & N/A & N/A & $\begin{array}{r}(132, \\
139)\end{array}$ \\
\hline Zymosan & N/A & $\mathrm{N} / \mathrm{A}$ & $\begin{array}{c}\mathrm{IgG}, \\
\text { autologou } \\
\text { s serum, } \\
\text { normal } \\
\text { human } \\
\text { serum }\end{array}$ & $\begin{array}{c}\text { PLB-985 } \\
\text { cell line, } \\
\text { Human } \\
\text { neutrophil } \\
\text { s }\end{array}$ & $\begin{array}{l}\text { IF: YFP-tagged } \\
\text { protein } \\
\text { corresponding to } \\
\text { the C-terminus of } \\
\text { p47phox, } \\
\text { YFP-p47PRR, } \\
\text { appeared on } \\
\text { phagosomes } \sim 60 \mathrm{~s} \\
\text { after sealing and } \\
\text { internalization, } \\
\text { p47phox and } \\
\text { p67phox ( 70- } \\
90 \%) \text { at 5-20 mins, } \\
\text { g91phox }(\sim 80 \%) \\
\text { at 5-60 mins }\end{array}$ & $\begin{array}{c}\text { p47phox } \\
\text { effectively } \\
\text { phosphorylat } \\
\text { ed }\end{array}$ & N/A & $\begin{array}{l}(127, \\
309, \\
317, \\
324)\end{array}$ \\
\hline
\end{tabular}




\section{$\underline{\text { Material and Methods }}$}

\section{$\underline{\text { Neutrophil isolation }}$}

Neutrophils were isolated from blood of healthy donors using plasma-Percoll gradients as previously described (146), and in accordance with the guidelines approved by the Institutional Review Board of the University of Louisville. Microscopic evaluation of the isolated cells showed that $>95 \%$ of the cells were neutrophils. Trypan blue exclusion indicated that $>97 \%$ of cells were viable.

Bacterial strains and growth conditions.

F. alocis ATCC 38596 was cultured in brain heart infusion (BHI) broth supplemented with L-cysteine $(0.1 \%)$ and arginine $(20 \%)$ for 7 days anaerobically at $37^{\circ} \mathrm{C}$ as previously described [27, 42]. Opsonized $F$. alocis was prepared in $10 \%$ normal human serum at $37^{\circ} \mathrm{C}$ for 20 min and cultures were washed three times with PBS prior to use (Complement Technology, Tyler, Texas). For viability studies, heat killed $F$. alocis was generated by incubation at $90{ }^{\circ} \mathrm{C}$ for $60 \mathrm{~min}$. For fluorescence microscopy assays, CFSE-labeled $F$. alocis was labeled with CFSE (4 mg/mL) for 30 mins at room temperature in the dark, and the cultures were washed three times with PBS prior to use.

BacLight assay.

The combination of two DNA dyes, membrane-permeable Syto9 (stains all bacteria) and membrane-impermeable propidium iodide (PI) (stains only nonviable bacteria), was used to determine bacteria viability associated with human neutrophils as previously described (360). Human neutrophils $\left(2 \times 10^{6}\right.$ cells $\left./ \mathrm{mL}\right)$ were settled on human serum-coated coverslips, incubated in RPMI 1640 with $10 \%$ fetal bovine serum, and challenged with opsonized $F$. alocis (MOI 10) and centrifuged at $600 \mathrm{x} g$ for $4 \mathrm{~min}$ at $14^{\circ} \mathrm{C}$ to synchronize phagocytosis. Following challenge of 0.5-2-4 $\mathrm{h}$, in a cell culture incubator at $37^{\circ} \mathrm{C}$, mixed dye solutions $(5 \mu \mathrm{M}$ Syto $9,30 \mu \mathrm{M}$ PI in 0.1 MOPS (morpholinepropanesulfonic acid) $(\mathrm{pH}$ 7.2)-1 $\mathrm{mM} \mathrm{MgCl2}$ ) were added to samples. Confocal images were acquired within 30 min using a Fluoview FV1000 confocal microscope and 
analyzed by FV-10ASW software. Quantification was performed by counting the total viable and nonviable bacteria both intracellularly and extracellularly from 100 neutrophils in 3 independent experiments.

\section{Extracellular respiratory burst response.}

Human neutrophils $\left(4 \times 10^{6}\right.$ cells $\left./ \mathrm{mL}\right)$ were left unstimulated or were challenged with fMLF (300 nM; Sigma) for $5 \mathrm{~min}$ or with $F$. alocis for 5, 15, 30, 60, 90, 120, 150 min or with heatkilled $F$. alocis for 60 mins or with spent supernatant from $F$. alocis for 60 mins or with $\mathrm{PAM}_{3} \mathrm{CSK}_{4}$ (1 $\mu \mathrm{g} / \mathrm{mL}$, Invivogen) for 30 mins or with FSL-1 (100 ng/mL, Invivogen) for 30 mins at $37^{\circ} \mathrm{C}$. For neutrophil priming assays, neutrophils were pretreated with TNF- $\alpha(2 \mathrm{ng} / \mathrm{ml})$ for $10 \mathrm{~min}$ or with heat-killed $F$. alocis for 60 mins or with spent supernatant from $F$. alocis for 60 mins or with $\mathrm{PAM}_{3} \mathrm{CSK}_{4}(1 \mu \mathrm{g} / \mathrm{mL}$, Invivogen) for 30 mins or with FSL-1 (100 $\mathrm{ng} / \mathrm{mL}$, Invivogen) for $30 \mathrm{mins}$ at $37^{\circ} \mathrm{C}$, followed by stimulation with $\mathrm{fMLF}(300 \mathrm{nM})$ for $5 \mathrm{~min}$. After stimulation, the samples were centrifuged for $10 \mathrm{~min}$ at $600 \mathrm{x} g$ and $4^{\circ} \mathrm{C}$, and supernatants were collected. Superoxide anion release was measured spectrophotometrically at $550 \mathrm{~nm}$ as the superoxide dismutase-inhibitable reduction of ferricytochrome $c$ as previously described (146).

NADPH oxidase immunofluorescence microscopy.

To assess localization of NADPH oxidase subunits to $F$. alocis-infected neutrophils, we used previously established methods (127). Briefly, human neutrophils $\left(2 \times 10^{6}\right.$ cells $\left./ \mathrm{mL}\right)$ were plated onto serum-coated coverslips in RPMI 1640 with $10 \%$ fetal bovine serum and then infected with $F$. alocis at an MOI of 10:1 or with Zymosan $(2 \mathrm{mg} / \mathrm{mL})$. Phagocytosis was synchronized by centrifugation at $600 \mathrm{x} g$ at $14^{\circ} \mathrm{C}$ and after $15-30-60 \mathrm{~min}$ in a $37^{\circ} \mathrm{C}$ cell culture incubator, samples were washed with PBS, fixed in $10 \%$ formalin, permeabilized in $-20^{\circ} \mathrm{C}$ acetone/methanol solution, and then blocked in buffer containing PBS $+0.02 \%$ saponin with $10 \%$ goat serum. Cells were stained with antibodies to gp9 ${ }^{\text {phox }}$ (Santa Cruz Biotechnology, sc-130543, 1:1000) or $\mathrm{p} 22^{\text {phox }}$ (Santa Cruz Biotechnology, sc-130550, 1:500) or p6 $7^{\text {phox }}$ (BD Biosciences, $610912,1: 500)$ or $\mathrm{p} 47^{\text {phox }}$ (BD Biosciences, 610354, 1:500) or $\mathrm{p} 40^{\text {phox }}$ (Abcam, ab76158, 1:500). 
Secondary antibodies were conjugated to AlexaFluor 555 (Life Technologies, 1:500) or

AlexaFluor 647 (Life Technologies, 1:1000) and DAPI (3 $\mu \mathrm{M})$ was applied for 5 min at room temperature as a nuclear stain. Confocal images and z-stacks (1- $\mu \mathrm{m}$ thickness for each slice) were obtained using a Fluoview FV1000 confocal microscope with a $63 \mathrm{X}$ oil objective to determine NADPH oxidase component recruitment to bacterium or particulate-containing phagosomes. To quantify the enrichment of the phagosomes for each NADPH oxidase component, 100 neutrophils were counted per condition, and if $\geq 50 \%$ of the phagosome was surrounded by the component, it was considered positive recruitment.

$\underline{\text { Rac activation assay. }}$

Human neutrophils $\left(18 \times 10^{6}\right.$ cells/condition) were left unstimulated, challenged with fMLF (300 nM), or with opsonized F. alocis or with opsonized heat-killed F. alocis at an MOI of 10 for 6 min, or challenged with opsonized $F$. alocis or with opsonized heat-killed $F$. alocis at an MOI of 10 for $15 \mathrm{~min}$, or challenged with opsonized $F$. alocis or with opsonized heat-killed $F$. alocis at an MOI of 10 for 30 min, or left unstimulated or challenged with opsonized F. alocis or with opsonized heat-killed F. alocis at an MOI of 10 for $60 \mathrm{~min}$. Samples were collected, processed, and analyzed according to manufacturer's protocol (Rac 1/2/3 G-LISA activation assay, Cytoskeleton, Inc.) Briefly, following challenge, cells were washed and lysed in cell lysis buffer. Lysates were snap frozen in liquid nitrogen to ensure samples collected were GTP-bound Rac. After measuring protein concentration and equalizing the lysates, lysates were added to Rac-GTP binding 96-well plate. Lysates were bound to plate in provided binding buffer on a cold orbital microplate shaker $(200 \mathrm{rpm})$ at $4^{\circ} \mathrm{C}$ for $30 \mathrm{~min}$. Following washes, the plate was incubated with anti-Rac primary antibody on the orbital microplate shaker $(200 \mathrm{rpm})$ at room temperature for 45 min. Following washes, the plate was incubated with HRP-labeled secondary antibody on the orbital microplate shaker $(200 \mathrm{rpm})$ at room temperature for $45 \mathrm{~min}$. Following washes, the plate was incubated with HRP detection reagents at room temperature for $20 \mathrm{~min}$. Following the $20 \mathrm{~min}$ 
incubation, HRP Stop Buffer was added to each well. The signal, detecting GTP-bound Rac, was measured at $490 \mathrm{~nm}$ using a SpectraMax Soft Max Pro 5.4 spectrophotometer.

Statistical analysis.

For all the experimental conditions tested in this study, the statistical analysis used was a one-way ANOVA with the Tukey-Kramer multiple-comparison test (GraphPad Prism software, San Diego, CA, USA). Differences were considered statistically significant at the level of $\mathrm{P}<0.05$. 
$\underline{\text { Figures and Figure Legends }}$

A Brightfield

\section{Syto9}

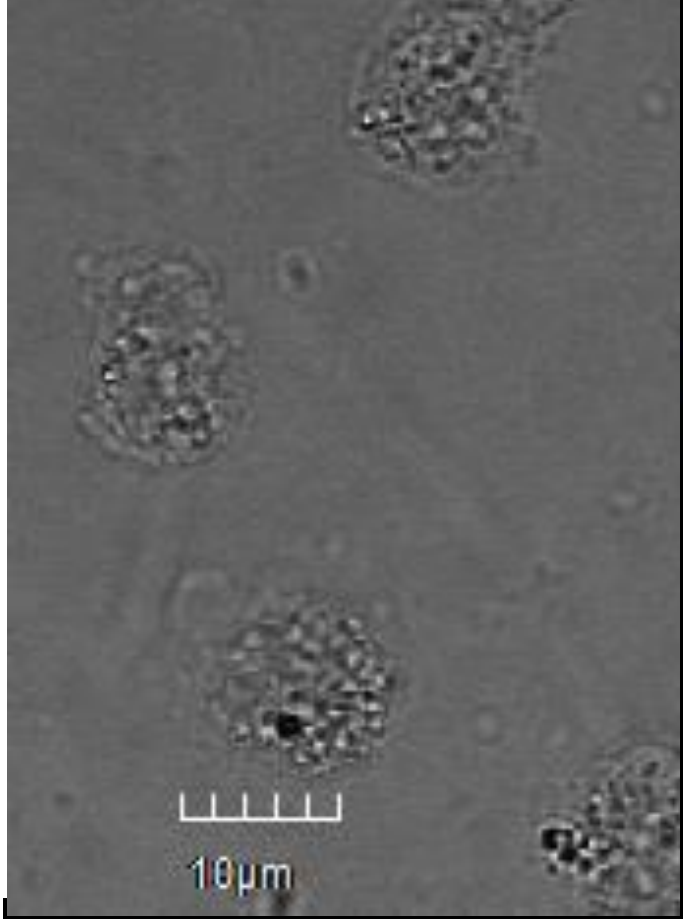

PI

Merge
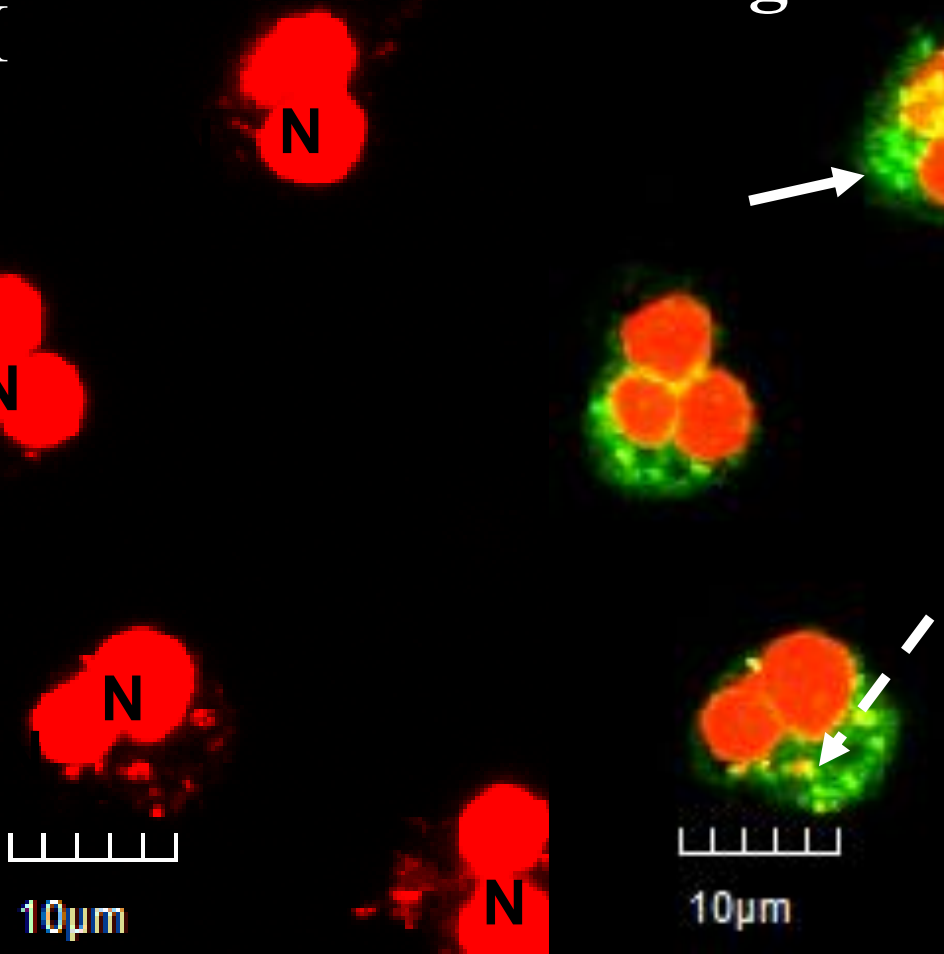

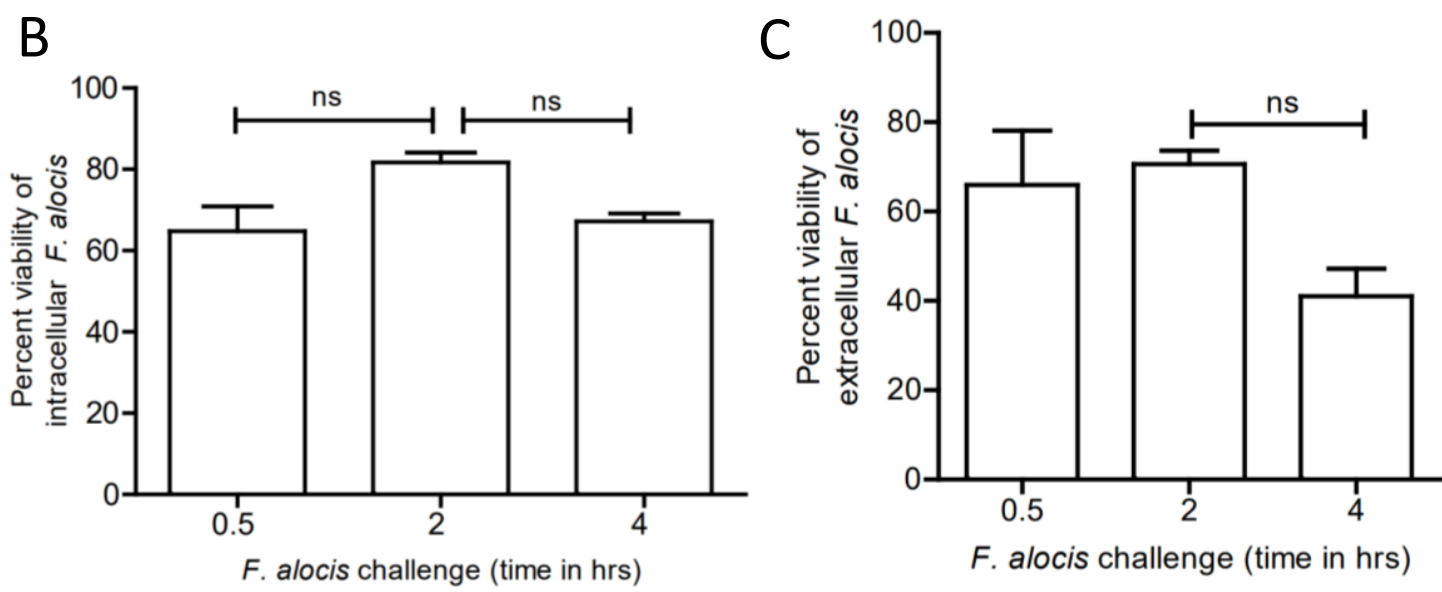

Figure 3-1. F. alocis remains viable intracellularly and extracellularly up to 4 h postneutrophil challenge.

Neutrophils were challenged with opsonized F. alocis for 0.5-2-4 h. (A) Representative confocal image of viable (green) and nonviable (red) F. alocis, which were distinguished by using the BacLight viability dyes Syto9 and PI. White solid arrow indicates viable intracellular bacteria; White dashed arrow indicates nonviable intracellular bacteria; Blue solid arrow indicates viable extracellular bacteria; Blue dashed arrow indicates nonviable extracellular bacteria. N, neutrophil nucleus. (B) Percentage of viable intracellular bacteria from 100 neutrophils from 4 independent experiments $(0.5 \mathrm{~h})$ time point and from 3 independent experiments $(2-4 \mathrm{~h})$ time points. ns, nonsignificant (C) Percentage of viable extracellular bacteria from 100 neutrophils from 3 independent experiments per time point. ns, nonsignificant. 


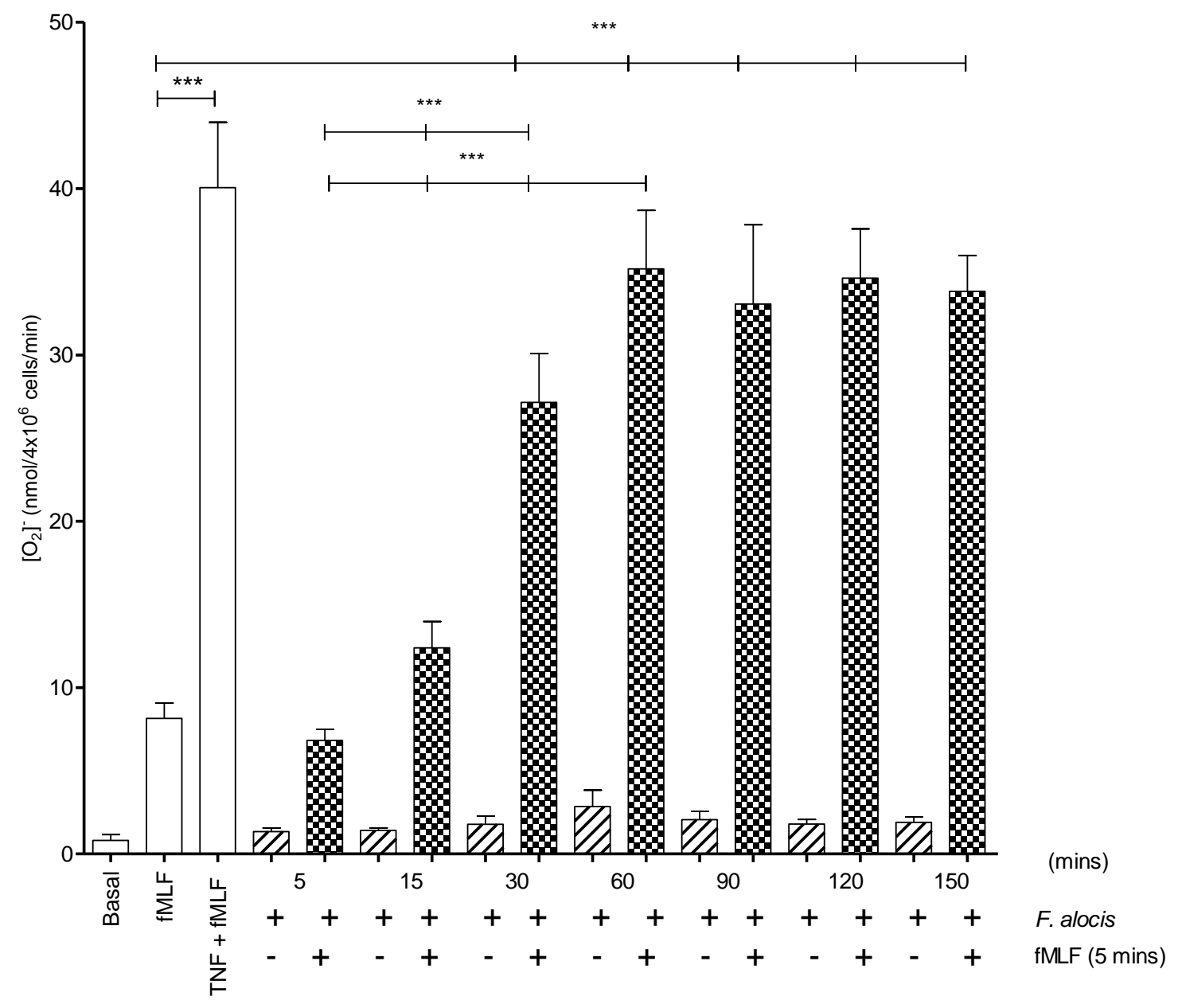

Figure 3-2. Neutrophils challenged with $F$. alocis produce minimal extracellular ROS production, but can prime neutrophils to secondary stimuli.

Neutrophils were unchallenged (Basal), stimulated with fMLF, or challenged with non-opsonized F. alocis for 5-15-30-60-90-120-150 min, or pre-treated with TNF- $\alpha$ followed by fMLF stimulation, or challenged with non-opsonized $F$. alocis for 5-15-30-60-90-120-150 min followed by fMLF stimulation. Following stimulation, extracellular production of superoxide was measured by the colorimetric reduction of ferricytochrome $c$. Data are expressed as the means \pm SEM of $\left[\mathrm{O}_{2-}\right]$ nanomoles per $4 \times 10^{6}$ cells released from 5 independent experiments. $* * * \mathrm{p}<$ 0.0001 

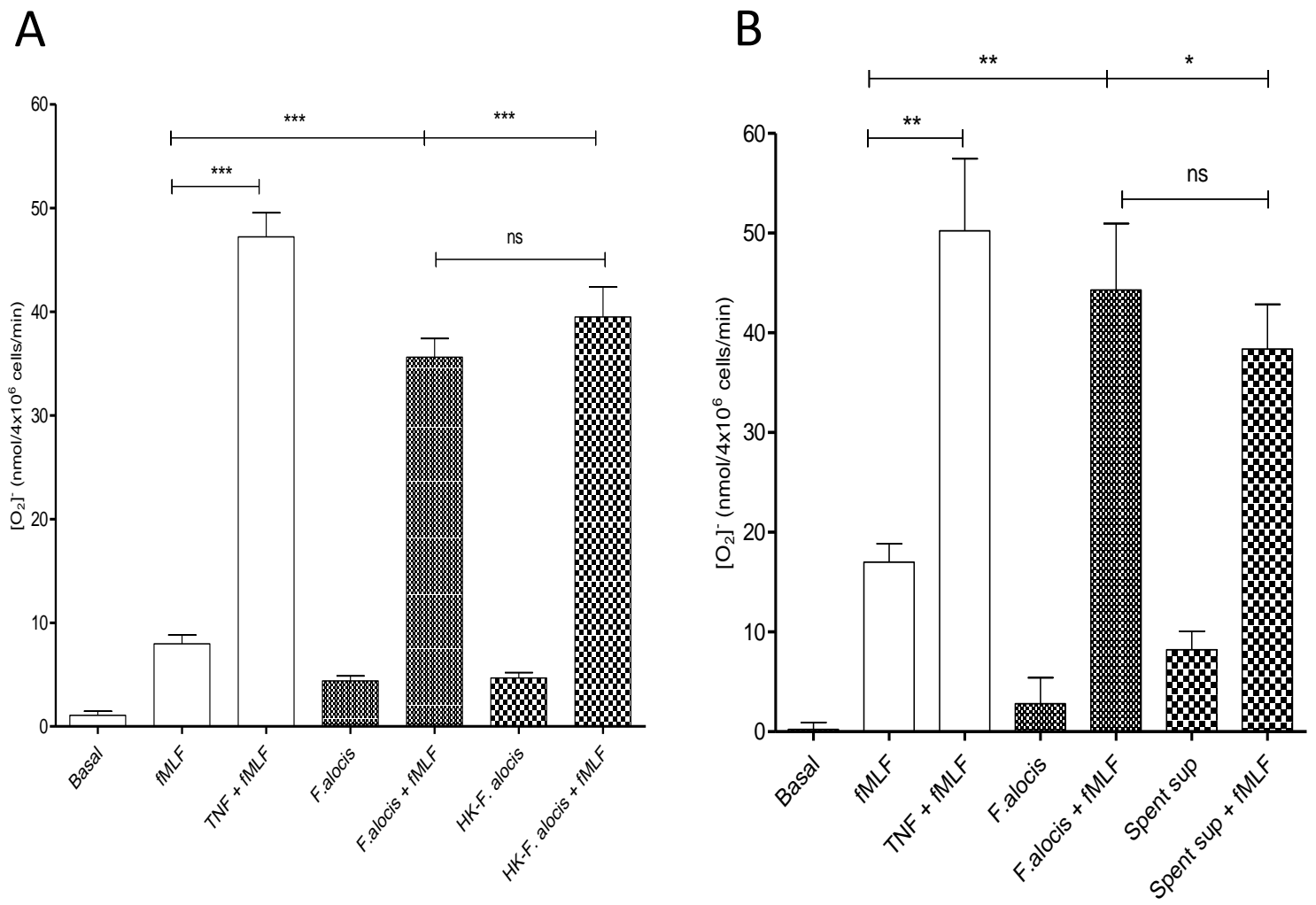

Figure 3-3. Neutrophils challenged with heat-killed $F$. alocis and $F$. alocis spent supernatant produce minimal extracellular ROS production, but can prime neutrophils to secondary stimuli.

(A) Neutrophils were unchallenged (Basal), stimulated with fMLF, or challenged with nonopsonized viable $F$. alocis for 60 min, or with non-opsonized heat-killed $F$. alocis (HK-F. alocis) for $60 \mathrm{~min}$, or pre-treated with TNF- $\alpha$ followed by fMLF stimulation, or challenged with viable $F$. alocis for 60 min followed by fMLF stimulation, or challenged with heat-killed $F$. alocis for 60 min followed by fMLF stimulation (HK-F. alocis + fMLF). Following stimulation, extracellular production of superoxide was measured by the colorimetric reduction of ferricytochrome $c$. Data are expressed as the means \pm SEM of $\left[\mathrm{O}_{2-}\right]$ nanomoles per $4 \times 10^{6}$ cells released from 6 independent experiments. $* * * \mathrm{p}<0.0001$. (B) Neutrophils were unchallenged (Basal), stimulated with fMLF, or challenged with viable $F$. alocis for $60 \mathrm{~min}$, or with $F$. alocis spent supernatants (Spent sup) for 60 min, or pre-treated with TNF- $\alpha$ followed by fMLF stimulation, or challenged with viable $F$. alocis for 60 min followed by fMLF stimulation, or 
challenged with $F$. alocis spent supernatants for 60 min followed by fMLF stimulation (Spent sup + fMLF). Following stimulation, extracellular production of superoxide was measured by the colorimetric reduction of ferricytochrome $c$. Data are expressed as the means \pm SEM of $\left[\mathrm{O}_{2-}\right]$ nanomoles per $4 \times 10^{6}$ cells released from 3 independent experiments. $* \mathrm{p}<0.05, * * \mathrm{p}<0.001$. ns, nonsignificant. 
A High Responders

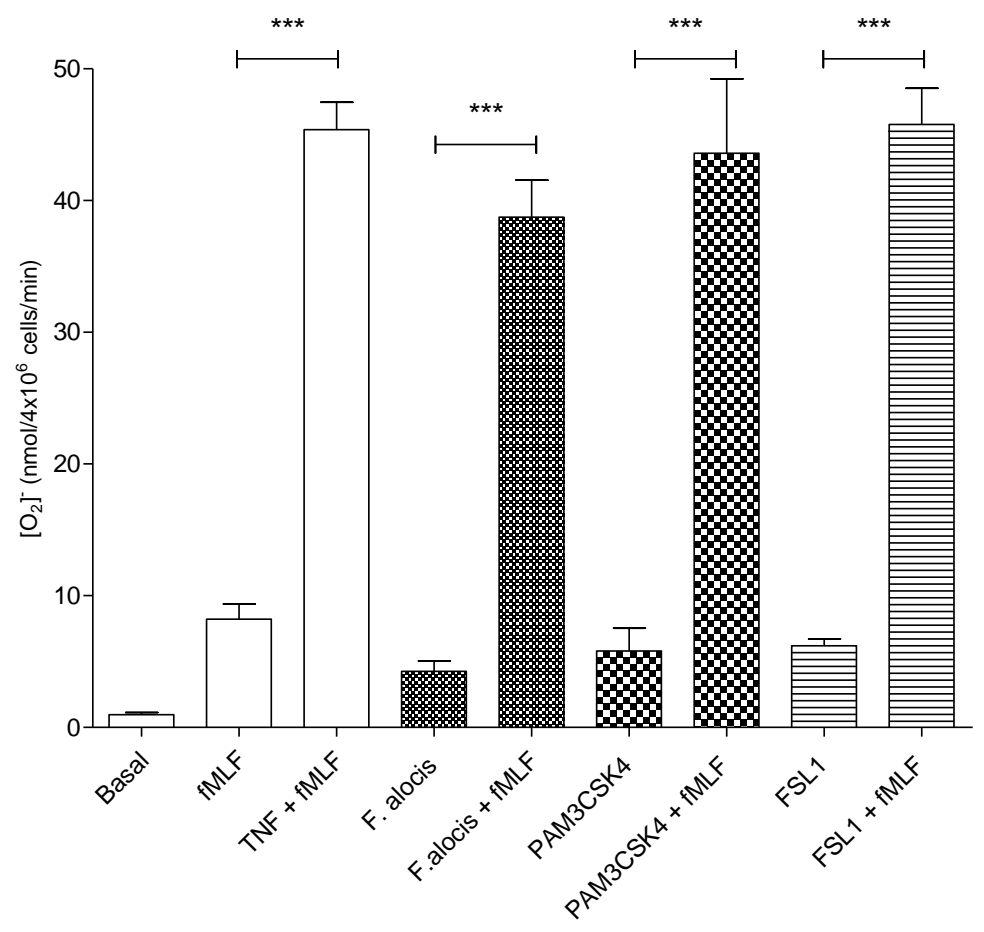

B

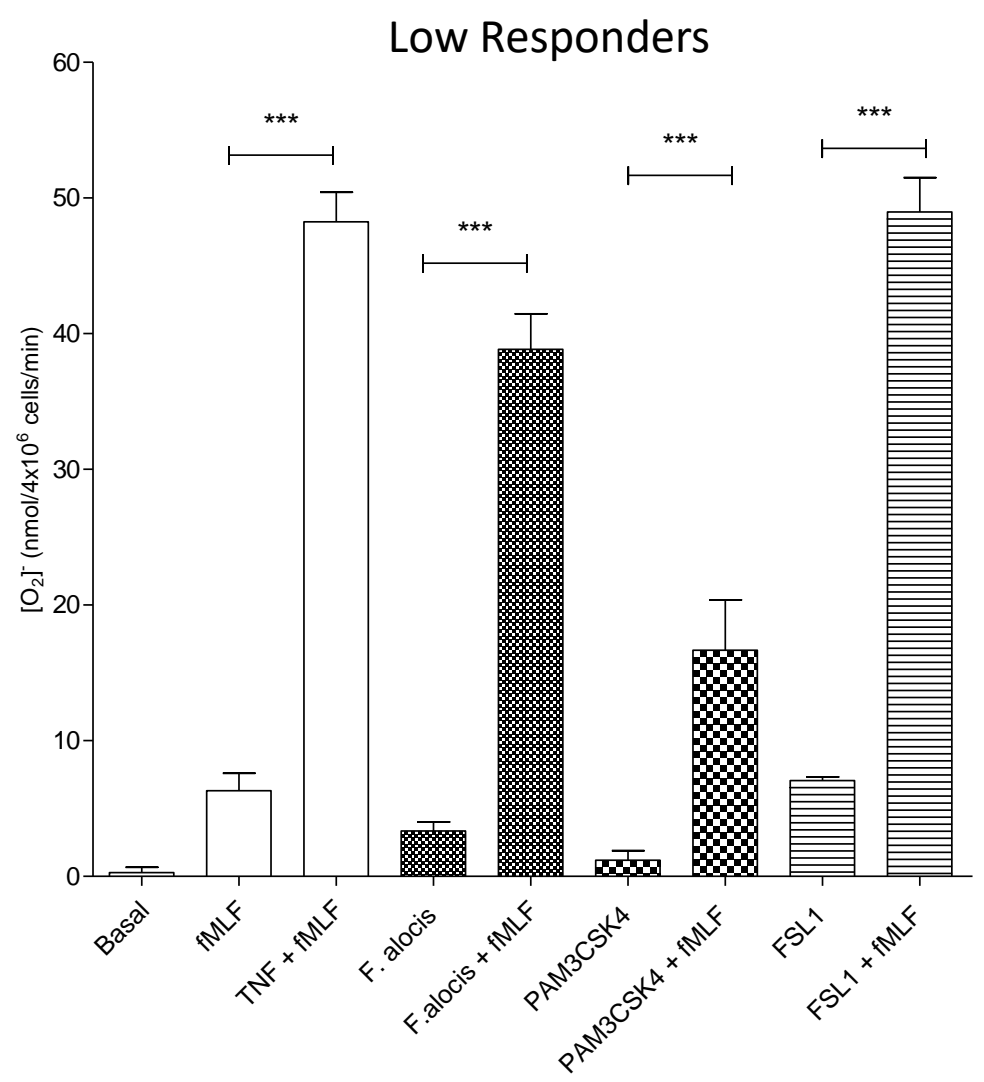


Figure 3-4. F. alocis challenge of human neutrophils may signal through TLR 2/6

Neutrophils were unchallenged (Basal), stimulated with fMLF, challenged with non-opsonized $F$. alocis or stimulated with PAM3CSK4 or stimulated with FSL-1, or pre-treated with TNF- $\alpha$, nonopsonized F. alocis, PAM3CSK4, FSL-1 followed by stimulation with fMLF. Following the different stimulation, extracellular production of superoxide was measured by the colorimetric reduction of ferricytochrome c. Data are expressed as the means $\pm \mathrm{SEM}$ of $\left[\mathrm{O}_{2^{-}}\right]$nanomoles per 4 x $10^{6}$ cells released from 5 independent experiments (Fig. 3-4 A) or from 4 independent experiments (Fig. 3-4 B). * $\mathrm{p}<0.05$. 


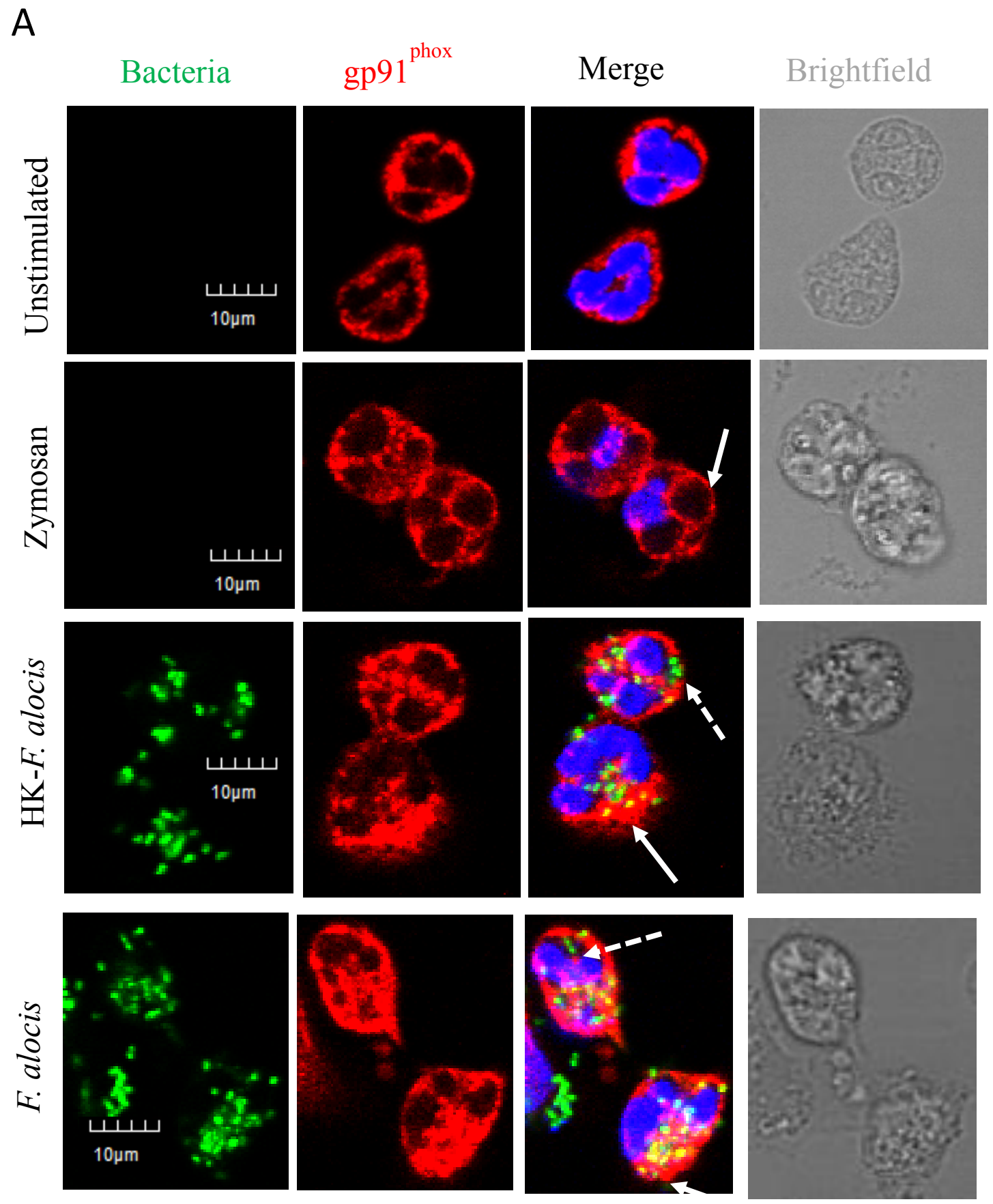


B

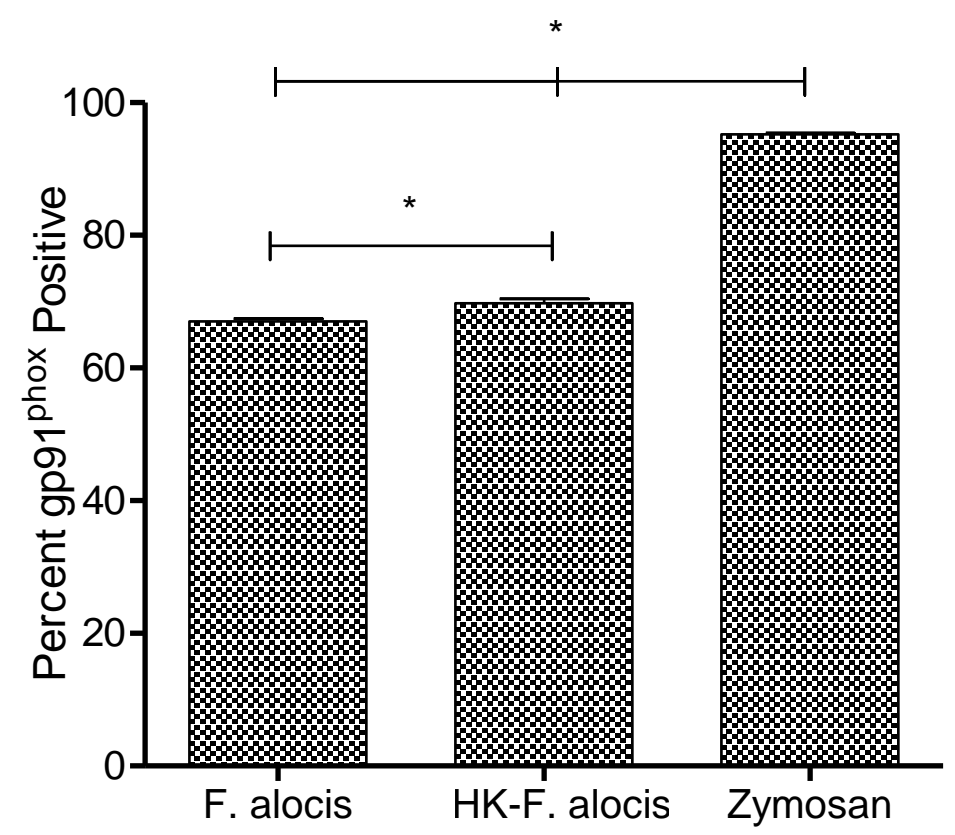

Figure 3-5. No difference in recruitment of gp91 ${ }^{\text {phox }}$ to bacteria-containing phagosomes between live and heat-killed $F$. alocis.

(A) Neutrophils were left unchallenged or challenged with opsonized Zymosan, or with opsonized CFSE-labeled heat-killed F. alocis (HK-F. alocis) or with opsonized CFSE-labeled viable F. alocis (F. alocis). Following challenge of 15 mins, cells were fixed, permeabilized, and stained with gp91 ${ }^{\text {phox }}$ antibody to visualize its recruitment to the bacterium or particulatecontaining phagosome by confocal microscopy. (B) Approximately 100 infected cells per condition were examined, and phagosomes were labeled as gp $91^{\text {phox }}$ positive if $\geq 50 \%$ of the phagosome was surrounded by gp $91^{\text {phox }}$. Solid arrows indicate gp9 $91^{\text {phox }}$ positive phagosomes and dashed arrows indicate gp9 $1^{\text {phox }}$ negative phagosomes. Data are expressed as the means \pm SEM of

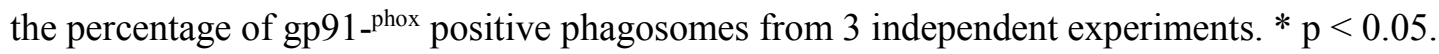




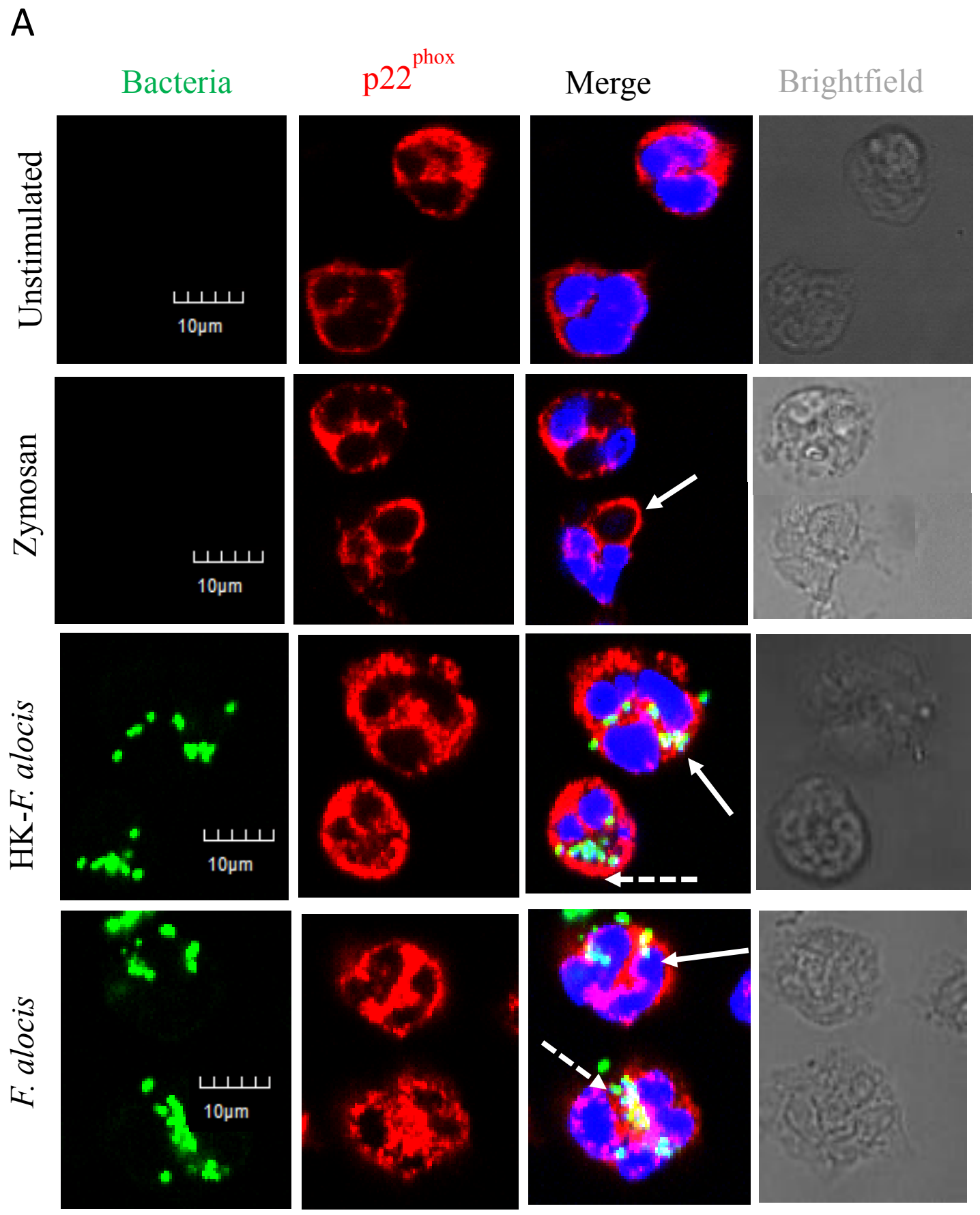




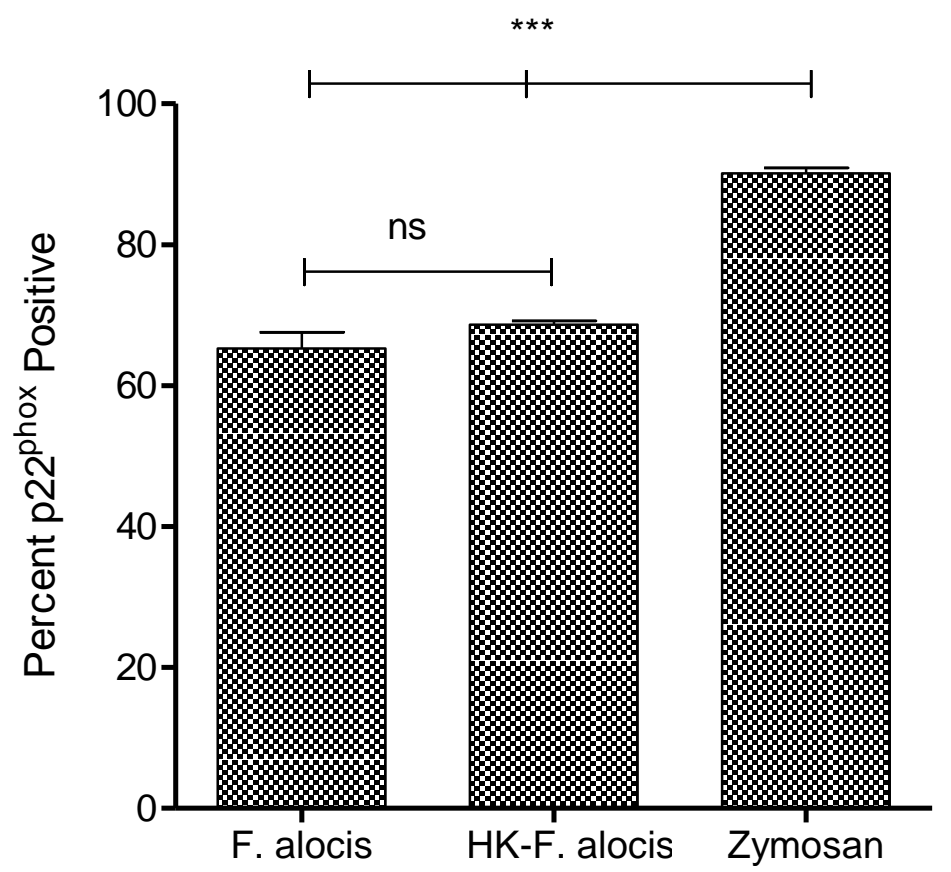

Figure 3-6. No difference in recruitment of p22 $^{\text {phox }}$ to bacteria-containing phagosomes between live and heat-killed $F$. alocis.

(A) Neutrophils were left unchallenged or challenged with Zymosan, or with CFSE-labeled heatkilled opsonized $F$. alocis (HK-F. alocis) or with CFSE-labeled viable opsonized $F$. alocis $(F$. alocis). Following challenge of 15 mins, cells were fixed, permeabilized, and stained with p22 $2^{\text {phox }}$ antibody to visualize its recruitment to the bacterium or particulate-containing phagosome by confocal microscopy. (B) Approximately 100 infected cells per condition were examined, and phagosomes were labeled as $\mathrm{p} 22^{\text {phox }}$ positive if $\geq 50 \%$ of the phagosome was surrounded by p22phox. Solid arrows indicate $\mathrm{p} 22^{\text {phox }}$ positive phagosomes and dashed arrows indicate $\mathrm{p} 22^{\text {phox }}$ negative phagosomes. Data are expressed as the means \pm SEM of the percentage of $\mathrm{p} 22^{\text {phox }}$ positive phagosomes from 3 independent experiments. ${ }^{* * *} \mathrm{p}<0.0001$. ns, nonsignificant 
A
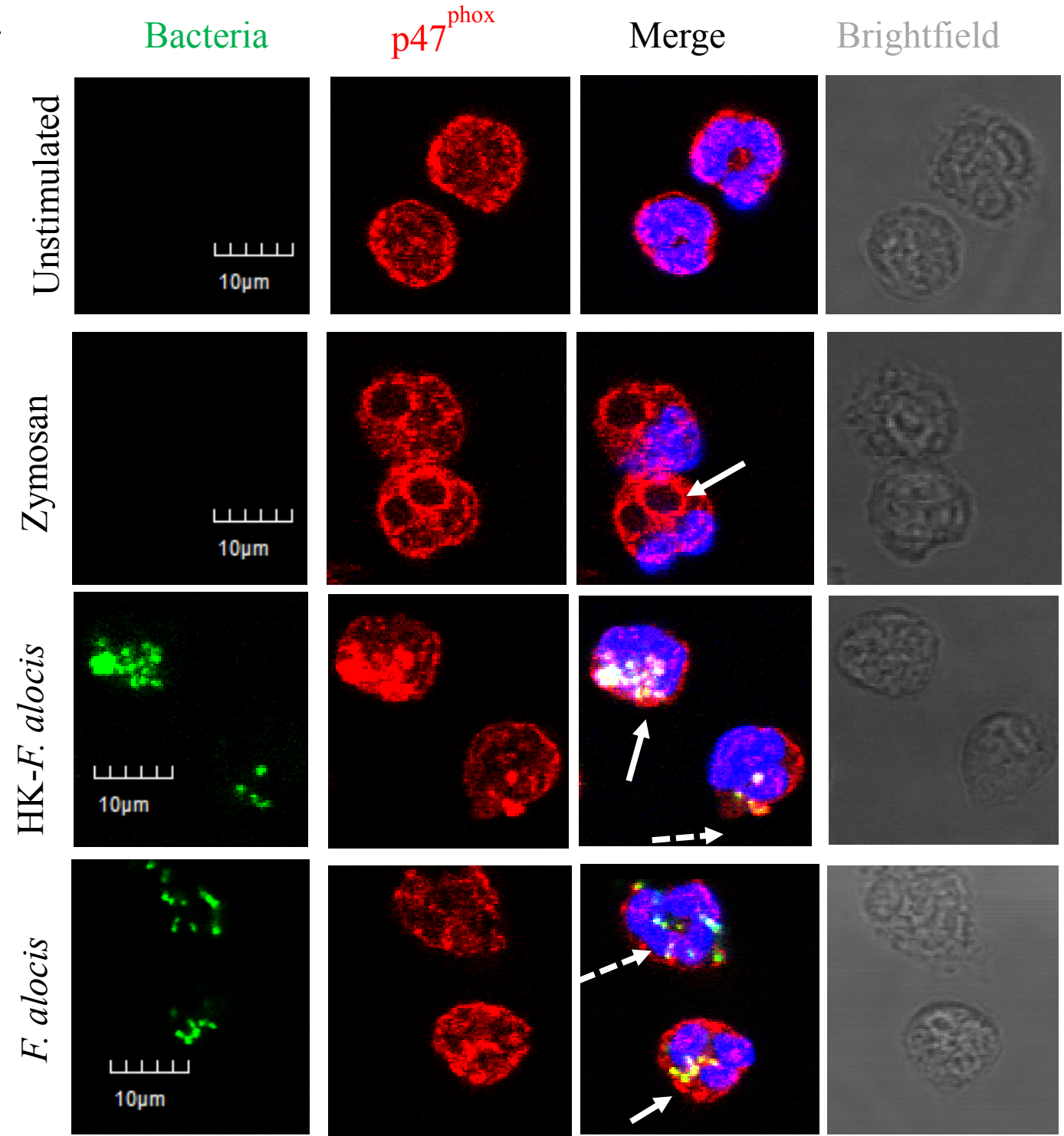
B

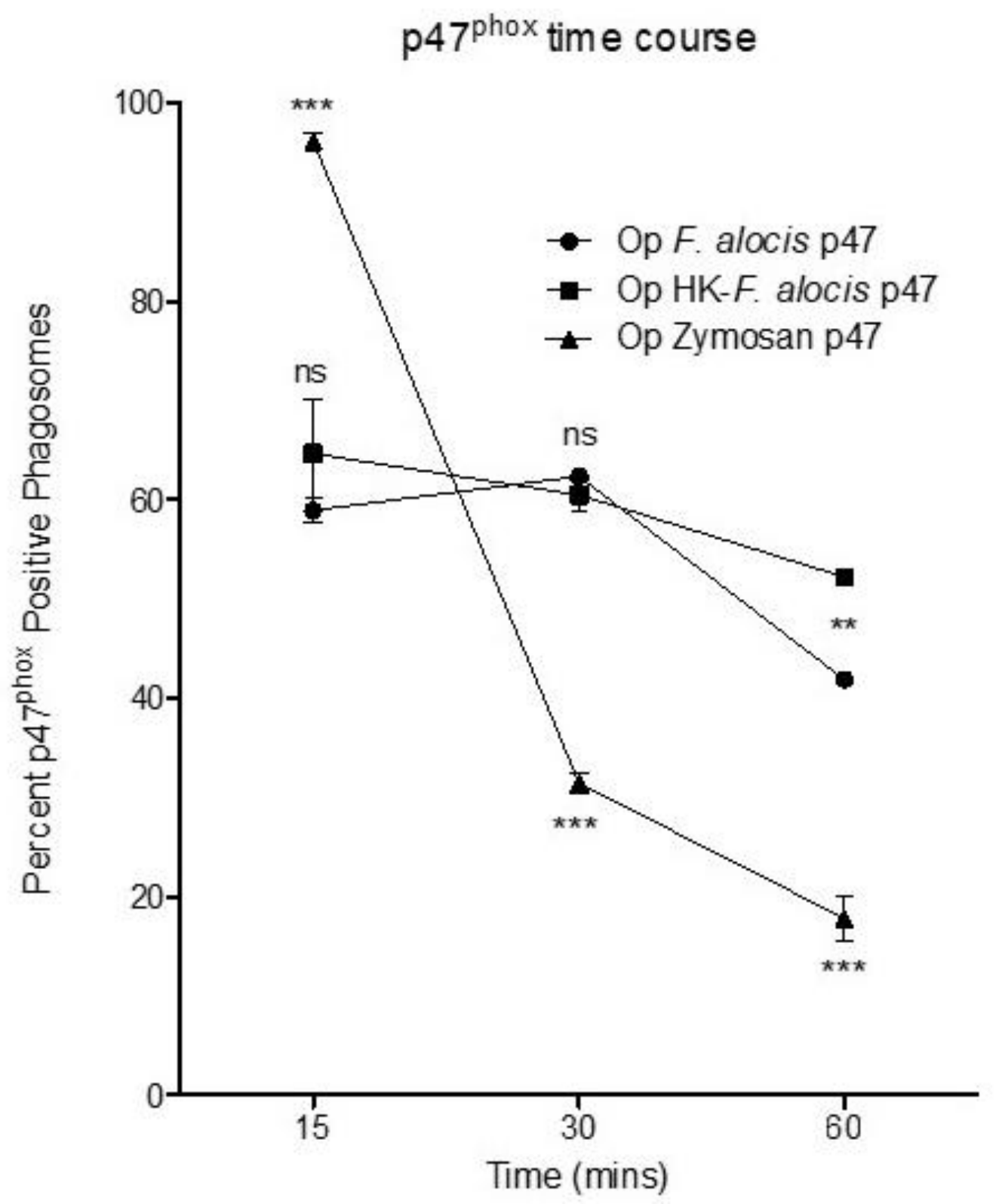

Figure 3-7. No difference in recruitment of $4^{\text {phox }}$ to bacteria-containing phagosomes between live and heat-killed $F$. alocis at 15-30 mins.

(A) Neutrophils were left unchallenged or challenged with Zymosan, or with CFSE-labeled heatkilled opsonized $F$. alocis (HK-F. alocis) or with CFSE-labeled viable opsonized $F$. alocis $(F$. alocis). Following challenge of 15 mins, cells were fixed, permeabilized, and stained with $\mathrm{p} 47^{\text {phox }}$ antibody to visualize its recruitment to the bacterium or particulate-containing phagosome by 
confocal microscopy. (B) At 15 mins post $F$. alocis challenge, approximately 100 infected cells per condition were examined, and phagosomes were labeled as $\mathrm{p} 47^{\text {phox }}$ positive if $\geq 50 \%$ of the phagosome was surrounded by $\mathrm{p} 47^{\text {phox }}$. Solid arrows indicate $\mathrm{p} 47^{\text {phox }}$ positive phagosomes and dashed arrows indicate $\mathrm{p} 47^{\text {phox }}$ negative phagosomes. Data are expressed as the means $\pm \mathrm{SEM}$ of the percentage of $\mathrm{p} 47^{\text {phox }}$ positive phagosomes from 3 independent experiments. ${ }^{* *} \mathrm{p}<0.01, * * * \mathrm{p}$ $<0.0001$. ns, nonsignificant. At 30 mins post $F$. alocis challenge, approximately 100 infected cells per condition were examined, and phagosomes were labeled as $\mathrm{p} 47^{\text {phox }}$ positive if $\geq 50 \%$ of the phagosome was surrounded by $4^{77^{\text {phox }}}$. Data are expressed as the means $\pm \mathrm{SEM}$ of the percentage of $\mathrm{p} 47^{\text {phox }}$ positive phagosomes from 3 independent experiments. $* * * \mathrm{p}<0.0001$. At 60 mins post $F$. alocis challenge, approximately 100 infected cells per condition were examined, and phagosomes were labeled as $\mathrm{p} 47^{\text {phox }}$ positive if $\geq 50 \%$ of the phagosome was surrounded by $\mathrm{p} 47^{\text {phox }}$. Data are expressed as the means \pm SEM of the percentage of $\mathrm{p} 47^{\text {phox }}$ positive phagosomes from 3 independent experiments. ${ }^{* *} \mathrm{p}<0.001,{ }^{* * *} \mathrm{p}<0.0001$. 
A

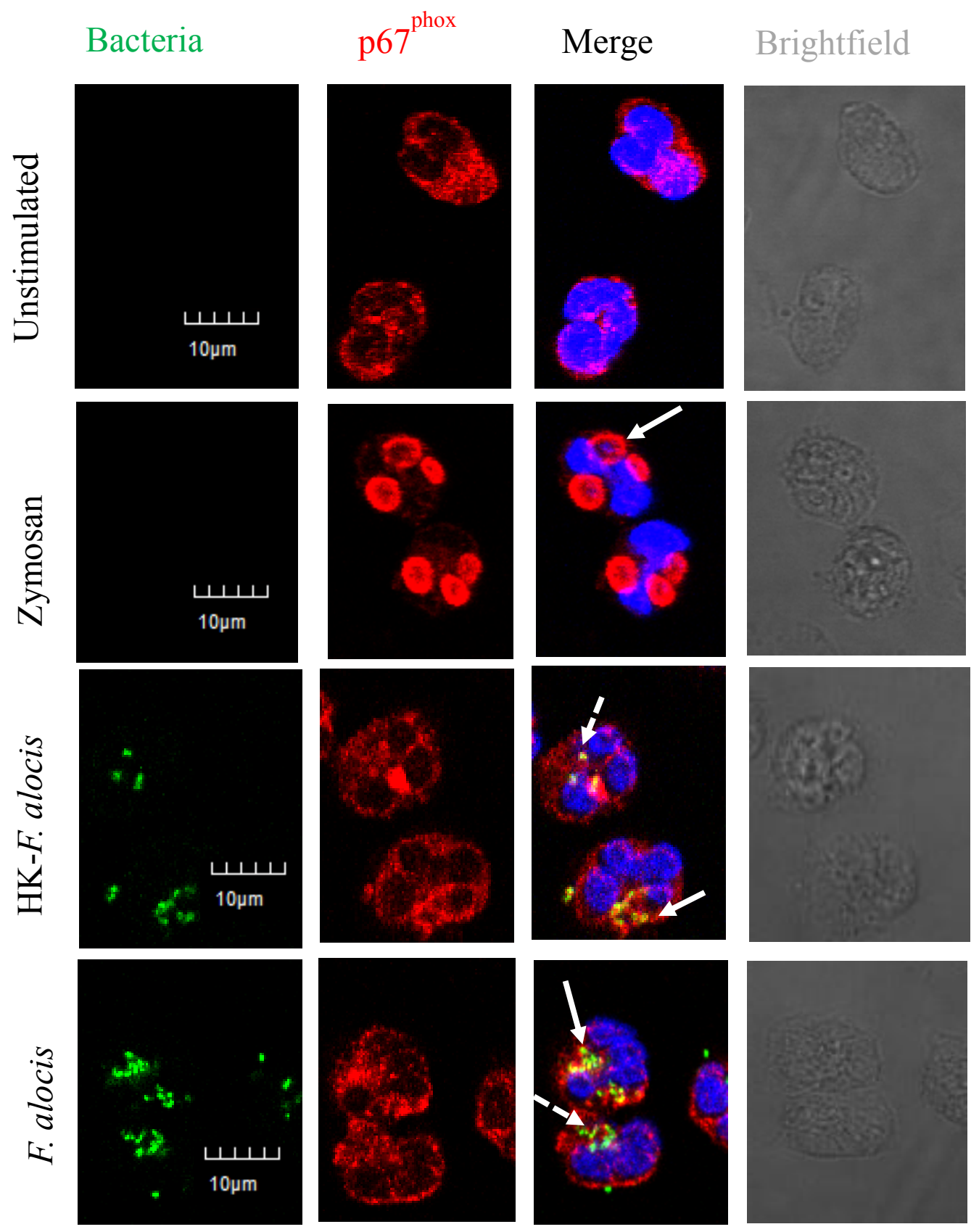




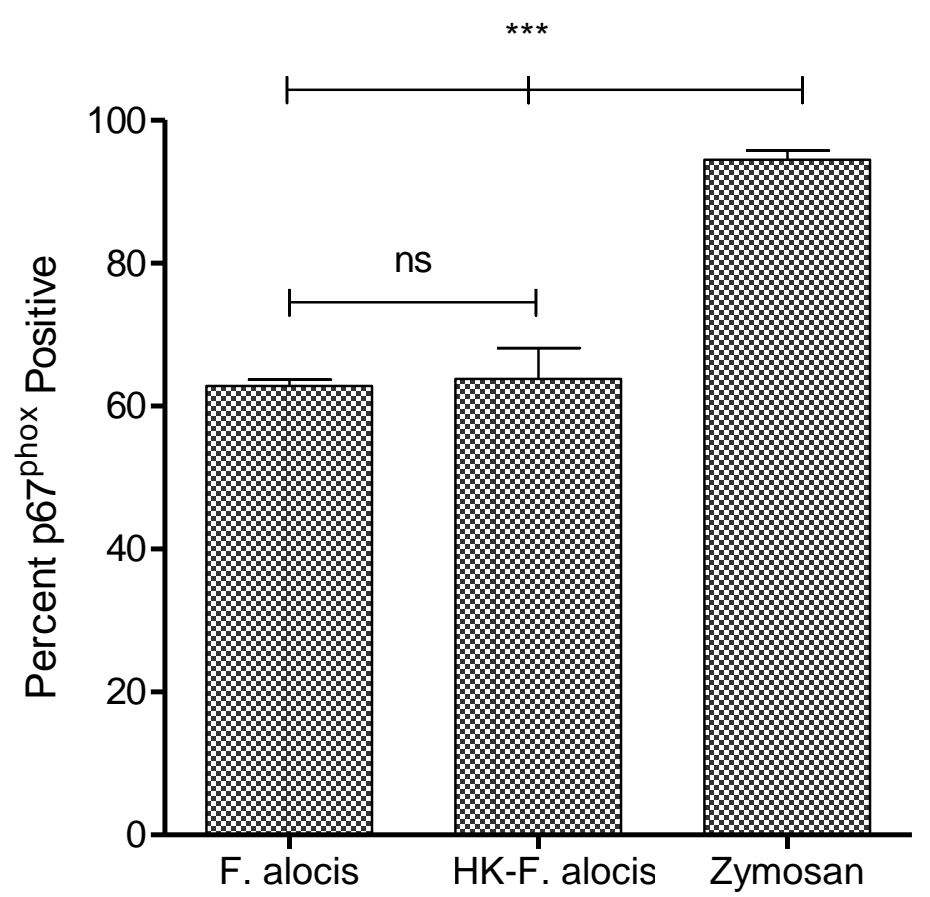

Figure 3-8. No difference in recruitment of p67 $^{\text {phox }}$ to bacteria-containing phagosomes between live and heat-killed $F$. alocis.

(A) Neutrophils were left unchallenged or challenged with Zymosan, or with CFSE-labeled heatkilled opsonized $F$. alocis (HK-F. alocis) or with CFSE-labeled viable opsonized $F$. alocis $(F$. alocis). Following challenge of 15 mins, cells were fixed, permeabilized, and stained with p6 $7^{\text {phox }}$ antibody to visualize its recruitment to the bacterium or particulate-containing phagosome by confocal microscopy. (B) Approximately 100 infected cells per condition were examined, and phagosomes were labeled as $\mathrm{p} 67^{\text {phox }}$ positive if $\geq 50 \%$ of the phagosome was surrounded by p6 $7^{\text {phox }}$. Solid arrows indicate $\mathrm{p} 67^{\text {phox }}$ positive phagosomes and dashed arrows indicate p6 $7^{\text {phox }}$ negative phagosomes. Data are expressed as the means \pm SEM of the percentage of $p 67^{\text {phox }}$ positive phagosomes from 3 independent experiments. ${ }^{* * *} \mathrm{p}<0.0001$. ns, nonsignificant. 


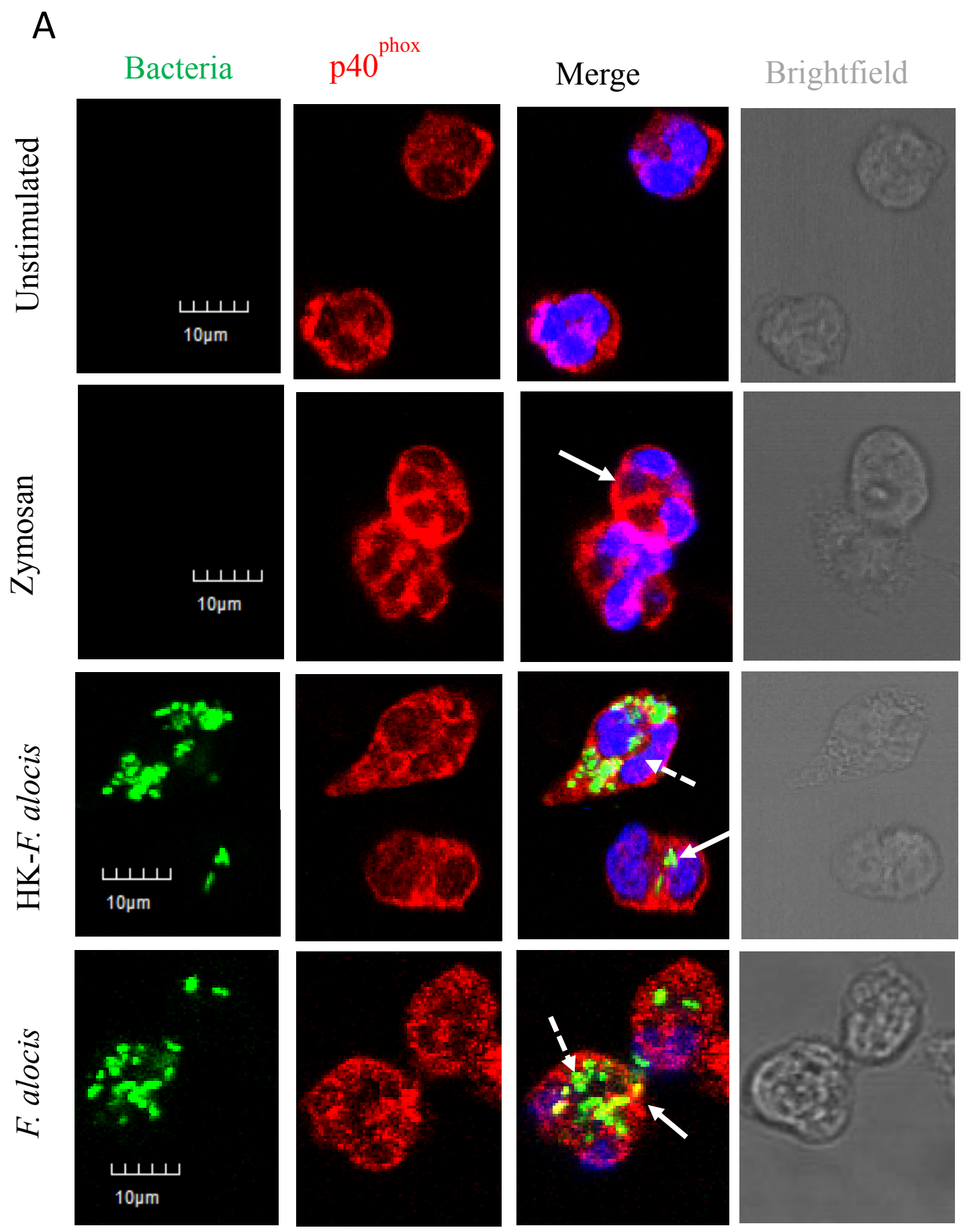


B

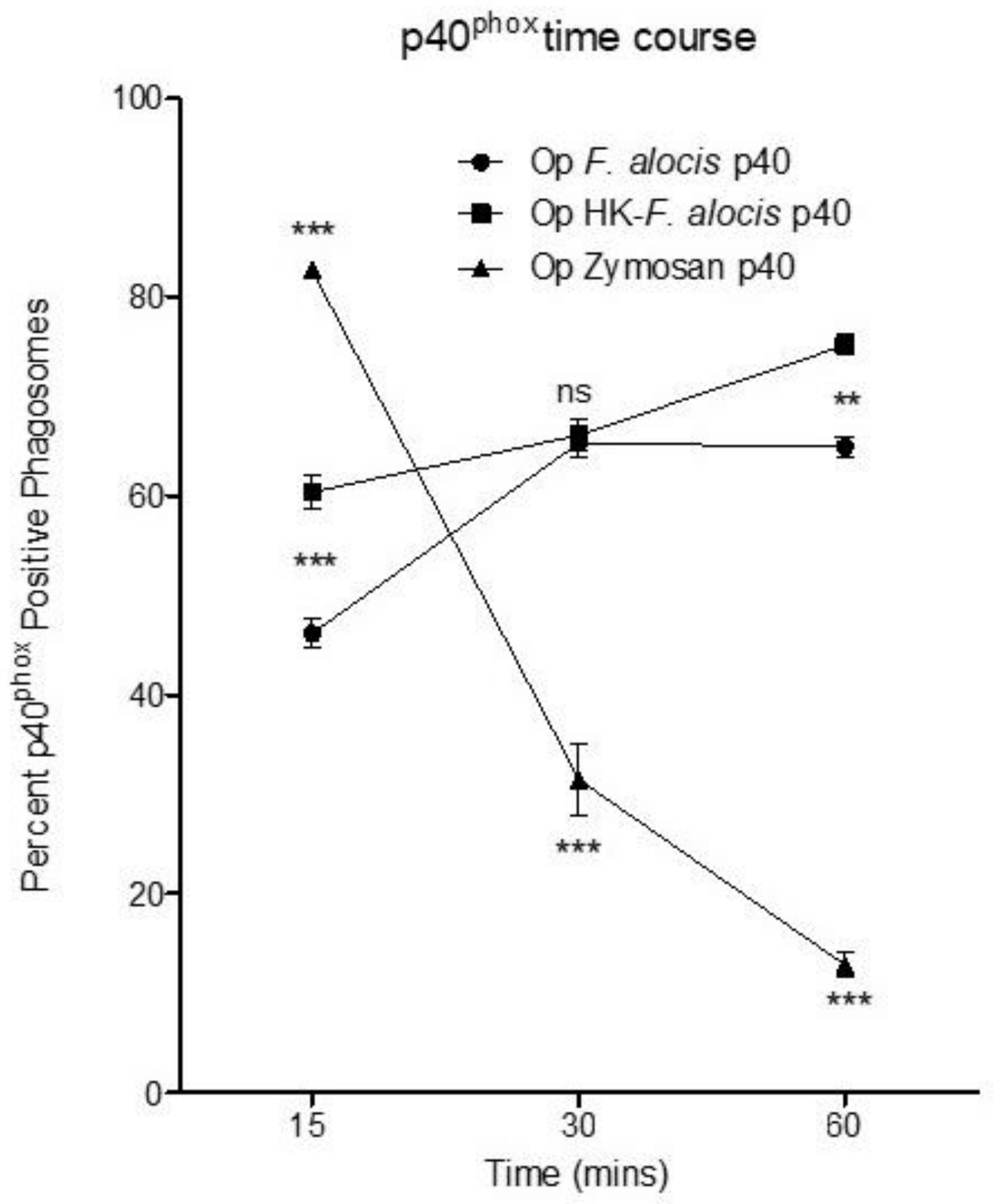

Figure 3-9. Impaired recruitment of $\mathrm{p}^{\mathrm{phox}}$ to viable $F$. alocis-containing phagosomes at 15 and 60 mins.

(A) Neutrophils were left unchallenged or challenged with Zymosan, or with CFSE-labeled heatkilled opsonized $F$. alocis (HK-F. alocis) or with CFSE-labeled viable opsonized $F$. alocis $(F$. 
alocis). Following challenge of 15 mins, cells were fixed, permeabilized, and stained with $\mathrm{p} 40^{\text {phox }}$ antibody to visualize its recruitment to the bacterium or particulate-containing phagosome by confocal microscopy. (B) At 15 mins post $F$. alocis challenge, approximately 100 infected cells per condition were examined, and phagosomes were labeled as $\mathrm{p} 40^{\text {phox }}$ positive if $\geq 50 \%$ of the phagosome was surrounded by $\mathrm{p} 40^{\text {phox }}$. Solid arrows indicate $\mathrm{p} 40$ phox positive phagosomes and dashed arrows indicate $\mathrm{p} 40^{\text {phox }}$ negative phagosomes. Data are expressed as the means $\pm \mathrm{SEM}$ of the percentage of $\mathrm{p} 40^{\text {phox }}$ positive phagosomes from 3 independent experiments. $* * * p<0.0001$. At 30 mins post $F$. alocis challenge, approximately 100 infected cells per condition were examined, and phagosomes were labeled as $\mathrm{p} 40^{\text {phox }}$ positive if $\geq 50 \%$ of the phagosome was surrounded by $\mathrm{p} 40^{\text {phox }}$. Data are expressed as the means \pm SEM of the percentage of $\mathrm{p} 40^{\text {phox }}$ positive phagosomes from 3 independent experiments. $* * * \mathrm{p}<0.0001$. At 60 mins post $F$. alocis challenge, approximately 100 infected cells per condition were examined, and phagosomes were labeled as $\mathrm{p} 40^{\text {phox }}$ positive if $\geq 50 \%$ of the phagosome was surrounded by $\mathrm{p} 40^{\text {phox }}$. Data are expressed as the means \pm SEM of the percentage of $\mathrm{p} 40^{\text {phox }}$ positive phagosomes from 3 independent experiments. ${ }^{* *} \mathrm{p}<0.001, * * * \mathrm{p}<0.0001$. 


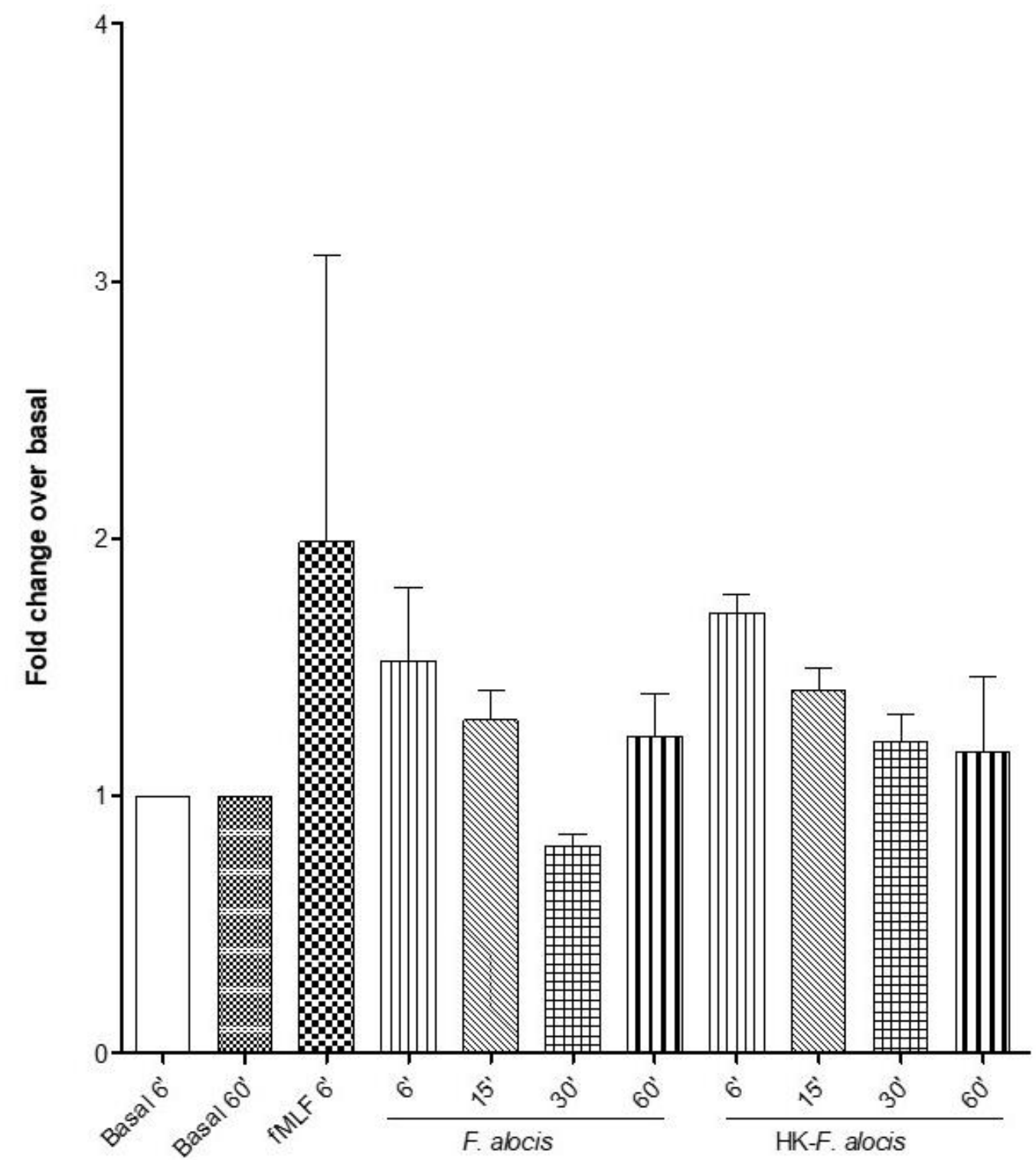

Figure 3-10. Rac activation by neutrophils challenged with $F$. alocis is impaired in viable bacteria at 30 mins.

Neutrophils were left unchallenged (Basal), stimulated with fMLF or challenged with opsonized viable $F$. alocis (F. alocis) or challenged with opsonized heat-killed $F$. alocis (HK- F. alocis) for 6-15-30-60 mins. Following stimulation, cell lysates were collected, plated with a binding buffer, washed, incubated with anti-Rac primary antibody, washed, incubated with HRP secondary antibody, incubated with HRP detection reagent and then the reaction was stopped using HRP 
Stop Solution. Conditions were read at $490 \mathrm{~nm}$ on a spectrophotometer. Data are expressed as fold change over basal of absorbance values for Rac activation from 2 independent experiments. 


\section{CHAPTER 4}

\section{FILIFACTOR ALOCIS MANIPULATES HUMAN NEUTROPHILS AFFECTING THEIR ABILITY TO INDUCE NEUTROPHIL EXTRACELLULAR TRAPS (NETs)}

\section{$\underline{\text { Introduction }}$}

Neutrophils are phagocytic polymorphonuclear leukocytes that function as the principal innate immune cell recruited to sites of infection or inflammation in the periodontal pocket. They contribute to the maintenance of periodontal health by protecting the tissue against bacterial infection through employing a variety of anti-microbial killing mechanisms (198). One such mechanism of killing is neutrophil extracellular trap (NET) formation. Although NETs were first described by Brinkmann and Zychlinsky in 2004, much work has been done since their discovery, but questions still remain on their composition, roles, regulation and contribution to diseases (165). Additionally, NETs studies remain controversial, especially in whether their effects are beneficial or detrimental to the host in the presence of infection $(165,168,361,362)$. It is essential to strictly regulate NET formation in a time and dose-dependent fashion to ensure production and clearance processes occur when it is most beneficial to the host (168).

In NET formation, neutrophils extrude their DNA extracellularly, which are decorated with antimicrobial granular proteins, like myeloperoxidase, neutrophil elastase, and histones. This process can occur in viable or dying neutrophils and is effective in trapping bacteria due to its electrostatic charge interactions and in killing due to its ability to produce a localized high concentration of antimicrobial peptides in the presence of a wide variety of stimuli $(154,157$, $168,363,364)$. Depending on the stimuli used to activate neutrophils, activation of the NADPH 
oxidase and production of intracellular ROS may or may not be required for NET formation (157, 197, 364-366).

NETs have been discovered in the gingival epithelium and can be attributed to a first response to periodontal bacteria presence by the host $(197,367)$. In the context of periodontal disease, both excessive and ineffective NET production have been associated with development of the disease (149). Many oral bacteria can produce DNase, which serves to degrade DNA, and may provide a means to escape trapping and killing by NETs $(151,197,368-372)$. However, crevicular exudate outflow may inhibit optimal functioning of the bacterial DNases, and work in concert with NETs to clear pathogens from the oral cavity and prevent development of periodontitis (197).

In this chapter, we show that $F$. alocis fails to induce NET formation, but may be able to manipulate and prevent neutrophils from forming NETs once they have been exposed to a pharmacological or bacterial stimulus that can induce NETs. These results suggest that $F$. alocis may produce a secreted factor, have an active DNA-degrading enzyme, or employ an undetermined virulence mechanism that allows for its ability to control neutrophils and prevent induction of NETs.

$\underline{\text { Results }}$ F. alocis challenge of human neutrophils fails to induce NETs across a time course.

Since NETs have implications in periodontal disease, using in vitro studies, we sought to determine if $F$. alocis, a periodontal pathogen, is capable of inducing NET formation in human neutrophils, the primary cells recruited to the gingival epithelium $(149,363,373)$. We hypothesized that $F$. alocis will modulate human neutrophil production of NETs and the subsequent response of bactericidal proteins in order to evade killing.

Our preliminary observations showed minimal intracellular ROS production when neutrophils were challenged with $F$. alocis. This could indicate that if NETs were formed in the 
context of $F$. alocis infection this may occur in an ROS-independent manner. However, using confocal immunofluorescence, we discovered on an initial screening that neutrophils challenged with serum-opsonized (Op) F. alocis for 1-4-20 h did not induce NET formation compared with PMA or S. aureus, two well-established pharmacological and bacterial inducers of NET formation (data not shown).

As it has been demonstrated that NETs can be induced at an early or late time point depending on the stimulus $(157,363)$, we performed another time course experiment challenging neutrophils with Op-F. alocis for 15-30-60-90-180 min to determine if $F$. alocis induced NETs early on upon challenge. Positive NET formation was determined by confocal microscopy, staining the neutrophil chromatin with DAPI and its colocalization with a known granule component, MPO; and using PMA as a known NET inducer (Fig. 4-1A). Our data showed that $F$. alocis did not induce NETs at any of the early time points tested (Fig. 4-1 A). Quantification of the confocal images, using the approach described by Zychlinsky et al. (374), showed that $F$. alocis induced less than 5\% of NETs, independent of the infection time (Fig. 4-1B). For a stimulus to be considered an inducer of NET formation, $\geq 10 \%$ NETs need to be formed (168). Hence, F. alocis induced minimal NET formation.

Due to previous studies suggesting the negative impacts serum and complement could have on NET induction $(364,375)$, we sought to rule out the impacts serum may play on the lack of NETs observed with $F$. alocis challenge by using non-opsonized F. alocis (Fig. 4-2 A). Additionally, in the context of the oral cavity, host-derived serum will be present, therefore testing opsonized bacteria is more physiologically relevant. We saw that similarly to the opsonized bacterium, the non-opsonized bacterium did not induce NET formation at any of the time points tested (Fig. 4-2 B), which suggests serum opsonization does not impact our conclusions that $F$. alocis is not an inducer of NETs.

Since preliminary data from our laboratory has shown that heat-killed F. alocis was able to induce a robust intracellular respiratory burst response compared to the live organisms, we 
sought to determine if this difference would be observed with NET formation. However, in our time course induction of NETs with heat-killed $F$. alocis, the bacterium did not induce NET formation (Fig. 4-3 A, B). This demonstrates that viability is not playing a role in the induction of NETs by F. alocis challenge.

$\underline{F}$ alocis challenge of neutrophils produces minimal levels of extracellular NE.

Several studies have highlighted the importance of NET component neutrophil elastase (NE) to the effective formation and function of NETs $(170,376,377)$. Therefore, using a kit to detect extracellular release of NE, we sought to determine if its presence was found with nonopsonized and opsonized $F$. alocis challenge of neutrophils. We performed a time course study with basal, and $F$. alocis -challenged neutrophils and detected the levels of NE released extracellularly at 15-30-60-90 min. Basal neutrophils and non-opsonized and opsonized F. alocis challenged neutrophils released NE to similar levels, which was expected based on the previous observations of minimal presence of NETs (Fig. 4-4 A). For the later time points of 60-90-120 min, PMA was included as a positive control, which showed higher levels of NE compared to the bacteria challenge (Fig. 4-4 B). This data indicates that F. alocis challenge of neutrophils does not produce a significant amount of extracellular NE, which is critical for effective NET formation, which may help explain minimal NET induction by F. alocis that is observed by immunofluorescence microscopy. Priming neutrophils before bacterial challenge does not impact NET formation.

Previous studies have reported primed neutrophils can undergo NET formation, so we wanted to determine if NETs would be induced if we primed neutrophils with TNF- $\alpha$ for 10 min and then challenged with non-opsonized or opsonized $F$. alocis for $180 \mathrm{~min}$. There was no significant induction of NETs following priming and then bacterial challenge with $F$. alocis compared with F. alocis alone (Fig. 4-5). This suggests that priming neutrophils with TNF does not seem to pre-activate/dispose neutrophils to form NETs in response to $F$. alocis challenge. $\underline{\text { S. gordonii and P. stomatis induce NET formation in an MOI-dependent manner. }}$ 
Interestingly, F. alocis, a pathogen known to be an indicator of oral disease presence, did not induce NET formation by human neutrophils. In order to determine if $F$. alocis is unique in its lack of NET induction, other species common to the oral cavity, Streptococcus gordonii and Peptoanaerobacter stomatis, were used to challenge neutrophils and determine if NETs were produced. No significant NET formation was observed with either S. gordonii or P. stomatis between 5 up to 90 min; only by 180 min both oral bacteria showed NET induction (data not shown). Using immunofluorescence microscopy, we were able to determine that after $180 \mathrm{~min}$ bacterial challenge at MOI 10 both $S$. gordonii and P. stomatis induced NET formation significantly higher than non-opsonized and opsonized F. alocis (Fig. 4-6 A, B). Additionally, $S$. gordonii is able to induce a significantly more robust NET response than P. stomatis (Fig. 4-6 B). Challenging neutrophils with an increasing MOI of bacteria can result in NET induction, as observed by previous studies $(378,379)$. Hence, we challenged neutrophils with all three oral bacteria, F. alocis, S. gordonii and P. stomatis at higher bacteria loads of MOI 50 and 100 (Fig. 46A). Our data showed that S. gordonii induced NETs in a concentration-dependent manner, however P. stomatis NET induction peaked at MOI 50; but was not significantly different at MOI 100 (Fig.4-6 D). In contrast, challenging neutrophils with F. alocis at MOIs of 50 and 100, did not induce NET formation (Fig. 4-6 C, D). Our results indicate that F. alocis does not induce NETs in an MOI-dependent manner. In summary, these results led us to the conclusion that $F$. alocis may be unique to the oral community in its lack of NET induction, independent of MOI and time.

In order to more closely study $F$. alocis in the context of an oral community, we performed 180 min time point co-infection studies with non-opsonized and opsonized $F$. alocis and S. gordonii or P. stomatis. In comparison with S. gordonii alone at $180 \mathrm{~min}$, we observed that there was no significant increase or decrease in NET formation in the presence of $F$. alocis challenge (Fig.4-7 A). Similarly, in comparison with P. stomatis alone at $180 \mathrm{~min}$, we observed that there was no significant increase or decrease in NET formation in the presence of $F$. alocis 
challenge (Fig. 4-7 B). This led us to determine that in a co-infection setting, F. alocis cannot inhibit or exacerbate NETs formed by $S$. gordonii or $P$. stomatis, bacterial stimuli that we determined can effectively induce NETs.

We next sought to determine if $F$. alocis can actively degrade pre-formed NETs. To accomplish this, we challenged neutrophils with $S$. gordonii or $P$. stomatis, which were already determined to induce NETs, for 90 min and then introduced non-opsonized and opsonized $F$. alocis for 90 min. We saw no significant increase or decrease in NET formation (Fig. 8). Based on this data, we can conclude that once NETs are formed, F. alocis does not have the capacity to degrade them or inhibit their formation.

Based on the results obtained in Figures 4-7 and 4-8, we thought it was necessary to determine if $F$. alocis has the capabilities of preventing or inhibiting NET formation. We decided pre-treat neutrophils with $F$. alocis followed by the well-established, non-physiological, positive stimulus for NET formation, PMA. In this experiment, we pre-treated neutrophils with nonopsonized F. alocis for 30-60 min before PMA exposure (180 $\mathrm{min}$ ) and compared the percentage of NETs formed to PMA alone at $180 \mathrm{~min}$. Our results showed a significant reduction in PMA induced when cells were pre-treated with non-opsonized $F$. alocis compared to PMA alone (Fig. 4-9 A-B).

Now that we have determined what occurs with $F$. alocis pre-treatment of neutrophils before challenge with PMA, a pharmacological inducer of NETs, we sought to determine if $F$. alocis has the capabilities of manipulating the NET-forming neutrophils prior to exposure to known oral bacterial-inducers $S$. gordonii and $P$. stomatis. We pre-treated neutrophils with nonopsonized F. alocis for 30-60 min before $S$. gordonii challenge (180 min) and compared the percentage of NETs formed to $S$. gordonii alone at $180 \mathrm{~min}$. There was no significant change in NET induction by $S$. gordonii when cells were pre-treated with non-opsonized $F$. alocis for 30 min or 60 min compared to $S$. gordonii alone (Fig. 4-10 A). Additionally, we pre-treated neutrophils with opsonized $F$. alocis for 30-60 min before $S$. gordonii challenge (180 min) and 
compared the percentage of NETs formed to $S$. gordonii alone at 180 min. However, unlike with non-opsonized bacteria, there was a significant decrease in NET induction by $S$. gordonii when cells were pre-treated with opsonized $F$. alocis for $30 \mathrm{~min}$ and $60 \mathrm{~min}$ compared to S. gordonii alone (Fig. 4-10 B). Next, we pre-treated neutrophils with non-opsonized F. alocis for 30-60 min before $P$. stomatis challenge $(180 \mathrm{~min})$ and compared the percentage of NETs formed to $P$. stomatis alone at $180 \mathrm{~min}$. There was no significant change in NET induction between nonopsonized or opsonized $F$. alocis pre-treatment at $30 \mathrm{~min}$ or $60 \mathrm{~min}$ time point before $P$. stomatis challenge compared to P. stomatis alone (Fig. 4-10 C, D). These results indicate that $F$. alocis requires pre-treatment time prior to presence of positive NET stimulator to inhibit them, which means that the bacterium manipulates the neutrophils so that NET formation will not be triggered. Due to the results we obtained in Figures 4-8-11, it is more likely that F. alocis is manipulating neutrophil signaling mechanisms as opposed to DNase, nuclease or thermonuclease secretion, unless the secretion of the enzymes is too low in concentration to have an impact. $\underline{\text { S. gordonii and P. stomatis induce NETs in an ROS-dependent manner. }}$

The rapid formation of NETs seems to use an oxidase-independent process that does not involve cell death, where the lengthy NET induction is often oxidant-dependent and is considered a form of cell death $(155,366)$. It has been determined that $S$. gordonii and $P$. stomatis induce significant NET formation, therefore we sought to determine if these bacteria induced NETs in an ROS-dependent or independent manner. To do so, we exposed neutrophils to diphenyleneiodonium (DPI), a known inhibitor of NADPH oxidase activity, prior to challenge with $S$. gordonii or $P$. stomatis. As a control, we pre-treated neutrophils with DPI and then challenged with PMA, a known ROS-dependent inducer of NETs, to ensure our DPI was working effectively to inhibit the oxidase (Fig. 4-11 A, B). To accomplish this, we performed immunofluorescence microscopy where we exposed neutrophils to DPI and then challenged neutrophils with S. gordonii (MOI 1050-100) for 180 min and compared the NET induction to S. gordonii alone (MOI 10-50-100) for 180 min. It was observed that at MOI 50 and MOI 100, DPI pre-treatment significantly reduced 
NET formation compared to $S$. gordonii alone indicating that $S$. gordonii may induce NETs in an ROS and MOI-dependent manner (Fig. 4-11 A).

Similarly, we performed this assay with exposure of neutrophils to DPI and then challenged neutrophils with $P$. stomatis (MOI 10-50-100) for $180 \mathrm{~min}$ and compared the NET induction to $P$. stomatis alone for $180 \mathrm{~min}$ (Fig. 4-11 B). It was observed that only at MOI 100, DPI pre-treatment significantly reduced NET formation compared to $P$. stomatis alone indicating that $P$. stomatis may induce NETs in an ROS-dependent manner (Fig. 4-11 B).

\section{Discussion}

In vivo observation of neutrophils revealed their presence in the dental plaque and that NET formation is detected in the oral biofilm, the saliva, and also the crevicular exudate (155, 197, 380-382). Due to the large quantity of bacteria present dispersed throughout the gingival crevice, phagocytosis is an ineffective means for bacterial control, therefore NETs could serve as a more effective strategy for neutrophils to respond to infection (197). Furthermore, when these dispersed bacteria attempt to adhere to the gingival epithelium, they encounter NETs, which impair their chances for attachment and colonization $(197,367,382)$.

Given the importance of neutrophils to the field of periodontology, being the principal inflammatory cell, NET formation needs further study to determine its potential to periodontal disease pathogenesis $(363,373)$. Little has been characterized in the periodontal field in regard to NET formation; however, it has been implicated to occur as it is involved in other chronic inflammatory conditions. Due to the presence of a functional peptidyl arginine deiminase (PAD) enzyme in $P$. gingivalis, it is speculated that the bacterium can citrullinate its own proteins as well as host proteins, which is important for inducing NET release (363). In addition to chronic inflammatory diseases, NETs have been suggested to have a role in cancer and metastasis (166, $383)$. 
NET components found decorated within the extracellular matrix each play important roles in the stages of neutrophil activation and eventual release from neutrophils. First, neutrophil elastase (NE), a neutrophil-specific serine protease, functions in histone degradation, promotion of chromatin decondensation in the neutrophil nucleus, degradation of the nuclear envelope, antimicrobial activities within the neutrophil phagosome $(170,376,377)$. Studies performed with both NE and MPO knockout mice show failed induction of NETs and an increased susceptibility to infection (170). Tightly associated with NE is myeloperoxidase, which is necessary for NE, as it functions in conjunction with the ROS to translocate NE to the neutrophil nucleus (376). MPO is an enzyme that works to consume hydrogen peroxide in order to form hypochlorous acid (HOCl) among other important anti-microbial oxidants (376). There are four core histones in NETs (H2A, H2B, H3 and H4), which are responsible for the majority of their protein mass and serve as potent antimicrobials (168). Another important enzyme for NET production is peptidyl arginine deiminase-4, which functions in decondensation of the nuclear chromatin through citrullination of histones and also degradation of the nuclear envelope $(149,377)$. Similarly, as observed with knock-out studies in NE and MPO, PAD4 knock out mice are impaired in their NET capabilities $(377,384)$.

Neutrophil elastase is an enzyme that is believed to be crucial for the successful formation of NETs. We detected low levels of NE release by both non-opsonized and opsonized F. alocis across a time course, which indicates that this component could help to explain why NETs are not induced by this oral bacterium.

NET formation, depending on the stimuli, can occur rapidly, as observed with 10 min of Staphylococcus aureus challenge (363) or take more time to occur, as observed with 90-180 min of PMA treatment $(157,364,385)$. The rapid formation of NETs seems to use an oxidaseindependent process that does not involve cell death, where the lengthy NET induction is often oxidant-dependent and is considered a form of cell death $(155,366)$. As it has been determined that NETs can be induced at an early or late time point depending on the stimulus, time course 
studies with $F$. alocis allowed us to determine if induction of NETs was time-dependent and potentially whether it operated through an ROS-dependent or ROS-independent mechanism. Our observations showed that there is no significant induction of NETs at any time point.

It is known that different factors can impact and influence the capability of NET production, one of these factors being the multiplicity of infection (MOI) of the bacterial challenge (168). Hirschfeld et al reports that Aggregatibacter actinomycetemcomitans will induce NETs, but only at the high level of MOI 100 (378). It has been reported that the gingipain mutant strains K1A (Kgp) and E8 (RgpA/B) of P. gingivalis can induce NETs, however the wild type ATCC33277 and W50 strains do not induce them (306). Studies performed with Burkholderia psuedomallei showed that with increasing MOI and later time intervals, more NETs were formed (379). It is typical that with an increase in bacterial MOI, a minimal or intermediary NET induction may progress to a higher percentage of NETs formed. However, in our work, increasing the MOI of F. alocis did not impact the formation of NETs, which suggests that MOI increase does not promote enhanced NET formation.

In Scharrig et al, live Leptospira spp. were observed to induce significantly more NETs compared to heat-inactivated Leptospira spp. (386). In assays performed with B. pseudomallei, it was determined that killed bacteria induced significantly more NETs than live bacteria (379). However, when challenging neutrophils with heat-killed $F$. alocis, the bacterium did not induce NET formation. This suggests that $F$. alocis must not require viability to perform its manipulation of neutrophils, as it may employ heat-stable effector mechanisms.

It has been suggested that NET formation can impact the colonization of oral bacteria, which may impact the ability of $S$. gordonii to attach to the surface of the tooth and further effect other bacteria from residing on the early colonizers, disrupting the entire biofilm architecture. Unlike $F$. alocis, we observed both $S$. gordonii and $P$. stomatis induced NETs in an MOIdependent manner. Hirschfield et al reported NET formation was observed by a variety of oral bacteria, including $S$. gordonii, taken from supragingival biofilm and whole saliva samples in 
healthy donors (380). Similarly to the observations obtained with my data, $S$. gordonii challenge at MOI 10 produced $\sim 10 \%$ NET formation (380). These results suggest that $F$. alocis may represent a unique species to the oral community, as NET formation is not observed.

Although previous work demonstrates that "primed" or pre-activated neutrophils can induce NET formation, when priming neutrophils with TNF-alpha (72), it does not seem to preactivate neutrophils to form NETs in response to secondary bacterial stimulation of $F$. alocis. These results show that priming of neutrophils is not successful in promoting the NET formation by $F$. alocis. Future studies could be used to determine if these results are specific to TNF priming or if this result would change with a different priming agent.

Depending on the stimuli used to activate neutrophils, activation of the NADPH oxidase and production of intracellular ROS may or may not be required for NET formation $(157,167$, 364-366). Additionally, PMA induction of NETs usually occurs after 90 min of stimulation, but the ROS response happens within minutes, therefore a certain threshold level of ROS may be required for trigger of NETs (387). It has been reported that NETs may function to trap and kill microbes, or they may only be able to trap and the microbe evades the killing mechanism (149, $151,386,388,389)$. In the context of Streptococcus pneumoniae, NETs are effective in trapping bacteria, reducing the spread of further infection, however ineffective at killing the microbe (151). Studies performed by Wang et al determined that even different strains of the same species, as shown with Klebsiella pneumoniae, can be more resistant to trapping or killing by NETs (390). However, capturing the bacteria in its trap still provides benefit to the neutrophil, as it can limit dissemination of the bacteria $(149,151,386,388,389)$.

While the potential signaling pathways leading to NET formation have been outlined, few studies have been able to directly tie signaling mechanism inhibition with NET inhibition. A recent study found that TLR signaling could be linked to NET formation, as observed with antiinflammatory drug, dexamethasone (DXM) treatment on S. aureus and PMA-induced NETs (391). In S. aureus-induced NET formation it was concluded that TLR2 and TLR4 are involved, 
as DXM treatment inhibited NET formation, which could be rescued using TLR2 and TLR4 agonists (391).

The first link was established between NET degradation and bacterial pathogenicity (Group A streptococcus) as inhibition of GAS DNase led to enhanced pathogen clearance by neutrophils in vitro and less virulence in vivo (368). It has been reported that numerous Gram positive bacteria, like $S$. pneumoniae, will express DNases, which can aid in NET degradation (151). The production of extracellular nucleases is well known for bacterial pathogens, and were first reported to be active in anaerobic bacteria in 1974, but their role in virulence was only recently appreciated $(148,367)$. Pathogens can employ nucleases to aid in their resistance to NET-mediated killing mechanisms $(171,392)$. Presence of extracellular nucleases has been reported in these Gram-positive pathogens: Staphylococcus aureus, Streptococcus pyogenes, Streptococcus agalactiae, Streptococcus suis, Streptococcus sanguinis, Streptococcus pneumoniae (151, 171, 367-369, 392-395). Additionally, Palmer et al reported that periodontopathogenic bacteria can produce extracellular nucleases $(367,375)$. Recently, it was determined that Neisseria gonorrhoeae encodes for a heat-stable thermonuclease (Nuc), which provides the bacterium an effective virulence factor against NETs, as it is capable of DNA degradation (396). After NETs were produced, the presence of thermonuclease aided the bacterium in degrading the DNA and enhancing their survival capabilities (396). No significant reduction in NET formation when NETs stimulated with PMA are then exposed to F. alocis broth or growth supernatant (data not shown) which suggests that although $F$. alocis possesses a thermonuclease, it may be present at a low concentration, therefore deeming it ineffective in degradation of NETs.

Another method employed by microbes to evade NET-mediated killing is molecular mimicry, where bacteria will mimic host-specific surfaces to evade detection and further inhibit initiation and activation of an immune response against them $(149,363)$. They can also conceal their antigenic molecules or alter their surface modifications or electrochemical charges, which 
all serve as virulence factors that aid in their stealthy escape from the host's detection, allowing them to persist and further infection and inflammation $(149,363,397)$.

Host-pathogen balance is a very important factor in maintaining homeostasis and health in the oral cavity and the disruption of this balance is what leads to "Polymicrobial Synergy and Dysbiosis" (PSD) (241). In a synergistic biofilm, oral bacteria operate in a biofilm community which leads to exacerbation of their virulence potential allowing them to survive against the host anti-bacterial mechanisms. Additionally, they cause significant host tissue damage, which allows them to thrive in a nutrient-rich environment where they can successfully colonize the gingival epithelium (241). Periodontal disease caused by polymicrobial synergy among periodontal bacteria in subgingival biofilms may be successful in their colonization of the region through their NET degradation capacities (367). It has been reported in a few studies that biofilm formation can inhibit NETs and this can be attributed to the extracellular matrix structure, as detached planktonic bacteria can induce NETs (398). NET formation is an effective mechanism employed by neutrophils to respond to infection, therefore, lack of NET formation has disease promoting implications. This phenomenon was observed with CGD patients; these patients do not have an operational (inactive) NADPH oxidase complex and suffer from recurrent infections $(168,387)$.

Given the importance of neutrophils to the field of periodontology, being the principal inflammatory cell, NET formation needs further study to determine its potential to periodontal disease pathogenesis $(363,373)$. Little has been characterized in the periodontal field in regard to NET formation; however, it has been implicated to occur as it is involved in other chronic inflammatory conditions.

As NET formation has been demonstrated to occur in the oral cavity, especially in the context of periodontal disease presence, we first wanted to determine if $F$. alocis is able to participate in or induce NET formation. We determined that $F$. alocis does not induce NET formation and that it may function to manipulate neutrophils by an unknown mechanism. This 
manipulation by $F$. alocis could serve to disrupt the biofilm community and impact their ability to colonize the host as the as well as manipulate the host in its ability to effectively detect and respond to microbes through NET formation.

NETs have potentially different kinetic and functional properties depending on where they are operating, whether it be in the bloodstream or in the tissue, at the site of an infection (384). If in the bloodstream, they could aid in spreading bacteria, however, if they are at the tissue-level, they may prevent adhesion and colonization of the host tissue by bacteria. There is also potential that NETs could also operate in a biofilm formation and overgrowth (380). This leads to the question of whether the presence of NETs may be of benefit to the host and limit disease progression or be detrimental to the host and further disease progression (155). It is also not well-defined if and how NETs are effectively being cleared from the host, which may propagate unnecessary host inflammation and damage (363). However, it is also a possibility that neutrophils or other immune cells in interaction with neutrophils have adapted to create inhibitory feedback mechanisms, sending signals to their neighboring cells not to produce any more NETs $(365,399)$.

A recent study has implicated the potential interplay between NETs and other neutrophil mechanisms, like phagocytosis and autophagy and it may be necessary to further define the kinetics of these neutrophil functional mechanisms (400). Many studies suggest that interplay may also occur between both signaling pathways and NETs components, which could be better defined to help distinguish why certain pathways/components are utilized $(157,365,401)$. It is controversial whether certain signaling pathways and NETs components are required, which needs to be better defined for many microbes. It is also a possibility that there are inhibitory feedback mechanisms, paracrine signaling between neutrophils sensing neighbors don't produce any more NETs. It is still to be determined what causes only a certain percentage of neutrophils to produce NETs and whether factors such as age and health status determine this ability $(365,402)$. 
Furthermore, it is important to determine if 'all NETs are created equal' (384) among pathogens and diseases and why they could be phenotypically and functionally similar or different. Through using patient studies, it will be possible to help determine what causes the inter-patient variability seen in NET formation and the impact this has on disease development and progression $(380,403)$. Studies have suggested that NETs could be useful as predictors of disease development $(170,404,405)$.

Numerous studies have implicated that future studies have the potential to develop NETs therapeutics. There is promising potential for development of molecules/drugs that will: inhibit nuclease activity, oxygen radicals or even certain signaling pathways, like the Raf-MEK-ERK $(171,387,406)$. It has also been suggested that DNase activity can be neutralized (368).

Although serum and complement have been observed to negatively impact NET formation as they can cause its degradation, when we challenged neutrophils with either nonopsonized or opsonized $F$. alocis, no NET formation was observed. This suggests serum opsonization does not impact our studies and that the presence of opsonins did not favor a phagocytic killing mechanism, such as NETs. It has been determined that the relationship between complement opsonization and NET formation is dependent on the bacterial stimulus, some examples including oral bacterial like A. actinomycetemcomitans and Fusobacterium nucleatum, impair NET formation when opsonized $(18,375,407)$, therefore this could explain the differences we observe in the manipulation of opsonized compared to non-opsonized F. alocis in relation to its manipulation capacities of NET formation when exposed to S. gordonii differentially than PMA. Additionally, S. gordonii may be impacted by the presence of opsonins on $F$. alocis, which may explain the decrease in NET formation when Op-F. alocis challenge precedes $S$. gordonii challenge.

Previous studies performed on pathogenic Bordatella parapertussia show its capable of inhibiting PMA-induced NET formation when pre-treated with the bacterium prior to exposure to PMA, through the use of adenylate cyclase toxin (CyaA), that operates by inhibition of ROS 
production (408). The only other bacteria known to be able to avoid NET induction, as determined using murine neutrophils and the HL-60 neutrophil-like cell line, is Lactobacillus rhamnosus, which is a probiotic (409). Similarly, with F. alocis pre-treatment prior to PMA exposure, we see an inhibition in NET formation, which may be due to an unknown virulence factor. This implicates the involvement of $F$. alocis in preventing excessive inflammation created by NET formation and further allowing evasion and potentially survival from neutrophils.

Based on survival studies with $F$. alocis and human neutrophils, we determined that although the bacterium is able to be effectively internalized, it can survive up to $4 \mathrm{hrs}$ intracellularly (See Chapter 3 Figure 3-1). Therefore, it is possible that F. alocis possesses a virulence factor that may facilitate its survival in phagocytic-based killing mechanisms, however, in the context of extracellular NET formation it is unclear whether $F$. alocis would survive. In our studies, we observe that $F$. alocis is effectively trapped in the NETs that are formed by other inducers, however further studies of bacterial killing are needed to determine if $F$. alocis is effectively killed in the NETs.

As it has been shown that the use of DPI inhibitor will target and inhibit ROS-dependent NET induction by impacting the NADPH oxidase complex, our studies with DPI inhibition showed that NET formation is ROS-dependent in both S. gordonii and P. stomatis.

Due to the lack of NET induction by F. alocis, we further looked into our proteomic analysis performed on the bacteria to determine if our bacteria possessed a DNase, nuclease or thermonuclease that may be aiding in the bacterium's ability to degrade NETs. We observed that F. alocis did possess an active and functional thermonuclease, however due to the low coverage and concentration of the enzyme in our culture, this may explain why, unlike observed with the thermonuclease in $N$. gonorrhoeae, NETs that are formed are not successfully degraded by $F$. alocis presence.

These results suggest that $F$. alocis is more likely to be manipulating the neutrophil NET signaling mechanisms, because if NETs are already formed, the bacterium is ineffective at 
degrading them; however, it prevents them from being formed even with exposure to a known stimulator of NET formation like PMA. Additionally, F. alocis is able to control the neutrophils at an earlier time point in their NET formation induced by $S$. gordonii than with PMA, which could be due to the potency and pharmacological nature of PMA. It is possible that $F$. alocis can inhibit a crucial pathway involved in the formation of NETs, for example, as the bacterium does not induce an ROS response and NET formation could be ROS-dependent, this could help to explain why $F$. alocis fails to induce NETs.

Disruption of the entire microbial community by NET formation could potentially inhibit colonization of primary colonizers, like $S$. gordonii, bacteria that are critical to the initial structure of the dental plaque. This will occur by competitive inhibition, whereby the NETs will colonize the space of the gingival epithelium, which will not allow for bacterial colonization $(367,382)$. As $S$. gordonii is also a commensal organism, it is possible that if it is not allowed to colonize the host due to NET formation, the host will be further disadvantaged when presented with a pathogenic organism. However, much to the detriment of the host, NETs can also serve as a potential substrate for bacterial attachment in building their biofilm.

F. alocis is able to evade detection and a potential killing by neutrophils via NETs, as it does not induce NETs and could inhibit their formation. However, it is possible that the entire biofilm community benefits from $F$. alocis as its presence is known to manipulate neutrophils, leading to the potential for other bacteria that would normally be recognized and effectively killed by NETs go undetected, survive and persist in the host. This could be especially important for those bacteria that are not effectively internalized and neutrophils rely on extracellular killing mechanisms to effectively clear the pathogen.

Material and Methods

Neutrophil isolation. 
Neutrophils were isolated from blood of healthy donors using plasma-Percoll gradients as previously described (146), and in accordance with the guidelines approved by the Institutional Review Board of the University of Louisville. Microscopic evaluation of the isolated cells showed that $>95 \%$ of the cells were neutrophils. Trypan blue exclusion indicated that $>97 \%$ of cells were viable.

Bacterial strains, growth conditions and preparation.

F. alocis ATCC 38596 was cultured in brain heart infusion (BHI) broth supplemented with L-cysteine $(0.1 \%)$ and arginine $(20 \%)$ for 7 days anaerobically at $37^{\circ} \mathrm{C}$ as previously described $[27$, 42]. Opsonized F. alocis was prepared in $10 \%$ normal human serum at $37^{\circ} \mathrm{C}$ for $20 \mathrm{~min}$ and cultures were washed three times with PBS prior to use (Complement Technology, Tyler, Texas). For viability studies, heat killed $F$. alocis was generated by incubation at $90{ }^{\circ} \mathrm{C}$ for $60 \mathrm{~min}$. For fluorescence microscopy assays, F. alocis was labeled with 5(6)-Carboxyfluorescein $N$ hydroxysuccinimide ester (CFSE; Life Technologies, $4 \mathrm{mg} / \mathrm{mL}$ ) for $30 \mathrm{~min}$ at room temperature in the dark, and the cultures were washed three times with PBS prior to use.

S. gordonii strain DL1 was cultured in BHI broth overnight anaerobically at $37^{\circ} \mathrm{C} . S$. gordonii was labeled with CFSE (4 mg/mL) or Hexidium iodide (HI; Life Technologies, $5 \mathrm{mg} / \mathrm{mL}$ ) for $30 \mathrm{~min}$ at room temperature in the dark, and the cultures were washed three times with PBS prior to use.

P. stomatis strain CM2 was cultured in tryptic soy broth supplemented with $20 \mathrm{~g} /$ liter yeast extract, $1 \%$ hemin, and $1 \%$ reducing agent $\left(37.5 \mathrm{~g} /\right.$ liter $\mathrm{NH}_{4} \mathrm{Cl}, 25 \mathrm{~g} /$ liter $\mathrm{MgCl}_{2} \cdot 6 \mathrm{H}_{2} \mathrm{O}, 5 \mathrm{~g} / \mathrm{liter}$ $\mathrm{CaCl}_{2} \cdot 2 \mathrm{H}_{2} \mathrm{O}, 50 \mathrm{~g} /$ liter L-cysteine $\mathrm{HCl}, 5 \mathrm{~g} /$ liter $\mathrm{FeCl}_{2} \cdot 4 \mathrm{H}_{2} \mathrm{O}$ ) overnight anaerobically at $37^{\circ} \mathrm{C}$. $P$. stomatis was labeled with CFSE (4 mg/mL) or Hexidium iodide (HI; Life Technologies, $5 \mathrm{mg} / \mathrm{mL}$ ) for $30 \mathrm{~min}$ at room temperature in the dark, and the cultures were washed three times with PBS prior to use.

NETs immunofluorescence microscopy. 
To assess NET formation of neutrophils, we used an adaption of a previously described method (410). Neutrophils ( 1 x $10^{6}$ cells/condition) were seeded onto sterile $12 \mathrm{~mm}$ coverslips in a 24-well plate in NETs assay media (RPMI + 0.5\% BSA + $10 \mathrm{mM}$ HEPES) and incubated for 1 $\mathrm{h}$ in a tissue culture incubator at $37^{\circ} \mathrm{C}$ to allow cells to attach to coverslips. After $1 \mathrm{~h}$ incubation, neutrophils were left unstimulated, or stimulated with Phorbol 12-myristate 13-acetate (PMA, Sigma, $50 \mathrm{nM}, 180 \mathrm{~min}$ ), or challenged with CFSE-labeled non-opsonized $F$. alocis (MOI 10-50100, 15-30-60-90-180 min), or with CFSE-labeled opsonized F. alocis (MOI 10-50-100, 15-3060-90-180 min), or with CFSE-labeled non-opsonized heat-killed F. alocis (MOI 10-50-100, 1530-60-90-180 min), or with CFSE-labeled opsonized heat-killed F. alocis (MOI 10-50-100, 1530-60-90-180 min), or with CFSE-labeled S. gordonii (MOI 10-50-100, $180 \mathrm{~min}$ ), or with HIlabeled S. gordonii (MOI 10-50-100, 90-180 min), or with CFSE-labeled P. stomatis (MOI 1050-100, $180 \mathrm{~min}$ ), or with HI-labeled P. stomatis (MOI 10-50-100, 90-180 min).

For co-infection studies, neutrophils were challenged with HI-labeled S. gordonii (MOI 100) + CFSE-labeled non-opsonized $F$. alocis (MOI 10) or CFSE-labeled opsonized F. alocis (MOI 10) for 180 min, or with HI-labeled P. stomatis (MOI 50) + CFSE-labeled non-opsonized F. alocis (MOI 10) or CFSE-labeled opsonized F. alocis (MOI 10) for $180 \mathrm{~min}$.

For pre-treatment studies, neutrophils were challenged with HI-labeled S. gordonii (MOI 100) for $90 \mathrm{~min}+$ CFSE-labeled non-opsonized F. alocis (MOI 10) or CFSE-labeled opsonized F. alocis (MOI 10) for $90 \mathrm{~min}$, or with HI-labeled P. stomatis (MOI 50) for $90 \mathrm{~min}+$ CFSElabeled non-opsonized F. alocis (MOI 10) or CFSE-labeled opsonized F. alocis (MOI 10) for 90 min, or stimulated with TNF- $\alpha(10 \mathrm{~min}, 2 \mathrm{mg} / \mathrm{mL})$, or stimulated with TNF- $\alpha(10 \mathrm{~min})$ and then challenged with CFSE-labeled non-opsonized $F$. alocis (MOI 10, $180 \mathrm{~min}$ ), or with CFSE-labeled opsonized $F$. alocis (MOI 10, $180 \mathrm{~min}$ ) or challenged with CFSE-labeled non-opsonized $F$. alocis (MOI 10, 30-60 min) or with CFSE-labeled opsonized F. alocis (MOI 10, 30-60 min) and then stimulated with PMA (180 min) or challenged with HI-labeled S. gordonii (MOI 100, $180 \mathrm{~min}$ ) or challenged with HI-labeled $P$. stomatis (MOI 50). 
For inhibition studies, neutrophils were stimulated with diphenyleneiodonium chloride, (DPI, Sigma, $10 \mu \mathrm{M}$ ), an inhibitor of the NAPDH oxidase, and then stimulated with PMA for 180 min, or challenged with HI-labeled S. gordonii (MOI 10-50-100, $180 \mathrm{~min}$ ), or challenged with HI-labeled P. stomatis (MOI 10-50-100, $180 \mathrm{~min}$ ).

Following challenge of neutrophils, for bacterial-challenged conditions, phagocytosis was synchronized by centrifugation at $600 \mathrm{x} g$ at $14^{\circ} \mathrm{C}$ and plates were put in a $37^{\circ} \mathrm{C}$ cell culture incubator for time point (15-30-60-90-180 min). After challenge, cells were fixed in 2\% paraformaldehyde (PFA) for $2 \mathrm{~h}$, washed in PBS 3 times for 5 min and then blocked overnight at $4^{\circ} \mathrm{C}$ with $1 \%$ BSA. After overnight blocking, cells were stained with MPO antibody (Biolegend, $667802,1: 1000$ ) in a $37^{\circ} \mathrm{C}$ cell culture incubator for $1 \mathrm{~h}$. After wash in PBS 3 times for $5 \mathrm{~min}$, cells were stained with secondary antibody AlexaFluor 647 (Life Technologies, 1:1000) in a $37^{\circ} \mathrm{C}$ cell culture incubator for $1 \mathrm{~h}$ and washed in PBS 3 times for $5 \mathrm{~min}$. DAPI $(3 \mu \mathrm{M})$ was applied for $5 \mathrm{~min}$ at room temperature as a nuclear stain and cells were washed in PBS 1 time for 5 min. Confocal images (1- $\mu \mathrm{m}$ thickness for each slice) were obtained using a Fluoview FV1000 confocal microscope with a $63 \mathrm{X}$ oil objective to determine NET induction. Ten images taken randomly from different regions of each coverslip in an experiment were taken.

To quantify the NET induction, we used methods previously described (374). The image files were loaded as separate image stacks for each channel in ImageJ/FIJI software. To collect the data of total cell number in the DAPI fluorescence image stack, automatic particle analysis was set to 20 pixels minimum size and summarized the result output. To collect the data of total cell number in the MPO fluorescence image stack, automatic particle analysis was set to 75 pixels minimum size and summarized the result output. The output list results were imported into an Excel spreadsheet for further processing. The percentage of NETs formed was calculated by the following formula:

NET-rate $(\%)=100 \times$ Objects counted (MPO channel) / Objects counted (DAPI channel). 
The percentage of NETs formed was calculated for each of the ten images per condition acquired and then summarized as an average per condition.

\section{Neutrophil elastase extracellular release assay.}

In order to detect neutrophil extracellular trap release, we utilized the Cayman Chemical NET assay kit, which allowed for the detection of extracellular neutrophil elastase (NE). The assay was performed according to the manufacturer's protocol. Neutrophils $\left(1 \times 10^{6}\right.$ cells/condition $)$ were seeded into a 24-well plate in NETs assay media (RPMI $+0.5 \% \mathrm{BSA}+1 \mathrm{M} \mathrm{CaCl}_{2}$ ) and incubated for $30 \mathrm{~min}$ in a tissue culture incubator at $37^{\circ} \mathrm{C}$ to allow cells to settle. After 30 min incubation, neutrophils $\left(1 \times 10^{6}\right.$ cells/condition) were left unstimulated, challenged with non-opsonized $F$. alocis, or with opsonized F. alocis at an MOI of 10 and phagocytosis was synchronized (for bacterial-challenged conditions) by centrifugation at $600 \mathrm{x} g$ at $14^{\circ} \mathrm{C}$. Plates were put in a tissue culture incubator at $37^{\circ} \mathrm{C}$ for $15-30-60-90 \mathrm{~min}$. Following challenge, conditions were aspirated and washed three times with NETs assay media to ensure removal of soluble neutrophil elastase (NE). Next, NET assay S7 nuclease was added to each condition and incubated for $15 \mathrm{~min}$ at $37^{\circ} \mathrm{C}$, to disrupt the NETs. The supernatants for each sample were then collected and NET assay EDTA solution was added to inactivate the nuclease. Cell supernatants were added to a 96-well plate. Next, NET assay neutrophil elastase substrate was added to each well. The plate was covered and incubated at $37^{\circ} \mathrm{C}$ for $2 \mathrm{~h}$. Following the $2 \mathrm{~h}$ incubation, the signal, detecting extracellular NE, was measured at $405 \mathrm{~nm}$ using a SpectraMax Soft Max Pro 5.4 spectrophotometer.

Statistical analysis.

For all the experimental conditions tested in this study, the statistical analysis used was a one-way ANOVA with the Tukey-Kramer multiple-comparison test (GraphPad Prism software, San Diego, CA, USA). Differences were considered statistically significant at the level of $\mathrm{P}<0.05$. 
$\underline{\text { Figures and Figure Legends }}$

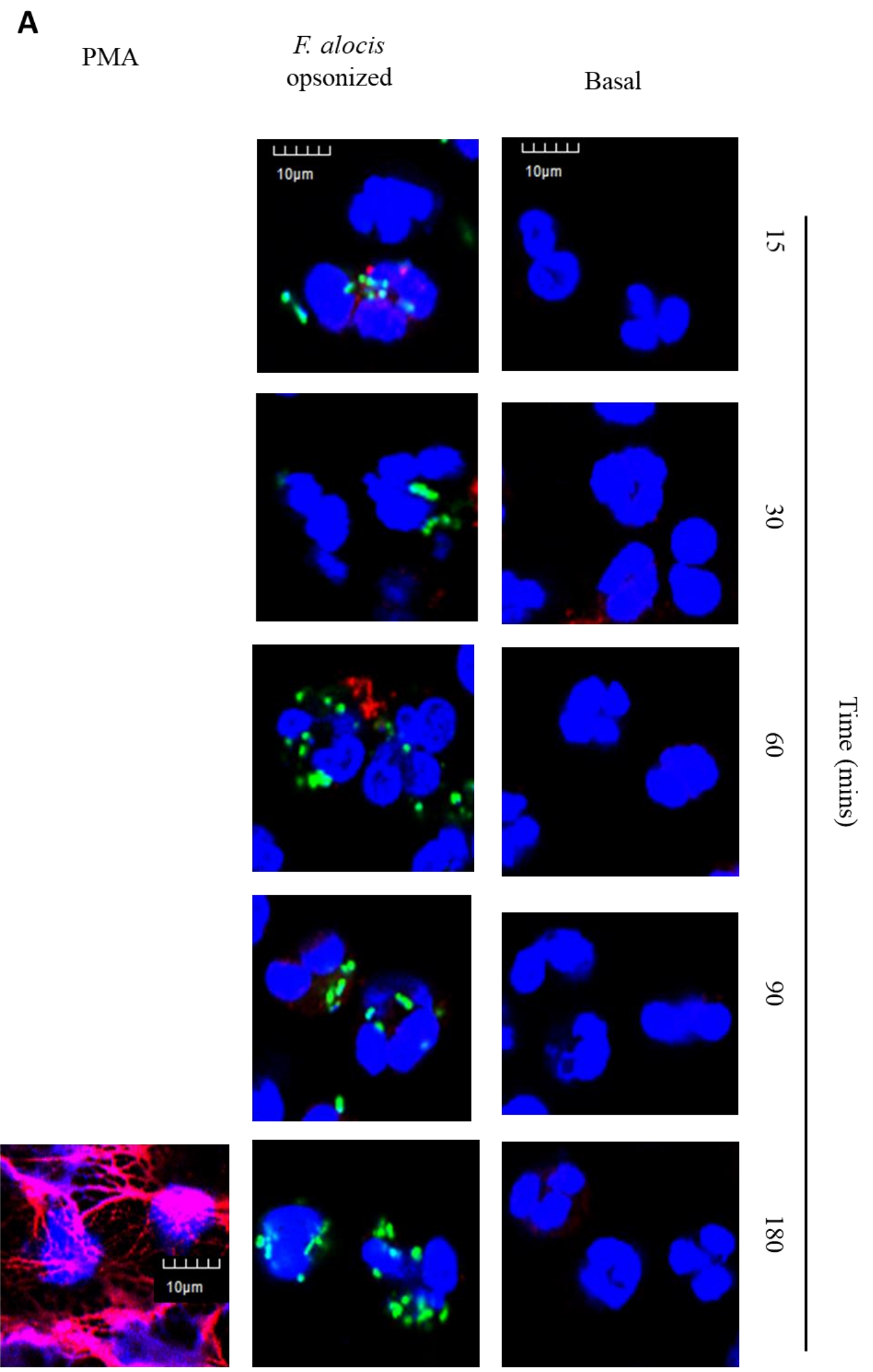




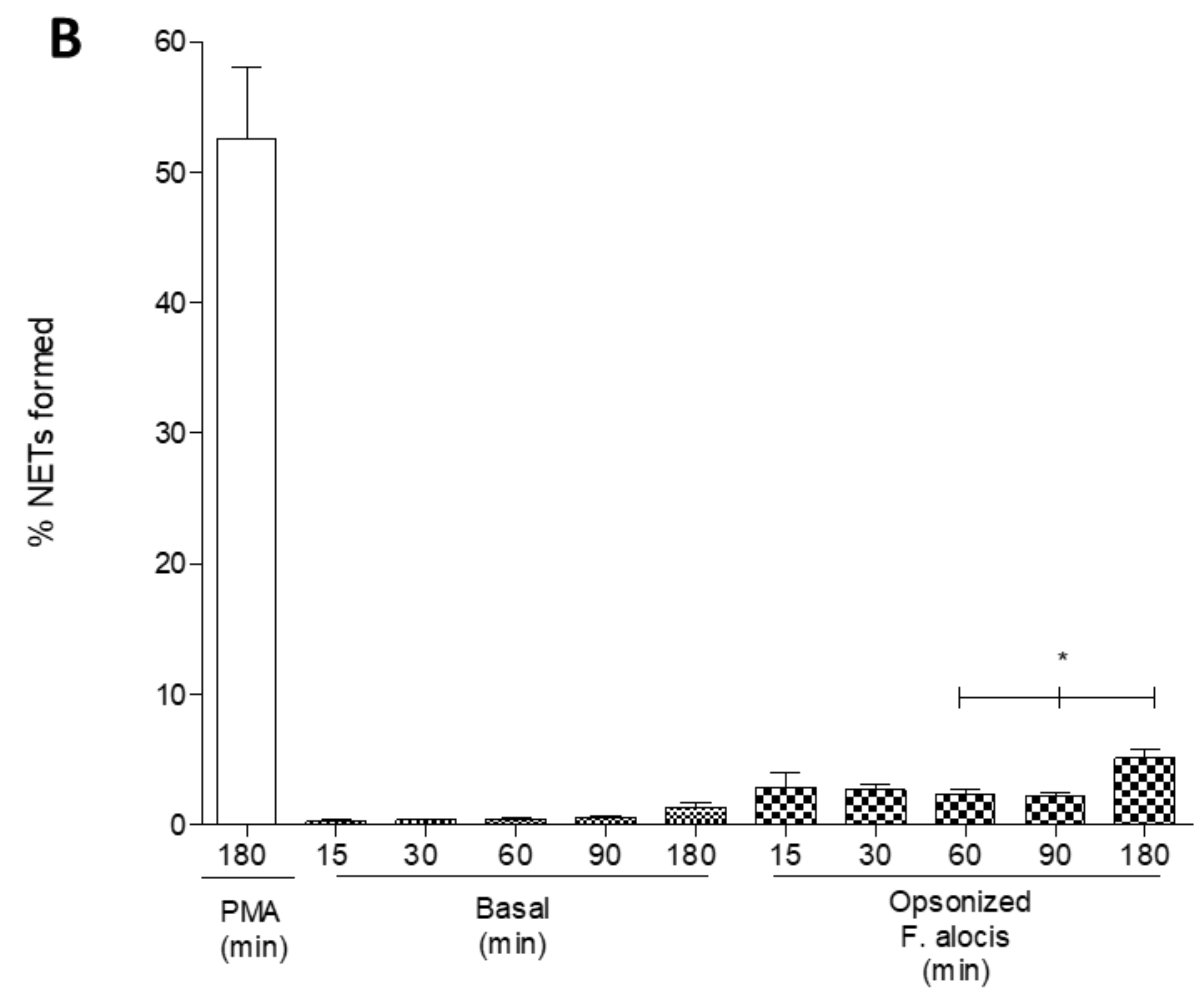

Figure 4-1. Opsonized $F$. alocis challenge fails to induce NET formation by human neutrophils.

Neutrophils were unchallenged (Basal), exposed to PMA-50 nM (180 min) or challenged with CFSE-labeled opsonized F. alocis (MOI 10) for 15-30-60-90-180 min. Following infection, cells were fixed, exposed to antibodies directed against MPO (Red- AlexaFluor647), stained with DAPI (blue), and then imaged for NET immunofluorescence by confocal microscopy. (A) Representative confocal images (from 3 replicates of 100 quantified cells) of unstimulated (Basal) neutrophils or neutrophils challenged with CFSE-labeled opsonized $F$. alocis-challenged neutrophils across a time course (15-30-60-90-180 min). CFSE (green): bacteria, DAPI (blue): neutrophil nucleus/DNA, AlexaFluor647 (red): MPO, Merge: NET formation. (B) Quantification, using ImageJ analysis (see details in Materials \& Methods), of percentage of NETs formed from unchallenged neutrophils (Basal), exposed to PMA (180 min) or challenged with opsonized $F$. alocis (15-30-60-90-180 min). Data are means $+/-$ SEM from 4 independent experiments. ${ }^{*} \mathrm{p}<0.05$. 
A PMA

$$
\begin{gathered}
\text { F. alocis } \\
\text { non-opsonized }
\end{gathered}
$$
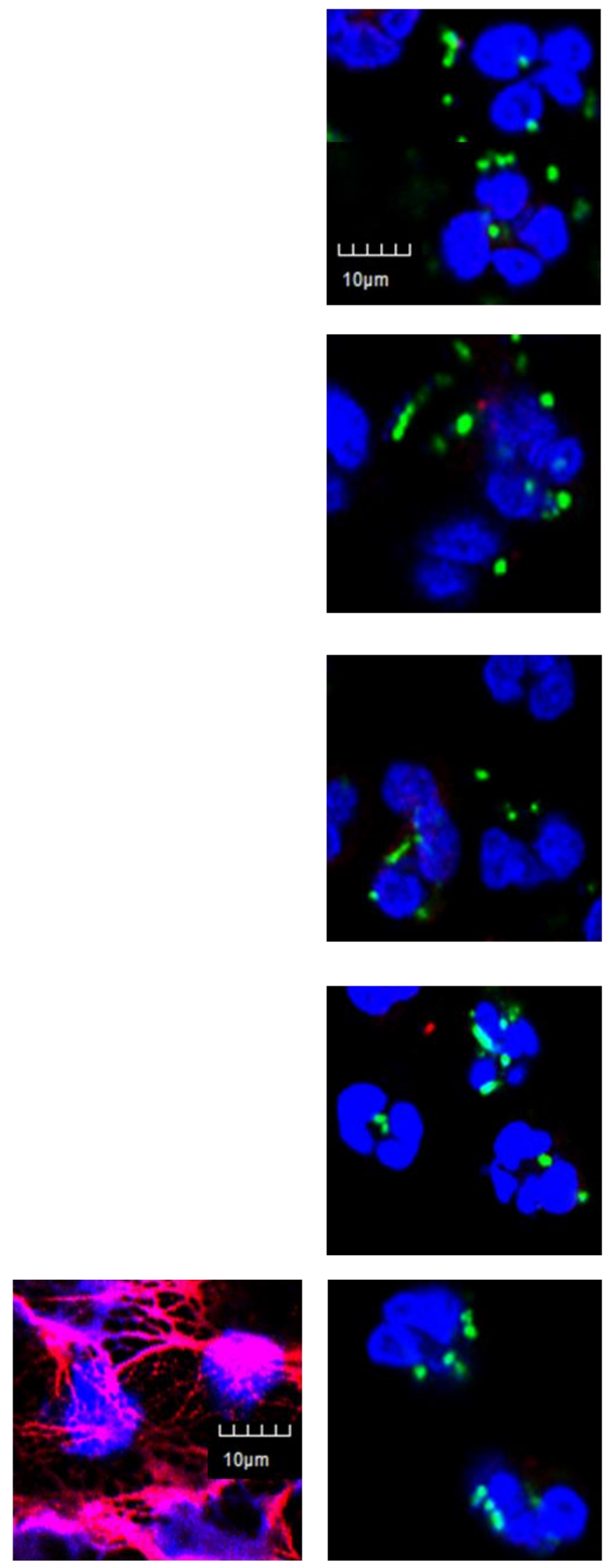

\section{Basal}

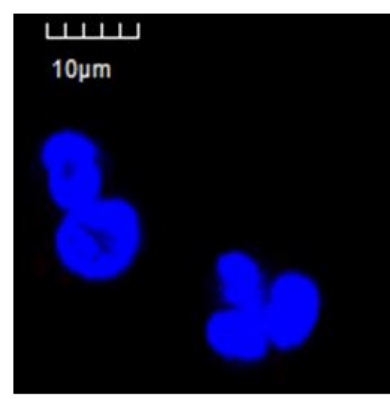

w

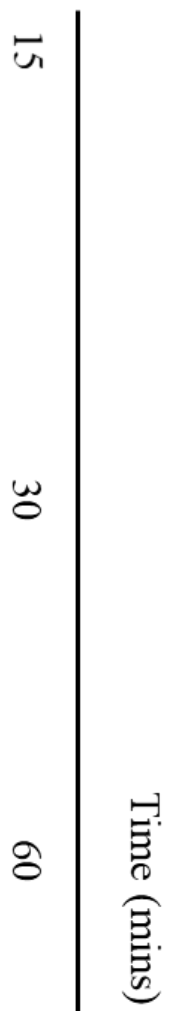

8

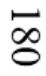
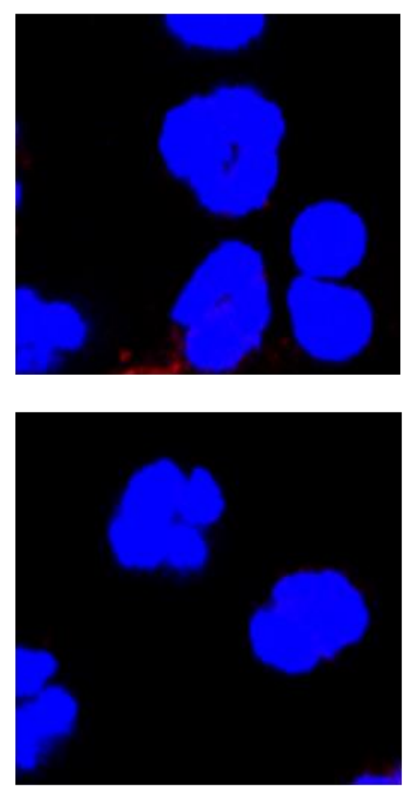

蔦
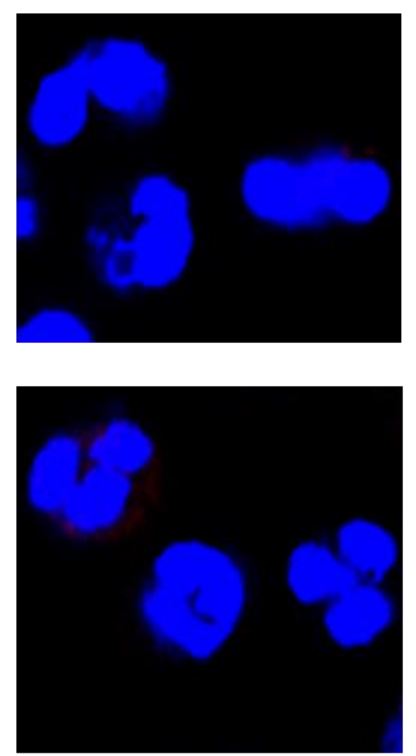
B

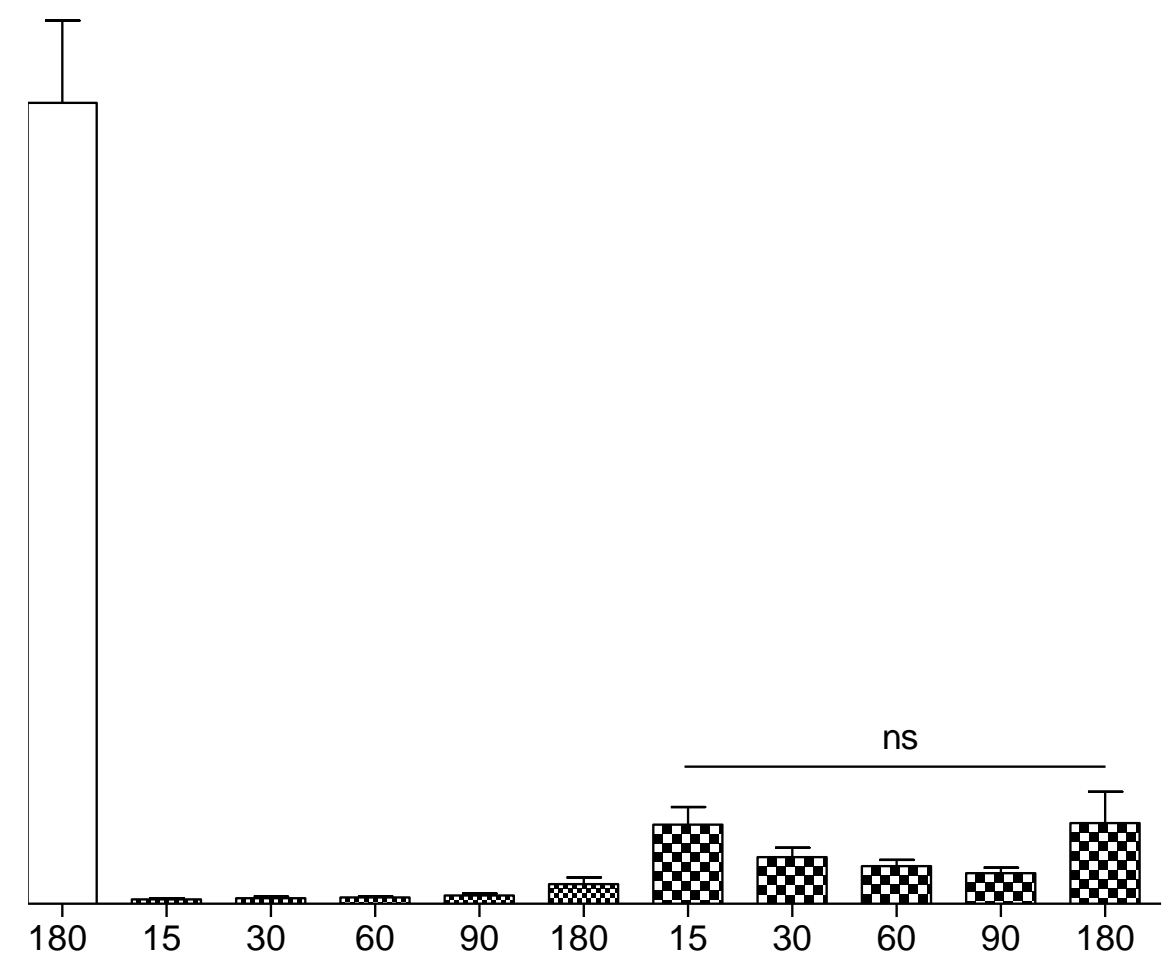

Figure 4-2. Non-opsonized $F$. alocis challenge fails to induce NET formation by human neutrophils.

Neutrophils were unchallenged (Basal), exposed to PMA-50 nM (180 min) or challenged with CFSE-labeled non-opsonized F. alocis (MOI 10) for 15-30-60-90-180 min. Following infection, cells were fixed, exposed to antibodies directed against MPO (Red- AlexaFluor647), stained with DAPI, and then imaged for NET immunofluorescence by confocal microscopy. (A) Representative confocal images (from 3 replicates of 100 quantified cells) of unstimulated (Basal) neutrophils or neutrophils challenged with CFSE-labeled opsonized F. alocis-challenged neutrophils across a time course (15-30-60-90-180 min). CFSE (green): bacteria, DAPI (blue): neutrophil nucleus/DNA, AlexaFluor647 (red): MPO, Merge: NET formation. (B) Representative confocal images (from 3 replicates of 100 quantified cells) of neutrophils challenged with CFSE- 
labeled non-opsonized $F$. alocis-challenged neutrophils across a time course (15-30-60-90-180 min). ns, nonsignificant. 

A F. alocis heat- $\quad$ F. alocis heat-killed killed opsonized non-opsonized
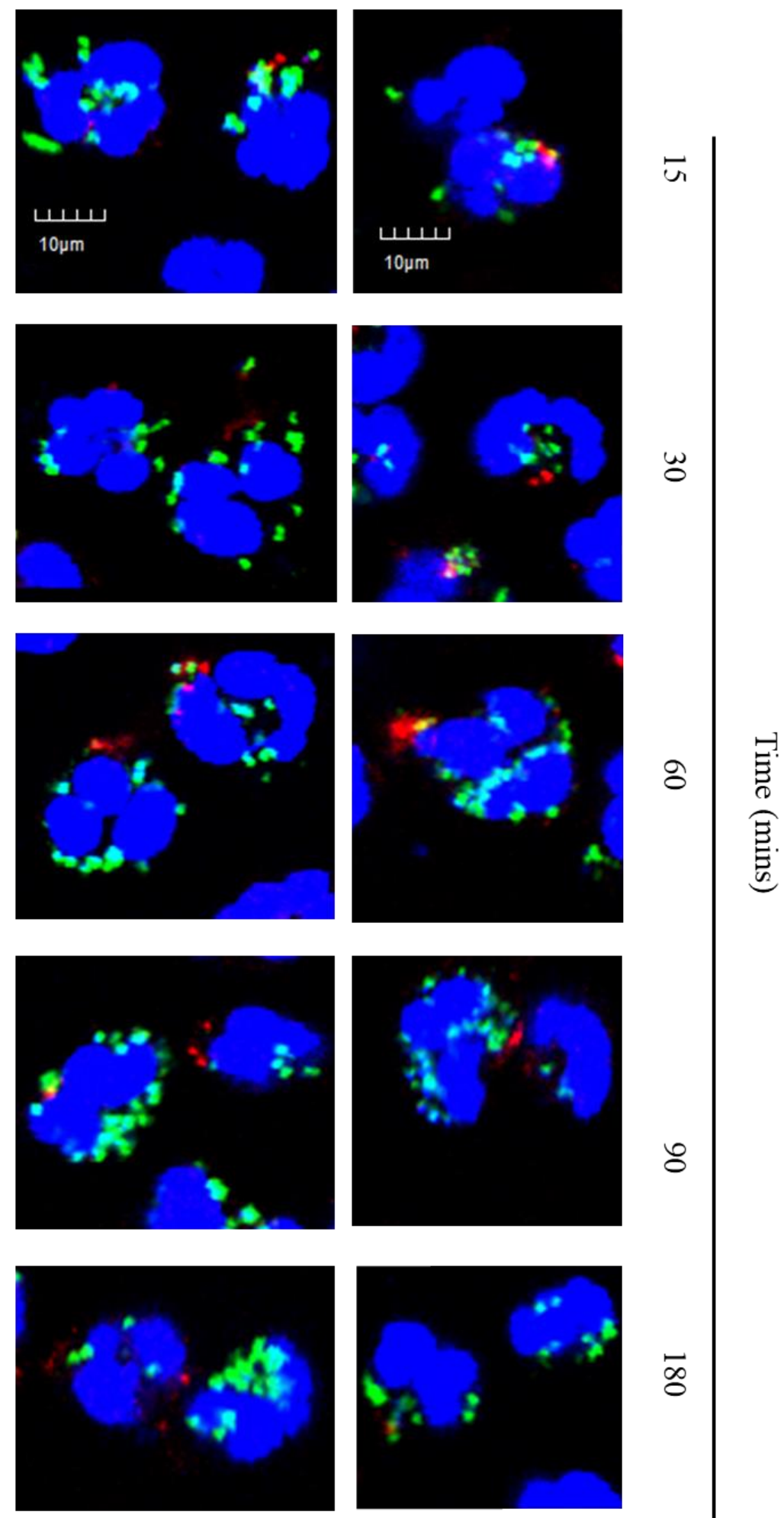

ळ 


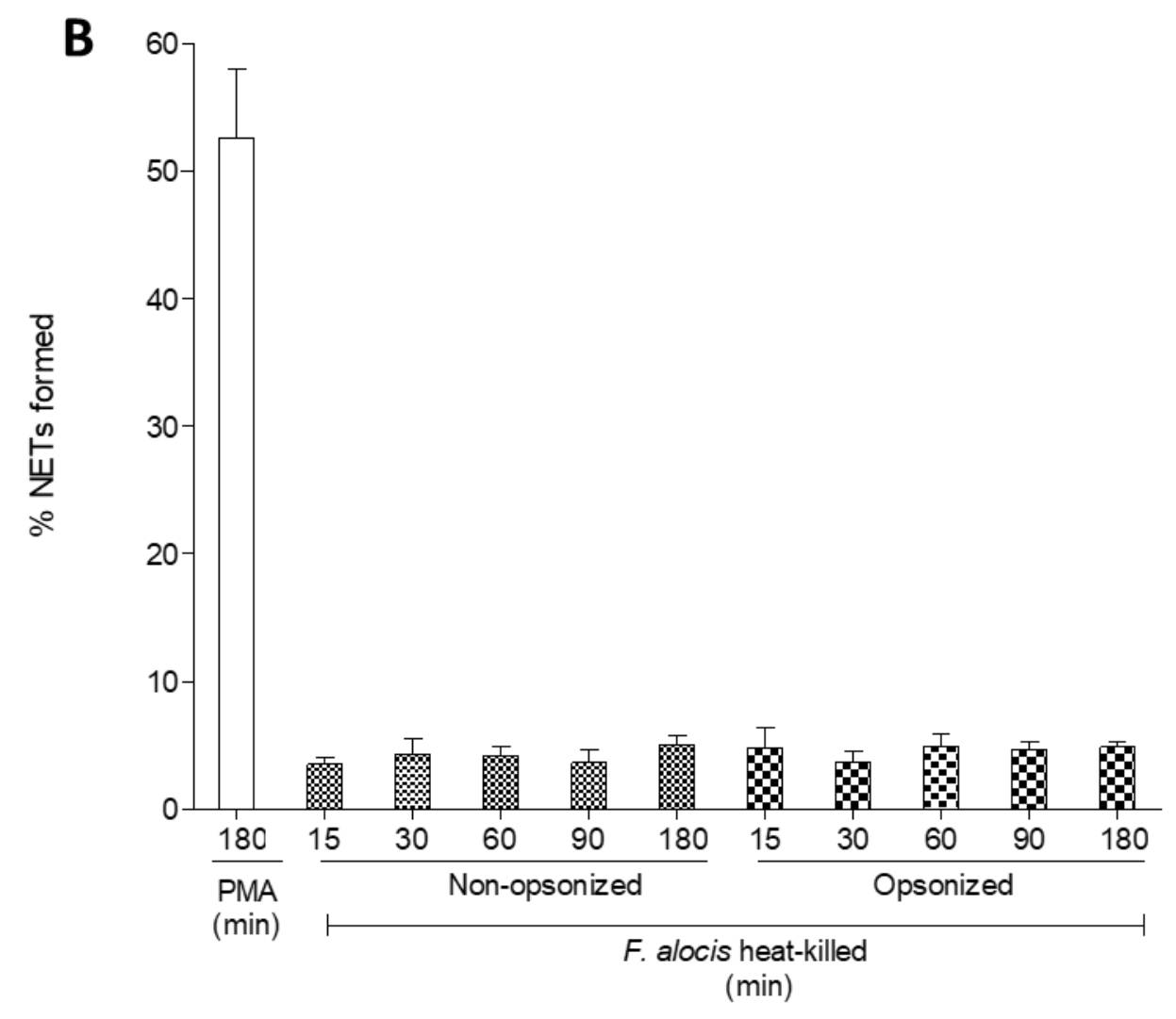

Figure 4-3. Heat-killed $F$. alocis challenge fails to induce NET formation by human neutrophils.

Neutrophils were unchallenged (Basal), exposed to PMA-50 nM (180 min) or challenged with heat-killed CFSE-labeled non-opsonized $F$. alocis (MOI 10), or CFSE-labeled heat-killed opsonized F. alocis (MOI 10) for 15-30-60-90-180 min. Following infection, cells were fixed, exposed to antibodies directed against MPO (Red- AlexaFluor647), stained with DAPI, and then imaged for NET immunofluorescence by confocal microscopy. (A) Representative confocal images (from 3 replicates of 100 quantified cells) of unstimulated (Basal) neutrophils or neutrophils challenged with heat-killed CFSE-labeled non-opsonized and heat-killed CFSElabeled opsonized F. alocis-challenged neutrophils across a time course (15-30-60-90-180 min). CFSE (green): bacteria, DAPI (blue): neutrophil nucleus/DNA, AlexaFluor647 (red): MPO, Merge: NET formation. (B) Quantification, using ImageJ analysis, of percentage of NETs formed 
from unchallenged neutrophils (Basal), exposed to PMA (180 min) or challenged with heat-killed non-opsonized F. alocis (15-30-60-90-180 min) or heat-killed opsonized F. alocis (15-30-60-90180 min). Data are means $+/-$ SEM from 3 independent experiments. 


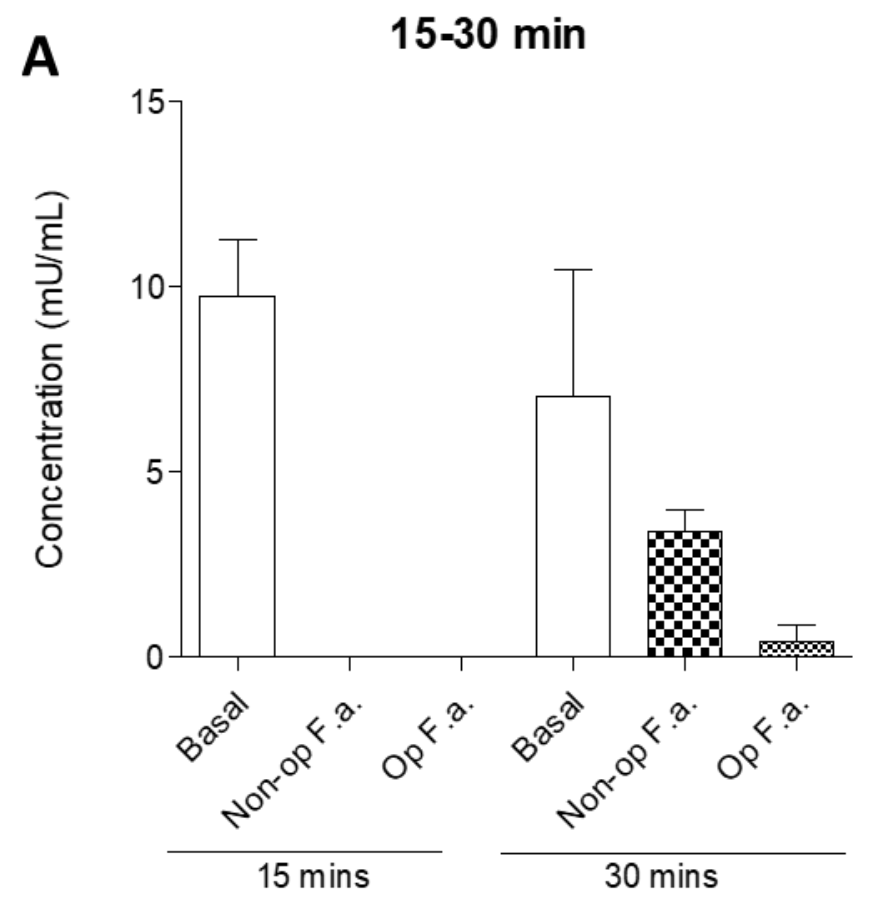

B

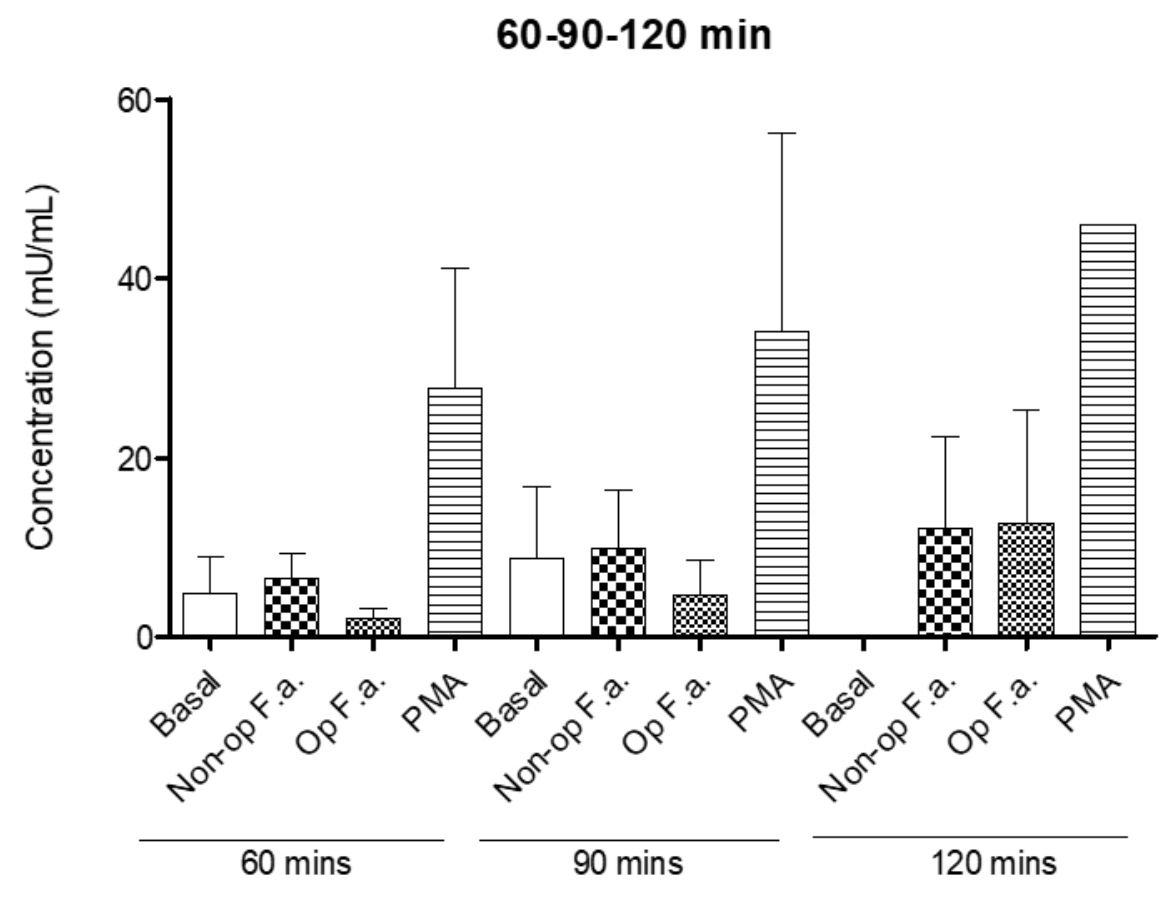


Figure 4-4. F. alocis fails to induce the release of extracellular neutrophil elastase.

(A) Neutrophils were left unstimulated (Basal), challenged with non-opsonized F. alocis (Non-op F.a.) or challenged with opsonized F. alocis (Op F.a.) for 15-30 min. (B) Neutrophils were left unstimulated (Basal), challenged with non-opsonized F. alocis (Non-op F.a.) or challenged with opsonized F. alocis (Op F.a.) for 60-90-120 min. As a positive control, neutrophils were stimulated with PMA (20 nM) for 60-90-120 min. Following treatment, soluble elastase was removed through aspiration and washing, and nuclease was added for $15 \mathrm{~min}$. Next, the supernatants were collected and EDTA solution was added. Supernatants were assessed for extracellular neutrophil elastase through the addition of elastase substrate to the samples. The samples were read by a spectrophotometer at $405 \mathrm{~nm}$. Data are means +/- SEM from 2 independent experiments. ${ }^{*} \mathrm{p}<0.05$ 


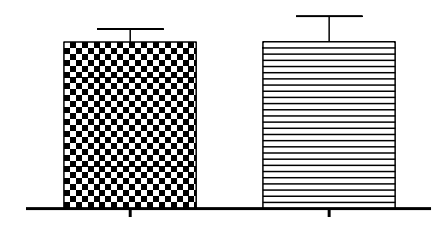

Figure 4-5. TNF- $\alpha$ pre-treatment fails to stimulate NET formation in response to challenge with $F$. alocis.

Neutrophils were stimulated with TNF- $\alpha$ (10 min) or challenged with CFSE-labeled nonopsonized F. alocis (MOI 10), CFSE-labeled opsonized F. alocis (MOI 10) for $180 \mathrm{~min}$, or pretreated with TNF- $\alpha$ (10 min) and then challenged with CFSE-labeled non-opsonized $F$. alocis (MOI 10), CFSE-labeled opsonized F. alocis (MOI 10) for 180 min. Following infection, cells were fixed, exposed to antibodies directed against MPO (Red- AlexaFluor647), stained with DAPI, and then imaged for NET immunofluorescence by confocal microscopy. Quantification, using ImageJ analysis, of percentage of NETs formed from neutrophils challenged with CFSElabeled non-opsonized $F$. alocis (MOI 10), CFSE-labeled opsonized F. alocis (MOI 10) for 180 min, or pre-treated with TNF- $\alpha(10 \mathrm{~min})$ and then challenged with CFSE-labeled non-opsonized F. alocis (MOI 10), CFSE-labeled opsonized F. alocis (MOI 10) for $180 \mathrm{~min}$. Data are means +/SEM from 4 independent experiments. 

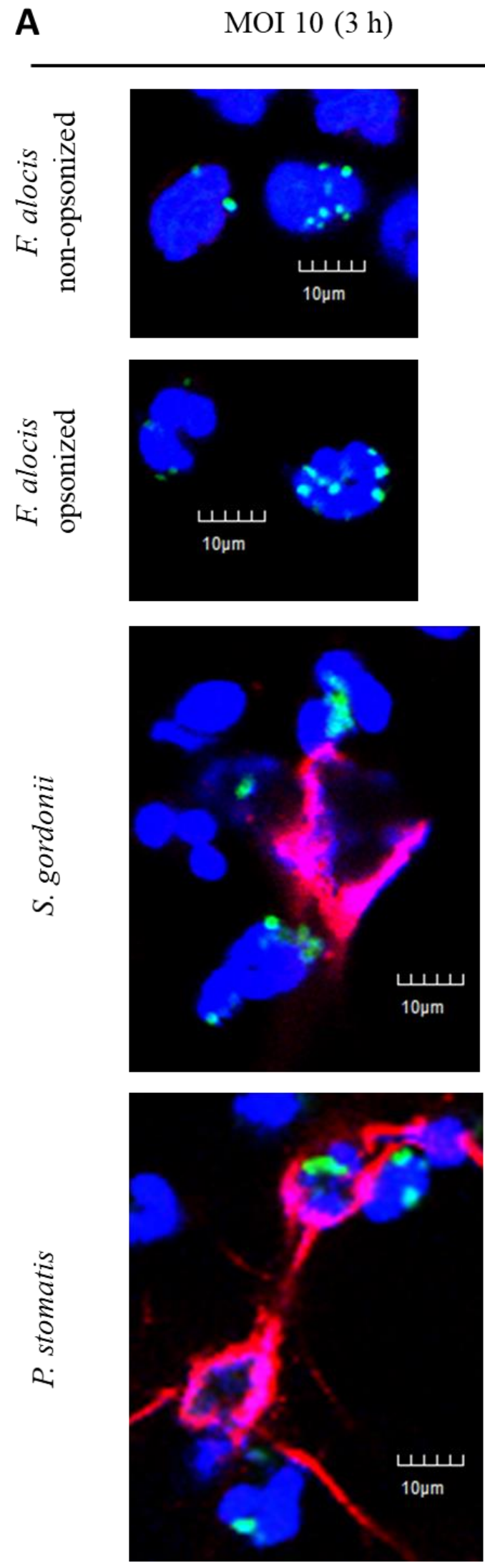


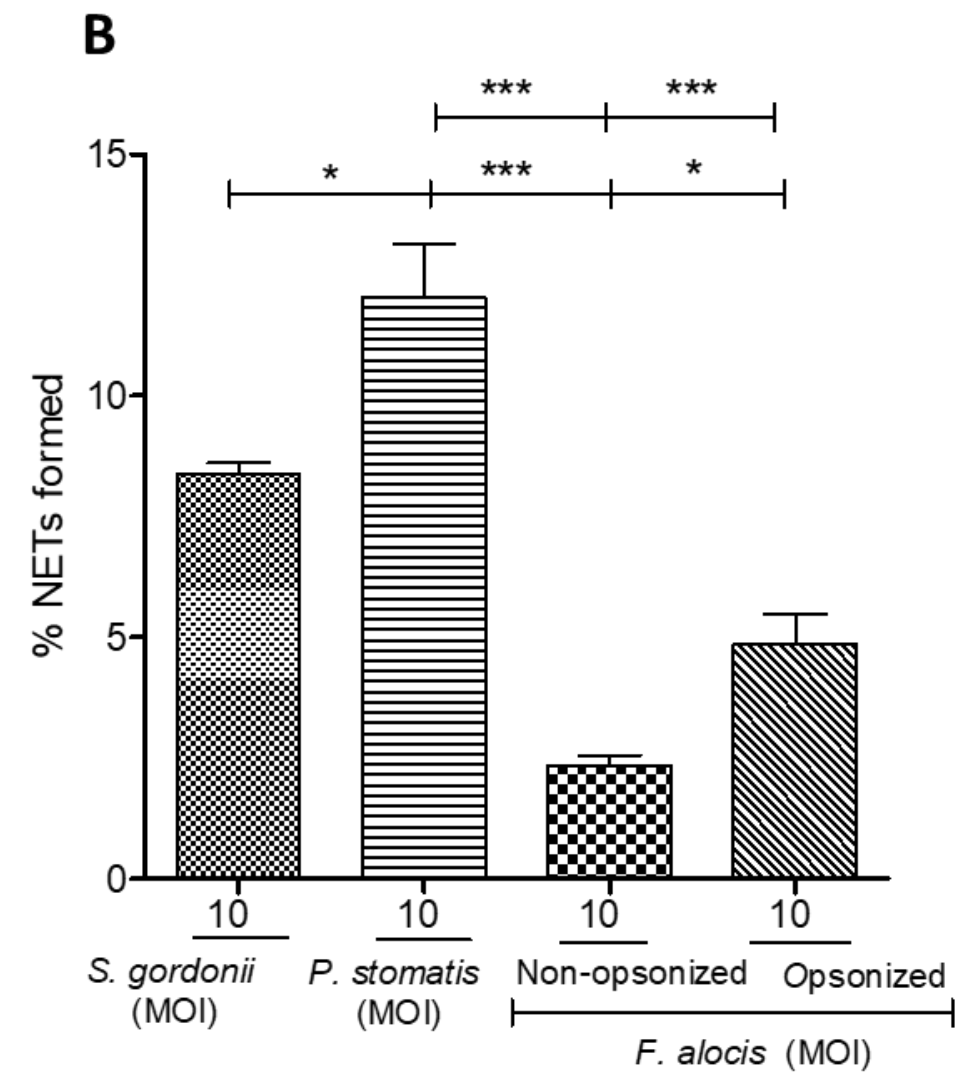


C

MOI (3 h)

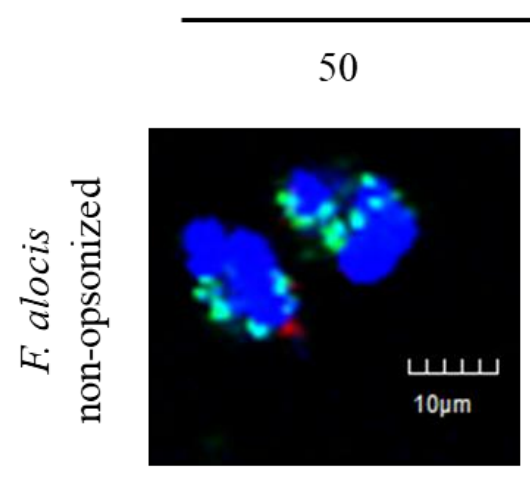

100
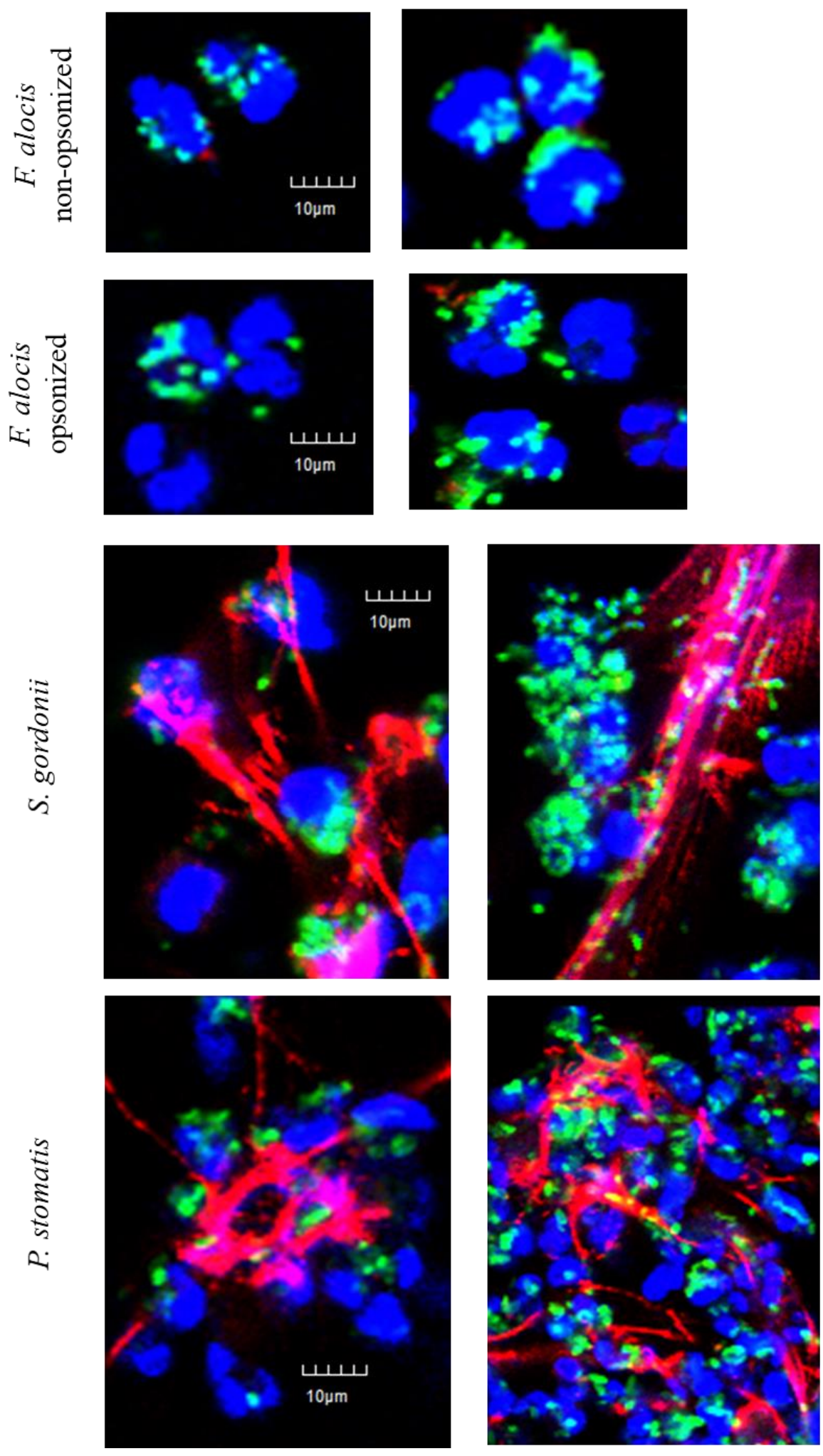


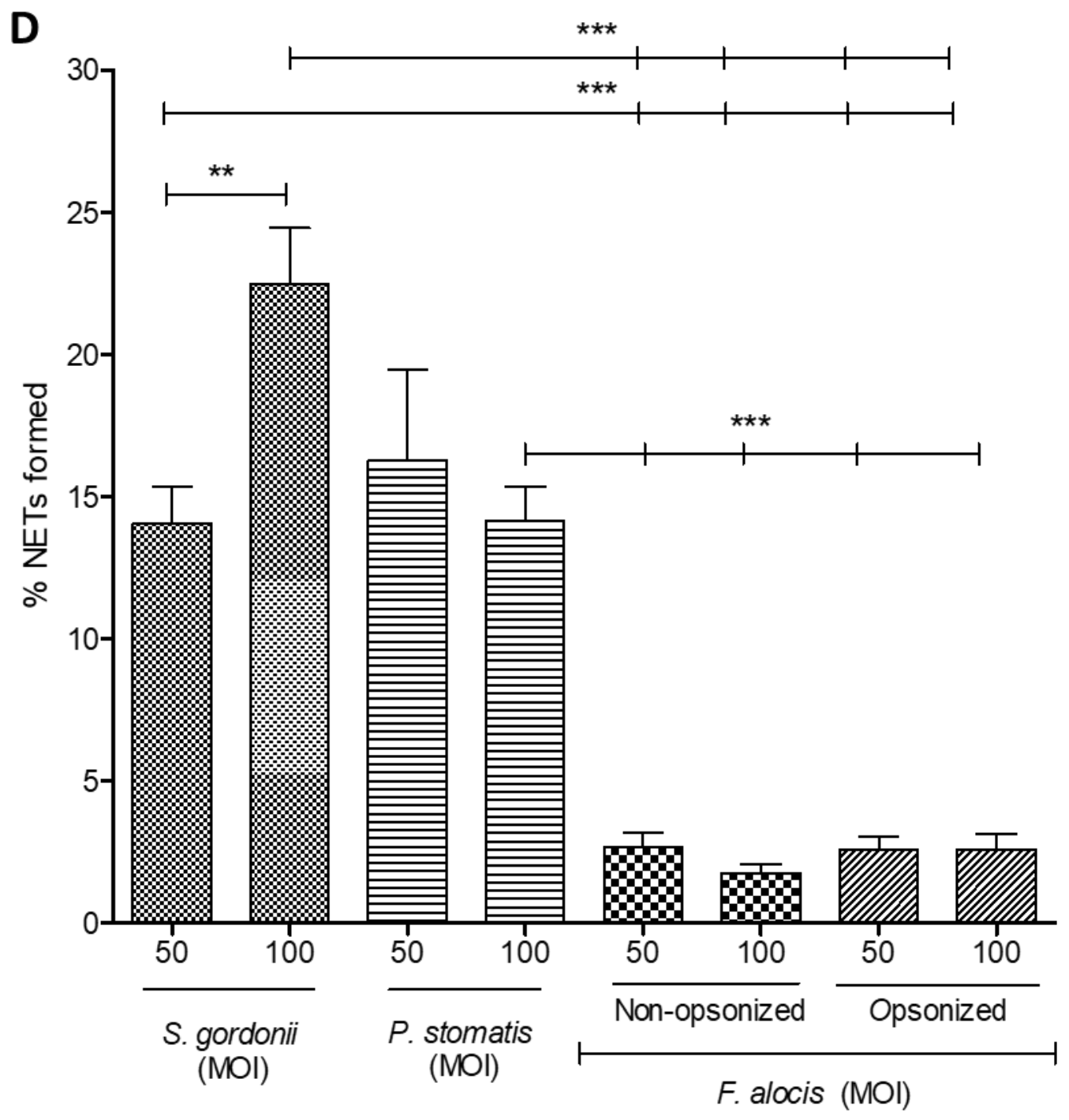

Figure 4-6. S. gordonii and P. stomatis induce significant NET formation in an MOIdependent manner.

Neutrophils were unchallenged (Basal), or challenged with CFSE-labeled S. gordonii (MOI 10/50/100), or CFSE-labeled P. stomatis (MOI 10/50/100), or CFSE-labeled non-opsonized F. alocis (MOI 10/50/100), or CFSE-labeled opsonized F. alocis (MOI 10/50/100) for $180 \mathrm{~min}$. Following infection, cells were fixed, exposed to antibodies directed against MPO (RedAlexaFluor647), stained with DAPI, and then imaged for NET immunofluorescence by confocal microscopy.(A) Representative confocal images (from 3 replicates of 100 quantified cells) of CFSE-labeled non-opsonized and opsonized $F$. alocis-challenged neutrophils and CFSE-labeled $S$. 
gordonii and CFSE-labeled P. stomatis at 180 min at MOI 10. (B) Quantification, using ImageJ analysis, of percentage of NETs formed from neutrophils challenged for 180 min with CFSElabeled S. gordonii (MOI 10), or CFSE-labeled P. stomatis (MOI 10), or CFSE-labeled nonopsonized F. alocis (MOI 10) or CFSE-labeled opsonized F. alocis (MOI 10). CFSE (green): bacteria, DAPI (blue): neutrophil nucleus/DNA, AlexaFluor647 (red): MPO, Merge: NET formation. Data are means $+/-$ SEM from 3 independent experiments. $* \mathrm{p}<0.05$, $* * * \mathrm{p}<0.001$. (C) Representative confocal images (from 3 replicates of 100 quantified cells) of CFSE-labeled nonopsonized and opsonized $F$. alocis-challenged neutrophils and CFSE-labeled $S$. gordonii and $P$. stomatis at $180 \mathrm{~min}$ at MOI 50 and MOI 100. (D) Quantification, using ImageJ analysis, of percentage of NETs formed from neutrophils challenged for $180 \mathrm{~min}$ with CFSE-labeled $S$. gordonii (MOI 50, 100), or CFSE-labeled P. stomatis (MOI 50, 100) or CFSE-labeled nonopsonized F. alocis (MOI 50, 100) or CFSE-labeled opsonized F. alocis (MOI 50, 100). Data are means $+/$ SEM from 3 independent experiments. ${ }^{* *} \mathrm{p}<0.01, * * * \mathrm{p}<0.001$. 

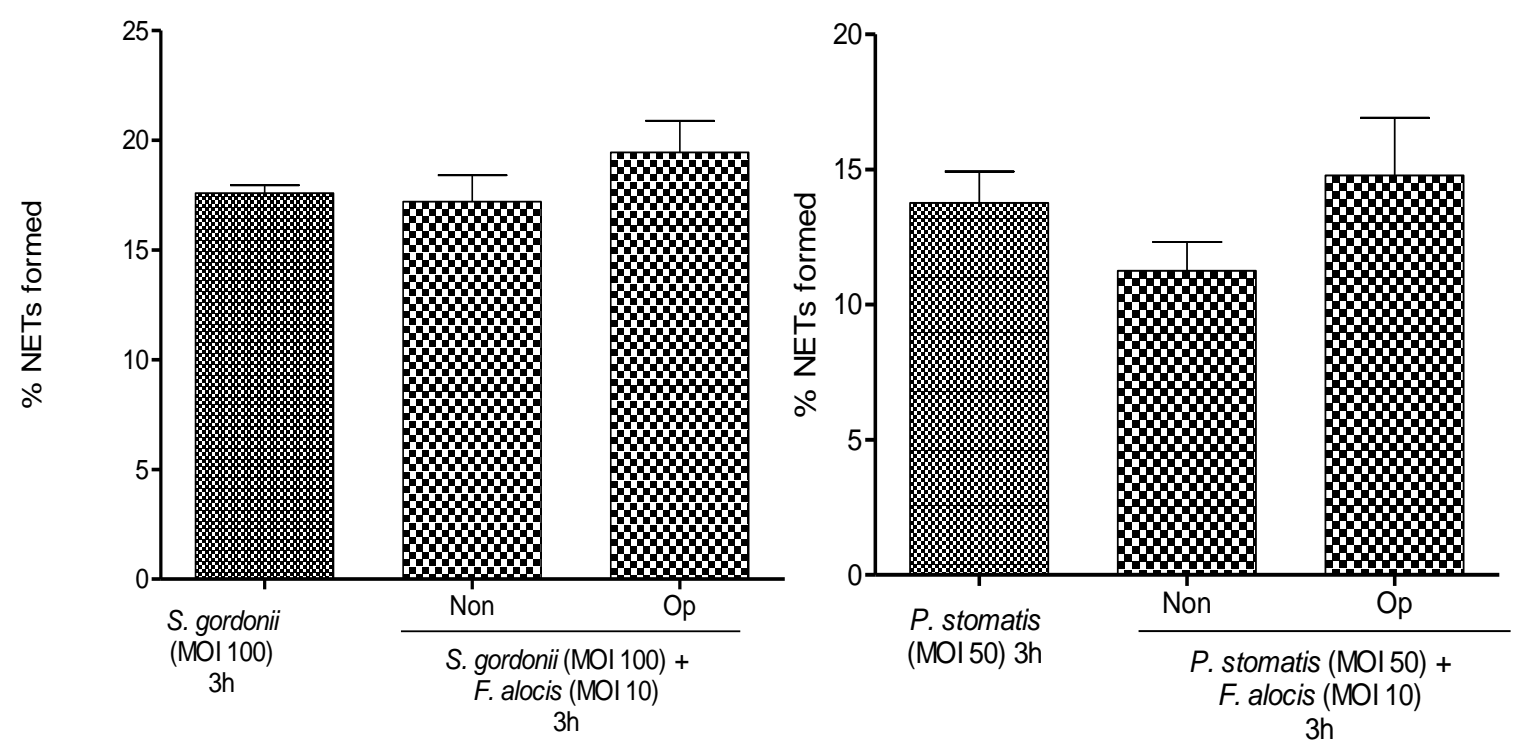

Figure 4-7. Coinfection of $F$. alocis with $S$. gordonii or $P$. stomatis does not reduce or exacerbate NET formation.

Neutrophils were challenged with HI-labeled S. gordonii (MOI 100), or with HI-labeled S. gordonii (MOI 100) and CFSE-labeled non-opsonized F. alocis (MOI 10) or CFSE-labeled opsonized F.alocis (MOI 10) for $180 \mathrm{~min}$ or challenged with HI-labeled P. stomatis (MOI 50), or with HI-labeled P. stomatis (MOI 50) and CFSE-labeled non-opsonized F. alocis (MOI 10) or CFSE-labeled opsonized F. alocis (MOI 10) for $180 \mathrm{~min}$. Following infection, cells were fixed, exposed to antibodies directed against MPO (Red- AlexaFluor647), stained with DAPI, and then imaged for NET immunofluorescence by confocal microscopy. (A) Quantification, using ImageJ analysis, of percentage of NETs formed from neutrophils challenged for $180 \mathrm{~min}$ with HI-labeled S. gordonii (MOI 100) and HI-labeled S. gordonii (MOI 100) and CFSE-labeled non-opsonized and opsonized F. alocis (MOI 10) for 180 min. Data are means +/- SEM from 3 independent experiments. (B) Quantification, using ImageJ analysis, of percentage of NETs formed from neutrophils challenged for 180 min with HI-labeled $P$. stomatis (MOI 50) and HI-labeled $P$. 
stomatis (MOI 50) and CFSE-labeled non-opsonized and opsonized F. alocis (MOI 10) for 180 min. Data are means +/- SEM from 3 independent experiments. 

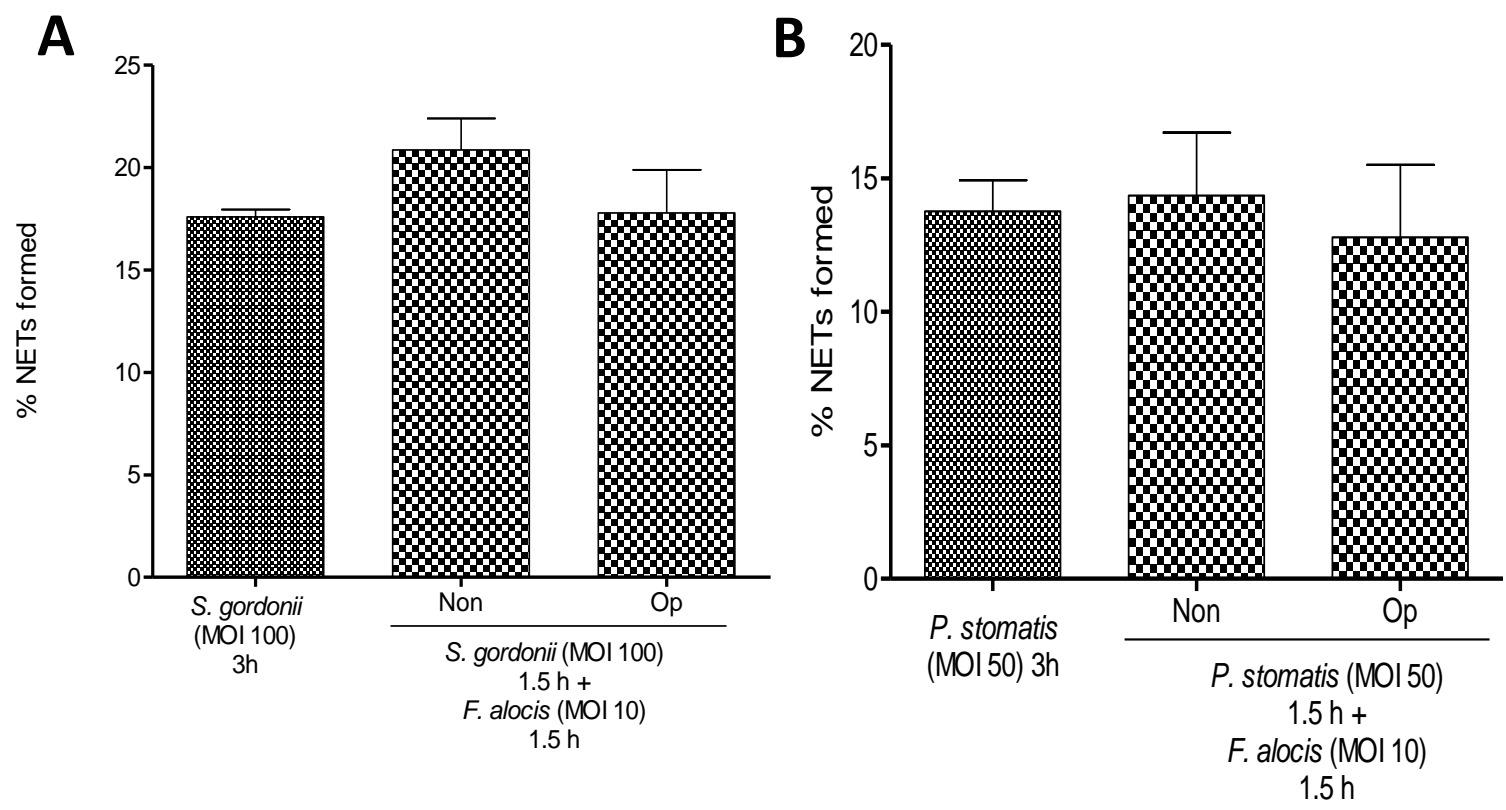

Figure 4-8. F. alocis fails to degrade NETs formed by $S$. gordonii and $P$. stomatis. Neutrophils were challenged with HI-labeled S. gordonii (MOI 100) for $180 \mathrm{~min}$, or HI-labeled $S$. gordonii (MOI 100) for $90 \mathrm{~min}$ and then with CFSE-labeled non-opsonized F. alocis (MOI 10) or CFSE-labeled opsonized F. alocis (MOI 10) for 90 min or challenged with HI-labeled P. stomatis (MOI 50) for $180 \mathrm{~min}$, or HI-labeled P. stomatis (MOI 50) for $90 \mathrm{~min}$ and then with CFSE-labeled non-opsonized F. alocis (MOI 10) or CFSE-labeled opsonized F. alocis (MOI 10) for $90 \mathrm{~min}$. Following infection, cells were fixed, exposed to antibodies directed against MPO (RedAlexaFluor647), stained with DAPI, and then imaged for NET immunofluorescence by confocal microscopy. (A) Quantification, using ImageJ analysis, of percentage of NETs formed from neutrophils challenged for 180 min with HI-labeled S. gordonii (MOI 100) and HI-labeled S. gordonii (MOI 100) for 90 min and then with CFSE-labeled non-opsonized F. alocis (MOI 10) or CFSE-labeled non-opsonized $F$. alocis (MOI 10) for 90 min. Data are means +/- SEM from 3 independent experiments. (B) Quantification, using ImageJ analysis, of percentage of NETs formed from neutrophils challenged for 180 min with HI-labeled P. stomatis (MOI 50) and HI-labeled $P$. stomatis (MOI 50) for 90 min and then with CFSE-labeled non-opsonized F. alocis (MOI 10) or 
CFSE-labeled non-opsonized F. alocis (MOI 10) for 90 min. Data are means +/- SEM from 3 independent experiments. 
A

PMA (3 h)

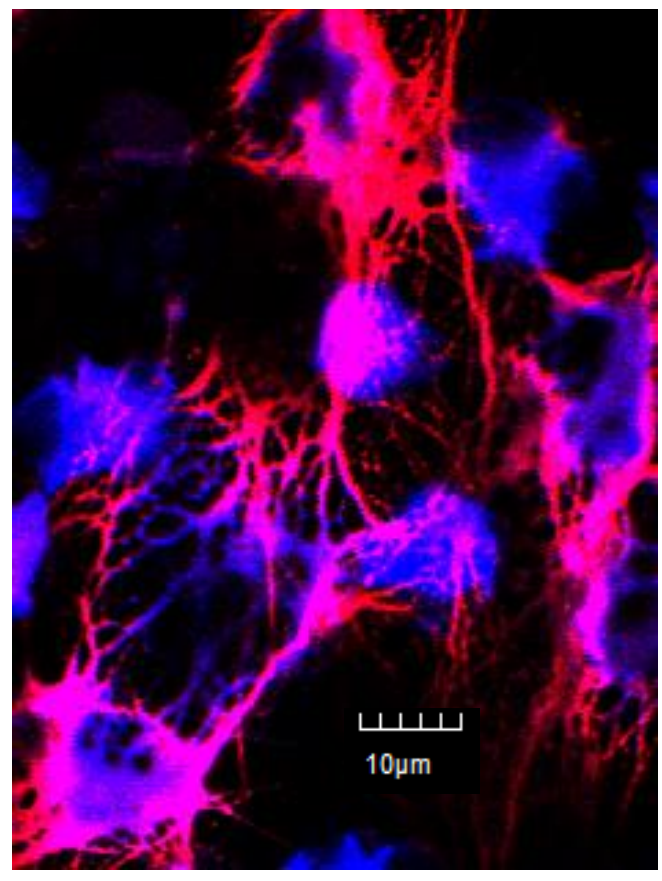

Non-opsonized $F$. alocis (60 min) + PMA (3 h)

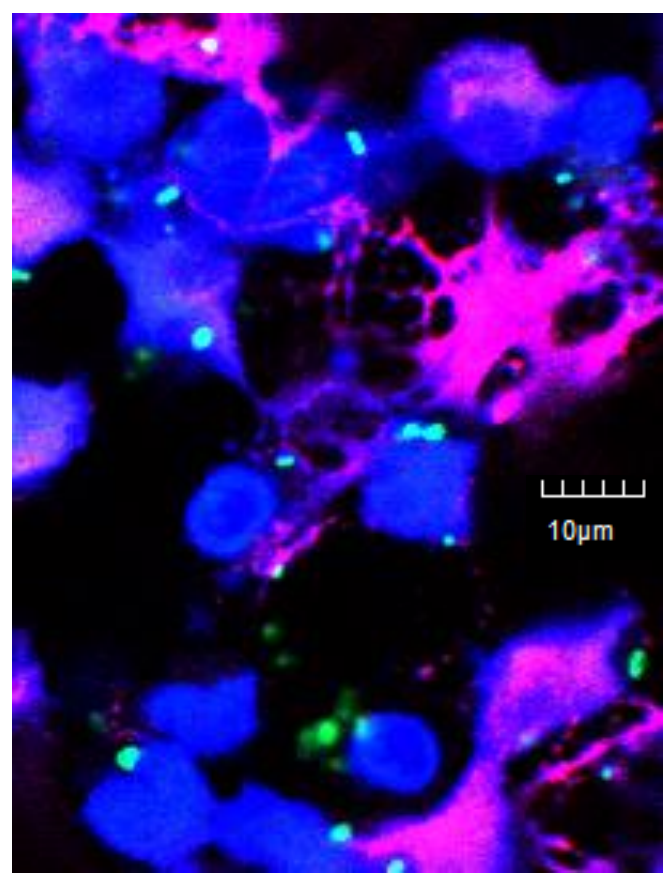


B

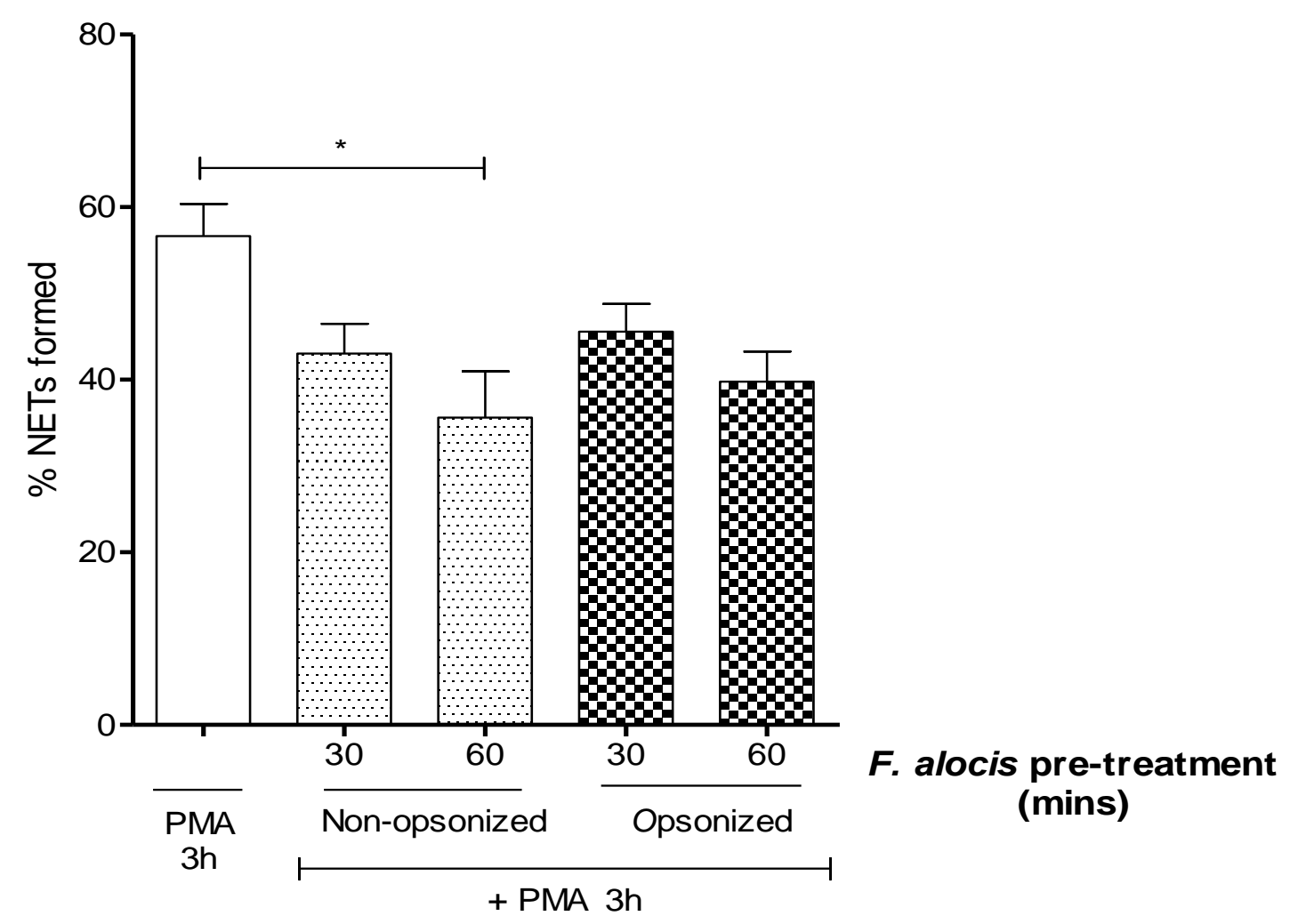

Figure 4-9. F. alocis pre-treatment can cause significant decrease in NET formation induced by PMA.

Neutrophils were challenged with PMA for $180 \mathrm{~min}$, or pre-treated with non-opsonized and opsonized CFSE-labeled F. alocis for 30-60 min and then exposed to PMA (180 min). Following infection, cells were fixed, exposed to antibodies directed against MPO (Red- AlexaFluor647), stained with DAPI, and then imaged for NET immunofluorescence by confocal microscopy. (A) Representative confocal images (from 3 replicates of 100 quantified cells) of neutrophils exposed to PMA (180 min) or pre-treated with non-opsonized CFSE- labeled F. alocis (60 min) before exposure to PMA (180 min). CFSE (green): bacteria, DAPI (blue): neutrophil nucleus/DNA, AlexaFluor647 (red): MPO, Merge: NET formation. (B) Quantification, using ImageJ analysis, of percentage of NETs formed from neutrophils challenged for 180 min with PMA and pre-treated 
with non-opsonized and opsonized CFSE-labeled F. alocis for 30-60 min and then exposed to PMA (180 min). Data are means +/- SEM from 4 independent experiments. ${ }^{*} \mathrm{p}<0.05$. 
A

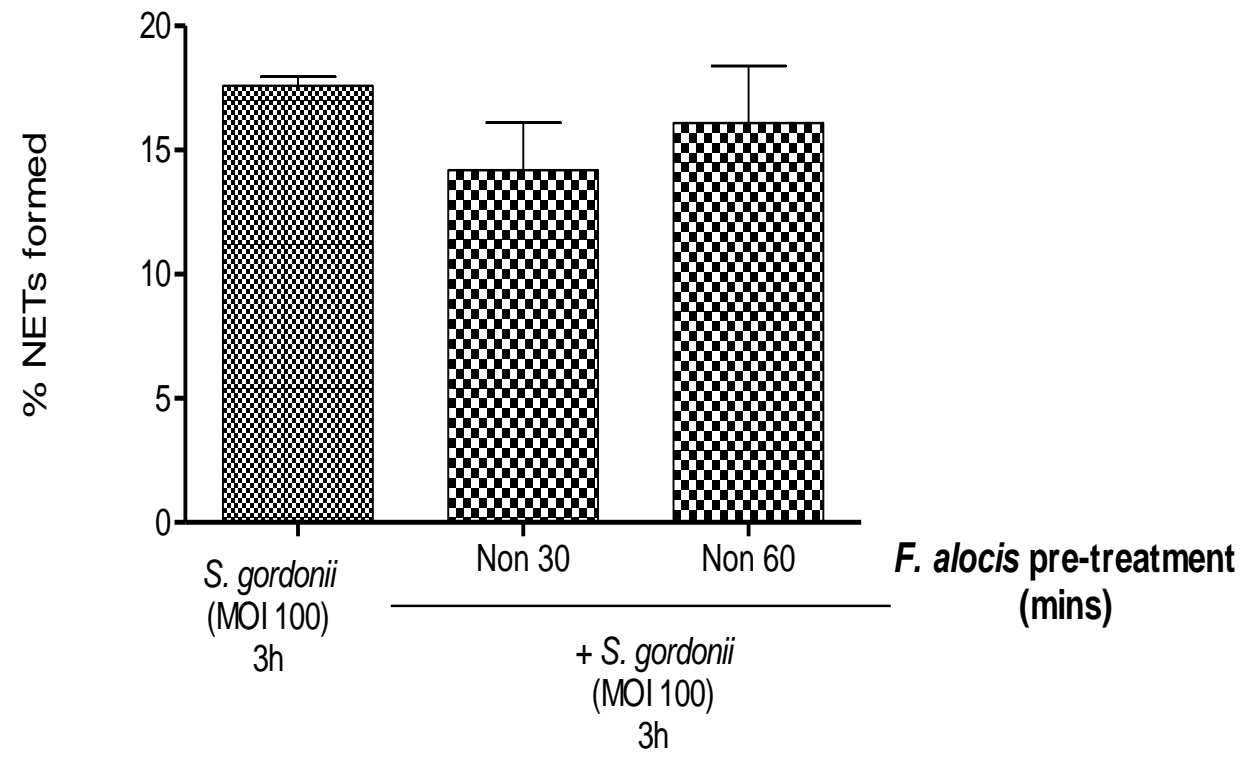

B

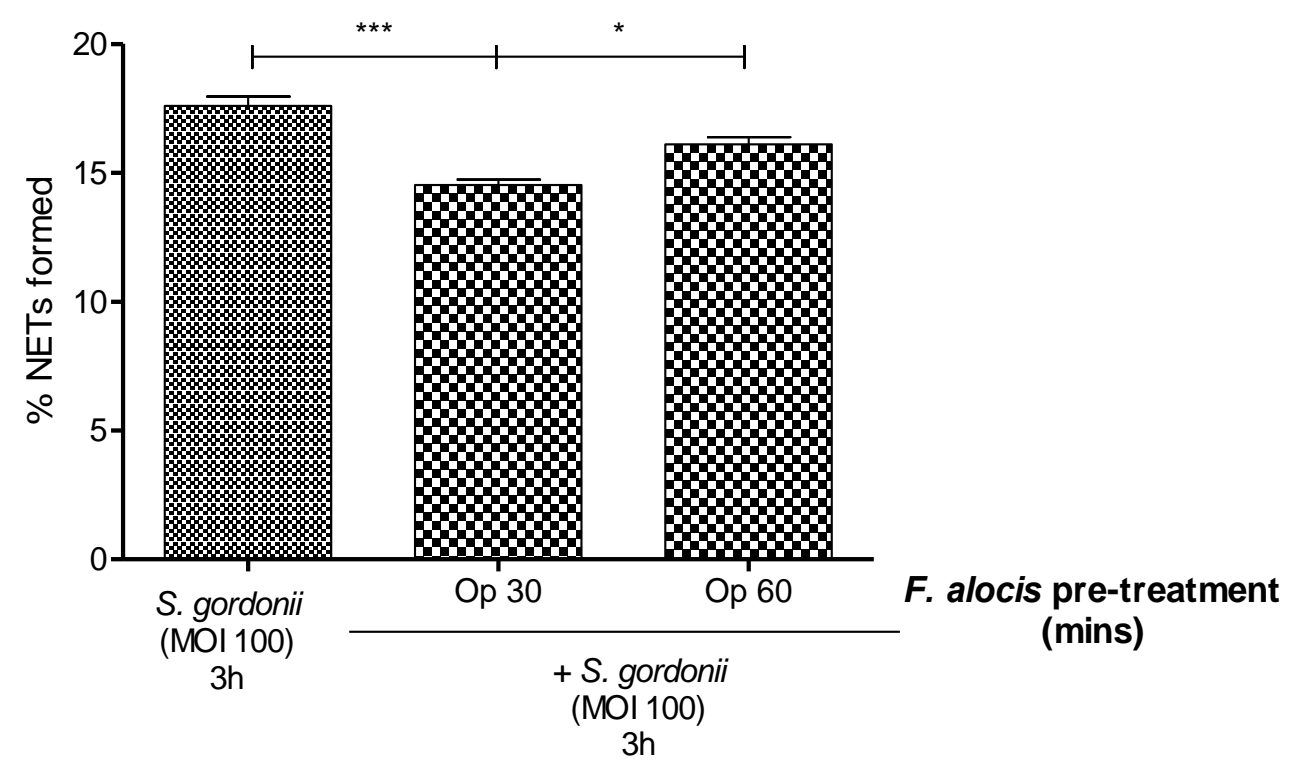



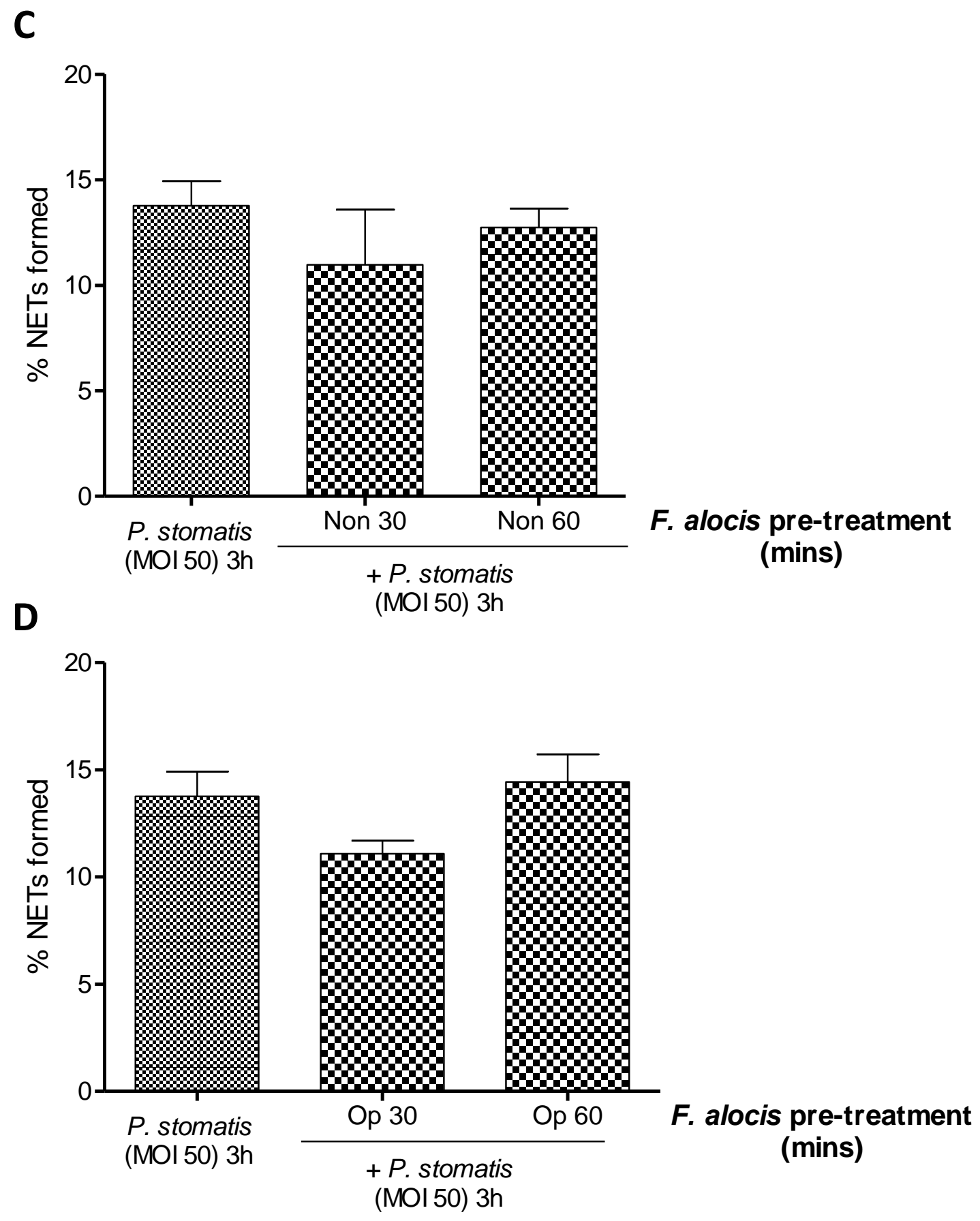

Figure 4-10. $F$. alocis pre-treatment significantly decreases NET formation induced by $S$. gordonii but not by $P$. stomatis.

Neutrophils were challenged with HI-labeled S. gordonii (MOI 100) for 180 min or HI-labeled $P$. stomatis (MOI 50) for $180 \mathrm{~min}$, or pre-treated with non-opsonized and opsonized CFSE-labeled F. alocis for 30-60 min and then challenged with HI-labeled S. gordonii (MOI 100) for $180 \mathrm{~min}$ 
or HI-labeled P. stomatis (MOI 50) for $180 \mathrm{~min}$. Following infection, cells were fixed, exposed to antibodies directed against MPO (Red- AlexaFluor647), stained with DAPI, and then imaged for NET immunofluorescence by confocal microscopy. (A) Quantification, using ImageJ analysis, of percentage of NETs formed from neutrophils challenged for 180 min with HI-labeled $S$. gordonii (MOI 100) or neutrophils pre-treated with non-opsonized CFSE-labeled F. alocis for 30-60 min and then challenged with HI-labeled S. gordonii (MOI 100) for 180 min. Data are means +/SEM from 3 independent experiments. (B) Quantification, using ImageJ analysis, of percentage of NETs formed from neutrophils challenged for $180 \mathrm{~min}$ with HI-labeled S. gordonii (MOI 100) or neutrophils pre-treated with opsonized CFSE-labeled $F$. alocis for 30-60 min and then challenged with HI-labeled S. gordonii (MOI 100) for 180 min. Data are means +/- SEM from 3 independent experiments. ${ }^{*} \mathrm{p}<0.05,{ }^{* * *} \mathrm{p}<0.001$. (C) Quantification, using ImageJ analysis, of percentage of NETs formed from neutrophils challenged for 180 min with HI-labeled P. stomatis (MOI 50) or neutrophils pre-treated with non-opsonized CFSE-labeled F. alocis for 30-60 min and then challenged with HI-labeled P. stomatis (MOI 50) for 180 min. Data are means +/- SEM from 3 independent experiments. (D) Quantification, using ImageJ analysis, of percentage of NETs formed from neutrophils challenged for 180 min with HI-labeled P. stomatis (MOI 50) or neutrophils pre-treated with opsonized CFSE-labeled F. alocis for 30-60 min and then challenged with HI-labeled P. stomatis (MOI 50) for 180 min. Data are means +/- SEM from 3 independent experiments. 


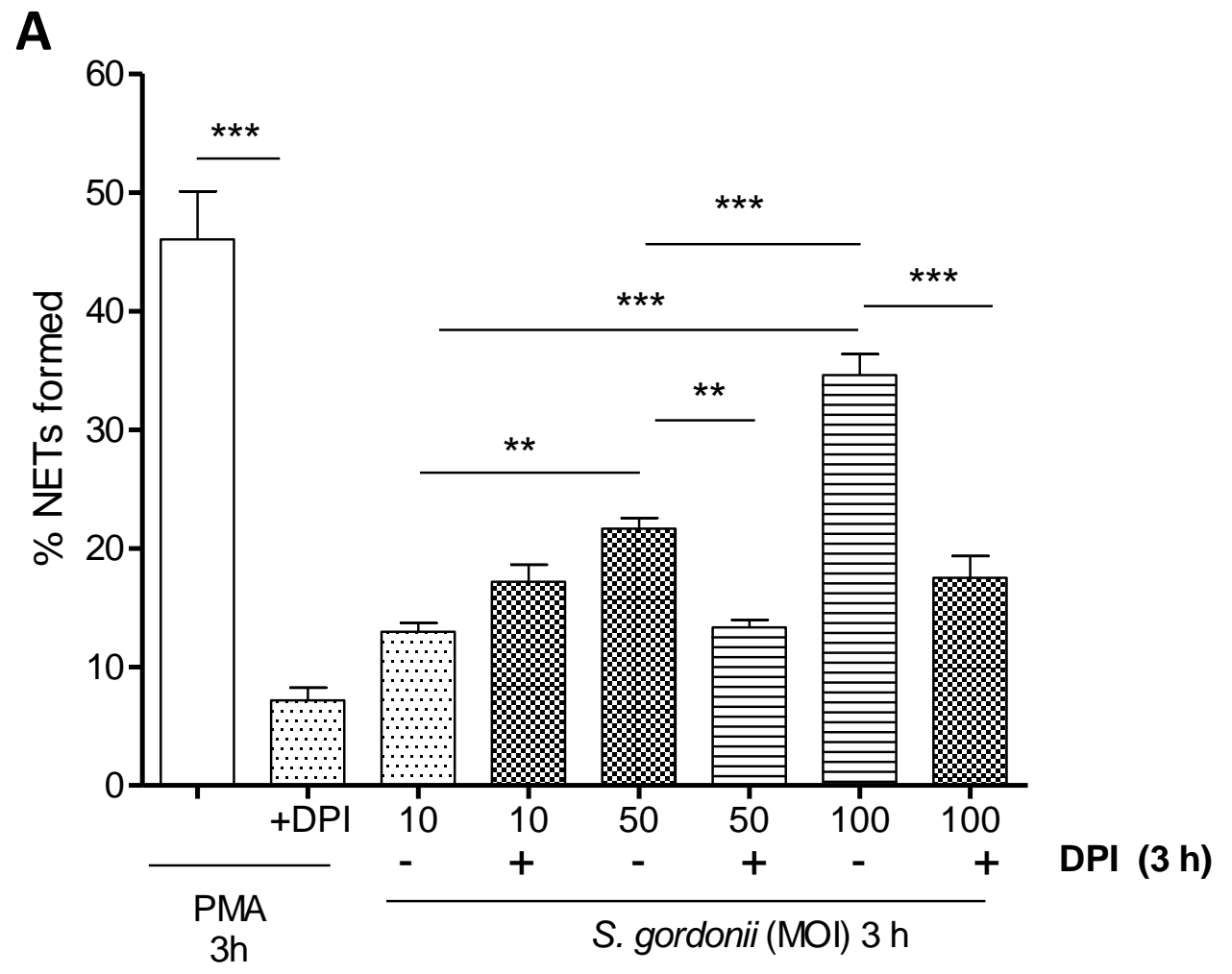

B

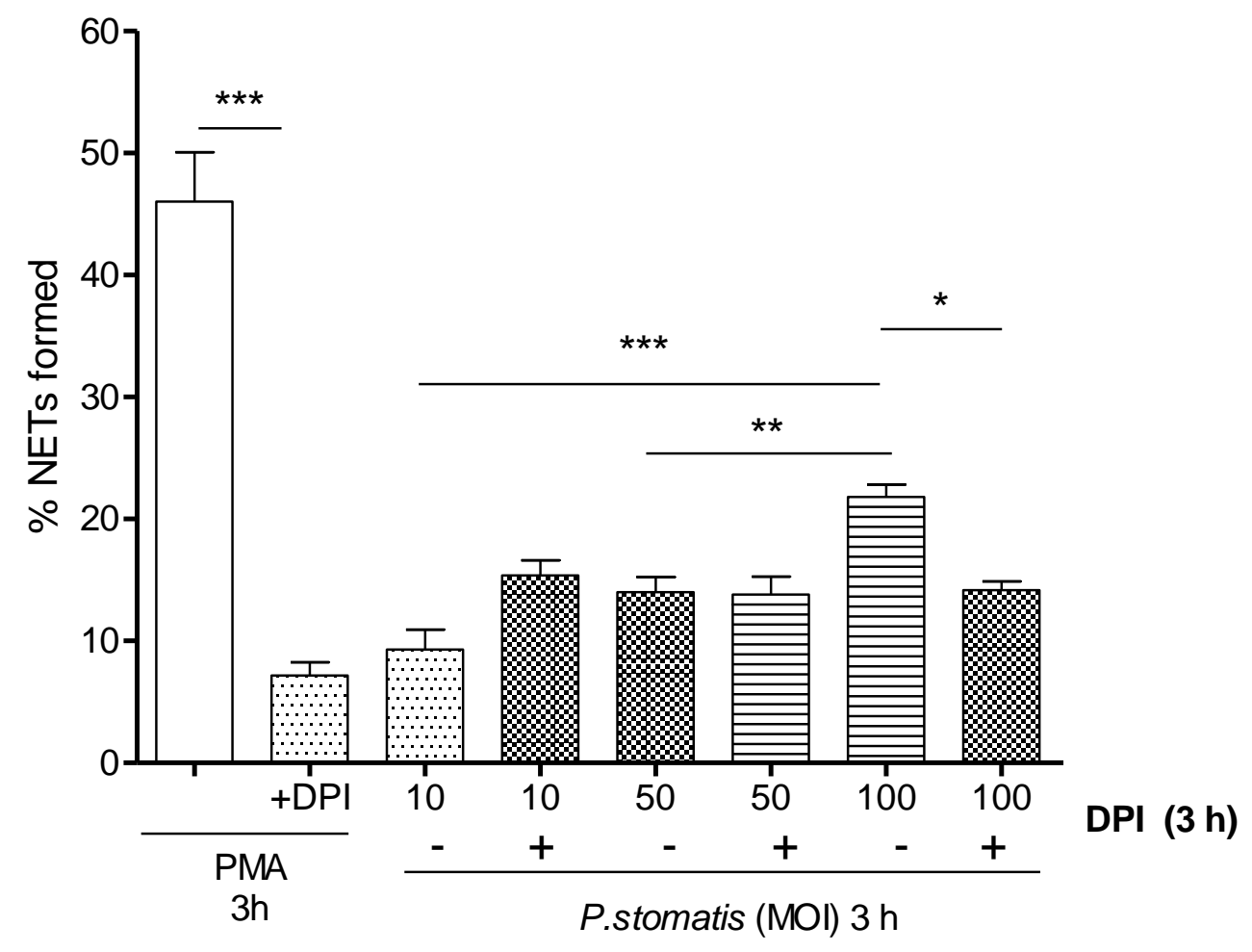


Figure 4-11. S. gordonii and $P$. stomatis induce NET formation in an MOI- and ROSdependent manner.

Neutrophils were challenged with CFSE-labeled $S$. gordonii (MOI 100) or CFSE-labeled $P$. stomatis (MOI 50) for $180 \mathrm{~min}$ or exposed to DPI $(10 \mu \mathrm{M})$ and then challenged with CFSElabeled S. gordonii (MOI 100) or CFSE-labeled P. stomatis (MOI 50) for 180 min. Following infection, cells were fixed, exposed to antibodies directed against MPO (Red- AlexaFluor647), stained with DAPI, and then imaged for NET immunofluorescence by confocal microscopy. (A) Quantification, using ImageJ analysis, of percentage of NETs formed from neutrophils challenged with CFSE-labeled S. gordonii (MOI 100) for 180 min or exposed to DPI (10 $\mu \mathrm{M})$ and then challenged with CFSE-labeled S. gordonii (MOI 100) for 180 min. Data are means +/SEM from 3 independent experiments. $* * p<0.01, * * * p<0.001$. (B) Quantification, using ImageJ analysis, of percentage of NETs formed from neutrophils challenged with CFSE-labeled $P$. stomatis (MOI 50) for 180 min or exposed to DPI $(10 \mu \mathrm{M})$ and then challenged with CFSElabeled P. stomatis (MOI 50) for 180 min. Data are means +/- SEM from 3 independent experiments. $* \mathrm{p}<0.05, * * \mathrm{p}<0.01, * * * \mathrm{p}<0.001$ 


\section{CHAPTER 5}

\section{GENERAL DISCUSSION}

Neutrophils, as professional phagocytes, are required to respond to a variety of stimuli that they encounter, which first begins with recognition and binding of the stimuli to receptors expressed on the plasma membrane of the cell. Given that $F$. alocis is a gram-positive organism, neutrophils recognize $F$. alocis through TLR2 receptors. Once receptor binding occurs, kinases (like ERK and p38 MAPK) are activated and further promote a variety of vital neutrophil functions such as cell activation, migration, and killing functions. Based on our data, it is clear that $F$. alocis triggers both ERK and $\mathrm{p} 38$ MAPK activation. We wanted to determine what neutrophil functions would be activated through TLR2-dependnet ERK and p38 MAPK signaling triggered by $F$. alocis challenge.

Once neutrophils are activated, they will begin the mobilization of their granules to the plasma membrane or to the phagosome, in order to ready themselves for further functions. Our data showed that $F$. alocis challenge induces significant granule exocytosis of both secretory vesicles and specific granules, further showing cell activation and stimulation of neutrophil functions. These functions are linked to the initial activation TLR2 and further downstream to p38 MAPK and ERK, as studies performed blocking either TLR2 or p38 MAPK significantly reduced granule exocytosis.

As the major cell type recruited to the periodontal pocket, it is important to first understand how neutrophils arrive at this site. Through responding to and deciphering signals from bacterial- and host- derived chemoattractant sources, neutrophils can perform directed 
migration to their target site. Perhaps, the most important chemokine for the context of the oral cavity and periodontal disease, is IL-8, as it is produced in large amounts at the site of infection. Additionally, it is the primary cytokine neutrophils respond to and also secrete themselves. Usually, oral pathogens will employ mechanisms to inhibit neutrophil chemotaxis to the periodontal pocket, as observed with periodontal pathogen, $P$. gingivalis, which generates chemokine paralysis by antagonizing the synthesis and release of IL-8 from gingival epithelial cells (267). In studies with $T$. denticola, it was observed that the bacterium effectively alters the balance of intracellular phosphoinositide, inhibits PI3-kinase activity, and increases phosphatase PTEN, which leads to inhibition of downstream signaling and compromised actin dynamics, which impair neutrophil chemotaxis (266).

However, we observe that $F$. alocis enhances chemotactic migration to IL-8. This strategy must benefit the bacteria in some way, as the increased presence of neutrophils would help to ensure chronic inflammation and nutrient-rich environment for $F$. alocis. In the in vivo setting, early colonizers of the oral community, like $S$. gordonii, may initiate neutrophil migration to the site of bacterial plaque formation on the gingival epithelium (411). However once $F$. alocis is introduced into the community, this bacterium could trigger the recruitment of additional neutrophils to the oral cavity; however, they will be impaired in reaching the target site of bacterial infection on the gingival epithelium, further leading to destructive neutrophil killing mechanisms targeted instead at the host tissue.

Additionally, our studies showed a preference in chemotactic migration to IL-8, an intermediary chemoattractant, over migration to $\mathrm{MLF}$, an end-target chemoattractant. This reveals that $F$. alocis influences the neutrophil's ability to determine the most preferential and end-stage target site for its function. Further, neutrophils may not reach the site of bacterial presence in the oral cavity, and pre-maturely employ their killing mechanisms, negatively impacting the host tissues without damaging the bacteria, protected at the end-stage site, the oral cavity. In the context of a bacterial community, F. alocis could ensure a nutrient-rich 
environment for other bacteria and allow survival and persistence of these pathogens in the oral cavity, as the neutrophils are not effectively migrating to this site.

Interestingly, we observed that $F$. alocis is able to induce chemokinetic, or random migration, as neutrophils challenged with $F$. alocis showed enhanced migration to negative control buffer. In the absence of chemoattractant signaling cues, it is expected that migration would cease. However, if $F$. alocis manipulates neutrophils to migrate in a chemokinetic manner, this could disrupt the patrolling function of neutrophils, whose role is involved in maintaining host homeostasis and ensuring host health. This ensures continual host tissue destruction and a beneficial environment for an invading pathogen to thrive. The viability of an organism can impact its own ability to function as well as impact its interactions with other cells and bacteria. Therefore, our studies with cell migration pointed to a role for heat-stable components being responsible for the manipulation of cell migration of neutrophils, given that there was no difference in neutrophil migration challenged with heat-killed compared with viable bacteria.

Based on the data observed in Chapter 2, it was determined that $F$. alocis is effectively recognized by neutrophils and further stimulates its functional capacities, like degranulation and cell migration. However, F. alocis impairs a vital function of neutrophils, deciphering chemoattractant cues, in order to ensure activated neutrophils are retained in the gingival crevice. It is tempting to speculate that these $F$. alocis-infected neutrophils won't be able to release their antimicrobial components at the site of the bacterial plaque on the gingival epithelium, their ideal targeted site. Instead, granule exocytosis will occur in the gingival tissue, providing significant host tissue destruction and persistence of bacteria in the gingival crevice. Furthermore, our data shows that neutrophils are induced to undergo chemokinesis upon challenge with $F$. alocis, which leads to neutrophils that would normally be patrolling the environment in a low-level inflammatory state with no chemoattractant cues, to be in an activated state, potentially being retained in the gingival crevice and promoting host tissue damage. Not only is F. alocis providing 
a safe haven for itself to colonize the gingival epithelium, but a niche for other oral bacteria, where they can colonize, persist and cause infection.

Given our data from studies on TLR signaling, we determined $F$. alocis signals through TLR2 and further it is more likely thorough the heterodimer TLR 2/6 than TLR 2/1. Following recognition of $F$. alocis, neutrophils will begin their activation process leading to the induction of their antimicrobial killing mechanisms. The intracellular respiratory burst production by neutrophils in response to challenge is critical for the killing of internalized bacteria or particulates into the phagosome. In our studies, F. alocis is effectively internalized and observed to reside in the phagosome, however a minimal intracellular respiratory burst response is induced.

Due to the low intracellular respiratory burst response produced by neutrophils challenged with $F$. alocis, we thought to assess the recruitment of the NADPH oxidase components to the bacterial-containing phagosome. Based on previous literature, we determined that performing recruitment studies across a time course would help to pinpoint the timing of assembly and activation of the complex to the $F$. alocis-containing phagosome, as this is the precursor step to the production of an efficient respiratory burst response. The role of the cytosolic component, $\mathrm{p} 40^{\text {phox }}$, has recently been characterized (412) and its recruitment seems to play a key role in phagosomal ROS production (412). Our data shows a differential recruitment of $\mathrm{p} 40^{\text {phox }}$ between live and heat-killed $F$. alocis at the 15 min challenge. However, by the 60 min time point, the recruitment of $\mathrm{p} 40^{\text {phox }}$ is no longer impaired to the viable $F$. alocis-containing phagosome, indicating a delay in assembly of this component. Therefore, we believe that viability may play a role in the ability of the bacterium to manipulate neutrophil activation and assembly of the oxidase complex. Additionally, we can speculate that this component's recruitment plays an important role in the context of our studies, and it may be a crucial positive regulator of oxidative burst induction.

Perhaps the most studied of the NADPH oxidase components is $\mathrm{p} 47^{\text {phox }}$. In our studies, $\mathrm{p} 47^{\text {phox }}$ is recruited effectively to both the viable and heat-killed F. alocis-containing phagosome 
at the early time points, however at 60 mins, the component starts to come off only from the viable $F$. alocis-containing phagosome, which may indicate the time point when the oxidase is deactivated. Similarly, to what was observed with $\mathrm{p} 40^{\text {phox }}$, viability of the bacterium is playing a role in the detachment of $\mathrm{p} 47^{\text {phox }}$.

Rac activation and recruitment to the phagosome is known to be another important step in superoxide production and is linked to many pathways downstream of the NADPH oxidase assembly and activation. The active GTP-bound form of Rac is crucial for the regulation and activation of the NADPH oxidase complex, as was determined with mice defective in Rac-2. Although the Rac2 activation is linked to several neutrophil functions, in our studies, it is important to note that a deficient Rac2 activation leads to a deficient superoxide production. Our data revealed viable $F$. alocis fails to effectively activate Rac2 by neutrophils, which is not observed with the heat-killed $F$. alocis, further allowing for speculation that the viable bacterium impairs Rac activation, in order to evade neutrophil oxidative-mediated killing.

We can further speculate that there is an impairment in mechanisms involved in ROS production, like electron transfer, that can result in an impaired respiratory burst response, despite proper NADPH oxidase complex assembly. We know that heat-killed $F$. alocis induces a robust intracellular respiratory burst response, which could be explained by the retention of both $\mathrm{p} 47^{\text {phox }}$ and $\mathrm{p} 40^{\text {phox }}$ to the phagosomal compartment. The viable $F$. alocis induces a minimal intracellular respiratory burst response, however it effectively initiates the recruitment of both $\mathrm{p} 47^{\text {phox }}$ and $\mathrm{p} 40^{\text {phox }}$, with disassembly only occurring at a late time point with $\mathrm{p} 47^{\text {phox }}$. It is yet to be determined whether $\mathrm{p} 40^{\text {phox }}$ binds the PI3P site, which is known to be crucial for effective intracellular ROS production.

Another crucial mechanism in producing a potent intracellular ROS response is the recruitment of neutrophil granules to the bacterial-containing phagosome. Data performed in our laboratory showed that azurophilic granules, which contain potent antimicrobial component, myeloperoxidase (MPO), were impaired in their recruitment to the bacterial-containing 
phagosome. MPO is needed for the production of $\mathrm{HOCl}$, which is highly effective at killing microbes inside the neutrophil phagosome. Additionally, the specific granules, which contain cytochrome b558 (composed of cytosolic NADPH oxidase components gp9 $1^{\text {phox }}$ and $\mathrm{p} 22^{\text {phox }}$ ) and antimicrobial compounds lactoferrin and lysosome, are not effectively recruited to the $F$. alociscontaining phagosome. This could help to explain $F$. alocis ability to evade neutrophil oxidativebased intracellular killing mechanisms.

For those stimuli that are not effectively internalized into a phagosomal compartment, an extracellular respiratory burst response is induced, which is beneficial to ensure killing of extracellular stimuli, but can also serve detrimental to the host. Based on our studies, it appears that $F$. alocis does not trigger induction of this response, however it can pre-activate or prime the neutrophils to secondary stimuli. In the oral cavity, F. alocis could be priming neutrophils to have a more robust superoxide production, which could result in serious damage to the host tissues, allowing for periodontal disease progression through chronic inflammation.

Now that we understand how $F$. alocis challenge impacts the induction of the intracellular and extracellular respiratory burst response, it is necessary to evaluate the survival of the bacterium. We observed that $F$. alocis is able to remain viable both intracellularly and extracellularly up to $4 \mathrm{~h}$ post neutrophil challenge. We can speculate that the bacteria remain viable intracellularly due to the minimal intracellular respiratory burst response produced in the F. alocis-containing phagosome. Considering the percentage of $F$. alocis that remains viable extracellularly, this indicates that neutrophil superoxide production is ineffective at killing the bacteria, and instead these highly potent superoxide radicals are produced and provide extensive collateral damage to the host.

As seen with our data thus far, F. alocis challenge of neutrophils results in their activation; however, their functional responses and killing mechanisms are impaired. We sought to determine if NETs, would be induced by neutrophils challenged with $F$. alocis. NETs have been discovered in gingival epithelia and in oral bacteria biofilms. Our bacterium fails to induce 
NETs, indicating that $F$. alocis manipulates the cells to inhibit NETs from forming or actively degrades NETs. The release of extracellular neutrophil elastase has been implicated to be involved in the formation of NETs, therefore in the case of $F$. alocis challenge, we wanted to determine if extracellular NE could be detected. As anticipated based on our confocal immunofluorescence studies, $F$. alocis challenge failed to produce extracellular NE. This could point to a reason why $F$. alocis fails to induce neutrophils to form NETs.

It is clear that $F$. alocis may be unique to the oral bacteria community, as we saw neutrophils produced NETs in response to other oral bacteria, S. gordonii and P. stomatis. This also led us to speculate that NETs do play an important role in the context of the oral community, as two common bacteria found in the gingival epithelium, S. gordonii, a commensal organism, and $P$. stomatis, a pathogenic organism, are capable of inducing NETs. In the context of periodontal disease, it is possible that $F$. alocis can manipulate neutrophils from further induction of NETs upon encounter of oral bacteria that will stimulate their production. Additionally, it may be that $F$. alocis behaves self-sufficiently and only evades and fails to induce NETs, however it does not provide benefit to other members of the community that are capable of inducing NETs, which neutrophils will use to effectively trap and kill their target.

Overall, our studies on NET formation led us to speculate that $F$. alocis is not capable of inducing NETs, but can manipulate cells to impair their ability to form NETs. F. alocis proves to be able to evade yet another potential neutrophil killing mechanism, NET formation, through a yet-to-be-determined mechanism. As F. alocis is effectively internalized into a bacterialcontaining phagosome, neutrophils may not benefit from employing an extracellular-killing mechanism like NETs, as a means to combat $F$. alocis challenge. Additionally, NETs have the potential to trap and kill bacteria, so their reduction in the oral cavity with $F$. alocis presence can ensure oral bacteria persist and survive. As NETs are shown to block the gingival epithelium to prevent colonization of an oral bacteria biofilm, F. alocis could be providing benefit to the entire 
community, allowing for excessive bacterial plaque formation, and continual recruitment of neutrophils, chronic inflammation and further periodontal disease development. 


\section{CHAPTER 6 \\ CONCLUSION AND FUTURE STUDIES}

\section{F. alocis challenge promotes activation of neutrophil kinase signaling pathways, leading to enhanced cell migration and degranulation}

Neutrophils are vital first responder cells and found in abundance in the periodontal pocket in response to oral bacteria, their targeted site of activation and killing, in order to ensure host health and homeostasis $(179,198)$. In order to get to the site of infection, they must decipher through a bombardment of chemoattract cues, both intermediary (IL-8) and end-target (like fMLF) $(118,120,123)$. It is known that IL-8 is a chemotactic source found in high concentrations in the gingival crevice, therefore we utilized this as our intermediary chemoattractant source. It has been determined that neutrophils sampled from patients with chronic periodontitis have defective chemotactic migration capabilities (189), therefore, we wanted to determine neutrophil migration in the context of $F$. alocis challenge.

When challenging neutrophils with $F$. alocis, we observed chemokinetic migration toward negative control buffer and an enhanced chemotactic migration towards IL-8, but not fMLF. Viability did not impair the bacterium's ability to impact cell migration, as a similar trend was seen with challenge of heat-killed $F$. alocis. In order to determine how $F$. alocis-challenged neutrophils would respond to a choice of IL-8 or fMLF in comparison with unchallenged neutrophils, it would be necessary to perform under-agarose migration assays, which allow for study of cells exposed to multiple chemotactic sources in different spatial and temporal combinations (413).

F. alocis, a gram-positive bacterium, is recognized by neutrophils through TLR2 (81) to further activate p38 MAPK and ERK kinase signaling pathways. In our studies, we determined 
that $F$. alocis induced both $\mathrm{p} 38$ MAPK and ERK activation, with the maximum activation at 30 mins that decreased by $60 \mathrm{~min}$. The necessity for TLR 2 recognition in further initiating kinase signaling was shown through blocking TLR2 and seeing a decrease in kinase phosphorylation.

TLR signaling can also induce a variety of neutrophil functions including granule exocytosis, which may also play a role in chemotaxis $(81,249,252,414)$. We observed that $F$. alocis is capable of inducing both secretory vesicle and specific granule exocytosis, which was TLR2 and p38 MAPK dependent. To further delineate the potential role granule exocytosis plays in neutrophil chemotaxis, we utilized a granule exocytosis inhibitor, TAT-SNAP-23 (146), and then performed cell migration studies. When blocking granule exocytosis, we saw a significant inhibition in both chemokinesis and chemotaxis of neutrophils challenged with F. alocis.

It would be important to further determine how neutrophil migration is impacted in the context of periodontal disease, which would be performed by challenging neutrophils with multiple oral bacteria, such as $P$. gingivalis and $P$. stomatis and quantify transwell migration after exposure to buffer, fMLF or IL-8. It would be necessary to determine if random and directed cell migration were impacted similarly, as observed in our studies with F. alocis.

\section{Strategies employed by $F$. alocis to ensure minimal respiratory burst production by neutrophils and mediate their survival from oxidative killing mechanisms}

We determined TLR2 signaling is responsible for recognition of $F$. alocis by neutrophils, however it is important to determine if this signaling is through the TLR2/1 or TLR 2/6 heterodimer. Using known TLR 2/1 agonist (PAM3CSK4) and TLR 2/6 agonist (FSL-1), we were able to show that $F$. alocis challenge of neutrophils behaved similarly in its induction of superoxide production as FSL-1.

Our studies into further deciphering the TLR 2/6 signaling require further experiments utilizing TLR 1 and TLR 6 neutralizing antibodies to determine if there is an impairment in the superoxide production of neutrophils exposed to neutralizing antibodies before $F$. alocis 
challenge. Additionally, we will use TLR blocking antibodies to determine how the superoxide production is affected if TLR 2 or TLR 6 is unable to recognize and bind $F$. alocis.

Given the low intracellular respiratory burst response we saw induced by neutrophils challenged with viable $F$. alocis, we sought to characterize the assembly of the NADPH oxidase complex to the bacteria-containing phagosome, as this is a crucial pre-cursor step to producing a sufficient intracellular respiratory burst response (143). However, we observed similar recruitment of $\mathrm{p} 47^{\text {phox }}, \mathrm{p} 67^{\text {phox }}$, gp9 $1^{\text {phox }}$ and $\mathrm{p} 22^{\text {phox }}$ by 15 mins to both viable and heat-killed $F$. alocis-containing phagosomes. The only difference in recruitment was seen with $\mathrm{p} 40^{\text {phox }}$ at an early time point but that difference was lost by 30 and 60 min post infection. This indicates that there is a delay in recruitment of the $\mathrm{p} 40^{\text {phox }}$ to the phagosome, which could explain the impaired intracellular respiratory burst response. Additionally, Rac activation, was impaired at 30 min with viable $F$. alocis challenge compared to heat-killed $F$. alocis, indicating that the viable bacterium is able to manipulate the Rac activation status and further impact the magnitude of the respiratory burst response produced by neutrophils.

Additionally, as it has been determined that the oxidase components are in a dynamic 'On-Off' state, it should be determined through time course studies with live-cell imaging if and when $F$. alocis manipulates the recruitment of the NADPH oxidase components. For these studies, we will utilize neutrophil differentiated PLB-985 cells with stable expression of yellow fluorescent protein (YFP)-tagged p6 $7^{\text {phox }}$, YFP-tagged p47 $7^{\text {phox }}$., and mCherry-tagged p40PX (142, $324)$.

Although impaired translocation of the NADPH oxidase components may not be the factor causing a low intracellular burst response, it will be important to also look at the phosphorylation status of the components, as their activation is critical for their functional capabilities $(190,356)$. To help to further characterize the NADPH oxidase assembly and activation and its role in the iROS response produced by neutrophils challenged with $F$. alocis, it will be necessary to perform Western blotting of the phosphorylated components to determine if 
they are recruited effectively, but remain enzymatically inactive. As our data only revealed the disassembly of $47^{\text {phox }}$ by 60 mins, future studies with the remaining components of the oxidase $\left(\mathrm{p} 40^{\text {phox }}, \mathrm{p} 67^{\text {phox }}, \mathrm{gp} 91^{\text {phox }}, \mathrm{p} 22^{\text {phox }}\right.$ ) are needed to determine when their disassembly occurs.

We observed that $F$. alocis remained viable both intracellularly $(\sim 65 \%)$ and extracellularly ( $40 \%)$, up to $4 \mathrm{~h}$ post-neutrophil challenge. Furthermore, F. alocis challenge induced minimal ROS production. In order to determine if oxidants are required for bacteria killing if introduced into the system exogenously, we will utilize the glucose-glucose oxidase system $(146,415)$. To do so, neutrophils will be pre-incubated with glucose plus glucose-oxidase and then challenged with $F$. alocis and BacLight viability staining will be applied to assess internal and external viability of the bacteria. If $F$. alocis is effectively killed, this would lead us to determine that the activation of the oxidase is important to for neutrophil killing in our studies. Additionally, as $F$. alocis possesses sialidase and also superoxide reductase, it may be of benefit to inhibit these enzymes using neuraminidase or a sialidase inhibitor and determining if the intracellular ROS is produced. Future studies should help determine if and at what time point $F$. alocis is effectively killed by the neutrophils, therefore performing this experiment with a later time point challenge would be necessary.

Lastly, in order to better characterize the neutrophil response in the context of periodontal disease, it would be beneficial to perform co-infection or multi-species studies with $F$. alocis and other oral microbes to determine if the bacterium overall can impact the community and the effectiveness of the neutrophil killing mechanisms. Additionally, pre-treating the neutrophils with F. alocis and then introducing another oral microbe or multispecies community would help to determine if the oxidative killing mechanisms can be inhibited by our bacterium. From a therapeutic standpoint, it is possible that the augmentation of enzymes that protect against oxidative stress could serve beneficial for treatment of periodontitis (286).

F. alocis fails to induce NETs and further manipulates the neutrophil's capacity to induce NETs in response to both pharmacological and bacterial stimuli 
NETs are known to be induced from neutrophils in the oral cavity when they encounter oral pathogens $(197,367)$, however, it was yet-to-be-determined whether $F$. alocis is capable of inducing NETs. In our time course studies, we observed that viable $F$. alocis (non-opsonized and opsonized) and heat-killed $F$. alocis fail to induce NETs. Additionally, increasing the MOI of $F$. alocis did not cause NET induction. However, when we challenged neutrophils with two other oral bacteria, S. gordonii and P. stomatis, we saw there was significant NET induction and in the case of $S$. gordonii the NETs were induced in an MOI-dependent manner.

To attempt to mimic a more physiologically relevant environment to the oral cavity, we performed co-infection studies with $F$. alocis and S. gordonii or P. stomatis. While F. alocis was incapable of reducing or exacerbating NET formation when in co-infection with $S$. gordonii or $P$. stomatis and the bacterium could not degrade pre-formed NETs induced by $S$. gordonii or $P$. stomatis, we observed that $F$. alocis is capable of manipulating neutrophils to impair their ability to form NETs when challenged with inducers like S. gordonii and PMA. As the biofilm community is the natural environment for the oral cavity, it will be important to determine how $F$. alocis behaves in this context by exposing neutrophils to three or four species biofilms.

Future studies should try to determine if NETs that are formed have antibacterial properties against $S$. gordonii or $P$. stomatis, and can effectively kill the bacteria. The best approach would be to perform survival studies using the BacLight viability assay. Additionally, to determine if NETs are the preferred killing method employed by neutrophils, studies will be performed where neutrophils will be exposed to actin-disrupting drugs (Latrunculin A or Cytochalasin D), which inhibit phagocytic-based killing mechanisms and then challenged with either S. gordonii or P. stomatis to see if the NET formation is reduced or exacerbated (151).

Future studies are needed to further characterize the signaling mechanisms that $F$. alocis may manipulate in order to inhibit NET formation. The NET signaling pathway is complex and 
has steps/stages that must occur that precede he extracellular extrusion of DNA, such as stimulation of receptors, Raf/MEK/ERK pathway activation, assembly of NADPH oxidase complex, ROS production, intracellular membrane disintegration, granular protein NE enters the nucleus and processes core histones, hypercitrullination and decondensation/mobilization of chromatin $(168,406)$. To do so, Western blotting studies will be performed to determine the activation or expression of Raf, MEK, PKC, PAD4, PI3K, Akt and mTOR $(168,365,384,406$, 416-418).

Lastly, as NETs are known to be formed in the oral cavity, it will be important to determine if NETs are present, if they have antimicrobial properties against $F$. alocis. Neutrophils will be stimulated with PMA, S. gordonii or P. stomatis to induce NETs and these isolated NETs will be introduced to an $F$. alocis challenge setting and BacLight viability staining will be used to determine if it has antimicrobial properties towards $F$. alocis (165).

Overall, the work presented in this dissertation is the first study of the oral pathogen, Filifactor alocis, and human neutrophils. A diagram summarizing our findings is depicted in Fig. 6-1. Given the fact that the presence of F. alocis in the oral cavity is indicative of periodontal disease, it is crucial to further study this bacterium for use as a potential biomarker. Periodontal disease results from host and bacterial-derived factors, further implicating the importance of knowing how the neutrophils are manipulated by F. alocis, as they are the key cell recruited to the gingival epithelium and known to promote disease if retained in the host tissues inducing a state of chronic inflammation. 


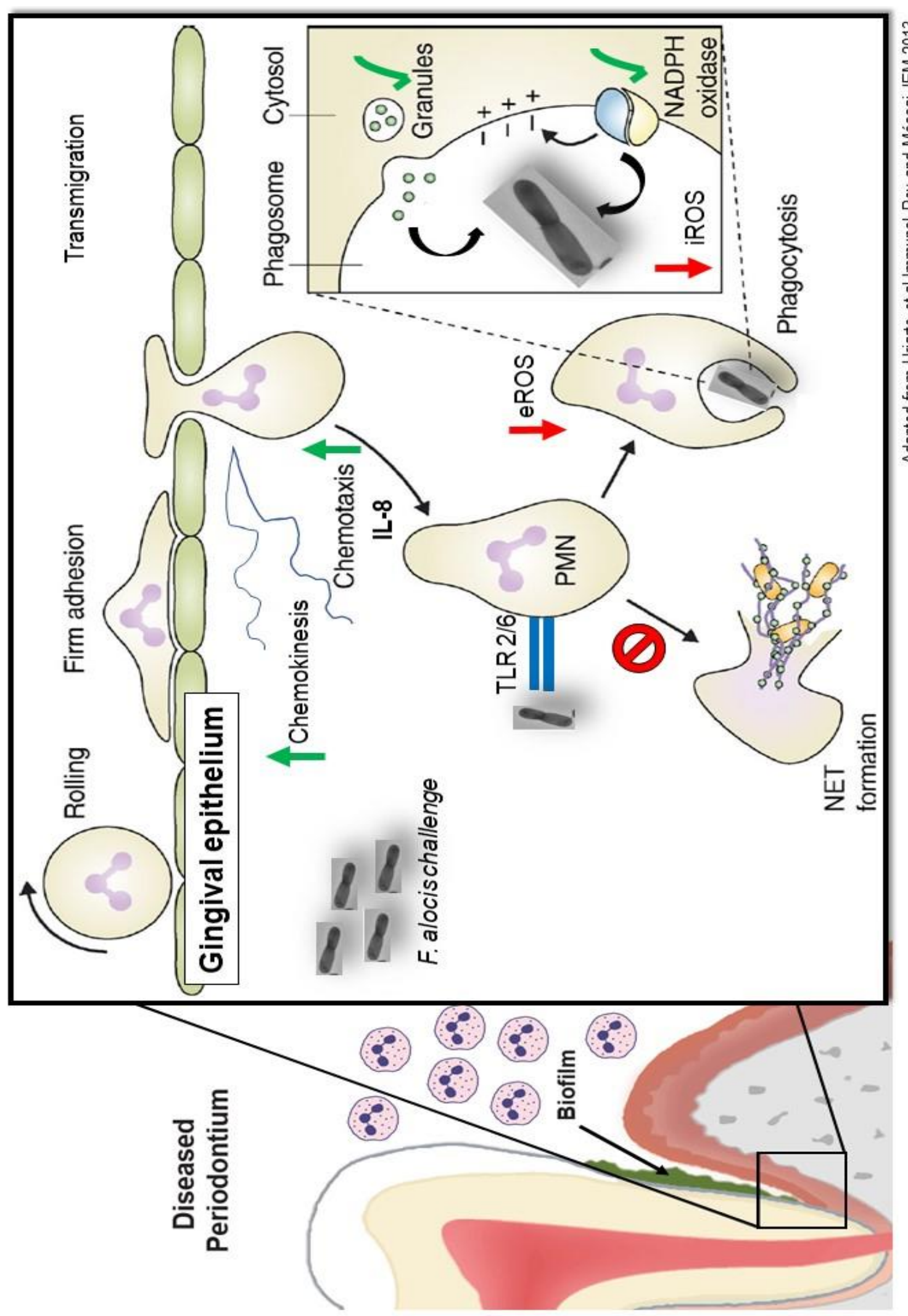

Figure 6-1. The effects of $F$. alocis challenge on human neutrophil effector mechanisms. 


\section{REFERENCES}

1. Darveau RP. 2010. Periodontitis: a polymicrobial disruption of host homeostasis. Nat Rev Microbiol 8:481-90.

2. Aruni W, Chioma O, Fletcher HM. 2014. Filifactor alocis: The Newly Discovered Kid on the Block with Special Talents. J Dent Res 93:725-32.

3. Bosshardt DD, Lang NP. 2005. The junctional epithelium: from health to disease. J Dent Res 84:9-20.

4. Duerkop BA, Vaishnava S, Hooper LV. 2009. Immune responses to the microbiota at the intestinal mucosal surface. Immunity 31:368-76.

5. Berezow AB, Darveau RP. 2011. Microbial shift and periodontitis. Periodontol 2000 55:36-47.

6. Champagne CM, Buchanan W, Reddy MS, Preisser JS, Beck JD, Offenbacher S. 2003. Potential for gingival crevice fluid measures as predictors of risk for periodontal diseases. Periodontol 2000 31:167-80.

7. Page RC, Kornman KS. 1997. The pathogenesis of human periodontitis: an introduction. Periodontol 2000 14:9-11.

8. Van Dyke TE. 2008. The management of inflammation in periodontal disease. J Periodontol 79:1601-8.

9. Oliver RC, Brown LJ, Loe H. 1991. Variations in the prevalence and extent of periodontitis. J Am Dent Assoc 122:43-8.

10. Eke PI, Dye BA, Wei L, Thornton-Evans GO, Genco RJ. 2012. Prevalence of periodontitis in adults in the United States: 2009 and 2010. J Dent Res 91:914-20.

11. Thornton-Evans G, Eke P, Wei L, Palmer A, Moeti R, Hutchins S, Borrell LN. 2013. Periodontitis among adults aged $>/=30$ years - United States, 2009-2010. MMWR Suppl 62:129-35.

12. Eke PI, Dye BA, Wei L, Slade GD, Thornton-Evans GO, Borgnakke WS, Taylor GW, Page RC, Beck JD, Genco RJ. 2015. Update on Prevalence of Periodontitis in Adults in the United States: NHANES 2009 to 2012. J Periodontol 86:611-22.

13. Haytac MC, Ozcelik O, Mariotti A. 2013. Periodontal disease in men. Periodontol 2000 61:252-65.

14. Dewhirst FE, Chen T, Izard J, Paster BJ, Tanner AC, Yu WH, Lakshmanan A, Wade WG. 2010. The human oral microbiome. J Bacteriol 192:5002-17.

15. Eke PI, Genco RJ. 2007. CDC Periodontal Disease Surveillance Project: background, objectives, and progress report. J Periodontol 78:1366-71.

16. Eke PI, Thornton-Evans G, Dye B, Genco R. 2012. Advances in surveillance of periodontitis: the Centers for Disease Control and Prevention periodontal disease surveillance project. J Periodontol 83:1337-42.

17. Socransky SS, Haffajee AD. 1994. Evidence of bacterial etiology: a historical perspective. Periodontol 2000 5:7-25. 
18. Socransky SS, Haffajee AD, Cugini MA, Smith C, Kent RL, Jr. 1998. Microbial complexes in subgingival plaque. J Clin Periodontol 25:134-44.

19. Ximenez-Fyvie LA, Haffajee AD, Socransky SS. 2000. Comparison of the microbiota of supra- and subgingival plaque in health and periodontitis. J Clin Periodontol 27:648-57.

20. Darveau RP, Tanner A, Page RC. 1997. The microbial challenge in periodontitis. Periodontol 2000 14:12-32.

21. Tanner A, Kent R, Maiden MF, Taubman MA. 1996. Clinical, microbiological and immunological profile of healthy, gingivitis and putative active periodontal subjects. $\mathrm{J}$ Periodontal Res 31:195-204.

22. Huyghe A, Francois P, Charbonnier Y, Tangomo-Bento M, Bonetti EJ, Paster BJ, Bolivar I, Baratti-Mayer D, Pittet D, Schrenzel J. 2008. Novel microarray design strategy to study complex bacterial communities. Appl Environ Microbiol 74:1876-85.

23. Kroes I, Lepp PW, Relman DA. 1999. Bacterial diversity within the human subgingival crevice. Proc Natl Acad Sci U S A 96:14547-52.

24. Berglundh T, Liljenberg B, Lindhe J. 1999. Some effects of periodontal therapy on local and systemic immunological parameters. J Clin Periodontol 26:91-8.

25. Yoshinari N, Kawase H, Mitani A, Ito M, Sugiishi S, Matsuoka M, Shirozu N, Ishihara Y, Bito B, Hiraga M, Arakawa K, Noguchi T. 2004. Effects of scaling and root planing on the amounts of interleukin-1 and interleukin-1 receptor antagonist and the mRNA expression of interleukin-1beta in gingival crevicular fluid and gingival tissues. J Periodontal Res 39:158-67.

26. Roberts FA, Hockett RD, Jr., Bucy RP, Michalek SM. 1997. Quantitative assessment of inflammatory cytokine gene expression in chronic adult periodontitis. Oral Microbiol Immunol 12:336-44.

27. Giannobile WV. 1997. Crevicular fluid biomarkers of oral bone loss. Curr Opin Periodontol 4:11-20.

28. Kamma J, Mombelli A, Tsinidou K, Vasdekis V, Giannopoulou C. 2009. Cytokines in gingival crevicular fluid of adolescents and young adults. Oral Microbiol Immunol 24:710 .

29. Thunell DH, Tymkiw KD, Johnson GK, Joly S, Burnell KK, Cavanaugh JE, Brogden KA, Guthmiller JM. 2010. A multiplex immunoassay demonstrates reductions in gingival crevicular fluid cytokines following initial periodontal therapy. J Periodontal Res 45:14852.

30. Brown MR, Allison DG, Gilbert P. 1988. Resistance of bacterial biofilms to antibiotics: a growth-rate related effect? J Antimicrob Chemother 22:777-80.

31. Hall-Stoodley L, Stoodley P. 2009. Evolving concepts in biofilm infections. Cell Microbiol 11:1034-43.

32. Fux CA, Costerton JW, Stewart PS, Stoodley P. 2005. Survival strategies of infectious biofilms. Trends Microbiol 13:34-40.

33. Warinner C, Rodrigues JF, Vyas R, Trachsel C, Shved N, Grossmann J, Radini A, Hancock Y, Tito RY, Fiddyment S, Speller C, Hendy J, Charlton S, Luder HU, SalazarGarcia DC, Eppler E, Seiler R, Hansen LH, Castruita JA, Barkow-Oesterreicher S, Teoh KY, Kelstrup CD, Olsen JV, Nanni P, Kawai T, Willerslev E, von Mering C, Lewis CM, Jr., Collins MJ, Gilbert MT, Ruhli F, Cappellini E. 2014. Pathogens and host immunity in the ancient human oral cavity. Nat Genet 46:336-44.

34. Marsh PD. 2003. Are dental diseases examples of ecological catastrophes? Microbiology 149:279-94.

35. Pihlstrom BL, Michalowicz BS, Johnson NW. 2005. Periodontal diseases. Lancet 366:1809-20. 
36. Coats SR, Pham TT, Bainbridge BW, Reife RA, Darveau RP. 2005. MD-2 mediates the ability of tetra-acylated and penta-acylated lipopolysaccharides to antagonize Escherichia coli lipopolysaccharide at the TLR4 signaling complex. J Immunol 175:4490-8.

37. Coats SR, Do CT, Karimi-Naser LM, Braham PH, Darveau RP. 2007. Antagonistic lipopolysaccharides block E. coli lipopolysaccharide function at human TLR4 via interaction with the human MD-2 lipopolysaccharide binding site. Cell Microbiol 9:1191202.

38. Socransky SS, Haffajee AD. 2005. Periodontal microbial ecology. Periodontol 2000 38:135-87.

39. Frank DN, St Amand AL, Feldman RA, Boedeker EC, Harpaz N, Pace NR. 2007. Molecular-phylogenetic characterization of microbial community imbalances in human inflammatory bowel diseases. Proc Natl Acad Sci U S A 104:13780-5.

40. Usviatsov B, Parshuta LI, Dolgov VA. 2000. [Microbial biocenosis in the mucous membranes of the nose and the middle ear in patients with purulent otitis]. Zh Mikrobiol Epidemiol Immunobiol:85-8.

41. Oakley BB, Fiedler TL, Marrazzo JM, Fredricks DN. 2008. Diversity of human vaginal bacterial communities and associations with clinically defined bacterial vaginosis. Appl Environ Microbiol 74:4898-909.

42. Darveau RP. 2009. The oral microbial consortium's interaction with the periodontal innate defense system. DNA Cell Biol 28:389-95.

43. Aas JA, Paster BJ, Stokes LN, Olsen I, Dewhirst FE. 2005. Defining the normal bacterial flora of the oral cavity. J Clin Microbiol 43:5721-32.

44. Moffatt CE, Whitmore SE, Griffen AL, Leys EJ, Lamont RJ. 2011. Filifactor alocis interactions with gingival epithelial cells. Mol Oral Microbiol 26:365-73.

45. Lamont RJ, Jenkinson HF. 1998. Life below the gum line: pathogenic mechanisms of Porphyromonas gingivalis. Microbiol Mol Biol Rev 62:1244-63.

46. Holt SC, Ebersole JL. 2005. Porphyromonas gingivalis, Treponema denticola, and Tannerella forsythia: the "red complex", a prototype polybacterial pathogenic consortium in periodontitis. Periodontol 2000 38:72-122.

47. Feng Z, Weinberg A. 2006. Role of bacteria in health and disease of periodontal tissues. Periodontol 2000 40:50-76.

48. Sharma A. 2010. Virulence mechanisms of Tannerella forsythia. Periodontol 2000 54:106-16.

49. Frederick JR, Sarkar J, McDowell JV, Marconi RT. 2011. Molecular signaling mechanisms of the periopathogen, Treponema denticola. J Dent Res 90:1155-63.

50. Kumar PS, Leys EJ, Bryk JM, Martinez FJ, Moeschberger ML, Griffen AL. 2006. Changes in periodontal health status are associated with bacterial community shifts as assessed by quantitative 16S cloning and sequencing. J Clin Microbiol 44:3665-73.

51. Griffen AL, Beall CJ, Firestone ND, Gross EL, Difranco JM, Hardman JH, Vriesendorp B, Faust RA, Janies DA, Leys EJ. 2011. CORE: a phylogenetically-curated 16S rDNA database of the core oral microbiome. PLoS One 6:e19051.

52. Wade WG. 2011. Has the use of molecular methods for the characterization of the human oral microbiome changed our understanding of the role of bacteria in the pathogenesis of periodontal disease? J Clin Periodontol 38 Suppl 11:7-16.

53. Paster BJ, Boches SK, Galvin JL, Ericson RE, Lau CN, Levanos VA, Sahasrabudhe A, Dewhirst FE. 2001. Bacterial diversity in human subgingival plaque. J Bacteriol 183:3770-83.

54. Dahlen G, Leonhardt A. 2006. A new checkerboard panel for testing bacterial markers in periodontal disease. Oral Microbiol Immunol 21:6-11.

55. Jalava J, Eerola E. 1999. Phylogenetic analysis of Fusobacterium alocis and Fusobacterium sulci based on 16S rRNA gene sequences: proposal of Filifactor alocis 
(Cato, Moore and Moore) comb. nov. and Eubacterium sulci (Cato, Moore and Moore) comb. nov. Int J Syst Bacteriol 49 Pt 4:1375-9.

56. Downes J, Munson MA, Spratt DA, Kononen E, Tarkka E, Jousimies-Somer H, Wade WG. 2001. Characterisation of Eubacterium-like strains isolated from oral infections. J Med Microbiol 50:947-51.

57. Colombo AP, Boches SK, Cotton SL, Goodson JM, Kent R, Haffajee AD, Socransky SS, Hasturk H, Van Dyke TE, Dewhirst F, Paster BJ. 2009. Comparisons of subgingival microbial profiles of refractory periodontitis, severe periodontitis, and periodontal health using the human oral microbe identification microarray. J Periodontol 80:1421-32.

58. Aruni AW, Zhang K, Dou Y, Fletcher H. 2014. Proteome analysis of coinfection of epithelial cells with Filifactor alocis and Porphyromonas gingivalis shows modulation of pathogen and host regulatory pathways. Infect Immun 82:3261-74.

59. Kumar PS, Griffen AL, Barton JA, Paster BJ, Moeschberger ML, Leys EJ. 2003. New bacterial species associated with chronic periodontitis. J Dent Res 82:338-44.

60. Aruni AW, Roy F, Fletcher HM. 2011. Filifactor alocis has virulence attributes that can enhance its persistence under oxidative stress conditions and mediate invasion of epithelial cells by porphyromonas gingivalis. Infect Immun 79:3872-86.

61. Wang Q, Jotwani R, Le J, Krauss JL, Potempa J, Coventry SC, Uriarte SM, Lamont RJ. 2014. Filifactor alocis infection and inflammatory responses in the mouse subcutaneous chamber model. Infect Immun 82:1205-12.

62. Uematsu H, Sato N, Hossain MZ, Ikeda T, Hoshino E. 2003. Degradation of arginine and other amino acids by butyrate-producing asaccharolytic anaerobic Gram-positive rods in periodontal pockets. Arch Oral Biol 48:423-9.

63. Wang Q, Wright CJ, Dingming H, Uriarte SM, Lamont RJ. 2013. Oral community interactions of Filifactor alocis in vitro. PLoS One 8:e76271.

64. Aruni AW, Roy F, Sandberg L, Fletcher HM. 2012. Proteome variation among Filifactor alocis strains. Proteomics 12:3343-64.

65. Foulquier C, Sebbag M, Clavel C, Chapuy-Regaud S, Al Badine R, Mechin MC, Vincent C, Nachat R, Yamada M, Takahara H, Simon M, Guerrin M, Serre G. 2007. Peptidyl arginine deiminase type 2 (PAD-2) and PAD-4 but not PAD-1, PAD-3, and PAD-6 are expressed in rheumatoid arthritis synovium in close association with tissue inflammation. Arthritis Rheum 56:3541-53.

66. Preshaw PM, Taylor JJ. 2011. How has research into cytokine interactions and their role in driving immune responses impacted our understanding of periodontitis? J Clin Periodontol 38 Suppl 11:60-84.

67. Birkedal-Hansen H. 1993. Role of cytokines and inflammatory mediators in tissue destruction. J Periodontal Res 28:500-10.

68. Graves D. 2008. Cytokines that promote periodontal tissue destruction. J Periodontol 79:1585-91.

69. Graves DT, Cochran D. 2003. The contribution of interleukin-1 and tumor necrosis factor to periodontal tissue destruction. J Periodontol 74:391-401.

70. Schlafer S, Riep B, Griffen AL, Petrich A, Hubner J, Berning M, Friedmann A, Gobel UB, Moter A. 2010. Filifactor alocis--involvement in periodontal biofilms. BMC Microbiol 10:66.

71. Pollanen MT, Paino A, Ihalin R. 2013. Environmental stimuli shape biofilm formation and the virulence of periodontal pathogens. Int J Mol Sci 14:17221-37.

72. Amulic B, Cazalet C, Hayes GL, Metzler KD, Zychlinsky A. 2012. Neutrophil function: from mechanisms to disease. Annu Rev Immunol 30:459-89.

73. Bardoel BW, Kenny EF, Sollberger G, Zychlinsky A. 2014. The balancing act of neutrophils. Cell Host Microbe 15:526-36.

74. Borregaard N. 2010. Neutrophils, from marrow to microbes. Immunity 33:657-70. 
75. Mantovani A, Cassatella MA, Costantini C, Jaillon S. 2011. Neutrophils in the activation and regulation of innate and adaptive immunity. Nat Rev Immunol 11:519-31.

76. Colotta F, Re F, Polentarutti N, Sozzani S, Mantovani A. 1992. Modulation of granulocyte survival and programmed cell death by cytokines and bacterial products. Blood 80:2012-20.

77. Nathan C. 2006. Neutrophils and immunity: challenges and opportunities. Nat Rev Immunol 6:173-82.

78. Segal AW. 2005. How neutrophils kill microbes. Annu Rev Immunol 23:197-223.

79. Bugl S, Wirths S, Muller MR, Radsak MP, Kopp HG. 2012. Current insights into neutrophil homeostasis. Ann N Y Acad Sci 1266:171-8.

80. Sabroe I, Dower SK, Whyte MK. 2005. The role of Toll-like receptors in the regulation of neutrophil migration, activation, and apoptosis. Clin Infect Dis 41 Suppl 7:S421-6.

81. Hayashi F, Means TK, Luster AD. 2003. Toll-like receptors stimulate human neutrophil function. Blood 102:2660-9.

82. Kurt-Jones EA, Mandell L, Whitney C, Padgett A, Gosselin K, Newburger PE, Finberg RW. 2002. Role of toll-like receptor 2 (TLR2) in neutrophil activation: GM-CSF enhances TLR2 expression and TLR2-mediated interleukin 8 responses in neutrophils. Blood 100:1860-8.

83. Sabroe I, Jones EC, Usher LR, Whyte MK, Dower SK. 2002. Toll-like receptor (TLR)2 and TLR4 in human peripheral blood granulocytes: a critical role for monocytes in leukocyte lipopolysaccharide responses. J Immunol 168:4701-10.

84. Mocsai A. 2013. Diverse novel functions of neutrophils in immunity, inflammation, and beyond. J Exp Med 210:1283-99.

85. Liu F, Wu HY, Wesselschmidt R, Kornaga T, Link DC. 1996. Impaired production and increased apoptosis of neutrophils in granulocyte colony-stimulating factor receptordeficient mice. Immunity 5:491-501.

86. Karsunky H, Zeng H, Schmidt T, Zevnik B, Kluge R, Schmid KW, Duhrsen U, Moroy T. 2002. Inflammatory reactions and severe neutropenia in mice lacking the transcriptional repressor Gfi1. Nat Genet 30:295-300.

87. Hock H, Hamblen MJ, Rooke HM, Traver D, Bronson RT, Cameron S, Orkin SH. 2003. Intrinsic requirement for zinc finger transcription factor Gfi-1 in neutrophil differentiation. Immunity 18:109-20.

88. Jonsson H, Allen P, Peng SL. 2005. Inflammatory arthritis requires Foxo3a to prevent Fas ligand-induced neutrophil apoptosis. Nat Med 11:666-71.

89. Dzhagalov I, St John A, He YW. 2007. The antiapoptotic protein Mcl-1 is essential for the survival of neutrophils but not macrophages. Blood 109:1620-6.

90. Ordonez-Rueda D, Jonsson F, Mancardi DA, Zhao W, Malzac A, Liang Y, Bertosio E, Grenot P, Blanquet V, Sabrautzki S, de Angelis MH, Meresse S, Duprez E, Bruhns P, Malissen B, Malissen M. 2012. A hypomorphic mutation in the Gfi1 transcriptional repressor results in a novel form of neutropenia. Eur J Immunol 42:2395-408.

91. Daley JM, Thomay AA, Connolly MD, Reichner JS, Albina JE. 2008. Use of Ly6Gspecific monoclonal antibody to deplete neutrophils in mice. J Leukoc Biol 83:64-70.

92. Abram CL, Roberge GL, Pao LI, Neel BG, Lowell CA. 2013. Distinct roles for neutrophils and dendritic cells in inflammation and autoimmunity in motheaten mice. Immunity 38:489-501.

93. Bruhns P. 2012. Properties of mouse and human IgG receptors and their contribution to disease models. Blood 119:5640-9.

94. Davey MS, Tamassia N, Rossato M, Bazzoni F, Calzetti F, Bruderek K, Sironi M, Zimmer L, Bottazzi B, Mantovani A, Brandau S, Moser B, Eberl M, Cassatella MA. 2011. Failure to detect production of IL-10 by activated human neutrophils. Nat Immunol 12:1017-8; author reply 1018-20. 
95. Mestas J, Hughes CC. 2004. Of mice and not men: differences between mouse and human immunology. J Immunol 172:2731-8.

96. Seok J, Warren HS, Cuenca AG, Mindrinos MN, Baker HV, Xu W, Richards DR, McDonald-Smith GP, Gao H, Hennessy L, Finnerty CC, Lopez CM, Honari S, Moore EE, Minei JP, Cuschieri J, Bankey PE, Johnson JL, Sperry J, Nathens AB, Billiar TR, West MA, Jeschke MG, Klein MB, Gamelli RL, Gibran NS, Brownstein BH, MillerGraziano C, Calvano SE, Mason PH, Cobb JP, Rahme LG, Lowry SF, Maier RV, Moldawer LL, Herndon DN, Davis RW, Xiao W, Tompkins RG. 2013. Genomic responses in mouse models poorly mimic human inflammatory diseases. Proc Natl Acad Sci U S A 110:3507-12.

97. Bruehl RE, Moore KL, Lorant DE, Borregaard N, Zimmerman GA, McEver RP, Bainton DF. 1997. Leukocyte activation induces surface redistribution of P-selectin glycoprotein ligand-1. J Leukoc Biol 61:489-99.

98. Buscher K, Riese SB, Shakibaei M, Reich C, Dernedde J, Tauber R, Ley K. 2010. The transmembrane domains of L-selectin and CD44 regulate receptor cell surface positioning and leukocyte adhesion under flow. J Biol Chem 285:13490-7.

99. Steegmaier M, Borges E, Berger J, Schwarz H, Vestweber D. 1997. The E-selectin-ligand ESL-1 is located in the Golgi as well as on microvilli on the cell surface. J Cell Sci 110 ( Pt 6):687-94.

100. Hidalgo A, Peired AJ, Wild MK, Vestweber D, Frenette PS. 2007. Complete identification of E-selectin ligands on neutrophils reveals distinct functions of PSGL-1, ESL-1, and CD44. Immunity 26:477-89.

101. Phillipson M, Kaur J, Colarusso P, Ballantyne CM, Kubes P. 2008. Endothelial domes encapsulate adherent neutrophils and minimize increases in vascular permeability in paracellular and transcellular emigration. PLoS One 3:e1649.

102. Kang T, Yi J, Guo A, Wang X, Overall CM, Jiang W, Elde R, Borregaard N, Pei D. 2001. Subcellular distribution and cytokine- and chemokine-regulated secretion of leukolysin/MT6-MMP/MMP-25 in neutrophils. J Biol Chem 276:21960-8.

103. Nauseef WM, Borregaard N. 2014. Neutrophils at work. Nat Immunol 15:602-11.

104. Scapini P, Lapinet-Vera JA, Gasperini S, Calzetti F, Bazzoni F, Cassatella MA. 2000. The neutrophil as a cellular source of chemokines. Immunol Rev 177:195-203.

105. Scapini P, Carletto A, Nardelli B, Calzetti F, Roschke V, Merigo F, Tamassia N, Pieropan S, Biasi D, Sbarbati A, Sozzani S, Bambara L, Cassatella MA. 2005. Proinflammatory mediators elicit secretion of the intracellular B-lymphocyte stimulator pool (BLyS) that is stored in activated neutrophils: implications for inflammatory diseases. Blood 105:830-7.

106. Theilgaard-Monch K, Knudsen S, Follin P, Borregaard N. 2004. The transcriptional activation program of human neutrophils in skin lesions supports their important role in wound healing. J Immunol 172:7684-93.

107. Alvarez Y, Tang X, Coligan JE, Borrego F. 2008. The CD300a (IRp60) inhibitory receptor is rapidly up-regulated on human neutrophils in response to inflammatory stimuli and modulates CD32a (FcgammaRIIa) mediated signaling. Mol Immunol 45:2538.

108. Daeron M, Jaeger S, Du Pasquier L, Vivier E. 2008. Immunoreceptor tyrosine-based inhibition motifs: a quest in the past and future. Immunol Rev 224:11-43.

109. Ducker TP, Skubitz KM. 1992. Subcellular localization of CD66, CD67, and NCA in human neutrophils. J Leukoc Biol 52:11-6.

110. Skubitz KM, Skubitz AP. 2008. Interdependency of CEACAM-1, -3, -6, and -8 induced human neutrophil adhesion to endothelial cells. J Transl Med 6:78. 
111. Steevels TA, Lebbink RJ, Westerlaken GH, Coffer PJ, Meyaard L. 2010. Signal inhibitory receptor on leukocytes-1 is a novel functional inhibitory immune receptor expressed on human phagocytes. J Immunol 184:4741-8.

112. Jonsson F, Mancardi DA, Albanesi M, Bruhns P. 2013. Neutrophils in local and systemic antibody-dependent inflammatory and anaphylactic reactions. J Leukoc Biol 94:643-56.

113. Anderson SI, Hotchin NA, Nash GB. 2000. Role of the cytoskeleton in rapid activation of $\mathrm{CD} 1 \mathrm{~b} / \mathrm{CD} 18$ function and its subsequent downregulation in neutrophils. J Cell Sci 113 ( Pt 15):2737-45.

114. Srinivasan S, Wang F, Glavas S, Ott A, Hofmann F, Aktories K, Kalman D, Bourne HR. 2003. Rac and $\mathrm{Cdc} 42$ play distinct roles in regulating $\mathrm{PI}(3,4,5) \mathrm{P} 3$ and polarity during neutrophil chemotaxis. J Cell Biol 160:375-85.

115. Parent CA. 2004. Making all the right moves: chemotaxis in neutrophils and Dictyostelium. Curr Opin Cell Biol 16:4-13.

116. Capoccia BJ, Gregory AD, Link DC. 2008. Recruitment of the inflammatory subset of monocytes to sites of ischemia induces angiogenesis in a monocyte chemoattractant protein-1-dependent fashion. J Leukoc Biol 84:760-8.

117. Yin X, Knecht DA, Lynes MA. 2005. Metallothionein mediates leukocyte chemotaxis. BMC Immunol 6:21.

118. Selvatici R, Falzarano S, Mollica A, Spisani S. 2006. Signal transduction pathways triggered by selective formylpeptide analogues in human neutrophils. Eur J Pharmacol 534:1-11.

119. Wong CH, Heit B, Kubes P. 2010. Molecular regulators of leucocyte chemotaxis during inflammation. Cardiovasc Res 86:183-91.

120. Gambardella L, Vermeren S. 2013. Molecular players in neutrophil chemotaxis--focus on PI3K and small GTPases. J Leukoc Biol 94:603-12.

121. Lacalle RA, Peregil RM, Albar JP, Merino E, Martinez AC, Merida I, Manes S. 2007. Type I phosphatidylinositol 4-phosphate 5-kinase controls neutrophil polarity and directional movement. J Cell Biol 179:1539-53.

122. Heit B, Liu L, Colarusso P, Puri KD, Kubes P. 2008. PI3K accelerates, but is not required for, neutrophil chemotaxis to fMLP. J Cell Sci 121:205-14.

123. Heit B, Tavener S, Raharjo E, Kubes P. 2002. An intracellular signaling hierarchy determines direction of migration in opposing chemotactic gradients. J Cell Biol 159:91102.

124. Heit B, Colarusso P, Kubes P. 2005. Fundamentally different roles for LFA-1, Mac-1 and alpha4-integrin in neutrophil chemotaxis. J Cell Sci 118:5205-20.

125. Murdoch C, Finn A. 2000. Chemokine receptors and their role in inflammation and infectious diseases. Blood 95:3032-43.

126. Luong TT, Lee CY. 2002. Overproduction of type 8 capsular polysaccharide augments Staphylococcus aureus virulence. Infect Immun 70:3389-95.

127. Allen LA, Beecher BR, Lynch JT, Rohner OV, Wittine LM. 2005. Helicobacter pylori disrupts NADPH oxidase targeting in human neutrophils to induce extracellular superoxide release. J Immunol 174:3658-67.

128. McCaffrey RL, Schwartz JT, Lindemann SR, Moreland JG, Buchan BW, Jones BD, Allen LA. 2010. Multiple mechanisms of NADPH oxidase inhibition by type A and type B Francisella tularensis. J Leukoc Biol 88:791-805.

129. Joiner KA, Ganz T, Albert J, Rotrosen D. 1989. The opsonizing ligand on Salmonella typhimurium influences incorporation of specific, but not azurophil, granule constituents into neutrophil phagosomes. J Cell Biol 109:2771-82.

130. Staali L, Bauer S, Morgelin M, Bjorck L, Tapper H. 2006. Streptococcus pyogenes bacteria modulate membrane traffic in human neutrophils and selectively inhibit azurophilic granule fusion with phagosomes. Cell Microbiol 8:690-703. 
131. Brogden KA. 2005. Antimicrobial peptides: pore formers or metabolic inhibitors in bacteria? Nat Rev Microbiol 3:238-50.

132. Smirnov A, Daily KP, Criss AK. 2014. Assembly of NADPH oxidase in human neutrophils is modulated by the opacity-associated protein expression State of Neisseria gonorrhoeae. Infect Immun 82:1036-44.

133. Nauseef WM. 2004. Assembly of the phagocyte NADPH oxidase. Histochem Cell Biol 122:277-91.

134. Ueyama T, Kusakabe T, Karasawa S, Kawasaki T, Shimizu A, Son J, Leto TL, Miyawaki A, Saito N. 2008. Sequential binding of cytosolic Phox complex to phagosomes through regulated adaptor proteins: evaluation using the novel monomeric Kusabira-Green System and live imaging of phagocytosis. J Immunol 181:629-40.

135. El-Benna J, Dang PM, Gougerot-Pocidalo MA, Marie JC, Braut-Boucher F. 2009. p47phox, the phagocyte NADPH oxidase/NOX2 organizer: structure, phosphorylation and implication in diseases. Exp Mol Med 41:217-25.

136. Kim C, Dinauer MC. 2001. Rac2 is an essential regulator of neutrophil nicotinamide adenine dinucleotide phosphate oxidase activation in response to specific signaling pathways. J Immunol 166:1223-32.

137. Koga H, Terasawa H, Nunoi H, Takeshige K, Inagaki F, Sumimoto H. 1999. Tetratricopeptide repeat (TPR) motifs of $\mathrm{p} 67$ (phox) participate in interaction with the small GTPase Rac and activation of the phagocyte NADPH oxidase. J Biol Chem 274:25051-60.

138. Carlyon JA, Ryan D, Archer K, Fikrig E. 2005. Effects of Anaplasma phagocytophilum on host cell ferritin mRNA and protein levels. Infect Immun 73:7629-36.

139. McCaffrey RL, Allen LA. 2006. Francisella tularensis LVS evades killing by human neutrophils via inhibition of the respiratory burst and phagosome escape. J Leukoc Biol 80:1224-30.

140. JW IJ, Mueller AC. 2004. Neutrophil NADPH oxidase is reduced at the Anaplasma phagocytophilum phagosome. Infect Immun 72:5392-401.

141. Kuhns DB, Alvord WG, Heller T, Feld JJ, Pike KM, Marciano BE, Uzel G, DeRavin SS, Priel DA, Soule BP, Zarember KA, Malech HL, Holland SM, Gallin JI. 2010. Residual NADPH oxidase and survival in chronic granulomatous disease. N Engl J Med 363:260010 .

142. Matute JD, Arias AA, Wright NA, Wrobel I, Waterhouse CC, Li XJ, Marchal CC, Stull ND, Lewis DB, Steele M, Kellner JD, Yu W, Meroueh SO, Nauseef WM, Dinauer MC. 2009. A new genetic subgroup of chronic granulomatous disease with autosomal recessive mutations in p40 phox and selective defects in neutrophil NADPH oxidase activity. Blood 114:3309-15.

143. Allen LA, McCaffrey RL. 2007. To activate or not to activate: distinct strategies used by Helicobacter pylori and Francisella tularensis to modulate the NADPH oxidase and survive in human neutrophils. Immunol Rev 219:103-17.

144. Nordenfelt P, Tapper H. 2011. Phagosome dynamics during phagocytosis by neutrophils. J Leukoc Biol 90:271-84.

145. Decoursey TE, Ligeti E. 2005. Regulation and termination of NADPH oxidase activity. Cell Mol Life Sci 62:2173-93.

146. Uriarte SM, Rane MJ, Luerman GC, Barati MT, Ward RA, Nauseef WM, McLeish KR. 2011. Granule exocytosis contributes to priming and activation of the human neutrophil respiratory burst. J Immunol 187:391-400.

147. Hajishengallis G. 2015. Periodontitis: from microbial immune subversion to systemic inflammation. Nat Rev Immunol 15:30-44.

148. Brinkmann V, Reichard U, Goosmann C, Fauler B, Uhlemann Y, Weiss DS, Weinrauch Y, Zychlinsky A. 2004. Neutrophil extracellular traps kill bacteria. Science 303:1532-5. 
149. Cooper PR, Palmer LJ, Chapple IL. 2013. Neutrophil extracellular traps as a new paradigm in innate immunity: friend or foe? Periodontol 2000 63:165-97.

150. Kraemer BF, Campbell RA, Schwertz H, Cody MJ, Franks Z, Tolley ND, Kahr WH, Lindemann S, Seizer P, Yost CC, Zimmerman GA, Weyrich AS. 2011. Novel antibacterial activities of beta-defensin 1 in human platelets: suppression of pathogen growth and signaling of neutrophil extracellular trap formation. PLoS Pathog 7:e1002355.

151. Beiter K, Wartha F, Albiger B, Normark S, Zychlinsky A, Henriques-Normark B. 2006. An endonuclease allows Streptococcus pneumoniae to escape from neutrophil extracellular traps. Curr Biol 16:401-7.

152. Greenlee-Wacker MC, Rigby KM, Kobayashi SD, Porter AR, DeLeo FR, Nauseef WM. 2014. Phagocytosis of Staphylococcus aureus by human neutrophils prevents macrophage efferocytosis and induces programmed necrosis. J Immunol 192:4709-17.

153. Miyake Y, Yamasaki S. 2012. Sensing necrotic cells. Adv Exp Med Biol 738:144-52.

154. Yipp BG, Petri B, Salina D, Jenne CN, Scott BN, Zbytnuik LD, Pittman K, Asaduzzaman M, Wu K, Meijndert HC, Malawista SE, de Boisfleury Chevance A, Zhang K, Conly J, Kubes P. 2012. Infection-induced NETosis is a dynamic process involving neutrophil multitasking in vivo. Nat Med 18:1386-93.

155. Yipp BG, Kubes P. 2013. NETosis: how vital is it? Blood 122:2784-94.

156. Chen K, Nishi H, Travers R, Tsuboi N, Martinod K, Wagner DD, Stan R, Croce K, Mayadas TN. 2012. Endocytosis of soluble immune complexes leads to their clearance by FcgammaRIIIB but induces neutrophil extracellular traps via FcgammaRIIA in vivo. Blood 120:4421-31.

157. Parker H, Dragunow M, Hampton MB, Kettle AJ, Winterbourn CC. 2012. Requirements for NADPH oxidase and myeloperoxidase in neutrophil extracellular trap formation differ depending on the stimulus. J Leukoc Biol 92:841-9.

158. Keshari RS, Jyoti A, Kumar S, Dubey M, Verma A, Srinag BS, Krishnamurthy H, Barthwal MK, Dikshit M. 2012. Neutrophil extracellular traps contain mitochondrial as well as nuclear DNA and exhibit inflammatory potential. Cytometry A 81:238-47.

159. Lande R, Ganguly D, Facchinetti V, Frasca L, Conrad C, Gregorio J, Meller S, Chamilos G, Sebasigari R, Riccieri V, Bassett R, Amuro H, Fukuhara S, Ito T, Liu YJ, Gilliet M. 2011. Neutrophils activate plasmacytoid dendritic cells by releasing self-DNA-peptide complexes in systemic lupus erythematosus. Sci Transl Med 3:73ra19.

160. Thomas GM, Carbo C, Curtis BR, Martinod K, Mazo IB, Schatzberg D, Cifuni SM, Fuchs TA, von Andrian UH, Hartwig JH, Aster RH, Wagner DD. 2012. Extracellular DNA traps are associated with the pathogenesis of TRALI in humans and mice. Blood 119:6335-43.

161. Kessenbrock K, Krumbholz M, Schonermarck U, Back W, Gross WL, Werb Z, Grone HJ, Brinkmann V, Jenne DE. 2009. Netting neutrophils in autoimmune small-vessel vasculitis. Nat Med 15:623-5.

162. Caudrillier A, Kessenbrock K, Gilliss BM, Nguyen JX, Marques MB, Monestier M, Toy P, Werb Z, Looney MR. 2012. Platelets induce neutrophil extracellular traps in transfusion-related acute lung injury. J Clin Invest 122:2661-71.

163. Chow OA, von Kockritz-Blickwede M, Bright AT, Hensler ME, Zinkernagel AS, Cogen AL, Gallo RL, Monestier M, Wang Y, Glass CK, Nizet V. 2010. Statins enhance formation of phagocyte extracellular traps. Cell Host Microbe 8:445-54.

164. Massberg S, Grahl L, von Bruehl ML, Manukyan D, Pfeiler S, Goosmann C, Brinkmann V, Lorenz M, Bidzhekov K, Khandagale AB, Konrad I, Kennerknecht E, Reges K, Holdenrieder S, Braun S, Reinhardt C, Spannagl M, Preissner KT, Engelmann B. 2010. Reciprocal coupling of coagulation and innate immunity via neutrophil serine proteases. Nat Med 16:887-96. 
165. Barrientos L, Marin-Esteban V, de Chaisemartin L, Le-Moal VL, Sandre C, Bianchini E, Nicolas V, Pallardy M, Chollet-Martin S. 2013. An improved strategy to recover large fragments of functional human neutrophil extracellular traps. Front Immunol 4:166.

166. Zawrotniak M, Rapala-Kozik M. 2013. Neutrophil extracellular traps (NETs) - formation and implications. Acta Biochim Pol 60:277-84.

167. Yousefi S, Mihalache C, Kozlowski E, Schmid I, Simon HU. 2009. Viable neutrophils release mitochondrial DNA to form neutrophil extracellular traps. Cell Death Differ 16:1438-44.

168. Brinkmann V, Zychlinsky A. 2012. Neutrophil extracellular traps: is immunity the second function of chromatin? J Cell Biol 198:773-83.

169. Remijsen Q, Vanden Berghe T, Wirawan E, Asselbergh B, Parthoens E, De Rycke R, Noppen S, Delforge M, Willems J, Vandenabeele P. 2011. Neutrophil extracellular trap cell death requires both autophagy and superoxide generation. Cell Res 21:290-304.

170. Papayannopoulos V, Metzler KD, Hakkim A, Zychlinsky A. 2010. Neutrophil elastase and myeloperoxidase regulate the formation of neutrophil extracellular traps. J Cell Biol 191:677-91.

171. Berends ET, Horswill AR, Haste NM, Monestier M, Nizet V, von Kockritz-Blickwede M. 2010. Nuclease expression by Staphylococcus aureus facilitates escape from neutrophil extracellular traps. J Innate Immun 2:576-86.

172. Saffarzadeh M, Juenemann C, Queisser MA, Lochnit G, Barreto G, Galuska SP, Lohmeyer J, Preissner KT. 2012. Neutrophil extracellular traps directly induce epithelial and endothelial cell death: a predominant role of histones. PLoS One 7:e32366.

173. Rouault H, Mazouni K, Couturier L, Hakim V, Schweisguth F. 2010. Genome-wide identification of cis-regulatory motifs and modules underlying gene coregulation using statistics and phylogeny. Proc Natl Acad Sci U S A 107:14615-20.

174. Garcia-Romo GS, Caielli S, Vega B, Connolly J, Allantaz F, Xu Z, Punaro M, Baisch J, Guiducci C, Coffman RL, Barrat FJ, Banchereau J, Pascual V. 2011. Netting neutrophils are major inducers of type I IFN production in pediatric systemic lupus erythematosus. Sci Transl Med 3:73ra20.

175. Villanueva E, Yalavarthi S, Berthier CC, Hodgin JB, Khandpur R, Lin AM, Rubin CJ, Zhao W, Olsen SH, Klinker M, Shealy D, Denny MF, Plumas J, Chaperot L, Kretzler M, Bruce AT, Kaplan MJ. 2011. Netting neutrophils induce endothelial damage, infiltrate tissues, and expose immunostimulatory molecules in systemic lupus erythematosus. J Immunol 187:538-52.

176. Mayadas TN, Tsokos GC, Tsuboi N. 2009. Mechanisms of immune complex-mediated neutrophil recruitment and tissue injury. Circulation 120:2012-24.

177. Tonetti MS, Imboden MA, Lang NP. 1998. Neutrophil migration into the gingival sulcus is associated with transepithelial gradients of interleukin-8 and ICAM-1. J Periodontol 69:1139-47.

178. Schiott CR, Loe H. 1970. The origin and variation in number of leukocytes in the human saliva. J Periodontal Res 5:36-41.

179. Scott DA, Krauss J. 2012. Neutrophils in periodontal inflammation. Front Oral Biol 15:56-83.

180. Meng H, Xu L, Li Q, Han J, Zhao Y. 2007. Determinants of host susceptibility in aggressive periodontitis. Periodontol 2000 43:133-59.

181. Schenkein HA. 2006. Host responses in maintaining periodontal health and determining periodontal disease. Periodontol 2000 40:77-93.

182. Ryder MI. 2010. Comparison of neutrophil functions in aggressive and chronic periodontitis. Periodontol 2000 53:124-37.

183. Deas DE, Mackey SA, McDonnell HT. 2003. Systemic disease and periodontitis: manifestations of neutrophil dysfunction. Periodontol 2000 32:82-104. 
184. Uriarte SM, Edmisson JS, Jimenez-Flores E. 2016. Human neutrophils and oral microbiota: a constant tug-of-war between a harmonious and a discordant coexistence. Immunol Rev 273:282-98.

185. Nussbaum G, Shapira L. 2011. How has neutrophil research improved our understanding of periodontal pathogenesis? J Clin Periodontol 38 Suppl 11:49-59.

186. Hajishengallis E, Hajishengallis G. 2014. Neutrophil homeostasis and periodontal health in children and adults. J Dent Res 93:231-7.

187. Almeida RP, Vanet A, Witko-Sarsat V, Melchior M, McCabe D, Gabay JE. 1996. Azurocidin, a natural antibiotic from human neutrophils: expression, antimicrobial activity, and secretion. Protein Expr Purif 7:355-66.

188. Zaric S, Shelburne C, Darveau R, Quinn DJ, Weldon S, Taggart CC, Coulter WA. 2010. Impaired immune tolerance to Porphyromonas gingivalis lipopolysaccharide promotes neutrophil migration and decreased apoptosis. Infect Immun 78:4151-6.

189. Roberts FA, Darveau RP. 2015. Microbial protection and virulence in periodontal tissue as a function of polymicrobial communities: symbiosis and dysbiosis. Periodontol 2000 69:18-27.

190. El-Benna J, Hurtado-Nedelec M, Marzaioli V, Marie JC, Gougerot-Pocidalo MA, Dang PM. 2016. Priming of the neutrophil respiratory burst: role in host defense and inflammation. Immunol Rev 273:180-93.

191. Bender JS, Thang H, Glogauer M. 2006. Novel rinse assay for the quantification of oral neutrophils and the monitoring of chronic periodontal disease. J Periodontal Res 41:21420.

192. Liu RK, Cao CF, Meng HX, Gao Y. 2001. Polymorphonuclear neutrophils and their mediators in gingival tissues from generalized aggressive periodontitis. J Periodontol 72:1545-53.

193. Andersen E, Cimasoni G. 1993. A rapid and simple method for counting crevicular polymorphonuclear leucocytes. J Clin Periodontol 20:651-5.

194. Darveau RP, Belton CM, Reife RA, Lamont RJ. 1998. Local chemokine paralysis, a novel pathogenic mechanism for Porphyromonas gingivalis. Infect Immun 66:1660-5.

195. Bainbridge B, Verma RK, Eastman C, Yehia B, Rivera M, Moffatt C, Bhattacharyya I, Lamont RJ, Kesavalu L. 2010. Role of Porphyromonas gingivalis phosphoserine phosphatase enzyme SerB in inflammation, immune response, and induction of alveolar bone resorption in rats. Infect Immun 78:4560-9.

196. Lee WL, Grinstein S. 2004. Immunology. The tangled webs that neutrophils weave. Science 303:1477-8.

197. Vitkov L, Klappacher M, Hannig M, Krautgartner WD. 2009. Extracellular neutrophil traps in periodontitis. J Periodontal Res 44:664-72.

198. Hart TC, Atkinson JC. 2007. Mendelian forms of periodontitis. Periodontol 2000 45:95112.

199. Boxer L, Dale DC. 2002. Neutropenia: causes and consequences. Semin Hematol 39:7581.

200. Winkelstein JA, Marino MC, Johnston RB, Jr., Boyle J, Curnutte J, Gallin JI, Malech HL, Holland SM, Ochs H, Quie P, Buckley RH, Foster CB, Chanock SJ, Dickler H. 2000. Chronic granulomatous disease. Report on a national registry of 368 patients. Medicine (Baltimore) 79:155-69.

201. Picard C, von Bernuth H, Ghandil P, Chrabieh M, Levy O, Arkwright PD, McDonald D, Geha RS, Takada H, Krause JC, Creech CB, Ku CL, Ehl S, Marodi L, Al-Muhsen S, AlHajjar S, Al-Ghonaium A, Day-Good NK, Holland SM, Gallin JI, Chapel H, Speert DP, Rodriguez-Gallego C, Colino E, Garty BZ, Roifman C, Hara T, Yoshikawa H, Nonoyama S, Domachowske J, Issekutz AC, Tang M, Smart J, Zitnik SE, Hoarau C, Kumararatne DS, Thrasher AJ, Davies EG, Bethune C, Sirvent N, de Ricaud D, 
Camcioglu Y, Vasconcelos J, Guedes M, Vitor AB, Rodrigo C, Almazan F, Mendez M, Arostegui JI, Alsina L, et al. 2010. Clinical features and outcome of patients with IRAK4 and MyD88 deficiency. Medicine (Baltimore) 89:403-25.

202. Smeekens SP, van de Veerdonk FL, Kullberg BJ, Netea MG. 2013. Genetic susceptibility to Candida infections. EMBO Mol Med 5:805-13.

203. Hart TC, Shapira L, Van Dyke TE. 1994. Neutrophil defects as risk factors for periodontal diseases. J Periodontol 65:521-9.

204. Carrassi A, Abati S, Santarelli G, Vogel G. 1989. Periodontitis in a patient with chronic neutropenia. J Periodontol 60:352-7.

205. Page RC, Beatty P, Waldrop TC. 1987. Molecular basis for the functional abnormality in neutrophils from patients with generalized prepubertal periodontitis. J Periodontal Res 22:182-3.

206. Waldrop TC, Anderson DC, Hallmon WW, Schmalstieg FC, Jacobs RL. 1987.

Periodontal manifestations of the heritable Mac-1, LFA-1, deficiency syndrome. Clinical, histopathologic and molecular characteristics. J Periodontol 58:400-16.

207. Nagle DL, Karim MA, Woolf EA, Holmgren L, Bork P, Misumi DJ, McGrail SH, Dussault BJ, Jr., Perou CM, Boissy RE, Duyk GM, Spritz RA, Moore KJ. 1996.

Identification and mutation analysis of the complete gene for Chediak-Higashi syndrome. Nat Genet 14:307-11.

208. Delcourt-Debruyne EM, Boutigny HR, Hildebrand HF. 2000. Features of severe periodontal disease in a teenager with Chediak-Higashi syndrome. J Periodontol 71:81624.

209. Shibutani T, Gen K, Shibata M, Horiguchi Y, Kondo N, Iwayama Y. 2000. Long-term follow-up of periodontitis in a patient with Chediak-Higashi syndrome. A case report. $\mathrm{J}$ Periodontol 71:1024-8.

210. Nasidze I, Li J, Quinque D, Tang K, Stoneking M. 2009. Global diversity in the human salivary microbiome. Genome Res 19:636-43.

211. Avila M, Ojcius DM, Yilmaz O. 2009. The oral microbiota: living with a permanent guest. DNA Cell Biol 28:405-11.

212. Al-Ahmad A, Roth D, Wolkewitz M, Wiedmann-Al-Ahmad M, Follo M, Ratka-Kruger P, Deimling D, Hellwig E, Hannig C. 2010. Change in diet and oral hygiene over an 8week period: effects on oral health and oral biofilm. Clin Oral Investig 14:391-6.

213. Kang JG, Kim SH, Ahn TY. 2006. Bacterial diversity in the human saliva from different ages. J Microbiol 44:572-6.

214. Haffajee AD, Teles RP, Socransky SS. 2006. The effect of periodontal therapy on the composition of the subgingival microbiota. Periodontol 2000 42:219-58.

215. Teles RP, Haffajee AD, Socransky SS. 2006. Microbiological goals of periodontal therapy. Periodontol 2000 42:180-218.

216. Colombo AP, Haffajee AD, Dewhirst FE, Paster BJ, Smith CM, Cugini MA, Socransky SS. 1998. Clinical and microbiological features of refractory periodontitis subjects. J Clin Periodontol 25:169-80.

217. Colombo AP, Eftimiadi C, Haffajee AD, Cugini MA, Socransky SS. 1998. Serum IgG2 level, Gm(23) allotype and FcgammaRIIa and FcgammaRIIIb receptors in refractory periodontal disease. J Clin Periodontol 25:465-74.

218. Colombo AP, Sakellari D, Haffajee AD, Tanner A, Cugini MA, Socransky SS. 1998. Serum antibodies reacting with subgingival species in refractory periodontitis subjects. J Clin Periodontol 25:596-604.

219. Colombo AP, Haffajee AD, Smith CM, Cugini MA, Socransky SS. 1999. Discrimination of refractory periodontitis subjects using clinical and laboratory parameters alone and in combination. J Clin Periodontol 26:569-76. 
220. Bercy P, Lasserre J. 2007. Susceptibility to various oral antiseptics of Porphyromonas gingivalis W83 within a biofilm. Adv Ther 24:1181-91.

221. Bhatavadekar NB, Williams RC. 2009. New directions in host modulation for the management of periodontal disease. J Clin Periodontol 36:124-6.

222. Reddy MS, Geurs NC, Gunsolley JC. 2003. Periodontal host modulation with antiproteinase, anti-inflammatory, and bone-sparing agents. A systematic review. Ann Periodontol 8:12-37.

223. Konopka K, Goslinski T. 2007. Photodynamic therapy in dentistry. J Dent Res 86:694707.

224. Teughels W, Newman MG, Coucke W, Haffajee AD, Van Der Mei HC, Haake SK, Schepers E, Cassiman JJ, Van Eldere J, van Steenberghe D, Quirynen M. 2007. Guiding periodontal pocket recolonization: a proof of concept. J Dent Res 86:1078-82.

225. Booth V, Ashley FP, Lehner T. 1996. Passive immunization with monoclonal antibodies against Porphyromonas gingivalis in patients with periodontitis. Infect Immun 64:422-7.

226. Page RC, Lantz MS, Darveau R, Jeffcoat M, Mancl L, Houston L, Braham P, Persson GR. 2007. Immunization of Macaca fascicularis against experimental periodontitis using a vaccine containing cysteine proteases purified from Porphyromonas gingivalis. Oral Microbiol Immunol 22:162-8.

227. O'Brien-Simpson NM, Pathirana RD, Paolini RA, Chen YY, Veith PD, Tam V, Ally N, Pike RN, Reynolds EC. 2005. An immune response directed to proteinase and adhesin functional epitopes protects against Porphyromonas gingivalis-induced periodontal bone loss. J Immunol 175:3980-9.

228. Cowburn AS, Condliffe AM, Farahi N, Summers C, Chilvers ER. 2008. Advances in neutrophil biology: clinical implications. Chest 134:606-612.

229. Burgos RA, Hidalgo MA, Figueroa CD, Conejeros I, Hancke JL. 2009. New potential targets to modulate neutrophil function in inflammation. Mini Rev Med Chem 9:153-68.

230. Hajishengallis G, Lamont RJ. 2014. Breaking bad: Manipulation of the host response by Porphyromonas gingivalis. European Journal of Immunology 44:328-338.

231. Eke PI, Dye BA, Wei L, Slade GD, Thornton-Evans GO, Borgnakke WS, Taylor GW, Page RC, Beck JD, Genco RJ. 2015. Update on Prevalence of Periodontitis in Adults in the United States: NHANES 2009 to 2012. Journal of Periodontology 86:611-622.

232. Maddi A, Scannapieco FA. 2013. Oral biofilms, oral and periodontal infections, and systemic disease. Am J Dent 26:249-54.

233. Kumar PS. 2013. Oral microbiota and systemic disease. Anaerobe 24:90-93.

234. Kumar PS, Griffen AL, Moeschberger ML, Leys EJ. 2005. Identification of candidate periodontal pathogens and beneficial species by quantitative $16 \mathrm{~S}$ clonal analysis. J Clin Microbiol 43:3944-55.

235. Hajishengallis G, Lamont RJ. Dancing with the Stars: How Choreographed Bacterial Interactions Dictate Nososymbiocity and Give Rise to Keystone Pathogens, Accessory Pathogens, and Pathobionts. Trends in Microbiology doi:http://dx.doi.org/10.1016/j.tim.2016.02.010.

236. Hajishengallis G. 2014. Immunomicrobial pathogenesis of periodontitis: keystones, pathobionts, and host response. Trends in Immunology 35:3-11.

237. Abusleme L, Dupuy AK, Dutzan N, Silva N, Burleson JA, Strausbaugh LD, Gamonal J, Diaz PI. 2013. The subgingival microbiome in health and periodontitis and its relationship with community biomass and inflammation. ISME J 7:1016-1025.

238. Oliveira RRDS, Fermiano D, Feres M, Figueiredo LC, Teles FRF, Soares GMS, Faveri M. 2016. Levels of Candidate Periodontal Pathogens in Subgingival Biofilm. Journal of Dental Research 95:711-718. 
239. Chen H, Liu Y, Zhang M, Wang G, Qi Z, Bridgewater L, Zhao L, Tang Z, Pang X. 2015. A Filifactor alocis-centered co-occurrence group associates with periodontitis across different oral habitats. Scientific Reports 5:9053.

240. Sima C, Gastfreund S, Sun C, Glogauer M. 2014. Rac-Null Leukocytes Are Associated with Increased Inflammation-Mediated Alveolar Bone Loss. The American Journal of Pathology 184:472-482.

241. Hajishengallis G. 2014. Immunomicrobial pathogenesis of periodontitis: keystones, pathobionts, and host response. Trends Immunol 35:3-11.

242. Nussbaum G, Shapira L. 2011. How has neutrophil research improved our understanding of periodontal pathogenesis? Journal of Clinical Periodontology 38:49-59.

243. Roberts HM, Ling MR, Insall R, Kalna G, Spengler J, Grant MM, Chapple ILC. 2015. Impaired neutrophil directional chemotactic accuracy in chronic periodontitis patients. Journal of Clinical Periodontology 42:1-11.

244. Pellmé S, Mörgelin M, Tapper H, Mellqvist U-H, Dahlgren C, Karlsson A. 2006. Localization of human neutrophil interleukin-8 (CXCL-8) to organelle(s) distinct from the classical granules and secretory vesicles. Journal of Leukocyte Biology 79:564-573.

245. Bazzoni F, Cassatella MA, Rossi F, Ceska M, Dewald B, Baggiolini M. 1991. Phagocytosing neutrophils produce and release high amounts of the neutrophil-activating peptide 1/interleukin 8. The Journal of Experimental Medicine 173:771-774.

246. Hayashi F, Means TK, Luster AD. 2003. Toll-like receptors stimulate human neutrophil function. Blood 102:2660-2669.

247. Aomatsu K, Kato T, Fujita H, Hato F, Oshitani N, Kamata N, Tamura T, Arakawa T, Kitagawa S. 2008. Toll-like receptor agonists stimulate human neutrophil migration via activation of mitogen-activated protein kinases. Immunology 123:171-180.

248. Ward RA, Nakamura M, McLeish KR. 2000. Priming of the Neutrophil Respiratory Burst Involves p38 Mitogen-activated Protein Kinase-dependent Exocytosis of Flavocytochrome b 558-containing Granules. Journal of Biological Chemistry 275:36713-36719.

249. Coxon PY, Rane MJ, Uriarte S, Powell DW, Singh S, Butt W, Chen Q, McLeish KR. 2003. MAPK-activated protein kinase-2 participates in p38 MAPK-dependent and ERKdependent functions in human neutrophils. Cell Signal 15:993-1001.

250. Soehnlein O. 2009. An elegant defense: how neutrophils shape the immune response. Trends Immunol 30:511-2.

251. Becker EL, Showell HJ, Henson PM, Hsu LS. 1974. The ability of chemotactic factors to induce lysosomal enzyme release. I. The characteristics of the release, the importance of surfaces and the relation of enzyme release to chemotactic responsiveness. J Immunol 112:2047-54.

252. Colvin RA, Means TK, Diefenbach TJ, Moita LF, Friday RP, Sever S, Campanella GS, Abrazinski T, Manice LA, Moita C, Andrews NW, Wu D, Hacohen N, Luster AD. 2010. Synaptotagmin-mediated vesicle fusion regulates cell migration. Nat Immunol 11:495502 .

253. Glushkova OV, Parfenyuk SB, Khrenov MO, Novoselova TV, Lunin SM, Fesenko EE, Novoselova EG. 2013. Inhibitors of TLR-4, NF- $\kappa$ B, and SAPK/JNK signaling reduce the toxic effect of lipopolysaccharide on RAW 264.7 cells. Journal of Immunotoxicology 10:133-140.

254. Hajishengallis E, Hajishengallis G. 2014. Neutrophil Homeostasis and Periodontal Health in Children and Adults. Journal of Dental Research 93:231-237.

255. Hajishengallis G, Moutsopoulos NM, Hajishengallis E, Chavakis T. Immune and regulatory functions of neutrophils in inflammatory bone loss. Seminars in Immunology doi:http://dx.doi.org/10.1016/j.smim.2016.02.002. 
256. Foxman EF, Campbell JJ, Butcher EC. 1997. Multistep Navigation and the Combinatorial Control of Leukocyte Chemotaxis. The Journal of Cell Biology 139:13491360.

257. Fan J, Malik AB. 2003. Toll-like receptor-4 (TLR4) signaling augments chemokineinduced neutrophil migration by modulating cell surface expression of chemokine receptors. Nat Med 9:315-321.

258. Kim D, Haynes CL. 2013. The role of p38 MAPK in neutrophil functions: single cell chemotaxis and surface marker expression. Analyst 138:6826-33.

259. Zu YL, Qi J, Gilchrist A, Fernandez GA, Vazquez-Abad D, Kreutzer DL, Huang CK, Sha'afi RI. 1998. p38 mitogen-activated protein kinase activation is required for human neutrophil function triggered by TNF-alpha or FMLP stimulation. J Immunol 160:19829.

260. Knall C, Worthen GS, Johnson GL. 1997. Interleukin 8-stimulated phosphatidylinositol3-kinase activity regulates the migration of human neutrophils independent of extracellular signal-regulated kinase and p38 mitogen-activated protein kinases. Proceedings of the National Academy of Sciences 94:3052-3057.

261. Liu X, Ma B, Malik AB, Tang H, Yang T, Sun B, Wang G, Minshall RD, Li Y, Zhao Y, Ye RD, Xu J. 2012. Bidirectional regulation of neutrophil migration by mitogen-activated protein kinases. Nat Immunol 13:457-464.

262. Rorvig S, Ostergaard O, Heegaard NH, Borregaard N. 2013. Proteome profiling of human neutrophil granule subsets, secretory vesicles, and cell membrane: correlation with transcriptome profiling of neutrophil precursors. J Leukoc Biol 94:711-21.

263. Catz SD. 2014. The role of Rab27a in the regulation of neutrophil function. Cell Microbiol doi:10.1111/cmi.12328.

264. Dib K, Perecko T, Jenei V, McFarlane C, Comer D, Brown V, Katebe M, Scheithauer T, Thurmond RL, Chazot PL, Ennis M. 2014. The histamine H4 receptor is a potent inhibitor of adhesion-dependent degranulation in human neutrophils. J Leukoc Biol doi:10.1189/jlb.2AB0813-432RR.

265. Kim D, Haynes CL. 2013. The role of p38 MAPK in neutrophil functions: single cell chemotaxis and surface marker expression. Analyst 138:6826-6833.

266. Visser MB, Sun C-X, Koh A, Ellen RP, Glogauer M. 2013. <italic>Treponema denticola $<$ italic $>$ Major Outer Sheath Protein Impairs the Cellular Phosphoinositide Balance That Regulates Neutrophil Chemotaxis. PLoS ONE 8:e66209.

267. Takeuchi H, Hirano T, Whitmore SE, Morisaki I, Amano A, Lamont RJ. 2013. The serine phosphatase SerB of Porphyromonas gingivalis suppresses IL-8 production by dephosphorylation of NF-kappaB RelA/p65. PLoS Pathog 9:e1003326.

268. Zenobia C, Hajishengallis G. 2015. Porphyromonas gingivalis virulence factors involved in subversion of leukocytes and microbial dysbiosis. Virulence 6:236-243.

269. Uriarte SM, Jog NR, Luerman GC, Bhimani S, Ward RA, McLeish KR. 2009. Counterregulation of clathrin-mediated endocytosis by the actin and microtubular cytoskeleton in human neutrophils. Am J Physiol Cell Physiol 296:C857-67.

270. Nauseef WM. 2007. How human neutrophils kill and degrade microbes: an integrated view. Immunol Rev 219:88-102.

271. Bodey GP, Buckley M, Sathe YS, Freireich EJ. 1966. Quantitative relationships between circulating leukocytes and infection in patients with acute leukemia. Ann Intern Med 64:328-40.

272. Malech HL, Nauseef WM. 1997. Primary inherited defects in neutrophil function: etiology and treatment. Semin Hematol 34:279-90.

273. Aderem A, Underhill DM. 1999. Mechanisms of phagocytosis in macrophages. Annu Rev Immunol 17:593-623. 
274. Underhill DM, Ozinsky A. 2002. Phagocytosis of microbes: complexity in action. Annu Rev Immunol 20:825-52.

275. Thomas EL, Lehrer RI, Rest RF. 1988. Human neutrophil antimicrobial activity. Rev Infect Dis 10 Suppl 2:S450-6.

276. DeLeo FR, Quinn MT. 1996. Assembly of the phagocyte NADPH oxidase: molecular interaction of oxidase proteins. J Leukoc Biol 60:677-91.

277. Dinauer MC. 1993. The respiratory burst oxidase and the molecular genetics of chronic granulomatous disease. Crit Rev Clin Lab Sci 30:329-69.

278. Thrasher AJ, Keep NH, Wientjes F, Segal AW. 1994. Chronic granulomatous disease. Biochim Biophys Acta 1227:1-24.

279. Roos D, de Boer M, Kuribayashi F, Meischl C, Weening RS, Segal AW, Ahlin A, Nemet K, Hossle JP, Bernatowska-Matuszkiewicz E, Middleton-Price H. 1996. Mutations in the $\mathrm{X}$-linked and autosomal recessive forms of chronic granulomatous disease. Blood 87:1663-81.

280. Cross AR, Curnutte JT, Rae J, Heyworth PG. 1996. Hematologically important mutations: X-linked chronic granulomatous disease. Blood Cells Mol Dis 22:90-5.

281. Nauseef WM. 2014. Detection of superoxide anion and hydrogen peroxide production by cellular NADPH oxidases. Biochim Biophys Acta 1840:757-67.

282. Sheppard FR, Kelher MR, Moore EE, McLaughlin NJ, Banerjee A, Silliman CC. 2005. Structural organization of the neutrophil NADPH oxidase: phosphorylation and translocation during priming and activation. J Leukoc Biol 78:1025-42.

283. DeChatelet LR, Shirley PS, Johnston RB, Jr. 1976. Effect of phorbol myristate acetate on the oxidative metabolism of human polymorphonuclear leukocytes. Blood 47:545-54.

284. Whitmore LC, Hook JS, Philiph AR, Hilkin BM, Bing X, Ahn C, Wong HR, Ferguson PJ, Moreland JG. 2016. A Common Genetic Variant in TLR1 Enhances Human Neutrophil Priming and Impacts Length of Intensive Care Stay in Pediatric Sepsis. J Immunol 196:1376-86.

285. DeLeo FR, Renee J, McCormick S, Nakamura M, Apicella M, Weiss JP, Nauseef WM. 1998. Neutrophils exposed to bacterial lipopolysaccharide upregulate NADPH oxidase assembly. J Clin Invest 101:455-63.

286. Kanzaki H, Wada S, Narimiya T, Yamaguchi Y, Katsumata Y, Itohiya K, Fukaya S, Miyamoto Y, Nakamura Y. 2017. Pathways that Regulate ROS Scavenging Enzymes, and Their Role in Defense Against Tissue Destruction in Periodontitis. Front Physiol $8: 351$.

287. Matthews JB, Wright HJ, Roberts A, Cooper PR, Chapple IL. 2007. Hyperactivity and reactivity of peripheral blood neutrophils in chronic periodontitis. Clin Exp Immunol 147:255-64.

288. Canakci CF, Cicek Y, Canakci V. 2005. Reactive oxygen species and human inflammatory periodontal diseases. Biochemistry (Mosc) 70:619-28.

289. Miricescu D, Totan A, Calenic B, Mocanu B, Didilescu A, Mohora M, Spinu T, Greabu M. 2014. Salivary biomarkers: relationship between oxidative stress and alveolar bone loss in chronic periodontitis. Acta Odontol Scand 72:42-7.

290. Esterbauer H, Schaur RJ, Zollner H. 1991. Chemistry and biochemistry of 4hydroxynonenal, malonaldehyde and related aldehydes. Free Radic Biol Med 11:81-128.

291. Wells PG, McCallum GP, Chen CS, Henderson JT, Lee CJ, Perstin J, Preston TJ, Wiley MJ, Wong AW. 2009. Oxidative stress in developmental origins of disease: teratogenesis, neurodevelopmental deficits, and cancer. Toxicol Sci 108:4-18.

292. Chang MC, Tsai YL, Chen YW, Chan CP, Huang CF, Lan WC, Lin CC, Lan WH, Jeng JH. 2013. Butyrate induces reactive oxygen species production and affects cell cycle progression in human gingival fibroblasts. J Periodontal Res 48:66-73. 
293. Yu JY, Lee SY, Son YO, Shi X, Park SS, Lee JC. 2012. Continuous presence of $\mathrm{H}(2) \mathrm{O}(2)$ induces mitochondrial-mediated, MAPK- and caspase-independent growth inhibition and cytotoxicity in human gingival fibroblasts. Toxicol In Vitro 26:561-70.

294. Ha H, Kwak HB, Lee SW, Jin HM, Kim HM, Kim HH, Lee ZH. 2004. Reactive oxygen species mediate RANK signaling in osteoclasts. Exp Cell Res 301:119-27.

295. Fuchs B, Schiller J. 2014. Glycosaminoglycan degradation by selected reactive oxygen species. Antioxid Redox Signal 21:1044-62.

296. Dasgupta J, Kar S, Van Remmen H, Melendez JA. 2009. Age-dependent increases in interstitial collagenase and MAP Kinase levels are exacerbated by superoxide dismutase deficiencies. Exp Gerontol 44:503-10.

297. Kar S, Subbaram S, Carrico PM, Melendez JA. 2010. Redox-control of matrix metalloproteinase-1: a critical link between free radicals, matrix remodeling and degenerative disease. Respir Physiol Neurobiol 174:299-306.

298. Sawamoto Y, Sugano N, Tanaka H, Ito K. 2005. Detection of periodontopathic bacteria and an oxidative stress marker in saliva from periodontitis patients. Oral Microbiol Immunol 20:216-20.

299. Almerich-Silla JM, Montiel-Company JM, Pastor S, Serrano F, Puig-Silla M, Dasi F. 2015. Oxidative Stress Parameters in Saliva and Its Association with Periodontal Disease and Types of Bacteria. Dis Markers 2015:653537.

300. Banasova L, Kamodyova N, Jansakova K, Tothova L, Stanko P, Turna J, Celec P. 2015. Salivary DNA and markers of oxidative stress in patients with chronic periodontitis. Clin Oral Investig 19:201-7.

301. Tothova L, Kamodyova N, Cervenka T, Celec P. 2015. Salivary markers of oxidative stress in oral diseases. Front Cell Infect Microbiol 5:73.

302. Ji S, Hyun J, Park E, Lee BL, Kim KK, Choi Y. 2007. Susceptibility of various oral bacteria to antimicrobial peptides and to phagocytosis by neutrophils. J Periodontal Res 42:410-9.

303. Guentsch A, Puklo M, Preshaw PM, Glockmann E, Pfister W, Potempa J, Eick S. 2009. Neutrophils in chronic and aggressive periodontitis in interaction with Porphyromonas gingivalis and Aggregatibacter actinomycetemcomitans. J Periodontal Res 44:368-77.

304. Shin J, Ji S, Choi Y. 2008. Ability of oral bacteria to induce tissue-destructive molecules from human neutrophils. Oral Dis 14:327-34.

305. Yamazaki T, Miyamoto M, Yamada S, Okuda K, Ishihara K. 2006. Surface protease of Treponema denticola hydrolyzes $\mathrm{C} 3$ and influences function of polymorphonuclear leukocytes. Microbes Infect 8:1758-63.

306. Jayaprakash K, Demirel I, Khalaf H, Bengtsson T. 2015. The role of phagocytosis, oxidative burst and neutrophil extracellular traps in the interaction between neutrophils and the periodontal pathogen Porphyromonas gingivalis. Mol Oral Microbiol 30:361-75.

307. Jimenez Flores E, Tian S, Sizova M, Epstein SS, Lamont RJ, Uriarte SM. 2017. Peptoanaerobacter stomatis Primes Human Neutrophils and Induces Granule Exocytosis. Infect Immun 85.

308. Gallois A, Klein JR, Allen LA, Jones BD, Nauseef WM. 2001. Salmonella pathogenicity island 2-encoded type III secretion system mediates exclusion of NADPH oxidase assembly from the phagosomal membrane. J Immunol 166:5741-8.

309. Mohapatra NP, Soni S, Rajaram MV, Dang PM, Reilly TJ, El-Benna J, Clay CD, Schlesinger LS, Gunn JS. 2010. Francisella acid phosphatases inactivate the NADPH oxidase in human phagocytes. J Immunol 184:5141-50.

310. Lodge R, Diallo TO, Descoteaux A. 2006. Leishmania donovani lipophosphoglycan blocks NADPH oxidase assembly at the phagosome membrane. Cell Microbiol 8:192231. 
311. Siemsen DW, Kirpotina LN, Jutila MA, Quinn MT. 2009. Inhibition of the human neutrophil NADPH oxidase by Coxiella burnetii. Microbes Infect 11:671-9.

312. Allen LA, Allgood JA, Han X, Wittine LM. 2005. Phosphoinositide3-kinase regulates actin polymerization during delayed phagocytosis of Helicobacter pylori. J Leukoc Biol 78:220-30.

313. Carlyon JA, Fikrig E. 2006. Mechanisms of evasion of neutrophil killing by Anaplasma phagocytophilum. Curr Opin Hematol 13:28-33.

314. Nunes P, Demaurex N, Dinauer MC. 2013. Regulation of the NADPH oxidase and associated ion fluxes during phagocytosis. Traffic 14:1118-31.

315. Carlyon JA, Abdel-Latif D, Pypaert M, Lacy P, Fikrig E. 2004. Anaplasma phagocytophilum utilizes multiple host evasion mechanisms to thwart NADPH oxidasemediated killing during neutrophil infection. Infect Immun 72:4772-83.

316. Lam GY, Fattouh R, Muise AM, Grinstein S, Higgins DE, Brumell JH. 2011. Listeriolysin $\mathrm{O}$ suppresses phospholipase $\mathrm{C}$-mediated activation of the microbicidal NADPH oxidase to promote Listeria monocytogenes infection. Cell Host Microbe 10:627-34.

317. DeLeo FR, Allen LA, Apicella M, Nauseef WM. 1999. NADPH oxidase activation and assembly during phagocytosis. J Immunol 163:6732-40.

318. Jann NJ, Schmaler M, Ferracin F, Landmann R. 2011. TLR2 enhances NADPH oxidase activity and killing of Staphylococcus aureus by PMN. Immunol Lett 135:17-23.

319. Johnson MB, Criss AK. 2013. Neisseria gonorrhoeae phagosomes delay fusion with primary granules to enhance bacterial survival inside human neutrophils. Cell Microbiol 15:1323-40.

320. Keith KE, Hynes DW, Sholdice JE, Valvano MA. 2009. Delayed association of the NADPH oxidase complex with macrophage vacuoles containing the opportunistic pathogen Burkholderia cenocepacia. Microbiology 155:1004-15.

321. Laroux FS, Romero X, Wetzler L, Engel P, Terhorst C. 2005. Cutting edge: MyD88 controls phagocyte NADPH oxidase function and killing of gram-negative bacteria. $\mathrm{J}$ Immunol 175:5596-600.

322. Rosales-Reyes R, Skeldon AM, Aubert DF, Valvano MA. 2012. The Type VI secretion system of Burkholderia cenocepacia affects multiple Rho family GTPases disrupting the actin cytoskeleton and the assembly of NADPH oxidase complex in macrophages. Cell Microbiol 14:255-73.

323. Serezani CH, Chung J, Ballinger MN, Moore BB, Aronoff DM, Peters-Golden M. 2007. Prostaglandin E2 suppresses bacterial killing in alveolar macrophages by inhibiting NADPH oxidase. Am J Respir Cell Mol Biol 37:562-70.

324. Li XJ, Tian W, Stull ND, Grinstein S, Atkinson S, Dinauer MC. 2009. A fluorescently tagged $\mathrm{C}$-terminal fragment of $\mathrm{p} 47 \mathrm{phox}$ detects NADPH oxidase dynamics during phagocytosis. Mol Biol Cell 20:1520-32.

325. Armstrong CL, Miralda I, Neff AC, Tian S, Vashishta A, Perez L, Le J, Lamont RJ, Uriarte SM. 2016. Filifactor alocis Promotes Neutrophil Degranulation and Chemotactic Activity. Infect Immun 84:3423-3433.

326. Dupre-Crochet S, Erard M, Nubetae O. 2013. ROS production in phagocytes: why, when, and where? J Leukoc Biol 94:657-70.

327. Rigby KM, DeLeo FR. 2012. Neutrophils in innate host defense against Staphylococcus aureus infections. Semin Immunopathol 34:237-59.

328. Beavers WN, Skaar EP. 2016. Neutrophil-generated oxidative stress and protein damage in Staphylococcus aureus. Pathog Dis 74.

329. Spaan AN, Surewaard BG, Nijland R, van Strijp JA. 2013. Neutrophils versus Staphylococcus aureus: a biological tug of war. Annu Rev Microbiol 67:629-50. 
330. Katsuragi H, Ohtake M, Kurasawa I, Saito K. 2003. Intracellular production and extracellular release of oxygen radicals by PMNs and oxidative stress on PMNs during phagocytosis of periodontopathic bacteria. Odontology 91:13-8.

331. Hajishengallis G, Lamont RJ. 2012. Beyond the red complex and into more complexity: the polymicrobial synergy and dysbiosis (PSD) model of periodontal disease etiology.

Mol Oral Microbiol 27:409-19.

332. Allen LA. 2003. Mechanisms of pathogenesis: evasion of killing by polymorphonuclear leukocytes. Microbes Infect 5:1329-35.

333. Criss AK, Katz BZ, Seifert HS. 2009. Resistance of Neisseria gonorrhoeae to nonoxidative killing by adherent human polymorphonuclear leucocytes. Cell Microbiol 11:1074-87.

334. Johnson MB, Ball LM, Daily KP, Martin JN, Columbus L, Criss AK. 2015. Opa+ Neisseria gonorrhoeae exhibits reduced survival in human neutrophils via Src family kinase-mediated bacterial trafficking into mature phagolysosomes. Cell Microbiol 17:648-65.

335. Droge W. 2002. Free radicals in the physiological control of cell function. Physiol Rev $82: 47-95$.

336. Guerra FE, Addison CB, de Jong NW, Azzolino J, Pallister KB, van Strijp JA, Voyich JM. 2016. Staphylococcus aureus SaeR/S-regulated factors reduce human neutrophil reactive oxygen species production. J Leukoc Biol 100:1005-1010.

337. Horsburgh MJ, Clements MO, Crossley H, Ingham E, Foster SJ. 2001. PerR controls oxidative stress resistance and iron storage proteins and is required for virulence in Staphylococcus aureus. Infect Immun 69:3744-54.

338. Karavolos MH, Horsburgh MJ, Ingham E, Foster SJ. 2003. Role and regulation of the superoxide dismutases of Staphylococcus aureus. Microbiology 149:2749-58.

339. Liu GY, Essex A, Buchanan JT, Datta V, Hoffman HM, Bastian JF, Fierer J, Nizet V. 2005. Staphylococcus aureus golden pigment impairs neutrophil killing and promotes virulence through its antioxidant activity. J Exp Med 202:209-15.

340. Genestet C, Le Gouellec A, Chaker H, Polack B, Guery B, Toussaint B, Stasia MJ. 2014. Scavenging of reactive oxygen species by tryptophan metabolites helps Pseudomonas aeruginosa escape neutrophil killing. Free Radic Biol Med 73:400-10.

341. Saha P, Yeoh BS, Olvera RA, Xiao X, Singh V, Awasthi D, Subramanian BC, Chen Q, Dikshit M, Wang Y, Parent CA, Vijay-Kumar M. 2017. Bacterial Siderophores Hijack Neutrophil Functions. J Immunol 198:4293-4303.

342. Murakawa H, Bland CE, Willis WT, Dallman PR. 1987. Iron deficiency and neutrophil function: different rates of correction of the depressions in oxidative burst and myeloperoxidase activity after iron treatment. Blood 69:1464-8.

343. Nemeth E, Rivera S, Gabayan V, Keller C, Taudorf S, Pedersen BK, Ganz T. 2004. IL-6 mediates hypoferremia of inflammation by inducing the synthesis of the iron regulatory hormone hepcidin. J Clin Invest 113:1271-6.

344. Newton GL, Rawat M, La Clair JJ, Jothivasan VK, Budiarto T, Hamilton CJ, Claiborne A, Helmann JD, Fahey RC. 2009. Bacillithiol is an antioxidant thiol produced in Bacilli. Nat Chem Biol 5:625-7.

345. Upton H, Newton GL, Gushiken M, Lo K, Holden D, Fahey RC, Rawat M. 2012. Characterization of BshA, bacillithiol glycosyltransferase from Staphylococcus aureus and Bacillus subtilis. FEBS Lett 586:1004-8.

346. Posada AC, Kolar SL, Dusi RG, Francois P, Roberts AA, Hamilton CJ, Liu GY, Cheung A. 2014. Importance of bacillithiol in the oxidative stress response of Staphylococcus aureus. Infect Immun 82:316-32.

347. Allen LA. 2006. Interview with Dr. Lee-Ann Allen regarding Pivotal Advance: Francisella tularensis LVS evades killing by human neutrophils via inhibition of the 
respiratory burst and phagosome escape. Interview by Helene F. Rosenberg. J Leukoc Biol 80:1222-3.

348. Babior BM. 1984. Oxidants from phagocytes: agents of defense and destruction. Blood 64:959-66.

349. Babior BM. 2000. Phagocytes and oxidative stress. Am J Med 109:33-44.

350. El-Benna J, Dang PM, Gougerot-Pocidalo MA. 2008. Priming of the neutrophil NADPH oxidase activation: role of $\mathrm{p} 47$ phox phosphorylation and NOX2 mobilization to the plasma membrane. Semin Immunopathol 30:279-89.

351. Karimi G, Houee Levin C, Dagher MC, Baciou L, Bizouarn T. 2014. Assembly of phagocyte NADPH oxidase: A concerted binding process? Biochim Biophys Acta 1840:3277-83.

352. Dusi S, Della Bianca V, Grzeskowiak M, Rossi F. 1993. Relationship between phosphorylation and translocation to the plasma membrane of $\mathrm{p} 47 \mathrm{phox}$ and $\mathrm{p} 67 \mathrm{phox}$ and activation of the NADPH oxidase in normal and $\mathrm{Ca}(2+)$-depleted human neutrophils. Biochem J 290 ( Pt 1):173-8.

353. Heyworth PG, Badwey JA. 1990. Protein phosphorylation associated with the stimulation of neutrophils. Modulation of superoxide production by protein kinase $\mathrm{C}$ and calcium. $\mathrm{J}$ Bioenerg Biomembr 22:1-26.

354. Miyahara M, Okimasu E, Uchida H, Eisuke, Sato F, Yamamoto M, Utsumi K. 1988. Charge-dependent regulation of NADPH oxidase activities in intact and subcellular systems of polymorphonuclear leukocytes. Biochim Biophys Acta 971:46-54.

355. Azuma H, Oomi H, Sasaki K, Kawabata I, Sakaino T, Koyano S, Suzutani T, Nunoi H, Okuno A. 1995. A new mutation in exon 12 of the gp91-phox gene leading to cytochrome b-positive X-linked chronic granulomatous disease. Blood 85:3274-7.

356. Groemping Y, Rittinger K. 2005. Activation and assembly of the NADPH oxidase: a structural perspective. Biochem J 386:401-16.

357. Roberts AW, Kim C, Zhen L, Lowe JB, Kapur R, Petryniak B, Spaetti A, Pollock JD, Borneo JB, Bradford GB, Atkinson SJ, Dinauer MC, Williams DA. 1999. Deficiency of the hematopoietic cell-specific Rho family GTPase Rac2 is characterized by abnormalities in neutrophil function and host defense. Immunity 10:183-96.

358. Yang FC, Kapur R, King AJ, Tao W, Kim C, Borneo J, Breese R, Marshall M, Dinauer MC, Williams DA. 2000. Rac2 stimulates Akt activation affecting BAD/Bcl-XL expression while mediating survival and actin function in primary mast cells. Immunity 12:557-68.

359. Li B, Yu H, Zheng W, Voll R, Na S, Roberts AW, Williams DA, Davis RJ, Ghosh S, Flavell RA. 2000. Role of the guanosine triphosphatase Rac2 in T helper 1 cell differentiation. Science 288:2219-22.

360. Johnson MB, Criss AK. 2013. Fluorescence microscopy methods for determining the viability of bacteria in association with mammalian cells. J Vis Exp doi:10.3791/50729.

361. Cheng OZ, Palaniyar N. 2013. NET balancing: a problem in inflammatory lung diseases. Front Immunol 4:1.

362. Simon D, Simon HU, Yousefi S. 2013. Extracellular DNA traps in allergic, infectious, and autoimmune diseases. Allergy 68:409-16.

363. White PC, Chicca IJ, Cooper PR, Milward MR, Chapple IL. 2016. Neutrophil Extracellular Traps in Periodontitis: A Web of Intrigue. J Dent Res 95:26-34.

364. Fuchs TA, Abed U, Goosmann C, Hurwitz R, Schulze I, Wahn V, Weinrauch Y, Brinkmann V, Zychlinsky A. 2007. Novel cell death program leads to neutrophil extracellular traps. J Cell Biol 176:231-41.

365. Remijsen Q, Kuijpers TW, Wirawan E, Lippens S, Vandenabeele P, Vanden Berghe T. 2011. Dying for a cause: NETosis, mechanisms behind an antimicrobial cell death modality. Cell Death Differ 18:581-8. 
366. Pilsczek FH, Salina D, Poon KK, Fahey C, Yipp BG, Sibley CD, Robbins SM, Green FH, Surette MG, Sugai M, Bowden MG, Hussain M, Zhang K, Kubes P. 2010. A novel mechanism of rapid nuclear neutrophil extracellular trap formation in response to Staphylococcus aureus. J Immunol 185:7413-25.

367. Doke M, Fukamachi H, Morisaki H, Arimoto T, Kataoka H, Kuwata H. 2016. Nucleases from Prevotella intermedia can degrade neutrophil extracellular traps. Mol Oral Microbiol doi:10.1111/omi.12171.

368. Buchanan JT, Simpson AJ, Aziz RK, Liu GY, Kristian SA, Kotb M, Feramisco J, Nizet V. 2006. DNase expression allows the pathogen group A Streptococcus to escape killing in neutrophil extracellular traps. Curr Biol 16:396-400.

369. Sumby P, Barbian KD, Gardner DJ, Whitney AR, Welty DM, Long RD, Bailey JR, Parnell MJ, Hoe NP, Adams GG, Deleo FR, Musser JM. 2005. Extracellular deoxyribonuclease made by group A Streptococcus assists pathogenesis by enhancing evasion of the innate immune response. Proc Natl Acad Sci U S A 102:1679-84.

370. Porschen RK, Sonntag S. 1974. Extracellular deoxyribonuclease production by anaerobic bacteria. Appl Microbiol 27:1031-3.

371. Rudek W, Haque RU. 1976. Extracellular enzymes of the genus Bacteroides. J Clin Microbiol 4:458-60.

372. Dahlen G, Wikstrom M, Moller A. 1983. Production of histolytic enzymes by a combination of oral bacteria with known pathogenicity. J Dent Res 62:1041-4.

373. White P, Sakellari D, Roberts H, Risafi I, Ling M, Cooper P, Milward M, Chapple I. 2016. Peripheral blood neutrophil extracellular trap production and degradation in chronic periodontitis. J Clin Periodontol 43:1041-1049.

374. Brinkmann V, Goosmann C, Kuhn LI, Zychlinsky A. 2012. Automatic quantification of in vitro NET formation. Front Immunol 3:413.

375. Palmer LJ, Damgaard C, Holmstrup P, Nielsen CH. 2016. Influence of complement on neutrophil extracellular trap release induced by bacteria. J Periodontal Res 51:70-6.

376. Metzler KD, Goosmann C, Lubojemska A, Zychlinsky A, Papayannopoulos V. 2014. A myeloperoxidase-containing complex regulates neutrophil elastase release and actin dynamics during NETosis. Cell Rep 8:883-96.

377. Kolaczkowska E, Jenne CN, Surewaard BG, Thanabalasuriar A, Lee WY, Sanz MJ, Mowen K, Opdenakker G, Kubes P. 2015. Molecular mechanisms of NET formation and degradation revealed by intravital imaging in the liver vasculature. Nat Commun 6:6673.

378. Hirschfeld J, Roberts HM, Chapple IL, Parcina M, Jepsen S, Johansson A, Claesson R. 2016. Effects of Aggregatibacter actinomycetemcomitans leukotoxin on neutrophil migration and extracellular trap formation. J Oral Microbiol 8:33070.

379. Riyapa D, Buddhisa S, Korbsrisate S, Cuccui J, Wren BW, Stevens MP, Ato M, Lertmemongkolchai G. 2012. Neutrophil extracellular traps exhibit antibacterial activity against burkholderia pseudomallei and are influenced by bacterial and host factors. Infect Immun 80:3921-9.

380. Hirschfeld J, Dommisch H, Skora P, Horvath G, Latz E, Hoerauf A, Waller T, Kawai T, Jepsen S, Deschner J, Bekeredjian-Ding I. 2015. Neutrophil extracellular trap formation in supragingival biofilms. Int J Med Microbiol 305:453-63.

381. Mohanty T, Sjogren J, Kahn F, Abu-Humaidan AH, Fisker N, Assing K, Morgelin M, Bengtsson AA, Borregaard N, Sorensen OE. 2015. A novel mechanism for NETosis provides antimicrobial defense at the oral mucosa. Blood 126:2128-37.

382. Vitkov L, Klappacher M, Hannig M, Krautgartner WD. 2010. Neutrophil fate in gingival crevicular fluid. Ultrastruct Pathol 34:25-30.

383. Kowanetz M, Wu X, Lee J, Tan M, Hagenbeek T, Qu X, Yu L, Ross J, Korsisaari N, Cao T, Bou-Reslan H, Kallop D, Weimer R, Ludlam MJ, Kaminker JS, Modrusan Z, van Bruggen N, Peale FV, Carano R, Meng YG, Ferrara N. 2010. Granulocyte-colony 
stimulating factor promotes lung metastasis through mobilization of Ly6G+Ly6C+ granulocytes. Proc Natl Acad Sci U S A 107:21248-55.

384. Kaplan MJ, Radic M. 2012. Neutrophil extracellular traps: double-edged swords of innate immunity. J Immunol 189:2689-95.

385. Urban CF, Ermert D, Schmid M, Abu-Abed U, Goosmann C, Nacken W, Brinkmann V, Jungblut PR, Zychlinsky A. 2009. Neutrophil extracellular traps contain calprotectin, a cytosolic protein complex involved in host defense against Candida albicans. PLoS Pathog $5:$ e1000639.

386. Scharrig E, Carestia A, Ferrer MF, Cedola M, Pretre G, Drut R, Picardeau M, Schattner M, Gomez RM. 2015. Neutrophil Extracellular Traps are Involved in the Innate Immune Response to Infection with Leptospira. PLoS Negl Trop Dis 9:e0003927.

387. Bjornsdottir H, Welin A, Michaelsson E, Osla V, Berg S, Christenson K, Sundqvist M, Dahlgren C, Karlsson A, Bylund J. 2015. Neutrophil NET formation is regulated from the inside by myeloperoxidase-processed reactive oxygen species. Free Radic Biol Med 89:1024-35.

388. Malachowa N, Kobayashi SD, Freedman B, Dorward DW, DeLeo FR. 2013. Staphylococcus aureus leukotoxin GH promotes formation of neutrophil extracellular traps. J Immunol 191:6022-9.

389. Arazna M, Pruchniak MP, Demkow U. 2013. Neutrophil extracellular traps in bacterial infections: strategies for escaping from killing. Respir Physiol Neurobiol 187:74-7.

390. Wang L, Shen D, Wu H, Ma Y. 2017. Resistance of hypervirulent Klebsiella pneumoniae to both intracellular and extracellular killing of neutrophils. PLoS One 12:e173638.

391. Wan T, Zhao Y, Fan F, Hu R, Jin X. 2017. Dexamethasone Inhibits S. aureus-Induced Neutrophil Extracellular Pathogen-Killing Mechanism, Possibly through Toll-Like Receptor Regulation. Front Immunol 8:60.

392. Derre-Bobillot A, Cortes-Perez NG, Yamamoto Y, Kharrat P, Couve E, Da Cunha V, Decker P, Boissier MC, Escartin F, Cesselin B, Langella P, Bermudez-Humaran LG, Gaudu P. 2013. Nuclease A (Gbs0661), an extracellular nuclease of Streptococcus agalactiae, attacks the neutrophil extracellular traps and is needed for full virulence. Mol Microbiol 89:518-31.

393. Chang A, Khemlani A, Kang H, Proft T. 2011. Functional analysis of Streptococcus pyogenes nuclease A ( $\mathrm{SpnA}$ ), a novel group A streptococcal virulence factor. Mol Microbiol 79:1629-42.

394. de Buhr N, Neumann A, Jerjomiceva N, von Kockritz-Blickwede M, Baums CG. 2014. Streptococcus suis DNase SsnA contributes to degradation of neutrophil extracellular traps (NETs) and evasion of NET-mediated antimicrobial activity. Microbiology 160:385-95.

395. Morita C, Sumioka R, Nakata M, Okahashi N, Wada S, Yamashiro T, Hayashi M, Hamada S, Sumitomo T, Kawabata S. 2014. Cell wall-anchored nuclease of Streptococcus sanguinis contributes to escape from neutrophil extracellular trap-mediated bacteriocidal activity. PLoS One 9:e103125.

396. Juneau RA, Stevens JS, Apicella MA, Criss AK. 2015. A thermonuclease of Neisseria gonorrhoeae enhances bacterial escape from killing by neutrophil extracellular traps. J Infect Dis 212:316-24.

397. Wartha F, Beiter K, Normark S, Henriques-Normark B. 2007. Neutrophil extracellular traps: casting the NET over pathogenesis. Curr Opin Microbiol 10:52-6.

398. Ma F, Yi L, Yu N, Wang G, Ma Z, Lin H, Fan H. 2017. Streptococcus suis Serotype 2 Biofilms Inhibit the Formation of Neutrophil Extracellular Traps. Front Cell Infect Microbiol 7:86.

399. Bruns S, Kniemeyer O, Hasenberg M, Aimanianda V, Nietzsche S, Thywissen A, Jeron A, Latge JP, Brakhage AA, Gunzer M. 2010. Production of extracellular traps against 
Aspergillus fumigatus in vitro and in infected lung tissue is dependent on invading neutrophils and influenced by hydrophobin RodA. PLoS Pathog 6:e1000873.

400. Ullah I, Ritchie ND, Evans TJ. 2017. The interrelationship between phagocytosis, autophagy and formation of neutrophil extracellular traps following infection of human neutrophils by Streptococcus pneumoniae. Innate Immun doi:10.1177/1753425917704299:1753425917704299.

401. Lim MB, Kuiper JW, Katchky A, Goldberg H, Glogauer M. 2011. Rac2 is required for the formation of neutrophil extracellular traps. J Leukoc Biol 90:771-6.

402. Brinkmann V, Zychlinsky A. 2007. Beneficial suicide: why neutrophils die to make NETs. Nat Rev Microbiol 5:577-82.

403. Demoruelle MK, Harrall KK, Ho L, Purmalek MM, Seto NL, Rothfuss HM, Weisman MH, Solomon JJ, Fischer A, Okamoto Y, Kelmenson LB, Parish MC, Feser M, Fleischer C, Anderson C, Mahler M, Norris JM, Kaplan MJ, Cherrington BD, Holers VM, Deane KD. 2017. Anti-Citrullinated Protein Antibodies Are Associated With Neutrophil Extracellular Traps in the Sputum in Relatives of Rheumatoid Arthritis Patients. Arthritis Rheumatol doi:10.1002/art.40066.

404. Doring Y, Weber C, Soehnlein O. 2013. Footprints of neutrophil extracellular traps as predictors of cardiovascular risk. Arterioscler Thromb Vasc Biol 33:1735-6.

405. Logters T, Margraf S, Altrichter J, Cinatl J, Mitzner S, Windolf J, Scholz M. 2009. The clinical value of neutrophil extracellular traps. Med Microbiol Immunol 198:211-9.

406. Hakkim A, Fuchs TA, Martinez NE, Hess S, Prinz H, Zychlinsky A, Waldmann H. 2011. Activation of the Raf-MEK-ERK pathway is required for neutrophil extracellular trap formation. Nat Chem Biol 7:75-7.

407. Haririan H, Andrukhov O, Bertl K, Lettner S, Kierstein S, Moritz A, Rausch-Fan X. 2014. Microbial analysis of subgingival plaque samples compared to that of whole saliva in patients with periodontitis. J Periodontol 85:819-28.

408. Gorgojo J, Scharrig E, Gomez RM, Harvill ET, Rodriguez ME. 2017. Bordetella parapertussis Circumvents Neutrophil Extracellular Bactericidal Mechanisms. PLoS One 12:e0169936.

409. Vong L, Lorentz RJ, Assa A, Glogauer M, Sherman PM. 2014. Probiotic Lactobacillus rhamnosus inhibits the formation of neutrophil extracellular traps. J Immunol 192:18707.

410. Brinkmann V, Laube B, Abu Abed U, Goosmann C, Zychlinsky A. 2010. Neutrophil extracellular traps: how to generate and visualize them. J Vis Exp doi:10.3791/1724.

411. Kolenbrander PE, Andersen RN, Blehert DS, Egland PG, Foster JS, Palmer RJ, Jr. 2002. Communication among oral bacteria. Microbiol Mol Biol Rev 66:486-505, table of contents.

412. Matute JD, Arias AA, Dinauer MC, Patino PJ. 2005. p40phox: the last NADPH oxidase subunit. Blood Cells Mol Dis 35:291-302.

413. Heit B, Kubes P. 2003. Measuring chemotaxis and chemokinesis: the under-agarose cell migration assay. Sci STKE 2003:P15.

414. Ward RA, Nakamura M, McLeish KR. 2000. Priming of the neutrophil respiratory burst involves p38 mitogen-activated protein kinase-dependent exocytosis of flavocytochrome b558-containing granules. J Biol Chem 275:36713-9.

415. Weiss SJ, Young J, LoBuglio AF, Slivka A, Nimeh NF. 1981. Role of hydrogen peroxide in neutrophil-mediated destruction of cultured endothelial cells. J Clin Invest 68:714-21.

416. Dekker LV, Leitges M, Altschuler G, Mistry N, McDermott A, Roes J, Segal AW. 2000. Protein kinase C-beta contributes to NADPH oxidase activation in neutrophils. Biochem J 347 Pt 1:285-9. 
417. McInturff AM, Cody MJ, Elliott EA, Glenn JW, Rowley JW, Rondina MT, Yost CC. 2012. Mammalian target of rapamycin regulates neutrophil extracellular trap formation via induction of hypoxia-inducible factor 1 alpha. Blood 120:3118-25.

418. Lapponi MJ, Carestia A, Landoni VI, Rivadeneyra L, Etulain J, Negrotto S, Pozner RG, Schattner M. 2013. Regulation of neutrophil extracellular trap formation by antiinflammatory drugs. J Pharmacol Exp Ther 345:430-7. 


\section{CURICULUM VITAE}

\section{Cortney L. Armstrong \\ (321) 525-1858, clarmstrong01@gmail.com}

\section{EDUCATION}

University of Louisville, Louisville, KY

8/12-present

- Pursuing Doctor of Philosophy in Microbiology and Immunology

Expected 8/17

University of Louisville, Louisville, KY

$8 / 12-12 / 14$

- Masters of Science in Microbiology and Immunology

Florida State University, Tallahassee, FL

$5 / 08-4 / 12$

- Bachelor of Science in Biological Sciences, minor in Chemistry and Religion.

\section{RESEARCH EXPERIENCE}

University of Louisville, Louisville, KY

5/13-present

- Ph.D. Researcher

- Studying the interactions between Filifactor alocis, an oral pathogen recently associated with the development of periodontal diseases, and human neutrophils to delineate the mechanisms employed by the bacterium to evade detection and killing by human neutrophils.

Florida State University, Tallahassee, FL

- Undergraduate Student Researcher

- Studied in the field of oceanography sorting, classifying and quantifying deep-sea macrofauna and meiofauna sampled at eight stations from the Eastern Pacific.

\section{TECHNOLOGY TRANSFER EXPERIENCE}

University of Louisville, Louisville, KY

- Intern at the Office of Technology Transfer and Industry Engagement at University of Louisville

- Worked alongside technology transfer managers and the Office of Industry Engagement on performing prior art searches, composing 
marketing abstracts for research disclosures received from University of Louisville researchers, and reaching out to companies whose product development would benefit from the technologies available for licensing at the University of Louisville.

\section{PUBLICATIONS}

Armstrong CL, Miralda I, Neff AC, Tian S, Vashishta A, Perez L, Le J, Lamont RJ, Uriarte SM. 2016. Filifactor alocis promotes neutrophil degranulation and chemotactic activity. Infect Immun 84:3423-3433.

\section{PRESENTATIONS}

Oral presentations:

- University of Louisville's Kidney Disease Program Research Conference, $10 / 15$ Louisville, KY, "Evading the host: a closer look at the interactions between human neutrophils and Filifactor alocis."

- Society for Leukocyte Biology Meeting, Raleigh, NC, "The oral pathogen Filifactor alocis enhances chemotactic functions of human neutrophils"

- University of Louisville's Dental school Student Research Group meeting, Louisville, KY, "Evading the host: a closer look at the interactions between human neutrophils and Filifactor alocis."

Poster presentations:

- Research Louisville, Louisville, KY, "The periodontal pathogen, Filifactor alocis, 10/16 evades human neutrophil oxidative killing without compromising the recruitment of p47phox and p67phox to the bacterial-phagosome."

- Midwest Microbial Pathogenesis Conference, Urbana-Champaign, IL, "The $9 / 16$ periodontal pathogen, Filifactor alocis, evades human neutrophil oxidative killing without compromising the recruitment of $\mathrm{p} 47$ phox and p67phox to the bacterialphagosome."

- Research Louisville, Louisville, KY, "The oral pathogen Filifactor alocis, enhances chemotactic functions of human neutrophils"

- Society for Leukocyte Biology Meeting, Raleigh, NC, "The oral pathogen Filifactor alocis enhances chemotactic functions of human neutrophils"

- Society for Leukocyte Biology Meeting, Salt Lake City, UT, "Enhanced human 10/14 neutrophil activation upon challenge with Filifactor alocis, a recently described oral pathogen"

- Research Louisville, Louisville, KY, "Enhanced human neutrophil activation $\quad$ 9/14 upon challenge with Filifactor alocis, a recently described oral pathogen" 
- Society for Leukocyte Biology Meeting, Newport, RI, "In vivo infection of

Filifactor alocis and its effect on neutrophil infiltration and clearance in a mouse chamber model"

- Research Louisville, Louisville, KY, "In vivo infection of Filifactor alocis and its 9/13 effect on neutrophil infiltration and clearance in a mouse chamber model"

- Benthic Ecology Meeting, Mobile, AL, "What is the abundance of deep-sea 3/11 nematodes compared to that of other deep-sea meiofauna?"

\section{AWARDS}

- Society for Leukocyte Biology Travel Award

$8 / 15$

Awards Committee of the Society for Leukocyte Biology

- Society for Leukocyte Biology Travel Award

Awards Committee of the Society for Leukocyte Biology

- Graduate Student Council Travel Award

Graduate Student Council Travel Administrator, University of Louisville

\section{$\underline{\text { ACTIVITIES }}$}

- Internship at the Office of Technology Transfer and Industry

Engagement at University of Louisville

- $\quad$ Society for Leukocyte Biology

- Member 6/13-present

- Junior Member of the Professional Development Committee 7/16-present

- Junior Editor of the iSLB newsletter

- Microbiology and Immunology Student Organization

- Member

8/12-present

- $\quad$ President

$5 / 15-5 / 16$

\section{VOLUNTEER EXPERIENCE}

- Judge at the DuPont Manual Science Fair, Louisville, KY

- Judge at the Louisville Regional Science and Engineering Fair,

Louisville, KY Category: Biomedical and Health Sciences- senior division

- Judge at the DuPont Manual Science Fair, Louisville, KY 
- Judge at the Louisville Regional Science and Engineering Fair,

Louisville, KY Category: Microbiology- junior division 